FINAL REPORT

\title{
The Oxidation of Zircaloy Fuel Cladding in Water-Cooled Nuclear Reactors
}

\author{
Project Grant No. DE-FG03-02SF-22618 \\ Final Report
}

September 15, 2002 - September 14, 2006

\author{
Submitted by: \\ Digby D. Macdonald (PI), Mirna Urquidi-Macdonald (Co-PI), Yingzi Chen (Graduate \\ Assistant), Jiahe Ai (Post Doctoral Scholar), Pilyeon Park (Graduate Assistant), and Han- \\ Sang Kim (Graduate Assistant). \\ Center for Electrochemical Science and Technology \\ Pennsylvania State University \\ 201 Steidle Building \\ University Park, PA 16802
}

Submitted on:

October 30, 2006 


\begin{abstract}
Our work involves the continued development of the theory of passivity and passivity breakdown, in the form of the Point Defect Model, with the emphasis on zirconium and zirconium alloys in reactor coolant environments, the measurement of critically-important parameters, and the development of a code that can be used by reactor operators to actively manage the accumulation of corrosion damage to the fuel cladding in both BWRs and PWRs. In addition, the modified boiling crevice model has been further developed to describe the concentration of solutes in porous deposits (CRUD) on fuel under boiling (BWRs) and nucleate boiling (PWRs) conditions, in order that we can accurately describe the environment that is contact with the Zircaloy cladding.

In the current report, we have derived the total steady-state current density and the partial anodic and cathodic current densities to establish a deterministic basis for describing the oxidation. The models are "deterministic" because the relevant natural laws are satisfied explicitly, most importantly the conversation of mass and charge and the equivalence of mass and charge (Faraday's law). Cathodic reactions (oxygen reduction and hydrogen evolution) are also included in the models, because there is some evidence that they control the rate of the overall film formation process. Under open circuit conditions, the cathodic reactions, which must occur at the same rate as the zirconium oxidation reaction, are instrumental in determining the corrosion potential and hence the thickness of the barrier and outer layers of the passive film.

Controlled hydrodynamic methods have been used to measure important parameters in the Point Defect Model, which is now being used to describe the growth and breakdown of the passive film on zirconium and on Zircaloy fuel sheathing alloy in water-cooled nuclear reactors. Firstly, polarization studies have been carried to determine the relevant potential ranges for BWR and PWR coolant systems. Impedance and Mott-Schottky analyses have been carried out on steady state passive films at different formation potentials in $0.1 \mathrm{M} \mathrm{B}(\mathrm{OH})_{3}+0.001 \mathrm{M} \mathrm{LiOH}$ solution of $\mathrm{pH}=6.94$ at $250{ }^{\circ} \mathrm{C}$. The pressure was 62 bar $(900 \mathrm{psi})$ to maintain the electrolyte in the liquid state. The relationships between current density and film thickness and the film formation potential satisfy the diagnostic criteria of the PDM for an n-type passive film. The kinetic parameters are being extracted by optimization of the PDM on the experimental impedance data, which are validated as conforming to the constraints of linear system theory by using the Kramers-Kronig (K-K) transforms. Mott-Schottky analysis has shown that the passive film is n-type in electronic character corresponding to a preponderance of oxygen/hydrogen vacancies or metal interstitials in the barrier layer. The donor density, $\mathrm{N}_{\mathrm{D}}$, calculated from the slope of Mott-Schottky plots is in the range of $10^{17} \sim 10^{18} \mathrm{~cm}^{-3}$, which decreases with increasing formation potential, demonstrating that the film is only lowly-doped with electron donors. However, the low doping level probably reflects a composite value for a highly doped, but thin, barrier layer and a thick, stoichiometric outer layer.
\end{abstract}


Besides obtaining the parameters for the Point Defect Model (PDM) from the impedance analysis under steady state conditions, transient analysis is being carried out to yield the desired parameter values as well. The film thickness is being measured using high frequency capacitance methods, as the thickness changes over a voltage cycle. The current is measured simultaneously. The current density transients and the changes of film thickness of zirconium passive film in $0.1 \mathrm{M} \mathrm{B}(\mathrm{OH})_{3}+0.001 \mathrm{M} \mathrm{LiOH}$ solution at $250{ }^{\circ} \mathrm{C}$ and 62 bar have confirmed that the rate law predicted by the PDM can be used to account for zirconium passive film growth and thinning. When stepping the potential in the positive direction, the part of the additional potential drop at the metal/film $(\mathrm{m} / \mathrm{f})$ interface enhances the rate of production of oxygen vacancies, thereby leading to enhanced growth of the film into the metal. Experiment results show that the current density increases very rapidly, and then relaxes to a value that is comparable to that before the potential step was applied. The film thickening process is also very fast. Upon stepping the potential in the negative direction, the film thickness initially decreases at a constant rate, due to the film growth reaction (production of oxygen vacancies) at the metal/film interface being "shut down" by the lack of a sufficient potential drop and film thinning occurs only via dissolution at the film/solution interface. However, as the film thins, the potential drop across the film decreases, due to the constant field strength, and the additional potential drop appears across the metal/film interface. At some point, the film growth reaction at the metal/film interface starts up and the rate increases as the barrier layer continues to thin until it matches the rate of dissolution. At that point, the film has attained a new steady state with a film thickness that is smaller than that prior to imposition of the negative voltage step. The dissolution rate of the passive film formed in the hydrogenated solution is lower than that formed in the solution without hydrogen. An electron charge transfer phenomenon was observed when changing the hydrodynamic conditions of the solution in contact with the sample. The current density measured in the hydrogenated electrolyte increases with increasing rotation rate of the impeller, which indicates that the hydrogen oxidation reaction may occur in parallel with the oxidation of zirconium. The rate law may then be optimized on both the impedance data and the transient data to yield two sets of PDM parameter values that can be compared. Comparison of the parameter values derived from the impedance data at steady state and the thickness and current transient data will provide a powerful means of assessing the validity of the PDM.

In-situ studies have been carried out by using the controlled hydrodynamic apparatus to examine the effects of second phase particles on the electrochemistry of zirconium passive films on different heat treated Zircaloy-4 samples; i.e., as-received, $\beta$ quenched, and $\alpha$-annealed. Heat treatment is used to control the size and density of the second phase particles in the Zircaloy-4 samples and optical microscopy and scanning electron microscopy (SEM) are used to characterize the microstructure and determine the size and density of the second phase particles, respectively. The sizes of these second phase particles are found to be comparable to the thickness of the passive film. The $\beta$ quenched sample was found to have the highest particle density, while the as-received sample has the largest particle size. The appearance of a small spike in the anodic polarization curve is found to be related to the size and density of the second phase particles. The passive film properties are changed at potentials around the spike, as 
confirmed by the measured impedance and capacitance as a function of potential. Second phase particles split the passive region into two parts having different corrosion resistances, except in the $\beta$-quenched as shown by impedance measurements. The $\beta$ quenched Zr-4 sample has the highest corrosion resistance, while the $\alpha$-annealed sample is the lowest. The electronic characters of the passive films formed on the as-received and $\alpha$-annealed zircaloy- 4 samples are much more complex as indicated by the slope changes from positive to negative in the Mott-Schottky plots while positive linear slopes are found for the $\beta$-quenched zircaloy- 4 sample. The donor concentrations are in the range of $10^{17} \sim 10^{18} \mathrm{~cm}^{-3}$, which decreases with increasing formation potential, demonstrating that the film is only lowly-doped with electron donors. However, the low doping level probably reflects a composite value for a highly doped, but thin, barrier layer and a thick, stoichiometric outer layer.

In-situ studies are being carried out to study the electrochemistry of Zircaloy-2 in the hydrogenated high temperature borate buffer solutions. Second phase particles split the passive region into two parts having different corrosion resistances as demonstrated by measuring the impedance as a function of formation potential. Zircaloy-2 shows lower corrosion resistance comparing to Zircaloy-4 in the hydrogenated environments. The passive films formed on the Zircaloy-2 samples are n-type in electronic character as indicated by the positive slopes of Mott-Shcottky plots and the dominant defects in the oxide film over this voltage range are mainly hydrogen vacancies and/or zirconium interstitials. The donor concentrations are in the range of $10^{17} \sim 10^{18} \mathrm{~cm}^{-3}$, which decreases with increasing formation potential, demonstrating that the film is only lowly-doped with electron donors. However, the low doping level probably reflects a composite value for a highly doped, but thin, barrier layer and a thick, stoichiometric outer layer. 


\section{TABLE OF CONTENTS}

Table of Contents $\quad$ i

List of Figures $\quad$ ii

List of Tables vi

I. Introduction 1

II. Objectives and Accomplishments 1

Task 1. Definition of the Interfacial Environment 1

Task 2. PDM Development 8

Task 3. Incorporation of the PDM into the MPM 46

Task 4. Radiochemistry Modeling 49

Task 5. Modeling Growth Stresses 51

Task 6. Experimental Measurement of Model Parameters 51

Task 7. Model Integration $\quad 115$

$\begin{array}{ll}\text { Task 8. Benchmarking and Evaluation } & 115\end{array}$

Task 9. Technology Transfer 116

$\begin{array}{ll}\text { III. Status Summary Of Neri Tasks } & 116\end{array}$

$\begin{array}{ll}\text { IV. Papers and Presentations } & 116\end{array}$

$\begin{array}{lr}\text { V. Student Dissertations } & 118\end{array}$

$\begin{array}{ll}\text { VI. Cost } & 118\end{array}$ 


\section{LIST OF FIGURES}

Figure 1-1. Predicted build up of a concentrated solution in a boiling, 1-cm long crevice with time for a bulk $\mathrm{NaCl}$ concentration of 10-7 $\mathrm{M}(5.8 \mathrm{ppb})$ and a superheat of $28^{\circ} \mathrm{C}$. Note that the concentrated, stationary phase progressively fills the crevice as time increases and ultimately (in the steady state) occupies the entire crevice except for a small region at the crevice mouth [1].

Figure 1-2. Comparison between theory and experiment for the average volume concentration of $\mathrm{Na}^{+}$in a boiling crevice in contact with a bulk solution containing 40 ppm NaOH [1].

Figure 1-3. Volume averaged concentrations as a function of time for a heated crevice with a superheat of $28^{\circ} \mathrm{C}$, a bulk concentration of $\mathrm{NaCl}$ of $10^{-7}$ $\mathrm{M}$, and a bulk system temperature of $280^{\circ} \mathrm{C}$. The iron species are formed by corrosion reactions in the crevice $[1,3]$.

Figure 1-4 Schematic illustration of the modified boiling crevice model

Figure 2-1. Interfacial reactions leading to the generation and annihilation of point defects within the barrier layer and to the formation of both the barrier layer and outer layer of the passive film on zirconium under PWR coolant condition. $Z r=$ zirconium atom, $Z r_{Z r}=$ zirconium cation in a normal cation site on the metal sublattice in the barrier layer, $V_{Z r}^{4^{\prime}}=$ cation vacancy on the metal sublattice in the barrier layer, $\mathrm{Zr}_{i}^{4+}=$ zirconium interstitial in the barrier layer, $V_{H}^{\bullet}=$ hydrogen anion vacancy on the anion sublattice of the barrier layer, $H_{H}=$ hydrogen anion in an anion site on the anion sublattice of the barrier layer.

Figure 2-2. Postulated potential profile in the passive film on zirconium under PWR coolant conditions. Note that the diagram is not to scale and subscript "bl" designates "barrier layer", while "ol" signifies "outer layer"..

Figure 2-3. Interfacial reactions leading to generation and annihilation of point defects within the passive film on zirconium under BWR coolant conditions. $V_{O}^{\bullet \bullet}=$ Oxygen anion vacancy, $O_{O}=$ oxygen ion in a normal site on the oxygen sublattice. The other species are identified in the caption of Figure 2-1.

Figure 2-4. Postulated potential profile in the passive film on zirconium under BWR coolant conditions. Note that the diagram is not to scale and subscript "bl" designates "barrier layer", while "ol" signifies "outer layer".

Figure 2-5. Interfacial reactions leading to the generation and annihilation of point defects within the barrier layer and to the formation of both the barrier 
layer and outer layer of the passive film on zirconium under PWR coolant conditions. $Z r=$ zirconium atom, $Z r_{Z r}=$ zirconium cation in a normal cation site on the metal sublattice in the barrier layer, $V_{H}^{*}=$ hydrogen anion vacancy on the anion sublattice of the barrier layer, $H_{H}$ $=$ hydrogen anion in an anion site on the anion sublattice of the barrier layer. The species are identified in the caption of Fig. 2-1.

Figure 2-6. Equivalent circuit for the total impedance

Figure 2-7. $\quad$ Primary interfacial reactions leading to the generation and annihilation of point defects within the barrier layer and to the formation of both the barrier layer and outer layer of the passive film on zirconium under BWR coolant conditions. The species are identified in the caption of Fig. 2-3.

Figure 2-8. Flow chart of optimization of the Point Defect Model on the experimental electrochemical impedance data.

Figure 2-9. Nyquist and Bode impedance plots for zirconium passive film polarized at each potential for 24 hours for film formation in $0.1 \mathrm{M} \mathrm{B}(\mathrm{OH})_{3}+$ $0.001 \mathrm{M} \mathrm{LiOH}$ solutions with $22 \mathrm{~cm}^{3}(\mathrm{STP}) / \mathrm{kg}\left(\mathrm{H}_{2} \mathrm{O}\right)$ hydrogen at 250 ${ }^{\circ} \mathrm{C}$ and 62 bar and as a function of potential, showing a comparison between the experimental and simulated data.

Figure 2-10. Nyquist and Bode impedance plots for zirconium passive film polarized at each potential for 24 hours for film formation in $0.1 \mathrm{M} \mathrm{B}(\mathrm{OH})_{3}+$ $0.001 \mathrm{M} \mathrm{LiOH}$ solutions at $250{ }^{\circ} \mathrm{C}$ and 62 bar and as a function of potential, showing a comparison between the experimental and simulated data.

Figure 2-11. Comparison between the experimental and simulated data, (a) thickness of outer layer; (b) current density, where the solution is $0.1 \mathrm{M} \mathrm{B}(\mathrm{OH})_{3}+$ $0.001 \mathrm{M} \mathrm{LiOH}$ aqueous solution with $22 \mathrm{~cm}^{3}(\mathrm{STP}) / \mathrm{kg}\left(\mathrm{H}_{2} \mathrm{O}\right)$ hydrogen at $250{ }^{\circ} \mathrm{C}, \mathrm{pH}=6.94$.

Figure 2-12. Comparison between the experimental and simulated data, (a) thickness of outer layer; (b) current density, where the solution is $0.1 \mathrm{M} \mathrm{B}(\mathrm{OH})_{3}+$ $0.001 \mathrm{M} \mathrm{LiOH}$ aqueous solution at $250{ }^{\circ} \mathrm{C}, \mathrm{pH}=6.94$.

Figure 2-13. Comparison of oxygen vacancy diffusivity obtained in this study and those from literatures.

Figure 2-14. Sensitivity analysis, showing the effect of model parameter value change on the fitting curve, where the solution is $0.1 \mathrm{M} \mathrm{B}(\mathrm{OH})_{3}+0.001$ $\mathrm{M} \mathrm{LiOH}$ aqueous solution with $22 \mathrm{~cm}^{3}(\mathrm{STP}) / \mathrm{kg}\left(\mathrm{H}_{2} \mathrm{O}\right)$ hydrogen at 250 ${ }^{\circ} \mathrm{C}, \mathrm{pH}=6.94$. 
Figure 2-15. Sensitivity analysis, showing the effect of model parameter value change on the fitting curve, where the solution is $0.1 \mathrm{M} \mathrm{B}(\mathrm{OH})_{3}+0.001$ $\mathrm{M} \mathrm{LiOH}$ aqueous solution at $250^{\circ} \mathrm{C}, \mathrm{pH}=6.94$.

Figure 2-16. Schematic diagram of phase space analysis. The intersection of (a) and (b) defines the steady state thickness of the meta-stable barrier layer.

Figure 2-17. Linear-linear (a) and $\log$-linear (b) phase space plots for the barrier layer on zirconium in $0.1 \mathrm{M} \mathrm{B}(\mathrm{OH})_{3}+0.001 \mathrm{M} \mathrm{LiOH}$ de-aerated aqueous solution $\left(p H=6.94\right.$ at $250{ }^{\circ} \mathrm{C}$ and $\left.\mathrm{P}=62 \mathrm{bar}\right)$, as a function of applied potential.

Figure 2-18. Phase space plots for the barrier layer of the passive film on zirconium in $0.1 \mathrm{M} \mathrm{B}(\mathrm{OH})_{3}+0.001 \mathrm{M} \mathrm{LiOH}$ de-aerated aqueous solution $\left(\mathrm{T}=250^{\circ} \mathrm{C}\right.$ and $\mathrm{P}=62$ bar) at $0.5 \mathrm{~V}_{\mathrm{SHE}}$, as a function of the $p H$ at the $\mathrm{BOI}$ and the barrier layer thickness.

Figure 2-19. Plot of the steady state thickness of the barrier layer on zirconium in 0.1 $\mathrm{M} \mathrm{B}(\mathrm{OH})_{3}+0.001 \mathrm{M} \mathrm{LiOH}$ de-aerated aqueous solution $\left(\mathrm{T}=250{ }^{\circ} \mathrm{C}\right.$ and $\mathrm{P}=62$ bar) at $0.5 \mathrm{~V}_{\mathrm{SHE}}$, as a function of $\mathrm{pH}$ at the $\mathrm{BOI}$, showing the condition for depassivation $\left(L_{s s} \rightarrow 0\right)$.

Figure 2-20. Plot of the total current density vs $p H$ for passive zirconium in $0.1 \mathrm{M}$ $\mathrm{B}(\mathrm{OH})_{3}+0.001 \mathrm{M} \mathrm{LiOH}$ de-aerated aqueous solution $\left(\mathrm{T}=250{ }^{\circ} \mathrm{C}\right.$ and $\mathrm{P}$ $=62 \mathrm{bar}$ ) at $0.50 \mathrm{~V}_{\text {SHE }} . n$ is assumed to be 0.45 .

Figure 2-21. Corrosion rate vs $p H$ calculated from the total current density as a function of $p H$ for zirconium in $0.1 \mathrm{M} \mathrm{B}(\mathrm{OH})_{3}+0.001 \mathrm{M} \mathrm{LiOH}$ deaerated aqueous solution $\left(\mathrm{T}=250^{\circ} \mathrm{C}\right.$ and $\left.\mathrm{P}=62 \mathrm{bar}\right)$ at a voltage of 0.50 $\mathrm{V}_{\mathrm{SHE}}$. Note that the corrosion rate is predicted to be an exponentially decreasing function of $p H$, and $n$ is assumed to be 0.45 .

Figure 4-1. Variation of ECP around the heat transport circuit of a BWR employing general catalysis (exchange current density multiplier $10^{4}$ ) under NWC $\left(\left[\mathrm{H}_{2}\right] \mathrm{FW}=0.0 \mathrm{ppm}\right)$ and $\mathrm{HWC}\left(\left[\mathrm{H}_{2}\right] \mathrm{FW}=1.0 \mathrm{ppm}\right)$ conditions. Note that the multiplier employed in the calculation is considered to be extreme and probably could not be achieved with deposited noble metal coatings. The value is used for illustrative purposes only.

Figure 5-1. The relationship between the rate of generation of stress and the fluxes of the vacancies across the barrier layer.

Figure 6-1. Picture of the high-temperature/high-pressure controlled hydrodynamic test system used for the electrochemical experiments. The system is designed to operate at temperatures to $300^{\circ} \mathrm{C}$ and at pressures in excess of 100b. However, due to the limitations of PTFE (Poly Tetra Fluoro Ethylene) and the need to carry out long term experiments, the maximum operating temperature is currently restricted to $250^{\circ} \mathrm{C}$. 
Figure 6-2. Schematic of the once-through recirculating flow loop and the controlled hydrodynamic, high-temperature/high-pressure test cell.

Figure 6-3. Potentiodynamic polarization curve for platinized nickel at $300^{\circ} \mathrm{C}$ for $1.4 \mathrm{ppm}$ hydrogen using a voltage step rate of $50 \mathrm{mV} / 20 \mathrm{~s}$ as a function of the rotational velocity of the flow activating impeller. The broken line corresponds to the prediction of the PDM.

Figure 6-4. Comparison of the electrochemical conditions that exist in the cores of BWRs and PWRs in relationship with the equilibrium potentials for the $\mathrm{Zr} / \mathrm{ZrH}_{2}$ and $\mathrm{ZrO}_{2} / \mathrm{Zr}$ reactions. The equilibrium potentials for the PWR core were calculated assuming $[\mathrm{B}]=1000 \mathrm{ppm}$ and $[\mathrm{Li}]=2 \mathrm{ppm}$ while those for the BWR core assumes pure water to be the coolant. The corrosion potentials for the PWR core were taken from UrquidiMacdonald and Macdonald [5] whereas that for the BWR core was taken from Yeh, Macdonald, and Motta [6]. After Park et al [4].

Figure 6-5. A continuous hydride layer and discrete hydride in the Zircaloy-4 cladding tube (average fuel burnup of $67 \mathrm{GWd} / \mathrm{t}$ and fast fluence of 1.3 x $1022 \mathrm{n} / \mathrm{cm}^{2}$ ) [Courtesy of R. Daum, Argone National Laboratory].

Figure 6-6. Comparison of polarization curves for $\mathrm{Zr}$ in the hydrogenated and hydrogen-free environments. The electrolyte was $0.1 \mathrm{M} \mathrm{B}(\mathrm{OH})_{3}+$ $0.001 \mathrm{M} \mathrm{LiOH}$, with the solution reservoir at ambient temperature being sparged with high-purity argon gas or hydrogen gas before and during the experiments. For the hydrogen case, $22 \mathrm{~cm}^{3}(\mathrm{STP}) / \mathrm{kg}\left(\mathrm{H}_{2} \mathrm{O}\right)$ hydrogen was maintained in the reservoir by suitably controlling the total gas pressure in the system. Scan rate $=1.0 \mathrm{mV} / \mathrm{s}, \mathrm{T}=250{ }^{\circ} \mathrm{C}$ and $\mathrm{P}$ $=62$ bar.

Figure 6-7. Dependence of the steady state current density on applied potentials for passive film formed in pure zirconium in $0.1 \mathrm{M} \mathrm{B}(\mathrm{OH})_{3}+0.001 \mathrm{M}$ $\mathrm{LiOH}$ solution with or without hydrogen. The potentials were changed in the negative-to-positive direction. The current data reported here was the value after holding 24 hours at each potential step for film growth. $\mathrm{T}=250{ }^{\circ} \mathrm{C}$ and $\mathrm{P}=62$ bar.

Figure 6-8. Effect of film formation potential on the capacitance of the passive film formed on zirconium in $0.1 \mathrm{M} \mathrm{B}(\mathrm{OH})_{3}+0.001 \mathrm{M} \mathrm{LiOH}$ solution without hydrogen. $\mathrm{T}=250^{\circ} \mathrm{C}$ and $\mathrm{P}=62$ bar.

Figure 6-9. Dependence of reciprocal capacitance for passive zirconium in $0.1 \mathrm{M}$ $\mathrm{B}(\mathrm{OH})_{3}+0.001 \mathrm{M} \mathrm{LiOH}$ solution without hydrogen measured by holding at each potential 24 hours for film growth. $\mathrm{T}=250{ }^{\circ} \mathrm{C}$ and $\mathrm{P}=$ 62 bar.

Figure 6-10. Dependence of the zirconium passive film thickness on applied potential in $0.1 \mathrm{M} \mathrm{B}(\mathrm{OH})_{3}+0.001 \mathrm{M} \mathrm{LiOH}$ solution with 22 $\mathrm{cm}^{3}(\mathrm{STP}) / \mathrm{kg}\left(\mathrm{H}_{2} \mathrm{O}\right)$ or without hydrogen. The potential was changed in the negative-to-positive direction. The film thickness was measured 
after holding potential at each step for 24 hours for film stabilization. $\mathrm{T}$ $=250{ }^{\circ} \mathrm{C}$ and $\mathrm{P}=62$ bar.

Figure 6-11. Comparison of Nyquist (a) and Bode (b) plots for passive zirconium formed at $0.1 \mathrm{~V}_{\text {she }}$ for 24 hours by scanning the frequency in the highto-low direction and immediately from the low-to-high direction in 0.1 $\mathrm{M} \mathrm{B}(\mathrm{OH})_{3}+0.001 \mathrm{M} \mathrm{LiOH}$ solution with $22 \mathrm{~cm}^{3}(\mathrm{STP}) / \mathrm{kg}\left(\mathrm{H}_{2} \mathrm{O}\right)$ hydrogen. $\mathrm{T}=250{ }^{\circ} \mathrm{C}$ and $\mathrm{P}=62$ bar. "High-to-low" means stepping the frequency from high to low direction and "low-to-high" means stepping the frequency in the reverse direction.

Figure 6-12. Typical Kramers-Kronig transforms of the real and imaginary components of the impedance of passive zirconium in $0.1 \mathrm{M} \mathrm{B}(\mathrm{OH})_{3}+$ $0.001 \mathrm{M} \mathrm{LiOH}$ solution at $\mathrm{T}=250^{\circ} \mathrm{C}$ and $\mathrm{P}=62$ bar. The oxide film was formed at $0.6 \mathrm{~V}_{\text {she }}$ "calculated" real and imaginary components were derived from the experimental imaginary and real components, respectively, by using the $\mathrm{K}-\mathrm{K}$ transforms. These data are compared with the experimental real and imaginary components of the impedance.

Figure 6-13. Typical Kramers-Kronig transforms of the real and imaginary components of the impedance of passive zirconium in $0.1 \mathrm{M} \mathrm{B}(\mathrm{OH})_{3}+$ $0.001 \mathrm{M} \mathrm{LiOH}$ solution with $22 \mathrm{~cm}^{3}(\mathrm{STP}) / \mathrm{kg}\left(\mathrm{H}_{2} \mathrm{O}\right)$ hydrogen $(\mathrm{aq})$ at $\mathrm{T}=$ $250^{\circ} \mathrm{C}$ and $\mathrm{P}=62$ bar. The oxide film was formed at a formation potential $0 \mathrm{~V}_{\text {she. }}$ "calculated" real and imaginary components were derived from the experimental imaginary and real components, respectively, by using the $\mathrm{K}-\mathrm{K}$ transforms. These data are compared with the experimental real and imaginary components of the impedance.

Figure 6-14. Impedance spectra for passive zirconium as a function of film formation potential in $0.1 \mathrm{M} \mathrm{B}(\mathrm{OH})_{3}+0.001 \mathrm{M} \mathrm{LiOH}$ solutions with and without added hydrogen. The impedance was measured after holding the potential at each step for 24 hours for film stabilization. The potentials were changed in the negative-to-positive direction. $\mathrm{T}=250^{\circ} \mathrm{C}$ and $\mathrm{P}=$ 62 bar.

Figure 6-15. Time evolution of oxide film impedance of zirconium formed at potential $-0.1 \mathrm{~V}_{\text {she }}$ in $0.1 \mathrm{M} \mathrm{B}(\mathrm{OH})_{3}+0.001 \mathrm{M} \mathrm{LiOH}$ solution with 22 $\mathrm{cm}^{3}(\mathrm{STP}) / \mathrm{kg}\left(\mathrm{H}_{2} \mathrm{O}\right)$ hydrogen. $\mathrm{T}=250{ }^{\circ} \mathrm{C}$ and $\mathrm{P}=62$ bar.

Figure 6-16. Mott-Schottky plots for the $\mathrm{Zr}$ passive film formed at $0.6 \mathrm{~V}_{\text {she }}$ potential in $0.1 \mathrm{M} \mathrm{B}(\mathrm{OH})_{3}+0.001 \mathrm{M} \mathrm{LiOH}$ solution without hydrogen in the reservoir, measured at different scan rates. Sinusoidal excitation frequency $=15 \mathrm{kHz} . \mathrm{T}=250^{\circ} \mathrm{C}$ and $\mathrm{P}=900$ psi. No dependency was found for scan rate $\geq 25 \mathrm{mV} / \mathrm{s}$.

Figure 6-17. Mott-Schottky plots for the $\mathrm{Zr}$ passive film formed at the indicated potentials after stabilization for 24 hours in $0.1 \mathrm{M} \mathrm{B}(\mathrm{OH})_{3}+0.001 \mathrm{M}$ $\mathrm{LiOH}$ solution sparged with argon gas. Voltage sweep rate in the negative direction from the formation voltage $=25 \mathrm{mV} / \mathrm{s} . \mathrm{T}=250{ }^{\circ} \mathrm{C}$ and $\mathrm{P}=62$ bar. 
Figure 6-18. Mott-Schottky plots for passive $\mathrm{Zr}$ at the indicated potentials after stabilization for 24 hours in $0.1 \mathrm{M} \mathrm{B}(\mathrm{OH})_{3}+0.001 \mathrm{M} \mathrm{LiOH}$ solution with $22 \mathrm{~cm}^{3}(\mathrm{STP}) / \mathrm{kg}\left(\mathrm{H}_{2} \mathrm{O}\right)$ hydrogen. Voltage sweep rate in the negative direction from the formation voltage $=25 \mathrm{mV} / \mathrm{s} . \quad \mathrm{T}=250{ }^{\circ} \mathrm{C}$ and $\mathrm{P}=62$ bar.

Figure 6-19. Donor density $N_{D}$ of the passive film formed on $\mathrm{Zr}$ as a function of film formation potential in $0.1 \mathrm{M} \mathrm{B}(\mathrm{OH})_{3}+0.001 \mathrm{M} \mathrm{LiOH}$ solutions with 22 $\mathrm{cm}^{3}(\mathrm{STP}) / \mathrm{kg}\left(\mathrm{H}_{2} \mathrm{O}\right)$ or without hydrogen. $\mathrm{T}=250^{\circ} \mathrm{C}$ and $\mathrm{P}=62$ bar.

Figure 6-20(a). Schematic of the reactions that are postulated to occur at the metal/barrier layer and barrier layer/outer layer interfaces during the formation of the passive film on zirconium under BWR-like conditions according to the Point Defect Model. Note that Reactions (1), (2), (4), (5), and (6) are lattice conservative processes, in that their occurrence does not result in the movement of the interface, whereas Reactions (3) and (7) are non-conservative. $Z r=$ Zirconium atom, $V_{z r}^{4 \prime}=$ cation vacancy on the zirconium sub lattice of the barrier layer, $v_{z r}=$ zirconium vacancy in the metal, $Z_{i}^{4+}=$ cation interstitial in the film, $Z r_{Z r}=$ zirconium cation on the metal sub lattice of the barrier layer, $V_{o}=$ oxygen vacancy on the oxygen sub lattice of the barrier layer, $O_{O}=$ oxygen anion on the oxygen sub lattice of the barrier layer.

Figure 6-20(b). Schematic of the reactions that are postulated to occur at the metal/barrier layer and barrier layer/outer layer interfaces during the formation of the passive film on zirconium under PWR-like conditions according to the Point Defect Model. Note that Reactions (1), (2), (4), (5), and (6) are lattice conservative processes, in that their occurrence does not result in the movement of the interface, whereas Reactions (3), (7), and (8) are non-conservative. $V_{Z r}^{2^{2 n}}=$ cation vacancy on the zirconium sub lattice of the barrier layer, ${ }^{2 r_{i}^{2+}}=$ cation interstitial in the film, $V_{H}=$ hydrogen vacancy on the hydrogen sub lattice of the barrier layer, $H_{H}=$ hydrogen anion on the hydrogen sub lattice of the barrier layer. Note that the destruction of the hydride barrier layer is postulated to occur electrochemically [Reaction (7)] and chemically [Reaction (8)].

Figure 6-21. Experimental transients in current density and film thickness for the growth and thinning of the passive film on zirconium in $0.1 \mathrm{M} \mathrm{B}(\mathrm{OH})_{3}$ and $0.001 \mathrm{M} \mathrm{LiOH}$ solution at $250{ }^{\circ} \mathrm{C}$ and 62 bar under dehydrogenated conditions upon stepping the potential from $0.30 \mathrm{~V}_{\text {she }} \rightarrow 0.90 \mathrm{~V}_{\text {she }} \rightarrow$ $0.60 \mathrm{~V}_{\text {she. }}$ (a) current density, (b) thickness calculated from capacitance $(\mathrm{f}=1 \mathrm{kHz})$.

Figure 6-22. Experimental transients in current density and film thickness for the growth and reduction of the passive film on zirconium in $0.1 \mathrm{M} \mathrm{B}(\mathrm{OH})_{3}$ and $0.001 \mathrm{M} \mathrm{LiOH}$ solution containing $45 \mathrm{~cm}^{3}(\mathrm{STP}) / \mathrm{kg}\left(\mathrm{H}_{2} \mathrm{O}\right)$ hydrogen at $250{ }^{\circ} \mathrm{C}$ and 62 bar upon stepping the potential from $-0.45 \mathrm{~V}_{\text {she }}$ (open 
circuit potential) $\rightarrow 0.48 \mathrm{~V}_{\text {she }} \rightarrow 0.08 \mathrm{~V}_{\text {she. }}$ (a) current density, (b)

thickness calculated from capacitance $(\mathrm{f}=1 \mathrm{kHz})$.

Figure 6-23. The effect of rotation rate on the changes of current density, and film thickness calculated from capacitance measurements of passive film formed at $0.30 \mathrm{~V}_{\text {she }}$ that formed in $0.1 \mathrm{M} \mathrm{B}(\mathrm{OH})_{3}+0.001 \mathrm{M} \mathrm{LiOH}$ solution. $\mathrm{T}=250^{\circ} \mathrm{C}$ and $\mathrm{p}=62 \mathrm{bar}$.

Figure 6-24. The effect of rotation rate on the changes of current density and film thickness calculated from capacitance measurements of passive film formed at $0.38 \mathrm{~V}_{\text {she }}$ in $0.1 \mathrm{M} \mathrm{B}(\mathrm{OH})_{3}+0.001 \mathrm{M} \mathrm{LiOH}$ solution containing 4 ppm hydrogen. $\mathrm{T}=250^{\circ} \mathrm{C}$ and $\mathrm{p}=62 \mathrm{bar}$.

Figure 6-25. Comparison of the current density change with the rotation rate from $100 \mathrm{rpm} \rightarrow 500 \mathrm{rpm} \rightarrow 100 \mathrm{rpm}$ for system with and without hydrogen. The system contains hydrogen shows electron charge transfer phenomenon.

Figure 6-26: Average precipitate diameter versus CAP in Zircaloy-4 [20].

Figure 6-27: As-received $\mathrm{Zr}-4$ sample showing equiaxed $\alpha$ grain structure. Swab etched and anodized at $15 \mathrm{~V}$. Polarized light. 500×.

Figure 6-28: $\quad \beta$-quenched $\mathrm{Zr}-4$ sample showing $\alpha$ needles in a parallel or cross pattern. Swab etched and anodized at $15 \mathrm{~V}$. Polarized light. 500×

Figure 6-29: $\quad \alpha$-annealed $\mathrm{Zr}-4$ sample showing much coarser $\alpha$ needles in a parallel or cross pattern. Swab etched and anodized at 15 V. Polarized light. 500×

Figure 6-30: The EDX spectra were obtained in the central positions of the randomly picked precipitates of particle 2 and 6 in the $\alpha$-annealed $\mathrm{Zr}-4$ sample.

Figure 6-31: $\quad$ SEM observations of second phase particles in the $\alpha$-annealed $\mathrm{Zr}-4$ sample. (upper: Secondary electron imaging mode, lower: Backscattering electron imaging mode).

Figure 6-32: The sizes of the second phase particles found in the $\alpha$ - annealed Zircaloy-4 sample (average particle size $=180 \mathrm{~nm}$ ).

Figure 6-33: $\quad$ SEM observations of second phase particles in the as-received Zr-4 sample. (upper: Secondary electron imaging mode, lower: Backscattering electron imaging mode)

Figure 6-34: Comparison of polarization curves for the as-received, $\beta$-quenched and $\alpha$-annealed $\mathrm{Zr}-4$ samples in $0.1 \mathrm{M} \mathrm{B}(\mathrm{OH})_{3}+0.001 \mathrm{M} \mathrm{LiOH}$ with 45 $\mathrm{cm}^{3}(\mathrm{STP}) / \mathrm{kg}\left(\mathrm{H}_{2} \mathrm{O}\right)$ hydrogen. The hydrogen concentration was maintained in the reservoir by suitably controlling the total gas pressure in the system. Scan rate $=1.0 \mathrm{mV} / \mathrm{s}, \mathrm{T}=250^{\circ} \mathrm{C}$ and $=62 \mathrm{bar}$. 
Figure 6-35: The dependence of the steady state current density on the formation potential for the passive films formed on the as-received, $\beta$-quenched and $\alpha$-annealed Zircaloy- 4 samples in $0.1 \mathrm{M} \mathrm{B}(\mathrm{OH})_{3}+0.001 \mathrm{M} \mathrm{LiOH}$ solution with $45 \mathrm{~cm}^{3}(\mathrm{STP}) / \mathrm{kg}\left(\mathrm{H}_{2} \mathrm{O}\right)$ hydrogen. The current was recorded after 24 hours film growth at each potential. The potential was changed in the ascending voltage direction. $\mathrm{T}=250^{\circ} \mathrm{C}$ and $\mathrm{P}=62$ bar.

Figure 6-36: Dependence of the steady state film thickness on the applied potentials for the passive films formed on the as-received, $\beta$-quenched and $\alpha$ annealed zircaloy-4 samples in $0.1 \mathrm{M} \mathrm{B}(\mathrm{OH})_{3}+0.001 \mathrm{M} \mathrm{LiOH}$ solution with $45 \mathrm{~cm}^{3}(\mathrm{STP}) / \mathrm{kg}\left(\mathrm{H}_{2} \mathrm{O}\right)$ hydrogen. The potentials were changed in the negative-to-positive direction. Capacitance was measured after holding potential at each step 24 hours for film growth. $\mathrm{T}=250^{\circ} \mathrm{C}$ and $\mathrm{P}=62$ bar.

Figure 6-37: $\quad$ Impedance spectra for passive film on $\beta$-quenched Zircaloy-4 sample as a function of formation potential in $0.1 \mathrm{M} \mathrm{B}(\mathrm{OH})_{3}+0.001 \mathrm{M} \mathrm{LiOH}$ solutions with $45 \mathrm{~cm}^{3}(\mathrm{STP}) / \mathrm{kg}\left(\mathrm{H}_{2} \mathrm{O}\right)$ hydrogen. The impedance was measured after holding the potential at each potential step 24 hours for film growth. The potentials were changed in negative-to-the positive direction. $\mathrm{T}=250^{\circ} \mathrm{C}$ and $\mathrm{P}=62$ bar.

Figure 6-38: Impedance spectra for the passive films formed on the $\alpha$-annealed Zircaloy-4 sample as a function of formation potential in $0.1 \mathrm{M} \mathrm{B}(\mathrm{OH})_{3}$ $+0.001 \mathrm{M} \mathrm{LiOH}$ solutions with $45 \mathrm{~cm}^{3}(\mathrm{STP}) / \mathrm{kg}\left(\mathrm{H}_{2} \mathrm{O}\right)$ hydrogen. The impedance was measured after holding the potential at each potential step 24 hours for film growth. The potentials were changed from negative-to-the positive direction. $\mathrm{T}=250^{\circ} \mathrm{C}$ and $\mathrm{P}=62$ bar.

Figure 6-39: Impedance spectra for passive films on the as-received Zircaloy-4 sample as a function of formation potential in $0.1 \mathrm{M} \mathrm{B}(\mathrm{OH})_{3}+0.001 \mathrm{M}$ $\mathrm{LiOH}$ solutions with $45 \mathrm{~cm}^{3}(\mathrm{STP}) / \mathrm{kg}\left(\mathrm{H}_{2} \mathrm{O}\right)$ hydrogen. The impedance was measured after holding potential at each potential step 24 hours for film growth. The potentials were changed from negative to positive direction. $\mathrm{T}=250^{\circ} \mathrm{C}$ and $\mathrm{P}=62$ bar.

Figure 6-40: Comparison of the impedance spectra for passive films formed on the as-received, $\beta$-quenched and $\alpha$-annealed zircaloy- 4 samples by neglecting the transient parts in the $0.1 \mathrm{M} \mathrm{B}(\mathrm{OH})_{3}+0.001 \mathrm{M} \mathrm{LiOH}$ solutions with $45 \mathrm{~cm}^{3}(\mathrm{STP}) / \mathrm{kg}\left(\mathrm{H}_{2} \mathrm{O}\right)$ hydrogen. $\mathrm{T}=250^{\circ} \mathrm{C}$ and $\mathrm{P}=62$ bar.

Figure 6-41: Mott-Schottky plots for the passive film formed on the $\beta$-quenched Zircaloy-4 sample at the indicated potentials after stabilization for 24 hours at each point in $0.1 \mathrm{M} \mathrm{B}(\mathrm{OH})_{3}+0.001 \mathrm{M} \mathrm{LiOH}$ solutions with 45 $\mathrm{cm}^{3}(\mathrm{STP}) / \mathrm{kg}\left(\mathrm{H}_{2} \mathrm{O}\right)$ hydrogen. Sinusoidal excitation frequency $=5 \mathrm{kHz}$. Voltage sweep rate in the negative direction from the formation voltage $=100 \mathrm{mV} / \mathrm{s} . \mathrm{T}=250^{\circ} \mathrm{C}$ and $\mathrm{P}=62$ bar. 
Figure 6-42. Mott-Schottky plots for the passive film formed on the $\alpha$-annealed zircaloy-4 sample at the indicated potentials after stabilization for 24 hours at each point in $0.1 \mathrm{M} \mathrm{B}(\mathrm{OH})_{3}+0.001 \mathrm{M} \mathrm{LiOH}$ solutions with 45 $\mathrm{cm}^{3}(\mathrm{STP}) / \mathrm{kg}\left(\mathrm{H}_{2} \mathrm{O}\right)$ hydrogen. Sinusoidal excitation frequency $=5 \mathrm{kHz}$. Voltage sweep rate in the negative direction from the formation voltage $=25 \mathrm{mV} / \mathrm{s} . \mathrm{T}=250^{\circ} \mathrm{C}$ and $\mathrm{P}=62$ bar.

Figure 6-43. Mott-Schottky plots for the passive film formed on the as-received $\mathrm{Zr}-4$ sample at the indicated potentials after stabilization for 24 hours at each point in $0.1 \mathrm{M} \mathrm{B}(\mathrm{OH})_{3}+0.001 \mathrm{M} \mathrm{LiOH}$ solutions with 45 $\mathrm{cm}^{3}(\mathrm{STP}) / \mathrm{kg}\left(\mathrm{H}_{2} \mathrm{O}\right)$ hydrogen. Sinusoidal excitation frequency $=5 \mathrm{kHz}$. Voltage sweep rate in the negative direction from the formation voltage $=25 \mathrm{mV} / \mathrm{s} . \mathrm{T}=250^{\circ} \mathrm{C}$ and $\mathrm{P}=62$ bar.

Figure 6-44. Donor density $\mathrm{N}_{\mathrm{D}}$ of the passive film formed on the as-received, $\beta$ quenched and $\alpha$-annealed Zircaloy- 4 samples at different formation potential in $0.1 \mathrm{M} \mathrm{B}(\mathrm{OH})_{3}+0.001 \mathrm{M} \mathrm{LiOH}$ solutions with 45 $\mathrm{cm}^{3}(\mathrm{STP}) / \mathrm{kg}\left(\mathrm{H}_{2} \mathrm{O}\right)$ hydrogen. $\mathrm{T}=250{ }^{\circ} \mathrm{C}$ and $\mathrm{P}=62$ bar.

Figure 6-45: Polarization curve for $\mathrm{Zr}-2$ in a $0.1 \mathrm{M} \mathrm{B}(\mathrm{OH})_{3}+0.001 \mathrm{M} \mathrm{LiOH}$ with 45 $\mathrm{cm}^{3}(\mathrm{STP}) / \mathrm{kg}\left(\mathrm{H}_{2} \mathrm{O}\right)$ hydrogensolution. The hydrogen concentration was maintained in the reservoir by suitably controlling the total gas pressure in the system. Scan rate $=1.0 \mathrm{mV} / \mathrm{s}, \mathrm{T}=250^{\circ} \mathrm{C}$ and $\mathrm{P}=62$ bar.

Figure 6-46: Impedance spectra for passive film on Zircaloy-2 sample as a function of formation potential in the $0.1 \mathrm{M} \mathrm{B}(\mathrm{OH})_{3}+0.001 \mathrm{M} \mathrm{LiOH}$ solutions with $45 \mathrm{~cm}^{3}(\mathrm{STP}) / \mathrm{kg}\left(\mathrm{H}_{2} \mathrm{O}\right)$ hydrogen. The impedance was measured after holding the potential at each potential step 24 hours for film growth. The potentials were changed in negative-to-the positive direction. $\mathrm{T}=$ $250^{\circ} \mathrm{C}$ and $\mathrm{P}=62$ bar.

Figure 6-47: Comparison of the impedance spectra for passive films formed on the Zircaloy-2 and Zircaloy-4 samples by neglecting the transient parts in the $0.1 \mathrm{M} \mathrm{B}(\mathrm{OH})_{3}+0.001 \mathrm{M} \mathrm{LiOH}$ solutions with $45 \mathrm{~cm}^{3}(\mathrm{STP}) / \mathrm{kg}\left(\mathrm{H}_{2} \mathrm{O}\right)$ hydrogen. $\mathrm{T}=250^{\circ} \mathrm{C}$ and $\mathrm{P}=62$ bar.

Figure 6-48. Mott-Schottky plots for the passive film formed on the Zircaloy-2 sample at the indicated potentials after stabilization for 24 hours at each point in $0.1 \mathrm{M} \mathrm{B}(\mathrm{OH})_{3}+0.001 \mathrm{M} \mathrm{LiOH}$ solutions with 45 $\mathrm{cm}^{3}(\mathrm{STP}) / \mathrm{kg}\left(\mathrm{H}_{2} \mathrm{O}\right)$ hydrogen. Sinusoidal excitation frequency $=5 \mathrm{kHz}$. Voltage sweep rate in the negative direction from the formation voltage $=100 \mathrm{mV} / \mathrm{s} . \mathrm{T}=250^{\circ} \mathrm{C}$ and $\mathrm{P}=62$ bar.

Figure 6-49. Donor density $\mathrm{N}_{\mathrm{D}}$ of the passive films formed on the Zircaloy-2 and Zircaloy-4 samples at different formation potential in $0.1 \mathrm{M} \mathrm{B}(\mathrm{OH})_{3}+$ $0.001 \mathrm{M} \mathrm{LiOH}$ solutions with $45 \mathrm{~cm}^{3}(\mathrm{STP}) / \mathrm{kg}\left(\mathrm{H}_{2} \mathrm{O}\right)$ hydrogen. $\mathrm{T}=250$ ${ }^{\circ} \mathrm{C}$ and $\mathrm{P}=62$ bar. 


\section{LIST OF TABLES}

Table 1-1. Base case input variables for the pores.

Table 1-2. Bulk and pore concentration, and $\mathrm{PH}$ for this environment

Table 2-1. Rate constants for the four primary interfacial reactions under PWR conditions

Table 2-2. Rate constants for the five primary interfacial reactions under BWR coolant conditions

Table 2-3. Parameter values after optimization of the point defect model on the experimental impedance data for zirconium in de-aerated hydrogen-added aqueous solution $\left(0.1 \mathrm{M} \mathrm{B}(\mathrm{OH})_{3}+0.001 \mathrm{M} \mathrm{LiOH}\right.$ solution with $22 \mathrm{~cm}^{3}$ $(\mathrm{STP}) / \mathrm{kg}\left(\mathrm{H}_{2} \mathrm{O}\right)$ hydrogen at $\left.250^{\circ} \mathrm{C}, \mathrm{pH}=6.94\right)$.

Table 2-4. Parameter values after optimization of the point defect model on the experimental impedance data for zirconium in de-aerated aqueous solution $\left(0.1 \mathrm{M} \mathrm{B}(\mathrm{OH})_{3}+0.001 \mathrm{M} \mathrm{LiOH}\right.$ solution at $\left.250^{\circ} \mathrm{C}, \mathrm{pH}=6.94\right)$

Table 6-1. Chemical specification for Zircaloy-4 sample determined by Electron Probe Micro Analysis (EPMA), in wt $\%$

Table 6-2. Second phase particles in Zircaloy-4 observed by SEM

Table 6-3: Chemical specification for Zircaloy-2 samples determined by Electron Probe Micro Analysis (EPMA), in wt\%

Table 6-4. Calculated potentials for the $\mathrm{Ag} / \mathrm{AgCl}$ (sat. $\mathrm{KCl}$ ) reference electrode vs. the Standard Hydrogen Electrode (SHE) at elevated temperature. 


\section{INTRODUCTION}

With the development of higher burn-up fuels for nuclear power reactors, much greater demands are being placed on the performance of the Zircaloy fuel sheaths. The principal threat to the integrity of the sheath is oxidation/corrosion and hydriding, leading to more-or-less uniform thinning and, in some instances, to localized corrosion in the form of nodular attack and/or hydriding. Failure leads to the release of fission products into the coolant, which in turn contributes to the man-REM costs of operating the system. Extensive fuel failures may require shutdown, which results in the unit being unavailable for normal operation. Thus, strong operational and economic reasons exist for enhancing fuel reliability.

While the kinetics and mechanisms of the oxidation of zirconium and zirconium alloys have been extensively studied, little effort has been made to develop deterministic (as opposed to empirical and semi-empirical) models that can be used to predict fuel sheath performance and reliability at high burns-ups in operating reactors. Those attempts at developing algorithms have employed semi-empirical, parabolic or cubic models to extrapolate oxide thickness data to longer times, but the validity of these models is highly uncertain. Because there are lacks of a sound electrochemical basis for corrosion process under free corrosion conditions.

Our theories and models will be based on recent advances that we have made in discerning the mechanisms of oxidation of metals and alloys, in general, as well as on advanced models that we contribute to develop to describe the electrochemistry of reactor coolants at the fuel sheath/coolant interface in terms of the bulk coolant chemistry and the operating condition in the reactor. In addition, we develop the theory of passivity and passivity breakdown, in the form of the Point Defect Model, with the emphasis on zirconium and zirconium alloys in reactor coolant environments, the continued development of a code that can be used by reactor operators to actively manage the accumulation of corrosion damage to the fuel cladding in both BWRs and PWRs. A particularly important aspect of the boiling crevices to describe the concentration of solutes in porous deposits (CRUD) on fuel under boiling is actually in contact with the Zircaloy cladding.

\section{OBJECTIVES AND ACCOMPLISHMENTS}

The proposed work seeks to develop a comprehensive model that can be used to predict performance, and to assess the risk of failure, of Zircaloy fuel cladding in commercial BWRs and PWRs under high burn-up conditions. During this fifth quarter (January 1 to April 31, 2004), the work has involved the following tasks:

\section{Task 1. Definition of the Interfacial Environment.}


Objectives: In this initial task, we have modified the Boiling Crevice Model to describe the evolution of the environment in CRUD pores and hence in contact with the Zircaloy surface under low super heat (nucleate boiling, PWRs) and high super heat (sustained boiling, BWRs). Because the BCM also contains the Mixed Potential Model, it is possible to calculate the $\mathrm{pH}$ and the ECP (corrosion potential) at the Zircaloy/environment interface within the pores. These values, which are expected to be significantly different from the bulk values, will then be used to model the oxidation of zirconium.

Task Status: The task has been essentially completed, with the work in the first instance involving modification of the boiling crevice model that was developed previously to describe the evolution of chemical conditions within tube/tube sheet crevices in PWR steam generators. In the present case, the model has been modified to describe the evolution of chemical and electrochemical conditions within porous CRUD deposits on nuclear fuel sheaths under sustained boiling (BWRs) and nucleate boiling (PWRs) conditions.

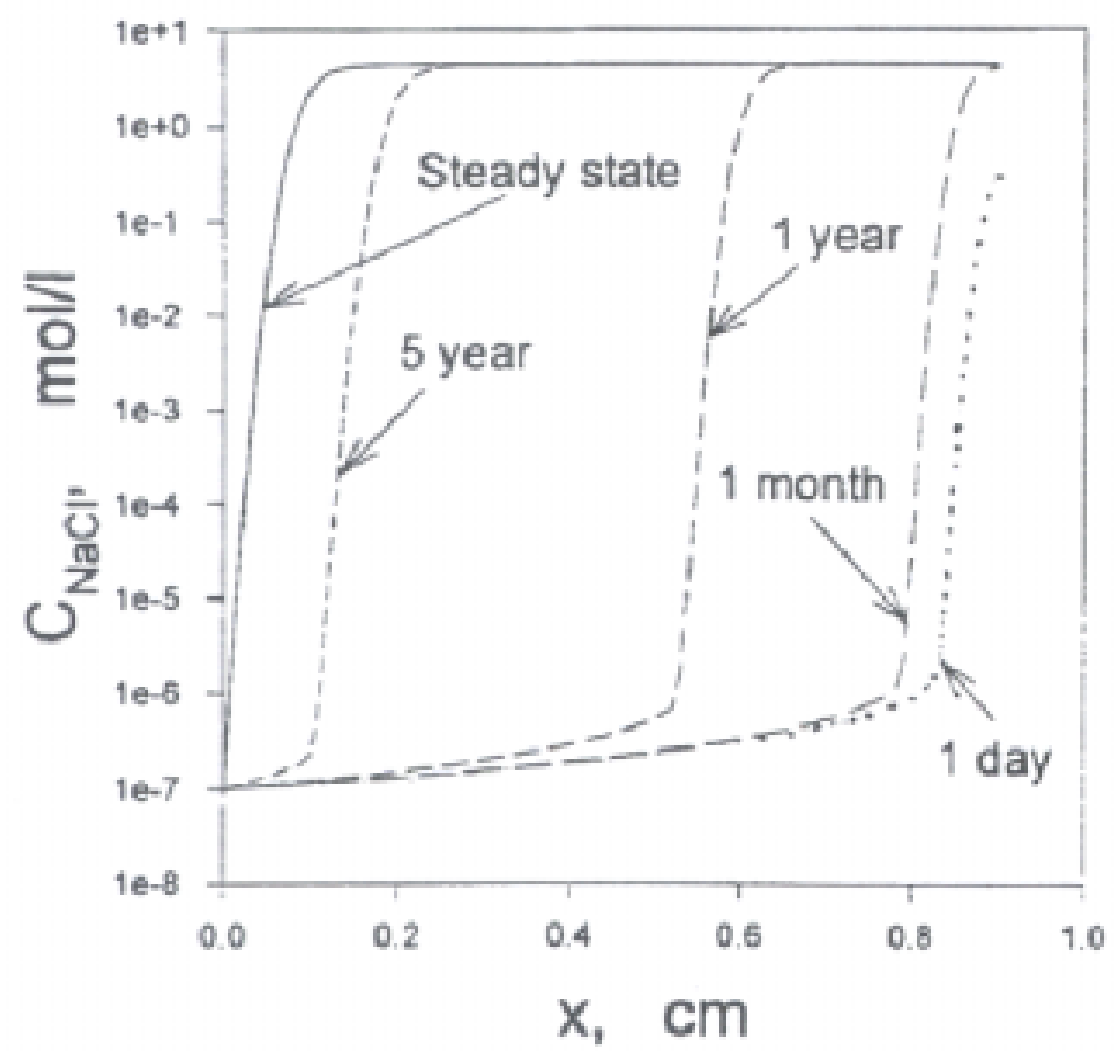

Figure 1-1. Predicted build up of a concentrated solution in a boiling, 1$\mathrm{cm}$ long crevice with time for a bulk $\mathrm{NaCl}$ concentration of 10-7 M (5.8 $\mathrm{ppb}$ ) and a superheat of $28^{\circ} \mathrm{C}$. Note that the concentrated, stationary phase progressively fills the crevice as time increases and ultimately (in the 
steady state) occupies the entire crevice except for a small region at the crevice mouth [1].

The Boiling Crevice Model calculates the evolution of the solution contained within a boiling cavity (e.g., within the pores of a porous CRUD layer on the fuel) by noting that the solubility of electrolytes in steam is much less than in liquid water, so that as boiling occurs within the crevice the concentration of the electrolyte increases. However, the concentration process begins at the bottom of the pore where the temperature is highest. Thus, a concentrated solution is produced in the pore from the pore base and it gradually expands to fill the pore, as shown in Figure 1-1 for a 1-cm deep pore. The concentration of the solution is determined by the super heat, such that the elevation in boiling temperature at the steady state concentration matches the super heat. Physically, the process produces a "simmering", stationary fluid in the pores that can concentrate electrolytes by a factor of more than $10^{7}$. This process is of fundamental importance in PWR operation, because it is believed to be the mechanism for concentrating $\mathrm{Li}^{+}$and $\mathrm{B}(\mathrm{OH})_{4}{ }^{-}$in CRUD pores on the fuel and ultimately of the precipitation of $\mathrm{LiB}(\mathrm{OH})_{4}$. The high boron concentration absorbs neutrons to the extent that fission ceases and the power is drastically reduced. This phenomenon is known as the "axial offset anomaly". As seen from Figure 1-1, the build up can occur over extended periods of time, but we note that the length of the pore chosen for these calculations is much greater than those that exist in CRUD on the fuel (we have yet to determine the actual pore length in the CRUD).

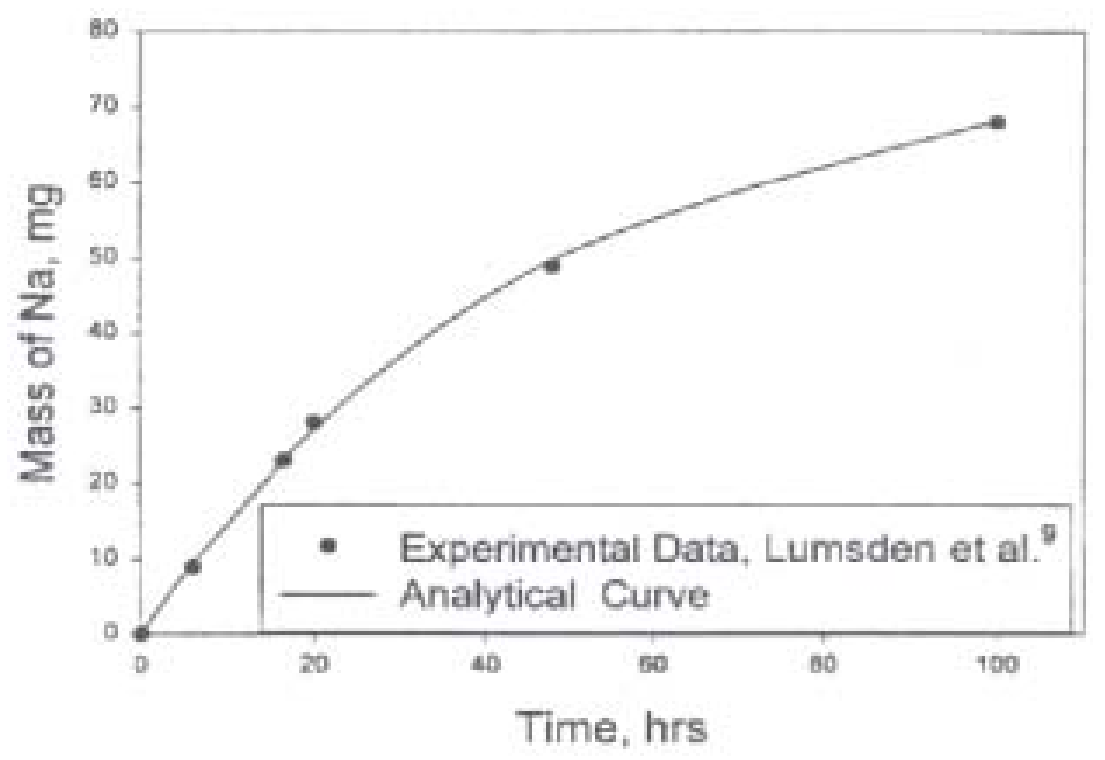

Figure 1-2. Comparison between theory and experiment for the average volume concentration of $\mathrm{Na}^{+}$in a boiling crevice in contact with a bulk solution containing $40 \mathrm{ppm} \mathrm{NaOH}$ [1]. 
That the BCM produces realistic results is shown by the data plotted in Figure 1-2, where the mass of $\mathrm{Na}^{+}$concentrated in the crevice is compared with experimental data published by Lumsden for a crevice of identical dimensions. In performing this comparison, we fit the model to the first two experimental data points, in order to determine two model parameters, the values of which were unknown for this system. Comparison of the model with the experimental data for longer times shows excellent agreement, thereby lending great credence to the veracity of the model.

The theory was fit to the first two experimental points. The experimental data are from Lumsden, et al. [2].

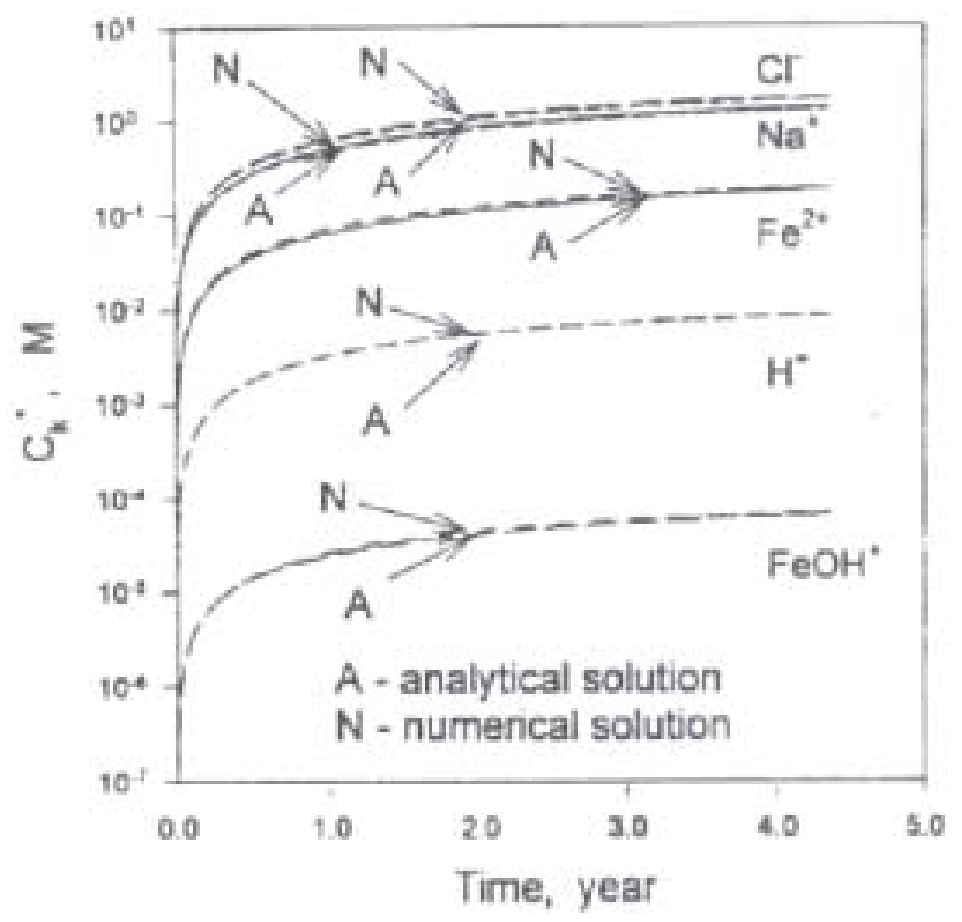

Figure 1-3. Volume averaged concentrations as a function of time for a heated crevice with a superheat of $28^{\circ} \mathrm{C}$, a bulk concentration of $\mathrm{NaCl}$ of $10^{-7} \mathrm{M}$, and a bulk system temperature of $280^{\circ} \mathrm{C}$. The iron species are formed by corrosion reactions in the crevice $[1,3]$.

In the work on PWR Steam Generator crevices, we developed an approximate analytical solution to the mass transport, electrolyte concentration mechanism that provides a fast method for performing the calculations. We have tested the approximate method extensively, and shown that it predicts crevice concentrations and electrochemical parameters within the crevice (e.g., the corrosion potential) to within a few tenths of one percent of the more time-consuming, numerical solution of the 
transport equations (Figure 1-3). This is an important development, because the eventual simulation of the processes that occur on the fuel, including those that are responsible for mass and activity transport and the axial offset anomaly, will require many thousands of runs of the algorithm in order to describe the evolution of the system over a typical operating history of a reactor.

Returning now to Figure 1-3, we see that, for the assumed conditions of $[\mathrm{NaCl}]=$ $10^{-7} \mathrm{M}$ in the bulk solution, for a super heat of $28^{\circ} \mathrm{C}$, and for a bulk temperature of $280^{\circ} \mathrm{C}$, the crevice solution concentrates by a factor of about $10^{7}$ and evolves toward an impure $\mathrm{NaCl}$ brine that is contaminated with $\mathrm{Fe}^{2+}$ species from corrosion (in this case). Note that $90 \%$ of the enhanced concentration within the crevice is predicted to occur within the first year.

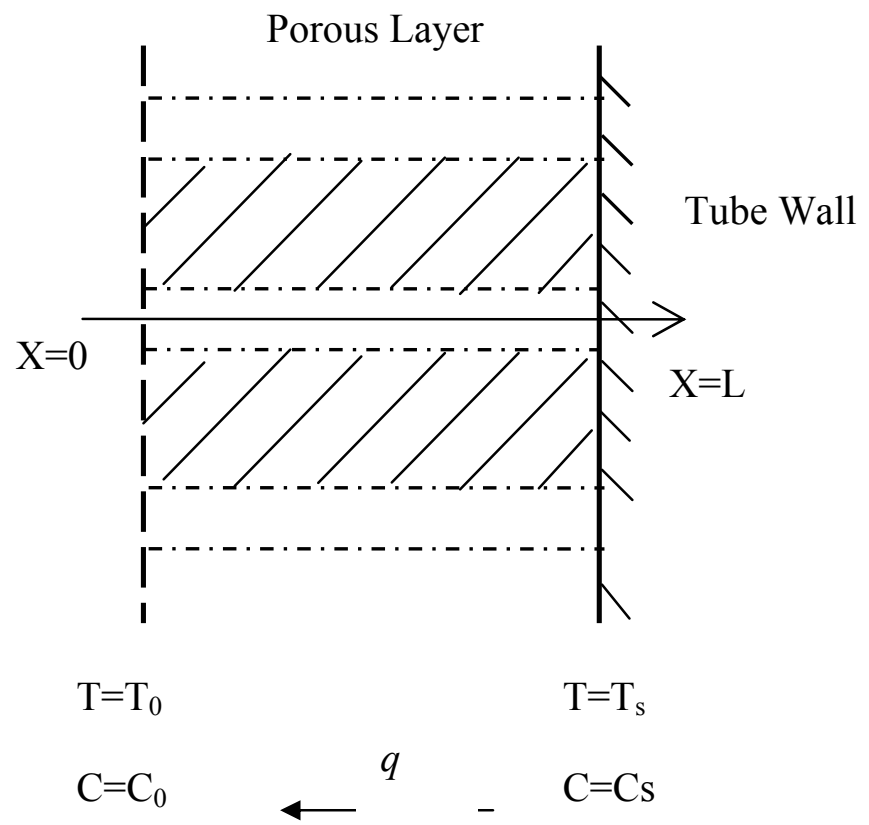

Figure 1-4 Schematic illustration of the modified boiling crevice model

The cladding is normally covered with a layer of porous CRUD ("Chalk River Unidentified Deposit"). Thus, under boiling (BWR) and nucleate (PWR) operating conditions, electrolytes in the bulk coolant become concentrated in the pores. Accordingly, the environment that is in contact with Zircaloy surface is considerably different from that of the bulk as noted previously. By considering the "chemical amplifier" effects of these pores on the concentration that is in contact with cladding surface, we proposed the modified boiling crevice model. The model is illustrated in Figure 1-4. In Figure 1-4, we consider a single pore and the crud immediately around it.

The molar flux (in the $\mathrm{x}$ direction) of species $k$ can be written as: 


$$
J_{k}=-D_{s} \frac{d C_{k}}{d x}+C_{k} v
$$

where $C_{k}$ is the concentration, $D_{k}$ is the superficial diffusion coefficient, $v$ is the superficial velocity for a given cross-section of the porous medium. By assuming the inlet and outlet fluxes in the pore are balanced, i.e., $\mathrm{J}=0$, Equation (1-1) can be written as

$$
D_{s} \frac{d C_{k}}{d x}=C_{k} v
$$

Rewriting the above equation in the format as $\frac{d C_{k}}{C_{k}}=\frac{v}{D_{s}} d x$ and integrate both sides, we get the concentration as a function of distance to the opening of pore.

$$
C=C_{0} \exp \left(\frac{v x}{D_{s}}\right)
$$

At $\mathrm{X}=\mathrm{L}$, the concentration $\mathrm{Cs}$ in contact with the zirconium tubing can be obtained from above equation as:

$$
C_{s}=C_{0} \exp \left(\frac{v L}{D_{s}}\right)
$$

The concentration factor can be obtained as:

$$
C F \equiv \frac{C_{s}}{C_{0}}=\exp \left(\frac{v L}{D_{s}}\right)
$$

where, only the velocity $v$ is unknown, which can be calculated from the heat conservation:

$$
q_{v} S_{v}=q S
$$

as $V=S L, V_{v}=S L$, and $\varepsilon=\frac{V_{v}}{V}$, so

$$
q_{v}=q \frac{S}{S_{v}}=q \frac{V}{V_{v}}=\frac{q}{\varepsilon}
$$

and

$$
q_{v}=h \rho v
$$

From Equations (1-7) and (1-8), we find the velocity as: 


$$
v=\frac{q_{v}}{h \rho}=\frac{q}{h \rho \varepsilon}
$$

By substituting Equation (1-9) into Equation (1-5), we get the concentration factor as:

$$
C F \equiv \frac{C_{s}}{C_{0}}=\exp \left(\frac{v L}{D}\right)=\exp \left(\frac{q L}{h \rho \varepsilon D}\right)
$$

The concentrations of lithium and borate ions that govern whether a precipitate will form are the bulk concentrations multiplied by the respective concentration factors as determined from Equation (1-10). Non-volatile species tend to concentrate in a porous deposit layer overlaying a boiling surface. By using representative data (Table 1-1) available from literature, we calculate the concentrations of lithium and borate ions using the model described above. Determination of the concentration factor requires knowledge of the diffusion coefficients of these species. By assuming $D_{L^{+}}=1.11 * 10^{-3} \mathrm{~cm}^{2} / \mathrm{s}$ for $\mathrm{Li}^{+}$and $D_{\mathrm{B}(\mathrm{OH}) 4-}=2.89 * 10^{-4} \mathrm{~cm}^{2} / \mathrm{s}$ for $\mathrm{B}(\mathrm{OH})_{4}{ }^{-}$at the incore temperature $\left(345^{\circ} \mathrm{C}\right)$ of a PWR [5], we obtain

$$
\begin{aligned}
& C F_{L i}=\exp \left(\frac{100 * 10^{-2}}{1557.5 * 0.755 * 0.8 * 1.11 * 10^{-3}}\right)=2.6 \\
& C F_{B(\mathrm{OH})_{4}}=\exp \left(\frac{100 * 10^{-2}}{1557.5 * 0.755 * 0.8 * 2.89 * 10^{-4}}\right)=39.6
\end{aligned}
$$

Table 1-1 Base case input variables for the pores.

\begin{tabular}{|c|c|c|}
\hline Parameter & Value & Source \\
\hline $\mathrm{h}$ & $1.557 \mathrm{~J} / \mathrm{g}$ & $(3)$ \\
\hline$\rho$ & $0.755 \mathrm{~g} / \mathrm{cm}^{3}$ & $(3)$ \\
\hline$\varepsilon$ & 0.8 & $(3,5)$ \\
\hline $\mathrm{L}$ & $100 \mu \mathrm{m}$ & $(4)$ \\
\hline $\mathrm{q}$ & $100 \mathrm{~W} / \mathrm{cm}^{2}$ & $(4)$ \\
\hline
\end{tabular}

Table 1-2 Bulk and pore concentration, and $\mathrm{PH}$ for this environment

\begin{tabular}{|c|c|c|c|}
\hline & {$\left[\mathrm{B}(\mathrm{OH})_{3}\right](\mathrm{ppm})$} & {$[\mathrm{LiOH}](\mathrm{ppm})$} & $\mathrm{pH}$ \\
\hline $\mathrm{C}$ & 1000 & 2 & 7.92 \\
\hline $\mathrm{CF}^{*} \mathrm{C}$ & 39600 & 5.2 & 5.71 \\
\hline
\end{tabular}

The concentration factor for the species $\mathrm{B}(\mathrm{OH})_{4}{ }^{-}$is almost 40 , which means the concentration of $\mathrm{B}(\mathrm{OH})_{4}{ }^{-}$at the bottom of the pores is 40 times larger than that in the 
bulk. Typical PWR water primary coolant contains $1000 \mathrm{ppm}\left[\mathrm{B}(\mathrm{OH})_{3}\right]$ and $2 \mathrm{ppm}$ [LiOH]. The concentrations of $\mathrm{Li}^{+}$and $\mathrm{B}(\mathrm{OH})_{4}{ }^{-}$in the pores and $\mathrm{pH}$ calculated from the model are shown in Table 1-2. We can see that the concentration changed a lot for $\mathrm{B}(\mathrm{OH})_{4}{ }^{-}$and the $\mathrm{pH}$ changed from 7.94 to 5.71 at $345^{\circ} \mathrm{C}$ and this is the actual environment in contact with the zircaloy cladding.

Issues and Concerns: None.

\section{References:}

[1] G. R. Engelhardt, D. D. Macdonald, P. J. Millett, Corrosion Science 41 2191-2211. (1999)

[2] J. B. Lumsden, G. A. Pollok, P. J. Millett, C. Fauchon, Proceeding of the VIII International Symposium on Environmental Degradation of Materials in Nuclear Power Systems Water Reactor, Amelia Island, August, (1997)

[3] G. R. Engelhardt, D. D. Macdonald, P. J. Millett, Corrosion Science 41 2165-2190. (1999)

[4] R. V. Macbeth, "Boiling on Surface Overlayer with a Porous Deposit: Heat Transfer Rates Obtainable by Capillary Action”. AEEW-R. 711, W8958. (1971)

[5] Frattini, P.L., J. Blok, S. Chauffriat, J. Sawicki, and J. Riddle, Nuclear Energy-Journal of the British Nuclear Energy Society, 40(2): p. 123-135. (2001)

\section{Task 2. PDM Development.}

\subsection{PDM}

Objectives: The objectives of this task is to develop a Point Defect Model (PDM) [1] for the growth of bi-layer passive films on zirconium and Zircaloys under PWR and BWR coolant conditions, where hydride and oxide barrier layers form, respectively, both of which are covered by a porous outer layer.

Task Status: We have modified the current PDM [1] and developed two models for the growth of passive films on zirconium according to the prevailing reactor coolant conditions (PWR or BWR). In the previous report, we showed that the derived equations for the electrochemical parameters and some results for predicting the barrier layer thickness as a function of the applied voltage, $\mathrm{pH}$, porosity, and temperature for both BWR and PWR primary water chemistry conditions.

In order to devise a deterministic basis for describing the oxidation phenomenon, our model includes the contribution of the cathodic processes (reduction of oxygen and hydrogen evolution) to the conversation of charge. Because the cathodic reactions, which must occur at the same rate as the zirconium oxidation reaction, may actually control the overall rate, their inclusion in the models is a matter of great importance. Most importantly, the kinetics of the cathodic reactions appear to depend critically on the 
density and size of second phase particles (SPPs) in the passive film as the particles may act as low resistance, electronic pathways through an otherwise high resistance passive film. The models have now been developed to yield mathematical expressions for the impedance of oxidized zirconium and Zircaloys as a function of voltage and other experimental variables. The impedance model parameter values have been extracted from electrochemical impedance spectroscopic data for zirconium in both deaerated and hydrogenated, borate buffer aqueous solutions ( $\mathrm{pH} 6.94$ ) at $250^{\circ} \mathrm{C}$ by optimization.

\section{PWR Coolant Conditions}

Under open circuit conditions, all interfacial reactions that produce or consume electrons contribute to the total steady-state current density, $I_{T}$. Thus, with reference to Figure 2-1 we write:

\begin{tabular}{|c|c|c|c|}
\hline $\mathrm{Zr}$ & $\mathrm{ZrH}_{2-\mathrm{x}}$ Barrier layer & $\mathrm{ZrO}_{2}$ Outer layer & Solution \\
\hline $\begin{array}{r}\text { (1) } Z r+V_{Z r}^{2-} \\
\text { (2) } Z r-\end{array}$ & $\begin{aligned} & \stackrel{k_{1}}{\longrightarrow} \mathrm{Zr}_{\mathrm{Zr}}+2 e^{-}(4) \mathrm{Zr}_{\mathrm{Zr}}+\mathrm{H}_{2} \mathrm{O} \\
& \stackrel{k_{2}}{\longrightarrow} \mathrm{Zr}_{i}^{2+}+2 e^{-} \\
&(5) \mathrm{Zr}_{i}^{2+}+\mathrm{H}_{2} \mathrm{O} \\
& \stackrel{k_{3}}{\longrightarrow} \mathrm{Zr}_{\mathrm{Zr}}+2 \mathrm{~V}_{\mathrm{H}}^{\bullet}+2 e^{-} \\
&(6) V_{H}^{\bullet}+\mathrm{H}_{2} \mathrm{O}+2 e^{-} \\
&(7) 2 \mathrm{H}_{2} \mathrm{O}+2 e^{-} \\
& \\
& \text {(8) } 2 \mathrm{H}^{+}+2 e^{-} \\
& \text {(9) } \mathrm{ZrH}_{2}+4 \mathrm{OH}\end{aligned}$ & 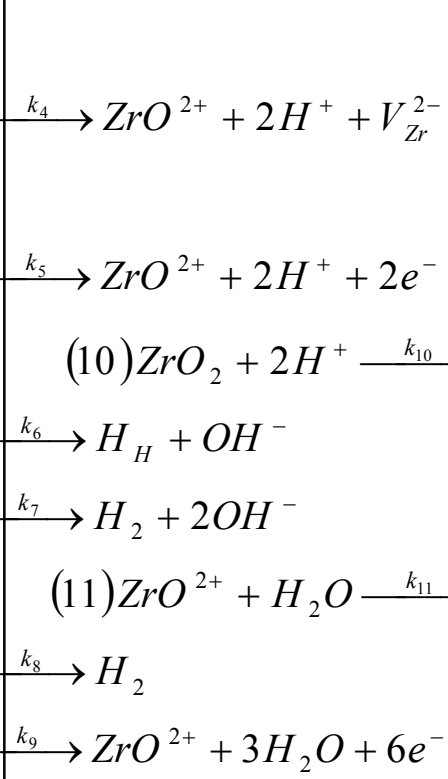 & $\begin{array}{l}\rightarrow \mathrm{ZrO}^{2+}+\mathrm{H}_{2} \mathrm{O} \\
\rightarrow \mathrm{ZrO}_{2}+2 \mathrm{H}^{+}\end{array}$ \\
\hline
\end{tabular}

Figure 2-1. Interfacial reactions leading to the generation and annihilation of point defects within the barrier layer and to the formation of both the barrier layer and outer layer of the passive film on zirconium under PWR coolant condition. $Z r=$ zirconium atom, $Z r_{Z r}$ = zirconium cation in a normal cation site on the metal sublattice in the barrier layer, $V_{Z r}^{2-}=$ cation vacancy on the metal sublattice in the barrier layer, $Z r_{i}^{2+}=$ zirconium interstitial in the barrier layer, $V_{H}^{\bullet}=$ hydrogen anion vacancy on the anion sublattice of the barrier layer, $H_{H}=$ hydrogen anion in an anion site on the anion sublattice of the barrier layer. 


$$
I_{T}=F \cdot\left[\begin{array}{l}
2 k_{1} \cdot a_{V_{Z r}^{2-}}^{M B I}+2 k_{2}+2 k_{3}+2 \theta \cdot k_{5} \cdot a_{Z r_{i}^{2+}}^{B O I} \cdot a_{W}^{B O I}+6 \theta \cdot k_{9} \cdot\left(a_{O H^{-}}^{B O I}\right)^{m} \\
-2 \theta \cdot k_{6} \cdot a_{V_{H}^{B}}^{B O I} \cdot a_{W}^{B O I}-2 \theta \cdot k_{7} \cdot\left(a_{W}^{B O I}\right)^{2}-2 \theta \cdot k_{8} \cdot\left(a_{H^{+}}^{B O I}\right)^{n}
\end{array}\right]
$$

where $\theta$ is the porosity of the outer layer; $a_{w}^{B O I}$ is the activity of water at the barrier layer/outer layer interface (BOI); $a_{V_{Z_{r}}^{2-}}^{M B I}$ is the activity of the $V_{Z r}^{2-}$ at the metal/barrier layer interface (MBI); $a_{Z r_{i}^{r^{+}}}^{B O I}$ and $a_{V_{H}^{*}}^{B O I}$ are the activities of the $Z r_{i}^{2+}$ and $V_{H}^{\bullet}$ on the sublattice of the barrier layer at the BOI, respectively; and $a_{H^{+}}^{B O I}$ is the activity of $H^{+}$in water at the BOI, respectively; and $m$ and $n$ are kinetic orders. (Note that the outer layer is porous).

From activated complex theory, the rate constants $k_{j}$ for the elementary defect generation and annihilation reactions, and other reactions, at the interfaces, as functions of the potential drops at the MBI and BOI, are given by:

$$
\begin{aligned}
& k_{1}=k_{1}^{0} \cdot e^{\alpha_{1} \cdot \gamma \cdot \phi_{M B I}} \\
& k_{2}=k_{2}^{0} \cdot e^{\alpha_{2} \cdot \gamma \cdot \phi_{M B I}} \\
& k_{3}=k_{3}^{0} \cdot e^{\alpha_{3} \cdot \gamma \cdot \phi_{M B I}} \\
& k_{4}=k_{4}^{0} \cdot e^{-\Delta G_{4}^{\mp} / R T} \\
& k_{5}=k_{5}^{0} \cdot e^{-\Delta G_{5}^{\mp} / R T} \\
& k_{6}=k_{6}^{0} \cdot e^{-\alpha_{6} \cdot \gamma \cdot \phi_{B O I}} \\
& k_{7}=k_{7}^{0} \cdot e^{-\alpha_{7} \cdot \gamma \cdot \phi_{B O I}} \\
& k_{8}=k_{8}^{0} \cdot e^{-\alpha_{8} \cdot \gamma \cdot \phi_{B O I}} \\
& k_{9}=k_{9}^{0} \cdot e^{\alpha_{9} \cdot \cdot \cdot \phi_{B O I}}
\end{aligned}
$$

where $\gamma=F / R T, F$ is Faraday's constant, $R$ is gas constant, $T$ is the Kelvin temperature, and $\alpha_{j}$ is the dimensionless transfer coefficient, having values of 0 (initial state) $<\alpha_{j}<1$ (final state), $k_{j}^{0}(\mathrm{j}=1,2,3,6,7,8,9)$ is the standard rate constant for the $j^{\text {th }}$ elementary reaction, and $\Delta G_{j}(\mathrm{j}=4,5)$ is standard Gibbs free energy of activation. Note that, because of the high electro negativity of zirconium, the system is assumed to be far from equilibrium and, consequently, the reverse reactions in Figure 2-1 are not considered. Accordingly, the potential drops at the metal/barrier layer interface (MBI), $\phi_{M B I}$, and the barrier/outer layer interface (BOI), $\phi_{B O I}$, are crucial quantities in determining the rates of the elemental reactions in the system. 
Under steady-state conditions, the fluxes of all the species are constant. Accordingly, by equating the rates of formation and annihilation of each defect at the respective interfaces, it is possible to develop relationships between the rate constants for the reactions at the MBI and BOI. In other words, the rate of generation of zirconium vacancies at the BOI [Reaction (4) in Figure 2-1] should be the same as the rate of annihilation of zirconium vacancies at the MBI [Reaction (1) in Figure 2-1] yielding:

$$
a_{V_{Z r}^{2-}}^{M B I} \cdot k_{1}=\theta \cdot a_{W}^{B O I} \cdot k_{4}
$$

Under steady state conditions, the generation rate of zirconium interstitials at the MBI [Reaction (2) in Figure 2-1] must be the same as the annihilation rate of zirconium interstitials at the BOI [Reaction (5) in Figure 2-1]. The rates of these two reactions are expressed by Equations 2-3 and 2-6, respectively, and setting the rates equal yields

$$
k_{2}=\theta \cdot a_{Z r_{i}^{2+}}^{B O I} \cdot a_{W}^{B O I} \cdot k_{5}
$$

Likewise, the generation rate of hydrogen vacancies at the MBI [Reaction (3) in Fig. 2-1] must equal the annihilation rate of the same species at the BOI [Reaction (6) in Fig. 2-1]. In addition, the rates of these two reactions can be expressed by Equations 2-4 and 2-7, which yield

$$
2 k_{3}=\theta \cdot a_{V_{H}^{\dot{H}}}^{B O I} \cdot a_{W}^{B O I} \cdot k_{6}
$$

We assume the solution in the pore interior is dilute, so that $a_{W}^{B O I}$ is taken to be 1. Substitution of Equation (2-13) into Equation (2-1) therefore yields:

$$
I_{T}=F \cdot\left[2 k_{1} \cdot a_{V_{Z r}^{4-}}^{M B I}+4 k_{2}-2 k_{3}+6 \theta \cdot k_{9}\left(a_{O H^{-}}^{B O I}\right)^{m}-2 \theta \cdot k_{7}\left(a_{W}^{B O I}\right)^{2}-2 \theta \cdot k_{8}\left(a_{H^{+}}^{B O I}\right)^{n}\right\rfloor(2-1
$$

According to Equations (2-2) to (2-4), (2-7), and (2-13), we can write

$$
k_{i}=k_{i}^{0} \cdot\left[k_{3} / k_{3}^{0}\right]^{\alpha_{i} / \alpha_{3}}=k_{i}^{0} \cdot\left[a_{V_{H}^{*}}^{B O I} \cdot(\theta / 2) \cdot k_{6} / k_{3}^{0}\right]^{\alpha_{i} / \alpha_{3}} \quad(i=1,2)
$$

Substitution of Equations (2-8), (2-9), and (2-15) into Equation (2-14) yields (by assuming the $a_{W}^{B O I}=1$ ):

$$
I_{T}=F\left[\begin{array}{l}
2 k_{1}^{0} a_{V_{Z_{r}}^{4}}^{M B I}\left[a_{V_{H}^{*}}^{B O I}(\theta / 2) \cdot k_{6} / k_{3}^{0}\right]^{\alpha_{1} / \alpha_{3}}+4 k_{2}^{0}\left[a_{V_{H}^{*}}^{B O I}(\theta / 2) \cdot k_{6} / k_{3}^{0}\right]^{\alpha_{2} / \alpha_{3}}-a_{V_{H}^{*}}^{B O I} \theta \cdot k_{6} \\
+6 \theta \cdot\left(a_{O H}^{B O I}\right)^{m} k_{9}^{0} \cdot e^{\alpha_{9} \cdot \cdot \cdot \phi_{B O I}}-2 \theta \cdot k_{7}^{0} \cdot e^{-\alpha_{1} \cdot \cdot \cdot \phi_{B O I}}-2 \theta \cdot k_{8}^{0} \cdot\left(a_{H^{+}}^{B O I}\right)^{n} e^{-\alpha_{8} \cdot \cdot \cdot \phi_{B O I}}
\end{array}\right]
$$

In order to simplify Equation (2-16), we make the following assumptions.

and

$$
\alpha_{1}=\alpha_{2}=\alpha_{3}
$$




$$
\alpha_{6}=\alpha_{7}=\alpha_{8}
$$

Thus, under open circuit conditions, the total, partial anodic and cathodic steadystate current densities on the surface of the working electrode are as follows:

$$
\begin{aligned}
& \left.I_{T}=F \theta \cdot\left[a_{V_{H}^{*}}^{B O I}\left(k_{1}^{0} \cdot a_{V_{\frac{2}{t r}}}^{M B I} / k_{3}^{0}+2 k_{2}^{0} / k_{3}^{0}-1\right) \cdot k_{6}^{0}-\left(2 k_{7}^{0}+2 k_{8}^{0}\left(a_{H^{+}}^{B O I}\right)^{n}\right)\right] \cdot e^{-\alpha_{6} \cdot \cdot \phi_{B O I}}+6 k_{9}^{0}\left(a_{O H}^{B O I}\right)^{m} e^{\alpha_{9} \cdot \cdot \cdot \phi_{B O I}}\right]
\end{aligned}
$$

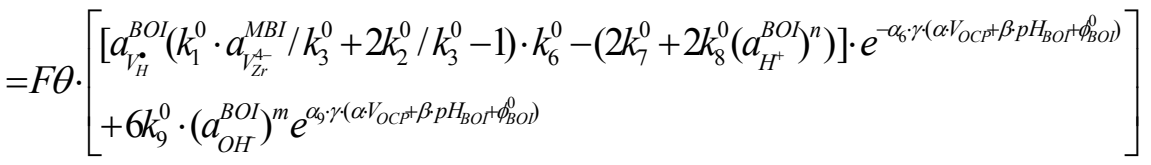

$$
\begin{aligned}
& =F \theta \cdot\left[(a-b) \cdot e^{-\alpha_{6} \cdot \cdot \cdot \cdot V_{O C P}}+c \cdot e^{\alpha_{9} \cdot \cdot \cdot \alpha V_{O C P}}\right] \\
& I_{a}=F \cdot \theta \cdot\left(a \cdot e^{-\alpha_{6} \cdot \gamma \cdot \alpha \cdot V_{O C P}}+c \cdot e^{\alpha_{9} \cdot \gamma \cdot \alpha \cdot V_{O C P}}\right)
\end{aligned}
$$

and

$$
I_{c}=\left|I_{T}-I_{a}\right|=F \cdot \theta \cdot b \cdot e^{-\alpha_{6} \cdot \gamma \cdot \alpha \cdot V_{O C P}}
$$

where $\alpha$ is the polarizability of the BOI; $\beta$ is the dependence of the voltage drop across the BOI on the local $\mathrm{pH}\left(\mathrm{pH}_{B O I}\right)$, and $\phi_{B O I}^{0}$ is a constant. $V_{O C P}$ is the open circuit potential. $\phi_{B O I}$ is a function of these parameters. We will derive the equations later.

$$
\begin{aligned}
& a=a_{V_{H}^{+}}^{B O I} \cdot\left(k_{1}^{0} \cdot a_{V_{Z_{r}}^{4-}}^{M B I} / k_{3}^{0}+2 \mathrm{k}_{2}^{0} / \mathrm{k}_{3}^{0}+1\right) \cdot k_{6}^{0} \cdot e^{-\alpha_{6} \cdot \gamma \cdot\left(\beta \cdot p H_{B O I}+\phi_{B O I}^{0}\right)} \\
& b=\left(2 a_{V_{H}^{\dot{B}}}^{B O I} \cdot k_{6}^{0}+2 k_{7}^{0}+2 k_{8}^{0} \cdot\left(a_{H^{+}}^{B O I}\right)^{n}\right) \cdot e^{-\alpha_{6} \cdot \gamma \cdot\left(\beta \cdot p H_{B O I}+\phi_{B O I}^{0}\right)} \\
& c=6 k_{9}^{0} \cdot\left(a_{O H^{-}}^{B O I}\right)^{m} e^{\alpha_{9} \cdot \gamma \cdot\left(\beta \cdot p H_{B O I}+\phi_{B O I}^{0}\right)}
\end{aligned}
$$

The open circuit potential can be derived by setting $I_{T}=0$ :

$$
V_{O C P}=\frac{-1}{\left(\alpha_{6}+\alpha_{9}\right) \cdot \gamma \cdot \alpha} \ln [c /(b-a)]
$$

The potential drop at the BOI, $\phi_{\mathrm{BOI}}$, is given by [1]

$$
\phi_{B O I}=\alpha \cdot V_{O C P}+\beta \cdot p H_{B O I}+\phi_{B O I}^{0}
$$

Figure 2-2 shows the postulated potential profile in the passive film on zirconium under PWR coolant conditions. Under open circuit conditions, the potential drop across the outer layer, $\Delta \phi_{o l}$, is 0 , and the potential drop across the barrier layer, $V_{b l}$, is given by,

$$
\overrightarrow{V_{O C P}}=\overrightarrow{\phi_{m}-\phi_{3}}=\left(\overrightarrow{\phi_{m}-\phi_{1}}\right)+\left(\overrightarrow{\phi_{1}-\phi_{2}}\right)+\left(\overrightarrow{\phi_{2}-\phi_{3}}\right)=\overrightarrow{\phi_{M B I}}+\vec{\varepsilon} L_{b l}+\overrightarrow{\phi_{B O I}}
$$




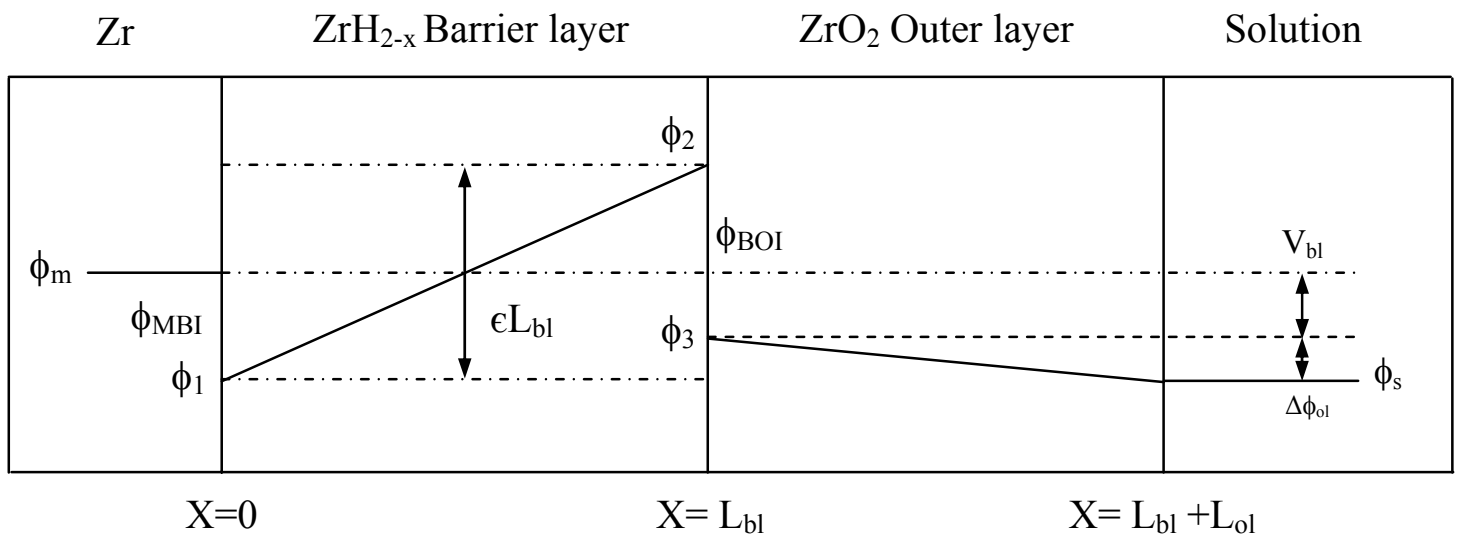

Figure 2-2. Postulated potential profile in the passive film on zirconium under PWR coolant conditions. Note that the diagram is not to scale and subscript "bl" designates "barrier layer", while "ol" signifies "outer layer".

Thus, we find that

$$
V_{O C P}=\phi_{M B I}-\varepsilon L_{b l}+\phi_{B O I}
$$

Substitution of Equations (2-4) and (2-7) into Equation (2-13) yields,

$$
\phi_{M B I}=\frac{1}{\alpha_{3} \cdot \gamma} \operatorname{In}\left(\frac{\theta \cdot a_{V_{H}^{*}}^{B O I} \cdot k_{6}^{0}}{2 k_{3}^{0}}\right)+\frac{\alpha_{6}}{\alpha_{3}} \phi_{B O I}
$$

Substitution of Equations (2-23) and (2-26) into Equation (2-25) yields the thickness of the hydride barrier layer under PWR coolant conditions.

$$
L_{b l}=\frac{1}{\alpha_{3} \cdot \gamma \cdot \varepsilon} \operatorname{In}\left(\frac{\theta \cdot a_{V_{H}^{B}}^{B O I} k_{6}^{0}}{2 k_{3}^{0}}\right)+\left(1+\frac{\alpha_{6}}{\alpha_{3}}\right) \frac{\left(\beta \cdot p H_{B O I}+\phi_{B O I}^{0}\right)}{\varepsilon}+\frac{1}{\varepsilon} \cdot\left[\left(\alpha-1+\frac{\alpha_{6}}{\alpha_{3}} \cdot \alpha\right) \cdot V_{O C P}\right]
$$

In the interior of the pore, the reaction $\mathrm{ZrO}^{2+}+\mathrm{H}_{2} \mathrm{O} \rightarrow \mathrm{ZrO}_{2}+2 \mathrm{H}^{+}$may be assumed to be in equilibrium, so the Gibbs energy change, $\Delta G_{T, P}$, should be 0 .

$$
\mu_{\mathrm{ZrO}_{2}}+2 \mu_{\mathrm{H}^{+}}-\mu_{\mathrm{ZrO} \mathrm{O}^{2+}}-\mu_{\mathrm{H}_{2} \mathrm{O}}=0
$$

We assume that the concentration of $\mathrm{ZrO}^{2+}$ ions inside the pores is not position dependent. This postulate is based on the concept that, because the pore diameter is small, the pore solution is in chemical equilibrium with a phase $\mathrm{ZrO}^{2+}$ whose composition is invariant with distance through the precipitated outer layer. The activity of $\mathrm{ZrO}^{2+}$ is defined as follows: 


$$
a_{\mathrm{ZrO}^{2+}}=\gamma_{\mathrm{ZrO} \mathrm{O}^{2+}} \cdot \mathrm{C}_{\mathrm{ZrO} \mathrm{O}^{2+}} / \mathrm{C}_{\mathrm{ZrO} \mathrm{O}^{2+}}^{0}
$$

where $C_{\mathrm{ZrO}^{2+}}^{0}$ is the standard-state concentration and $\gamma_{\mathrm{ZrO} \mathrm{O}^{2+}}$ is the activity coefficient of $\mathrm{ZrO}^{2+}$.

Zirconium cation vacancy and interstial, as defects, have low concentrations, so we assume that the $\mathrm{ZrO}^{2+}$ produced by Reactions (4) and (5) can be neglected.

$$
a_{Z r O^{2+}}=\tau_{0} \cdot e^{\alpha_{9} \cdot \gamma \cdot \alpha \cdot V_{O C P}}
$$

where the constant $\tau_{0}$ is defined in terms of the physical properties of the outer layer. According to Lewis' Equation,

$$
\begin{aligned}
& \mu_{\mathrm{ZrO}^{2+}}=\mu_{Z r O^{2+}}^{0}+R T \cdot \ln \left(\tau_{0} \cdot e^{\alpha_{9} \cdot \gamma \cdot \alpha \cdot V_{O C P}}\right) \\
& \mu_{H^{+}}=\mu_{H^{+}}^{0}+R T \cdot \ln \left(a_{H^{+}}^{\text {pore }}\right) \\
& \mu_{\mathrm{H}_{2} \mathrm{O}}=\mu_{\mathrm{H}_{2} \mathrm{O}}^{0}+R T \cdot \ln \left(a_{\mathrm{H}_{2} \mathrm{O}}^{\text {pore }}\right)
\end{aligned}
$$

where $\mu_{\mathrm{ZrO}^{2+}}^{o}, \mu_{\mathrm{H}_{2} \mathrm{O}}^{o}$ and $\mu_{\mathrm{H}^{+}}^{o}$ are the standard chemical potentials of $\mathrm{ZrO}^{2+}, \mathrm{H}_{2} \mathrm{O}$ and $\mathrm{H}^{+}$, respectively, in the interior of the pore. According to K.S. Pitzer [2], the Gibbs energy of the $\mathrm{ZrO}_{2}$ surface in a pore depends on the pore radius in the form:

$$
\mu_{\mathrm{ZrO}_{2}}=\mu_{\mathrm{ZrO}_{2}}^{0}+R T \cdot r_{0} / r
$$

where $r_{0}$ is a reference length; $r$ is the diameter of the pore; $\mu_{\mathrm{ZrO} 2}^{0}$ is the standard chemical potential of $\mathrm{ZrO}_{2}$ in a planar surface (i.e., for $r \rightarrow \infty$ ). Substitution of Equations (2-31) to (2-34) into Equation (2-28) yields

$$
r=\frac{r_{0}}{\left(\mu_{Z r O^{2+}}^{0}+\mu_{H_{2} O}^{0}-\mu_{Z r O_{2}}^{0}-2 \mu_{H^{+}}^{0}\right) / R T+\operatorname{In} \tau_{0}-2 \operatorname{Ina} H_{H^{+}}^{\text {pore }}+\alpha_{9} \gamma \cdot \alpha \cdot V_{O C P}}
$$

The porosity of the outer layer, $\theta$,

$$
\theta=P \cdot \pi \cdot r^{2}=\frac{P \cdot \pi \cdot r_{0}^{2}}{\left[\left(\mu_{Z r O^{+}}^{0}+\mu_{H_{2} O}^{0}-\mu_{Z r Q_{2}}^{0}-2 \mu_{H^{+}}^{0}\right) / R T+\operatorname{In} \tau_{0}-2 \operatorname{In} d_{H^{+}}^{\text {pore }}+\alpha_{9} \gamma \cdot \alpha \cdot V_{O C P}\right]^{2}}
$$

where $P$ is the number of pores per unit surface area. 


\section{BWR Coolant Conditions}

Following the same procedures of PWRs, we have the equations for total current density, partial anodic current density, partial cathodic current density, and open circuit potential for BWRs.

Under open circuit conditions, the total, partial anodic and cathodic steady-state current densities on the surface of the working electrode are as follows:

$$
\begin{aligned}
& \left.I_{T}=F \cdot \mid\left(4 k_{1}^{0} \cdot a_{V_{L_{r}}^{-}}^{M B I} / k_{3}^{0}+4 \mathrm{k}_{2}^{0} / \mathrm{k}_{3}^{0}+4\right) \cdot k_{3}^{0} \cdot e^{\alpha_{3} \cdot \cdot \cdot \phi_{M B I}}-4 \theta \cdot k_{7}^{0} C_{O_{2}}^{B O I}\left[a_{H^{+}}^{B O I}\right]^{n} \cdot e^{-\alpha_{7} \cdot \cdot \cdot \phi_{B O I}}\right] \\
& =F \cdot\left[\begin{array}{l}
\left(4 k_{1}^{0} \cdot a_{V_{L^{-}}^{4}}^{M B I} / k_{3}^{0}+4 k_{2}^{0} / k_{3}^{0}+4\right) \cdot k_{3}^{0} \cdot e^{\alpha_{3} \cdot \cdot\left[(1-\alpha) \cdot V_{O C P}-\varepsilon \cdot L_{b l}-\beta \cdot p H_{B O I}-\phi_{B O I}^{0}\right]} \\
-4 \theta \cdot k_{7}^{0} \cdot C_{O_{2}}^{B O I} \cdot\left[a_{H^{+}}^{B O I}\right]^{n} \cdot e^{-\alpha_{7} \cdot \cdot \cdot\left(\alpha \cdot V_{O C P}+\beta \cdot p H_{B O I}+\phi_{B O I}^{0}\right)}
\end{array}\right] \\
& =F \cdot\left(\hat{a} \cdot e^{\alpha_{3} \cdot \gamma \cdot(1-\alpha) \cdot V_{O C P}}-\hat{b} \cdot \theta \cdot e^{-\alpha_{7} \cdot \gamma \cdot \alpha \cdot V_{O C P}}\right) \\
& I_{a}=F \cdot \hat{a} \cdot e^{\alpha_{3} \cdot \gamma \cdot(1-\alpha) \cdot V_{O C P}} \\
& I_{c}=\left|I_{T}-I_{a}\right|=F \cdot \theta \cdot \hat{b} \cdot e^{-\alpha_{7} \cdot \gamma \cdot \alpha \cdot V_{O C P}}
\end{aligned}
$$

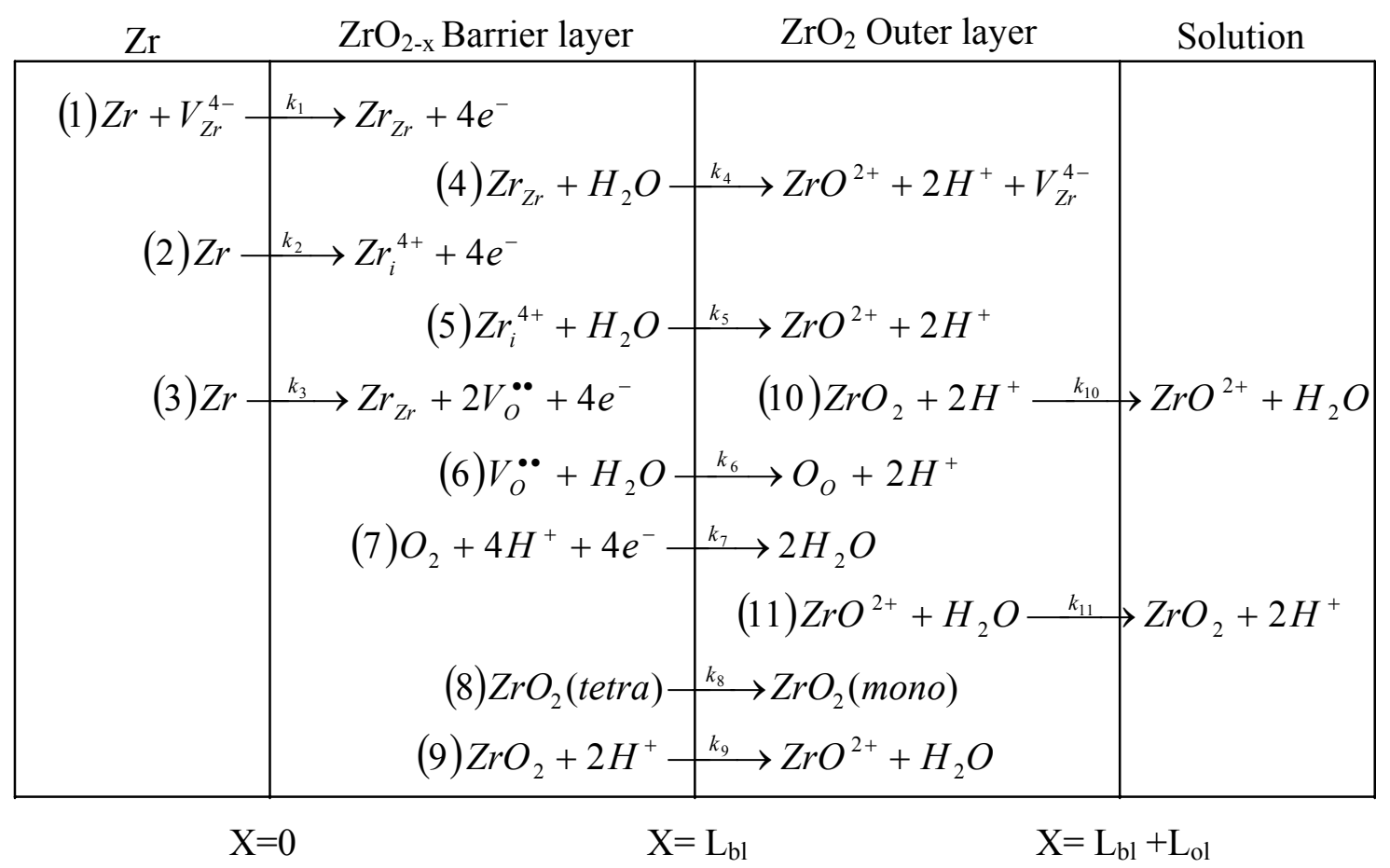

Figure 2-3. Interfacial reactions leading to generation and annihilation of point defects within the passive film on zirconium under BWR coolant conditions. $V_{O}^{\bullet \bullet}=$ Oxygen anion vacancy, $O_{O}=$ oxygen ion in a normal site on the oxygen sublattice. The other species are identified in the caption of Figure 2-1. 
where

$$
\begin{aligned}
& \hat{a}=\left(4 \frac{k_{1}^{0}}{k_{3}^{0}} \cdot a_{V_{Z r}^{4}}^{M B I}+4 \frac{k_{2}^{0}}{k_{3}^{0}}+4\right) \cdot k_{3}^{0} \cdot e^{\alpha_{3} \gamma\left(-\varepsilon \cdot L_{b l}-\beta \cdot p H_{B O I}-\phi_{B O I}^{0}\right)} \\
& \hat{b}=4 k_{7}^{0} \cdot\left(a_{H^{+}}^{B O I}\right)^{n} C_{O_{2}}^{B O I} \cdot e^{-\alpha_{7} \gamma\left(\beta \cdot p H_{B O I}+\phi_{B O I}^{0}\right)}
\end{aligned}
$$

The open circuit potential can be derived by setting $I_{T}=0$ to yield:

$$
V_{O C P}=\frac{1}{\left[\alpha_{3}(1-\alpha)+\alpha_{7} \cdot \alpha\right] \cdot \gamma} \ln (\hat{b} \cdot \theta / \hat{a})
$$

Figure 2-4 shows the postulated potential profile in the passive film on zirconium under BWR coolant conditions. The potential drop at the BOI, $\phi_{\mathrm{BOI}}$, is given by [1]

$$
\phi_{B O I}=\alpha \cdot V_{O C P}+\beta \cdot p H_{B O I}+\phi_{B O I}^{0}
$$

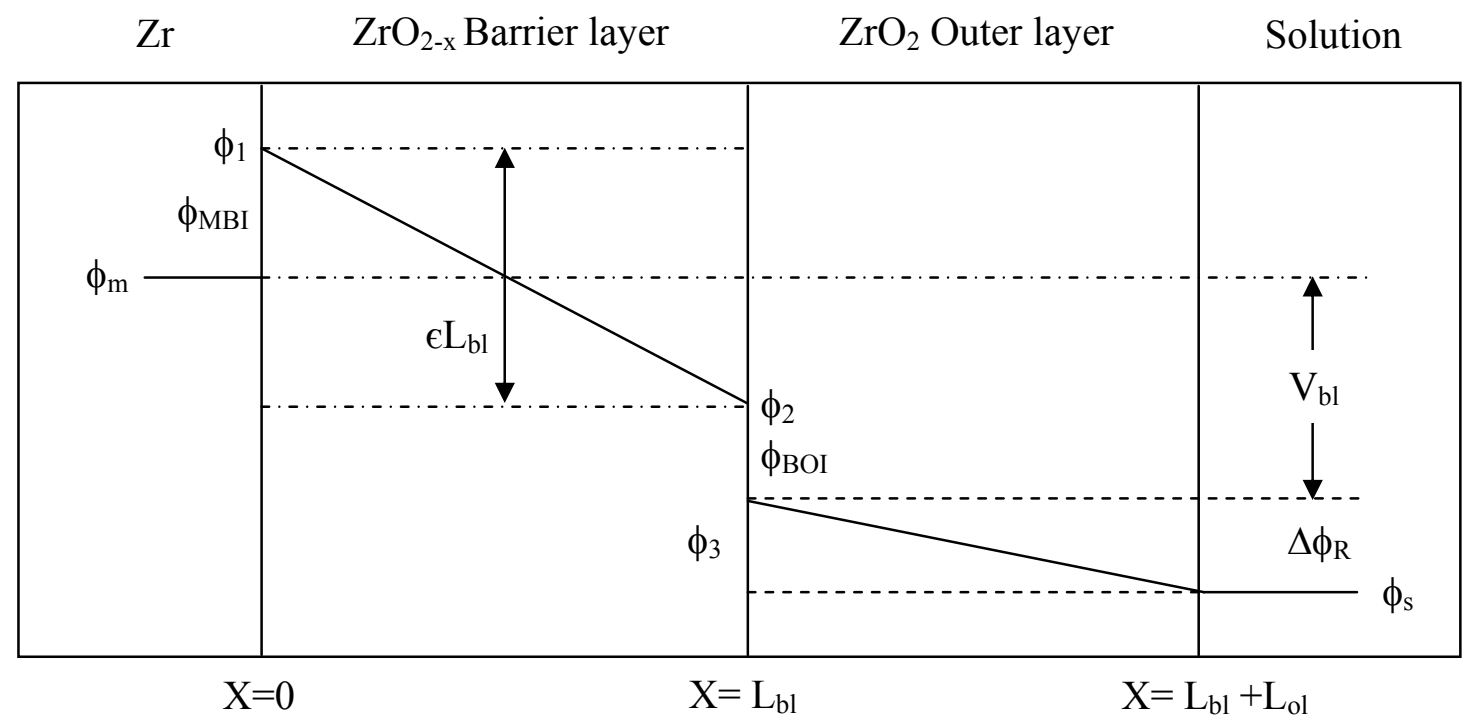

Figure 2-4. Postulated potential profile in the passive film on zirconium under BWR coolant conditions. Note that the diagram is not to scale and subscript "bl" designates "barrier layer", while "ol" signifies "outer layer".

From Figure 2-4, the open circuit potential is given by,

$$
\overrightarrow{V_{O C P}}=\overrightarrow{\phi_{m}-\phi_{3}}=\left(\overrightarrow{\phi_{m}-\phi_{1}}\right)+\left(\overrightarrow{\phi_{1}-\phi_{2}}\right)+\left(\overrightarrow{\phi_{2}-\phi_{3}}\right)=\overrightarrow{\phi_{M B I}}+\vec{\varepsilon} L_{b l}+\overrightarrow{\phi_{B O I}}
$$

Thus,

$$
V_{O C P}=\phi_{M B I}+\varepsilon L_{b l}+\phi_{B O I}
$$




$$
\phi_{M B I}=\frac{1}{\alpha_{3} \cdot \gamma} \operatorname{In}\left(\frac{\theta \cdot k_{6}^{0} \cdot a_{V_{0}^{*}}^{B O I}}{2 k_{3}^{0}}\right)+\frac{\alpha_{6}}{\alpha_{3}} \phi_{B O I}
$$

The thickness of the oxide barrier layer under open circuit conditions is as before to give:

$$
L_{b l}=\frac{-1}{\alpha_{3} \cdot \gamma \cdot \varepsilon} \operatorname{In}\left(\frac{\theta \cdot k_{6}^{0} a_{V_{0}^{*}}^{B O I}}{2 k_{3}^{0}}\right)-\left(1+\frac{\alpha_{6}}{\alpha_{3}}\right) \frac{\left(\beta \cdot p H_{B O I}+\phi_{B O I}^{0}\right)}{\varepsilon}-\frac{1}{\varepsilon} \cdot\left[\left(\alpha-1+\frac{\alpha_{6}}{\alpha_{3}} \alpha\right) \cdot V_{O C P}\right]
$$

The thickness and porosity of the BWR outer layer in the form of the equations are the same as those under PWR coolant conditions.

\subsection{Impedance Models}

\subsubsection{Barrier and Outer Layers Formed Under PWR Coolant Conditions}

Mott-Schottky analysis shows the film displays a n-type electronic character, indicating a preponderance of hydride vacancies and/or zirconium interstitials in the barrier layer, assuming the outer layer oxide is in a stoichiometric phase. Figure 2-5 displays the interfacial defect generation and annihilation reactions, which are envisioned to occur in the system together with barrier layer dissolution and outer layer formation and dissolution. Reaction (1) is responsible for the injection of the interstitial, $\mathrm{Zr}_{i}^{2+}$, into the barrier layer at the metal/barrier layer interface (MBI). The interstitial ion is then transmitted through the barrier layer and ejected into the outer layer as described by Reaction (3). Reaction (2) leads to the growth of the barrier layer into the metal substrate via the generation of hydride vacancies, and Reaction (5) results in the destruction of the barrier layer by dissolution. Reaction (4) describes hydride vacancy annihilation by hydride ion injection via the reduction of water (or possibly $\mathrm{H}^{+}$) at the barrier layer/outer layer interface (BOI). Note, Reactions (1), (3) \& (4) are lattice-conservative processes, as they don't result in the movement of the interface with respect to a laboratory frame of reference, whereas Reactions (2) \& (5) are non-conservative. A steady state must involve at least two non-conservative reactions, since only one non-conservative reaction would lead to monotonic growth or thinning of the passive film. Reactions (6) \& (7) correspond to the formation and dissolution of the outer layer. Note that Figure 2-5 is not scaled.

All interfacial reactions that produce or consume electrons contribute to the total steady-state current density, $I$.

$$
I=F \cdot\left[2 k_{1}+2 k_{2}+2 \theta \cdot k_{3} \cdot a_{Z r_{i}^{2+}}^{B O I}+6 \theta \cdot k_{5} \cdot\left(a_{O H^{-}}^{B O I}\right)^{m}-2 \theta \cdot k_{4} \cdot a_{V_{H}^{+}}^{B O I} \cdot a_{W}^{B O I}\right\rfloor
$$

where $\theta$ is the porosity of the outer layer; $a_{w}^{B O I}$ is the activity of water at the BOI; $a_{Z r_{i}^{2+}}^{B O I}$ and $a_{V_{H}}^{B O I}$ are the activities of the $Z r_{i}^{2+}$ and $V_{H}^{*}$ on the sublattice of the barrier layer at the BOI, 
respectively; and $\mathrm{m}$ is the kinetic order of the film dissolution reaction with respect to the activity of hydroxide ion.

\begin{tabular}{|c|c|c|c|}
\hline $\mathrm{Zr}$ & $\mathrm{ZrH}_{2-\mathrm{x}}$ Barrier layer & $\mathrm{ZrO}_{2}$ Outer layer & Solution \\
\hline \multirow[t]{2}{*}{$(1) Z r$} & $\stackrel{k_{1}}{\longrightarrow} Z r_{i}^{2+}+2 e^{-}$ & (6) $\mathrm{ZrO}^{2+}+\mathrm{H}_{2} \mathrm{O}$ & $\rightarrow \mathrm{ZrO}_{2}+2 \mathrm{H}^{+}$ \\
\hline & & $\stackrel{k_{3}}{\longrightarrow} \mathrm{ZrO}^{2+}+2 H^{+}+2 e^{-}$ & \\
\hline \multirow[t]{3}{*}{ (2) $Z r-$} & $\stackrel{k_{2}}{\longrightarrow} Z r_{Z r}+2 V_{H}^{\bullet}+2 e^{-}$ & (7) $\mathrm{ZrO}_{2}+2 \mathrm{H}^{+}$ & $\rightarrow \mathrm{ZrO}^{2+}+\mathrm{H}_{2} \mathrm{O}$ \\
\hline & $(4) V_{H}^{\bullet}+H_{2} \mathrm{O}+2 e^{-}$ & $\stackrel{k_{4}}{\longrightarrow} H_{H}+\mathrm{OH}^{-}$ & \\
\hline & $(5) \mathrm{ZrH}_{2}+4 \mathrm{OH}^{-}$ & $\stackrel{k_{5}}{\longrightarrow} \mathrm{ZrO}^{2+}+3 \mathrm{H}_{2} \mathrm{O}+6 e^{-}$ & \\
\hline
\end{tabular}

Figure 2-5. Interfacial reactions leading to the generation and annihilation of point defects within the barrier layer and to the formation of both the barrier layer and outer layer of the passive film on zirconium under PWR coolant conditions. $Z r=$ zirconium atom, $Z r_{Z r}=$ zirconium cation in a normal cation site on the metal sublattice in the barrier layer, $V_{H}^{\cdot}=$ hydrogen anion vacancy on the anion sublattice of the barrier layer, $H_{H}=$ hydrogen anion in an anion site on the anion sublattice of the barrier layer. The species are identified in the caption of Fig. 2-1.

The reaction rate constants, $k_{j}$, are functions of the potential drops across the two interfaces, and may be functions of the potential drops across the barrier and outer layers, and the barrier layer thickness, $L_{b l}$, and the $p H$ at the BOI. In any event, the potential applied at the BOI interface is related to the potential applied across the entire system, $V$, and the potential drop across the outer layer, $I \cdot R_{o l}$. The rate constants can then be written from the activated complex theory as:

$$
\begin{aligned}
& k_{1}=k_{1}^{0} \cdot e^{a_{1} \cdot\left(V-I \cdot R_{o l}\right)} \cdot e^{b_{1} \cdot L_{b l}} \cdot e^{c_{1} \cdot p H} \\
& k_{2}=k_{2}^{0} \cdot e^{a_{2} \cdot\left(V-I \cdot R_{o l}\right)} \cdot e^{b_{2} \cdot L_{b l}} \cdot e^{c_{2} \cdot p H} \\
& k_{3}=k_{3}^{0} \cdot e^{a_{3} \cdot\left(V-I \cdot R_{o l}\right)} \cdot e^{c_{3} \cdot p H} \\
& k_{4}=k_{4}^{0} \cdot e^{a_{4} \cdot\left(V-I \cdot R_{o l}\right)} \cdot e^{c_{4} \cdot p H} \\
& k_{5}=k_{5}^{0} \cdot e^{a_{5} \cdot\left(V-I \cdot R_{o l}\right)} \cdot e^{c_{5} \cdot p H}
\end{aligned}
$$

where $I$ and $R_{o l}$ are the current density $\left(\mathrm{A} / \mathrm{cm}^{2}\right)$ and the specific resistances $\left(\Omega \cdot \mathrm{cm}^{2}\right)$ of the porous outer layer, respectively.

The unknown rate constants for the interfacial reactions are shown in Table 2-1 where the reaction rate expressions take the form of $k_{i}=k_{i}^{0} \cdot e^{a_{i} \cdot\left(V-I \cdot R_{o l}\right)} \cdot e^{b_{i} L_{b l}} \cdot e^{c_{i} \cdot p H_{B O I}}$. Note, only the rate constants for the reactions that occur at the MBI [Reactions (1) and 
(2)] are functions of the thickness of the barrier layer, and all of the rate constants are functions of the applied voltage, $V$, and the voltage drop across the outer layer, $I \cdot R_{o l} . \quad \varepsilon$ is the electric field strength across the barrier layer, which is postulated to be a constant and independent of the applied voltage in the steady state, because of the buffering action of band-to-band (Esaki) or band-to-inter-bandgap state tunneling [3];

Table 2-1 Rate constants for interfacial reactions under PWR conditions

\begin{tabular}{|c|c|c|c|}
\hline Reaction & $a_{i}\left(\mathrm{~V}^{-1}\right)$ & $b_{i}\left(\mathrm{~cm}^{-1}\right)$ & $k_{i}^{0}(\mathrm{~cm} / \mathrm{s})$ \\
\hline (1) $Z r \stackrel{k_{1}}{\longrightarrow} Z r_{i}^{2+}+2 e^{-}$ & $2 \alpha_{1}(1-\alpha) \gamma$ & $-2 \alpha_{1} \gamma \cdot \varepsilon$ & $k_{1}^{00} e^{-2 \alpha_{1} \cdot \gamma \cdot\left(\phi_{B O I}^{o}+\beta \cdot P H\right)}$ \\
\hline$(2) Z r \stackrel{k_{2}}{\longrightarrow} Z r_{Z r}+2 V_{H}^{\bullet}+2 e^{-}$ & $2 \alpha_{2}(1-\alpha) \gamma$ & $-2 \alpha_{2} \gamma \cdot \varepsilon$ & $k_{2}^{00} e^{-2 \alpha 2 \cdot \gamma \cdot\left(\phi_{B O I}^{o}+\beta \cdot P H\right)}$ \\
\hline (3) $\mathrm{Zr}_{i}^{2+}+\mathrm{H}_{2} \mathrm{O} \stackrel{k_{3}}{\longrightarrow} \mathrm{ZrO}^{2+}+2 \mathrm{H}^{+}+2 e^{-}$ & $4 \alpha_{3} \alpha \cdot \gamma$ & 0 & $k_{3}^{00} e^{4 \alpha_{3} \cdot \gamma \cdot\left(\phi_{B O I}^{o}+\beta \cdot P H\right)}$ \\
\hline$(4) V_{H}^{\bullet}+H_{2} \mathrm{O}+2 e^{-} \stackrel{k_{4}}{\longrightarrow} H_{H}+O H^{-}$ & $-\alpha_{4} \alpha \cdot \gamma$ & 0 & $\left.k{ }_{4}^{00} e^{-\alpha_{4} \cdot \gamma \cdot\left(\phi_{B O I}^{o}+\beta \cdot P H\right.}\right)$ \\
\hline (5) $\mathrm{ZrH}_{2}+4 \mathrm{OH}^{-} \stackrel{k_{5}}{\longrightarrow} \mathrm{ZrO}^{2+}+3 \mathrm{H}_{2} \mathrm{O}+6 e^{-}$ & $6 \alpha_{5} \alpha \cdot \gamma$ & 0 & $k_{5}^{00} e^{6 \alpha_{5} \cdot \gamma \cdot\left(\phi_{B O I}^{o}+\beta \cdot P H\right)}$ \\
\hline
\end{tabular}

where $\gamma=F / R T ; \alpha=d \Phi_{B O I} / d V$, and $\beta=d \Phi_{B O I} / d p H$.

In order to simplify the model, the $p H$ at the BOI is assumed to be a constant, not a variable, and hereinafter $e^{c_{i} \cdot p H}$ is merged into $k_{i}^{0}$. The steady-state constraints are invoked to construct an impedance expression that is consistent with the zero frequency limit. For example, requiring that the generation rate of interstitial ions or hydrogen vacancies at the MBI [Reaction (1) or (2) in Fig. 2-5] is equal to the annihilation rate of the same species at the BOI [Reaction (3) or (4) in Fig. 2-5] yields:

$$
\begin{aligned}
& k_{1}=\theta \cdot a_{Z r_{i}^{r^{+}}}^{B O I} \cdot a_{W}^{B O I} \cdot k_{3} \\
& 2 k_{2} \approx \theta \cdot a_{V_{H}^{B}}^{B O I} \cdot a_{W}^{B O I} \cdot k_{4}
\end{aligned}
$$

Equations (2-52) and (2-53) are valid at steady state, and assumed to be approximately valid under non-steady-state conditions. The solution in the pore interior is assumed to be dilute, so that $a_{W}^{B O I}$ is taken to be 1. Substitution of Equations (2-52) and (2-53) into Equation (2-46) yields:

$$
I \approx F \cdot\left[4 k_{1}-2 k_{2}+6 \theta \cdot k_{5} \cdot\left(a_{O H^{-}}^{B O I}\right)^{m}\right]
$$

Note that the current density is a function of $V$ and $L_{b l}$. Accordingly, for any arbitrary changes $\delta V$ and $\delta L_{b l}$, we can derive the following expressions by assuming that $\theta$ is constant: 


$$
\begin{aligned}
& \delta I=F \cdot\left[4 \delta k_{1}-2 \delta k_{2}+6 \theta \cdot \delta k_{5}\left(a_{O H^{-}}^{B O I}\right)^{m}\right] \\
& \delta k_{1}=a_{1} k_{1} \delta V+b_{1} k_{1} \delta L_{b l}-a_{1} k_{1} R_{o l} \delta I \\
& \delta k_{2}=a_{2} k_{2} \delta V+b_{2} k_{2} \delta L_{b l}-a_{2} k_{2} R_{o l} \delta I
\end{aligned}
$$

and

$$
\delta k_{5}=a_{5} k_{5} \delta V-a_{5} k_{5} R_{o l} \delta I
$$

Substitution of Equations (2-56) to (2-58) into Equation (2-55) yields:

$$
\delta I=F\left[\begin{array}{l}
\left(4 a_{1} k_{1}-2 a_{2} k_{2}+6 \theta \cdot a_{5} k_{5}\left(a_{O H^{-}}^{B O I}\right)^{m}\right) \delta V+\left(4 b_{1} k_{1}-2 b_{2} k_{2}\right) \delta L_{b l} \\
+R_{o l}\left[-4 a_{1} k_{1}+2 a_{2} k_{2}-6 \theta \cdot\left(a_{O H^{-}}^{B O I}\right)^{m} a_{5} k_{5}\right] \delta I
\end{array}\right]
$$

The variations in $I, V$ and $L_{b l}$ are sinusoidal in an EIS measurement, so that we can write:

$$
\begin{aligned}
& \delta I=\Delta I \cdot e^{j \cdot \omega \cdot t} \\
& \delta V=\Delta V \cdot e^{j \cdot \omega \cdot t} \\
& \delta L_{b l}=\Delta L_{b l} \cdot e^{j \cdot \omega \cdot t} \\
& C_{O H^{-}}^{B O I}=\frac{10^{-p K_{w}(T)-6}}{C_{H^{+}}^{B O I}} \quad \text { (for the unit } \mathrm{cm}^{3} \text { ) }
\end{aligned}
$$

where $K_{w}(T)$ is the water dissociation constant, which is a function of temperature [4]. Substitution of Equations (2-60) to (2-63) into Equation (2-59) divided by $\delta V$ yields the Faradic admittance, $Y_{f}$, as follows:

$$
Y_{f}=\frac{F \cdot S_{1}}{S_{0}}+\frac{F\left(4 b_{1} k_{1}-2 b_{2} k_{2}\right)}{S_{0}} \frac{\Delta L_{b l}}{\Delta V}
$$

where

$$
\begin{aligned}
& S_{0}=1+F R_{o l}\left[4 a_{1} k_{1}-2 a_{2} k_{2}+6 \theta \cdot a_{5} k_{5}\left(10^{-p K_{w}(T)-6} / C_{H^{+}}^{B O I}\right)^{m}\right] \\
& S_{1}=4 a_{1} k_{1}-2 a_{2} k_{2}+6 \theta \cdot a_{5} k_{5}\left(10^{-p K_{w}(T)-6} / C_{H^{+}}^{B O I}\right)^{m}
\end{aligned}
$$

The derivation for $\Delta L_{b l} / \Delta V$ is as follows:

$$
\frac{d L_{b l}}{d t}=\Omega \cdot k_{2}-\Omega \cdot \theta \cdot k_{5}\left(\frac{10^{-p K_{w}(T)-6}}{C_{H^{+}}^{B O I}}\right)^{m}
$$

where $\Omega=17.04 \mathrm{cc} / \mathrm{mol}$ is the volume per mole of cation of the barrier layer. Thus, 


$$
\begin{aligned}
\frac{d \delta L_{b l}}{d t} & =j \omega \cdot \Delta L_{b l} \cdot e^{j \cdot \omega t} \\
& =\Omega \cdot\left[k_{2} a_{2}-\theta \cdot k_{5} a_{5}\left(\frac{10^{-p K_{w}(T)-6}}{C_{H^{+}}^{B O I}}\right)^{m}\right] \cdot \Delta V \cdot e^{j \cdot \omega t}+\Omega \cdot k_{2} b_{2} \cdot \Delta L_{b l} \cdot e^{j \cdot \omega t} \\
& -\Omega R_{o l}\left[k_{2} a_{2}-\theta \cdot k_{5} a_{5}\left(\frac{10^{-p K_{w}(T)-6}}{C_{H^{+}}^{B O I}}\right)^{m}\right] \cdot \Delta V \cdot e^{j \cdot \omega t}
\end{aligned}
$$

Substitution of Equations (2-60) to (2-62), followed by rearrangement, yields

$$
\frac{\Delta L_{b l}}{\Delta V}=\frac{A_{1}}{j \omega-\Omega \cdot k_{2} b_{2}}-\frac{A_{1} R_{o l}}{j \omega-\Omega \cdot k_{2} b_{2}} \cdot Y_{f}
$$

where $A_{1}=\Omega \cdot\left[k_{2} a_{2}-\theta \cdot k_{5} a_{5}\left(\frac{10^{-p K_{w}(T)-6}}{C_{H^{+}}^{B O I}}\right)^{m}\right]$. Thus substitution of Equation (2-67) into Equation (2-64) yields the Faradic admittance, $Y_{f}$, under PWR coolant conditions as:

$$
Y_{f}=\frac{F\left(4 k_{1} b_{1}-2 k_{2} b_{2}\right) A_{1}+F S_{1}\left(j \omega-\Omega b_{2} k_{2}\right)}{F A_{1} R_{o l}\left(4 b_{1} k_{1}-2 b_{2} k_{2}\right)+S_{0}\left(j \omega-\Omega b_{2} k_{2}\right)}
$$

The total impedance comprises the impedance of the barrier layer, including those of the two interfaces, $Z_{b l}$, and that of the barrier layer itself, in series with that of the outer layer and the resistance of the bulk solution of the experimental system, $Z_{o l}$ and $R_{b s}$, respectively, as shown in Figure 2-6. As for the barrier layer, its impedance is the sum of four components: (a) impedance due to the Faradic process, $Z_{f}$ (i.e. $1 / Y_{f}$ ), (b) impedance due to geometric capacitance, $Z_{C_{b l}}$, (c) resistance due to the transport of electrons and the diffusion of electron holes, $Z_{e, h}$, and (d) the Warburg impedance, $Z_{w}$, due to the transport of defects within the layer. The latter is added in a somewhat ad hoc fashion, as it does

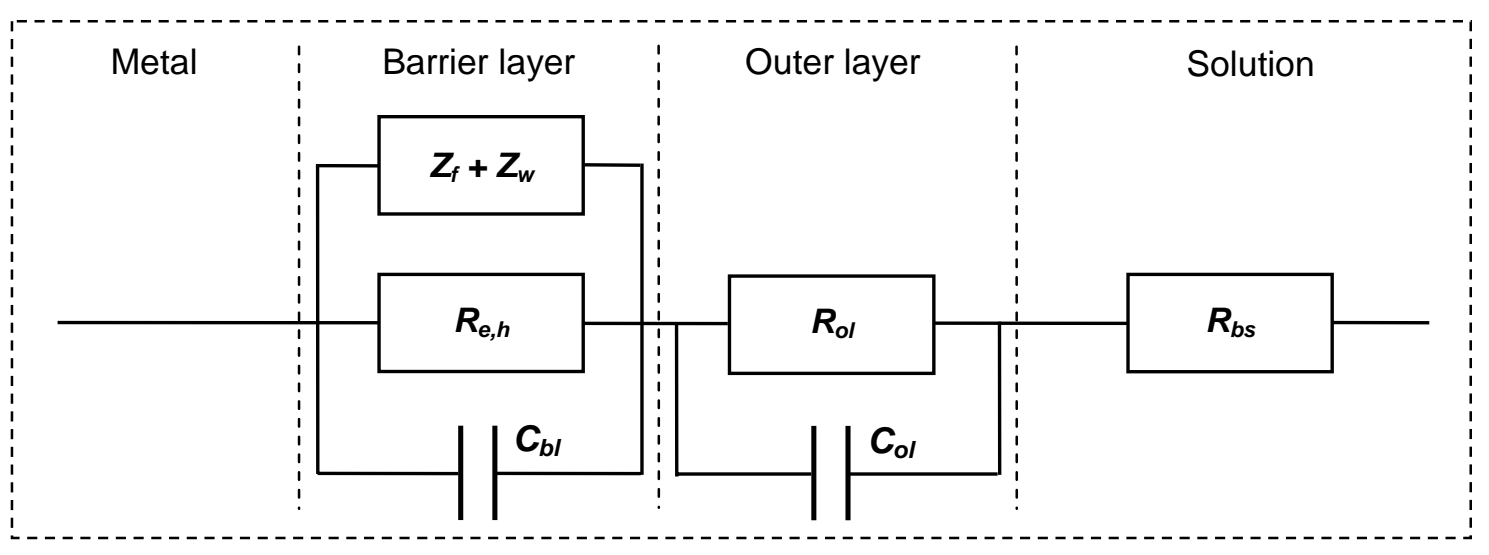

Figure 2-6. Equivalent circuit for the total impedance 
not strictly follow the model if interfacial reactions are assumed to be irreversible. However, as shown by Chao, Lin, and Macdonald [5] and later by Macdonald and Smedley [6], specification of reversible point defect generation and annihilation reactions at the interfaces does result in the presence of the Warburg term in the faradaic impedance. We, therefore, include the series Warburg term in the present model by assuming virtual reversibility of the interfacial reactions. As for the outer layer, its impedance comprises the resistance, $R_{o l}$, due to electrolyte in the pores in parallel with an impedance due to the geometric capacitance, $Z_{C_{o l}}$.

$$
Z_{\text {total }}=Z_{b l}+Z_{o l}+R_{b s}=\frac{1}{Z_{C_{b l}}^{-1}+\left(Z_{f}+Z_{w}\right)^{-1}+Z_{e, h}^{-1}}+\frac{1}{R_{o l}^{-1}+Z_{C_{o l}}^{-1}}+R_{b s}
$$

where $R_{o l}=\rho \cdot L_{o l} / \theta$, and $\rho$ is the resistivity of the solution in the outer layer pores.

\subsubsection{Barrier and Outer Layers Formed Under BWR Coolant Conditions}

Mott-Schottky analysis shows the film displays a n-type electronic character, indicating a preponderance of oxygen anion vacancies and/or zirconium interstitials in the barrier layer, assuming the outer layer oxide is in a stoichiometric phase. Figure 2-7 displays the interfacial defect generation and annihilation reactions envisioned to occur in the system together with barrier layer dissolution and outer layer formation and dissolution. Reaction (1) is responsible for the injection of the interstitial, $\mathrm{Zr}_{i}^{2+}$, into the barrier layer at the metal/barrier layer interface (MBI). The interstitial ion is then transmitted through the barrier layer and ejected into the outer layer as described by Reaction (3). Reaction (2) leads to the growth of the barrier layer into the metal substrate via the generation of oxygen vacancies, and Reaction (5) results in the destruction of the barrier layer by dissolution. Reaction (4) describes oxygen vacancy annihilation by oxygen ion injection via the reduction of water at the barrier layer/outer layer interface (BOI). Note, Reactions (1), (3) \& (4) are lattice-conservative processes, as they don't result in the movement of the interface with respect to a lab frame of reference, whereas Reactions (2) \& (5) are non-conservative. A steady state must involve at least two nonconservative reactions, since only one non-conservative reaction would lead to monotonic growth or thinning of the passive film. Reactions (6) \& (7) correspond to the formation and dissolution of the outer layer. Note that Figure 2-7 is not scaled.

All interfacial reactions that produce or consume electrons contribute to the total current density under closed circuit conditions, $I$.

$$
I=4 F \cdot\left[k_{1}+k_{2}\right]
$$

The reaction rate constants, $k_{j}$, are functions of the potential drops across the two interfaces, and may be functions of the potential drops across the barrier and outer layers and the barrier layer thickness, $L_{b l}$, and the $p H$ at the BOI. In any event, the potential applied at the BOI interface is related to the potential applied across the entire system, $V$, 
and the potential drop across the outer layer, $I \cdot R_{o l}$. The rate constants can then be written from the activated complex theory as:

$$
\begin{aligned}
& k_{1}=k_{1}^{0} \cdot e^{a_{1} \cdot\left(V-I \cdot R_{o l}\right)} \cdot e^{b_{1} \cdot L_{b l}} \cdot e^{c_{1} \cdot p H} \\
& k_{2}=k_{2}^{0} \cdot e^{a_{2} \cdot\left(V-I \cdot R_{o l}\right)} \cdot e^{b_{2} \cdot L_{b l}} \cdot e^{c_{2} \cdot p H} \\
& k_{3}=k_{3}^{0} \cdot e^{a_{3} \cdot\left(V-I \cdot R_{o l}\right)} \cdot e^{c_{3} \cdot p H} \\
& k_{4}=k_{4}^{0} \cdot e^{a_{4} \cdot\left(V-I \cdot R_{o l}\right)} \cdot e^{c_{4} \cdot p H} \\
& k_{5}=k_{5}^{0} \cdot e^{c_{5} \cdot p H}
\end{aligned}
$$

where $I$ and $R_{o l}$ are the current density and the specific resistances $\left(\Omega \cdot \mathrm{cm}^{2}\right)$ of the porous outer layer, respectively.

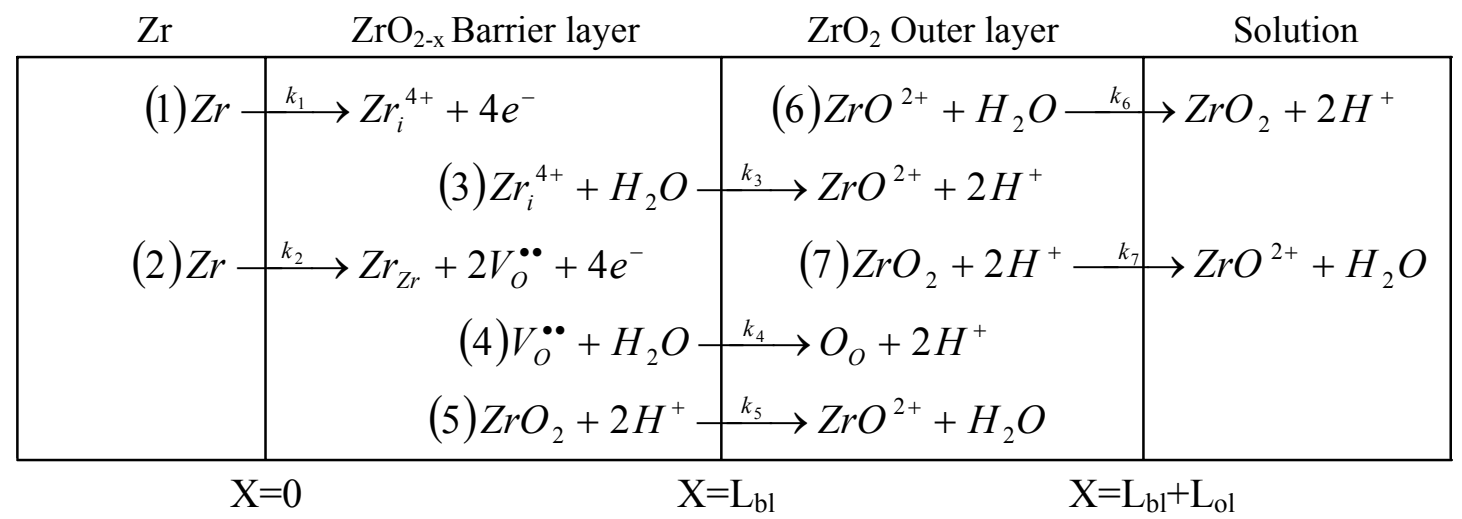

Figure 2-7. Primary interfacial reactions leading to the generation and annihilation of point defects within the barrier layer and to the formation of both the barrier layer and outer layer of the passive film on zirconium under BWR coolant conditions. The species are identified in the caption of Fig. 2-3.

The unknown rate constants for the interfacial reactions are shown in Table 2-2, where the reaction rate expressions take the form of $k_{i}=k_{i}^{0} \cdot e^{a_{i} \cdot\left(V-I \cdot R_{o l}\right)} \cdot e^{b_{i} L_{b l}} \cdot e^{c_{i} \cdot p H_{B O I}}$. Note, only the rate constants for the reactions that occur at the MBI [Reactions (1) and (2)] are functions of the thickness of the barrier layer, and all of the rate constants are functions of the applied voltage, $V$, and the voltage drop across the outer layer, $I \cdot R_{o l}$.

In order to simplify the model further and make the data fitting more feasible, we take the $p H$ at BOI to be a constant, not a variable, and hereinafter merge $e^{c_{i} \cdot p H}$ into $k_{i}^{0}$, and that the current due to cation interstitials (Reaction (1)) and other ions (such as $\mathrm{Fe}^{3+}$ ) is a small part of that due to oxygen vacancies (Reaction (2)) with a ratio of $x$, as the cation interstitials and other ions existing in the barrier layer are often in the absolute minority. Note that the total current density is a function of $V$, and $L_{b l}$. Accordingly, for any arbitrary changes $\delta V$, and $\delta L_{b l}$, we can derive as follows by assuming the $\mathrm{pH}$ at the 
BOI, the porosity of the outer layer, $\theta$, and the concentration of molecular oxygen, $C_{O_{2}}^{B O I}$, are constant, and the solution in the pore interior is dilute and the activity of the water at the BOI is taken to be 1 .

$$
\begin{aligned}
& \delta I=4 F(1+x) \cdot \delta k_{2} \\
& \delta k_{1}=a_{1} k_{1} \delta V+b_{1} k_{1} \delta L_{b l}-a_{1} k_{1} R_{o l} \delta I \\
& \delta k_{2}=a_{2} k_{2} \delta V+b_{2} k_{2} \delta L_{b l}-a_{2} k_{2} R_{o l} \delta I \\
& \delta k_{3}=a_{3} k_{3} \delta V-a_{3} k_{3} R_{o l} \delta I \\
& \delta k_{4}=a_{4} k_{4} \delta V-a_{4} k_{4} R_{o l} \delta I \\
& \delta k_{5}=0
\end{aligned}
$$

\begin{tabular}{|c|c|c|c|}
\hline Reaction & $a_{i}\left(\mathrm{~V}^{-1}\right)$ & $b_{i}\left(\mathrm{~cm}^{-1}\right)$ & $k_{i}^{0}(\mathrm{~cm} / \mathrm{s})$ \\
\hline$(1) Z r \stackrel{k_{1}}{\longrightarrow} Z r_{i}^{4+}+4 e^{-}$ & $4 \alpha_{1}(1-\alpha) \gamma$ & $-4 \alpha_{1} \gamma \cdot \varepsilon$ & $k_{1}^{00} e^{-4 \alpha_{1} \cdot \gamma \cdot\left(\phi_{B O I}^{o}+\beta \cdot P H\right)}$ \\
\hline (2) $Z r \stackrel{k_{2}}{\longrightarrow} Z r_{Z r}+2 V_{O}^{\bullet \bullet}+4 e^{-}$ & $4 \alpha_{2}(1-\alpha) \gamma$ & $-4 \alpha_{2} \gamma \cdot \varepsilon$ & $k_{2}^{00} e^{-4 \alpha_{2} \cdot \gamma \cdot\left(\phi_{B O I}^{O}+\beta \cdot P H\right)}$ \\
\hline (3) $\mathrm{Zr}_{i}^{4+}+\mathrm{H}_{2} \mathrm{O} \stackrel{k_{3}}{\longrightarrow} \mathrm{ZrO}^{2+}+2 \mathrm{H}^{+}$ & $4 \alpha_{3} \alpha \cdot \gamma$ & 0 & $k_{3}^{00} e^{4 \alpha_{3} \cdot \gamma \cdot\left(\phi_{B O I}^{o}+\beta \cdot P H\right)}$ \\
\hline (4) $V_{O}^{\bullet \bullet}+H_{2} O \stackrel{k_{4}}{\longrightarrow} O_{O}+2 H^{+}$ & $2 \alpha_{4} \alpha \cdot \gamma$ & 0 & $k_{4}^{00} e^{2 \alpha_{4} \cdot \gamma \cdot\left(\phi_{B O I}^{o}+\beta \cdot P H\right)}$ \\
\hline (5) $\mathrm{ZrO}_{2}+2 \mathrm{H}^{+} \stackrel{k_{5}}{\longrightarrow} \mathrm{ZrO}^{2+}+\mathrm{H}_{2} \mathrm{O}$ & 0 & 0 & $k_{5}^{o}$ \\
\hline
\end{tabular}

Table 2-2 Rate constants for interfacial reactions under BWR conditions

where $\gamma=F / R T ; \alpha=d \Phi_{B O I} / d V$, and $\beta=d \Phi_{B O I} / d p H$.

Substitution of Equations (2-77) and (2-78) into Equation (2-76) yields:

$$
\left.\delta I=4 F(1+x)\left(a_{2} k_{2} \delta V+b_{2} k_{2} \delta L_{b l}-a_{2} k_{2} R_{o l} \delta I\right]\right)
$$

The variations in the $I, V$, and $L_{b l}$ are sinusoidal in EIS measurement, so that we can write:

$$
\begin{aligned}
& \delta I=\Delta I \cdot e^{j \cdot \omega \cdot t} \\
& \delta V=\Delta V \cdot e^{j \cdot \omega \cdot t} \\
& \delta L_{b l}=\Delta L_{b l} \cdot e^{j \cdot \omega \cdot t}
\end{aligned}
$$


Substitution of Equations (2-83) to (2-85) into Equation (2-82) divided by $\delta V$ yields the Faradic admittance $Y_{f}$ as follows:

$$
Y_{f}=\frac{4 F(1+x) a_{2} k_{2}}{S_{0}}+\frac{4 F(1+x) b_{2} k_{2}}{S_{0}} \frac{\Delta L_{b l}}{\Delta V}
$$

where $S_{0}=1+4 F(1+x) a_{2} k_{2} R_{o l}$

The derivation for $\Delta L_{b l} / \Delta V$ is as follows:

$$
d L_{b l} / d t=\Omega \cdot k_{2}-\Omega \cdot k_{5}\left(C_{H^{+}}^{B O I}\right)^{n}
$$

where $\Omega=21.06 \mathrm{cc} / \mathrm{mol}$ is the volume per mole of cations in the barrier layer and $n$ is the kinetic order of the film dissolution with respect to the concentration of hydrogen ion. Taking the total differential as:

$$
\begin{aligned}
\frac{d \delta L_{b l}}{d t} & =j \omega \cdot \Delta_{b l} \cdot e^{j \cdot \omega t} \\
& =\Omega \cdot\left(a_{2} k_{2} \cdot \delta V+b_{2} k_{2} \cdot \delta L_{b l}-a_{2} k_{2} R_{o l} \delta\right)
\end{aligned}
$$

Substitution of Equations (2-83) to (2-85) into Equation (2-88), followed by rearrangement, yields

$$
\frac{\Delta L_{b l}}{\Delta V}=\frac{\Omega \cdot a_{2} k_{2}}{j \omega-\Omega \cdot b_{2} k_{2}}-\frac{\Omega \cdot a_{2} k_{2} R_{o l}}{j \omega-\Omega \cdot b_{2} k_{2}} \cdot Y_{f}
$$

Substitution of Equation (2-89) into Equation (2-86) yields the Faradic admittance $Y_{f}$ :

$$
Y_{f}=\frac{4 F \Omega \cdot a_{2} k_{2}(1+x) b_{2} k_{2}+4 F(1+x) a_{2} k_{2}\left(j \omega-\Omega b_{2} k_{2}\right)}{4 F \Omega \cdot a_{2} k_{2} R_{o l}(1+x) \cdot b_{2} k_{2}+S_{0}\left(j \omega-\Omega b_{2} k_{2}\right)}
$$

The total impedance comprises the impedance of the barrier layer, including those of the two interfaces, $Z_{b l}$, and that of the barrier layer itself, in series with that of the outer layer and the resistance of the bulk solution of the experimental system, $Z_{o l}$ and $R_{b s}$, respectively, as shown in Figure 2-6. As for the barrier layer, its impedance is the sum of four components: (a) impedance due to the Faradic process, $Z_{f}$ (i.e. $1 / Y_{f}$ ), (b) impedance due to geometric capacitance, $Z_{C_{b l}}$, (c) resistance due to the transport of electrons and the diffusion of electron holes, $Z_{e, h}$, and (d) the Warburg impedance, $Z_{w}$, due to the transport of defects within the layer. The latter is added in a somewhat ad hoc fashion, as it does not strictly follow from the model if the interfacial reactions are assumed to be irreversible. However, as shown by Chao, Lin, and Macdonald [5] and later by Macdonald and Smedley [6], specification of reversible point defect generation and annihilation reactions at the interfaces does result in the presence of the Warburg term in the faradaic impedance. Furthermore, there is such a feature in the experimental data. Therefore, we include the series Warburg term in the present model by assuming virtual reversibility of the interfacial reactions. As for the outer layer, its impedance comprises 
the resistance, $R_{o l}$, due to electrolyte in the pores in parallel with an impedance due to the geometric capacitance, $Z_{C_{o l}}$.

$$
Z_{\text {total }}=Z_{b l}+Z_{o l}+R_{b s}=\frac{1}{Z_{C_{b l}}^{-1}+\left(Z_{f}+Z_{w}\right)^{-1}+Z_{e, h}^{-1}}+\frac{1}{R_{o l}^{-1}+Z_{C_{o l}}^{-1}}+R_{b s}
$$

where $R_{o l}=\rho \cdot L_{o l} / \theta$, and $\rho$ is the resistivity of the solution in the outer layer pores.

\subsubsection{Validity of the Measured Impedance Data}

Electrochemical impedance spectra (EIS) for passive zirconium films were measured over a wide range of frequencies (typically $100 \mathrm{kHz}$ to $0.01 \mathrm{~Hz}$ ) as a function of formation potential across the passive range in $0.1 \mathrm{M} \mathrm{B}(\mathrm{OH})_{3}+0.001 \mathrm{M} \mathrm{LiOH}$ solutions $\left(\mathrm{pH}=6.94\right.$ at $\left.250^{\circ} \mathrm{C}\right)$. The impedance was measured after holding the potential constant at each point for 24 hours, in order to ensure that the passive film exists in the steady state. The measurements were carried out in the ascending potential direction. Since the stability of the electrochemical system during EIS measurement is critical to obtaining viable data, the quality of the EIS data was checked both experimentally and theoretically. The data were checked experimentally by stepping the frequencies from high-to-low and then immediately back from low-to-high, with the impedance being measured at each step, to ascertain that the same values were obtained at equivalent frequencies in the two directions. If the system is in the steady state, which means the thickness and current are independent of time, the impedance data should match in the two frequency step directions. Coincidence is observed in all impedance measurements for passive films formed at different potentials. This is a particularly good test for system stability.

The quality of the impedance data was also checked using the Kramers-Kronig transforms. These integral transforms test for compliance of the system with the linearity, stability, and causality constraints of Linear Systems Theory (LST). The K-K transforms, which arise from Cauchy's theorem and the definition of causality, were first developed for the field of optics by establishing a connection between the real and imaginary parts of complex optical parameters (e.g., refractive index). The K-K transforms are purely mathematical results and do not reflect any assumptions concerning the physical properties of the system. Thus, only the experimental data satisfying the linearity, stability, and causality constraints, as indicated by the K-K transforms, can be used to describe the properties of the systems in terms of linearized model. Macdonald and Urquidi-Macdonald [7-11] developed methods for transforming the real part of the electrochemical impedance into the imaginary part of the impedance and vice versa. Their studies show that the K-K transforms are strongly sensitive to the violation of the stability constraint, but are not particularly sensitive to violation of the linearity condition. The K-K transforms for the impedance are basically a set of mathematical relations transforming the real part of impedance into the imaginary part of impedance and vice versa, stated as:

$$
\begin{aligned}
& Z^{\prime \prime} \rightarrow Z^{\prime}: \\
& Z^{\prime}(\omega)-Z^{\prime}(\infty)=\left(\frac{2}{\pi}\right) \int_{0}^{\infty} \frac{x Z^{\prime \prime}(x)-\omega Z^{\prime \prime}(\omega)}{x^{2}-\omega^{2}} d x
\end{aligned}
$$




$$
\begin{aligned}
& Z^{\prime}(\omega)-Z^{\prime}(0)=\left(\frac{2 \omega}{\pi}\right) \int_{0}^{\infty}\left[\left(\frac{\omega}{x}\right) Z^{\prime \prime}(x)-Z^{\prime \prime}(\omega)\right] \cdot \frac{1}{x^{2}-\omega^{2}} d x \\
& Z^{\prime} \rightarrow Z^{\prime \prime}: \\
& Z^{\prime \prime}(\omega)=-\left(\frac{2 \omega}{\pi}\right) \int_{0}^{\infty} \frac{Z^{\prime}(x)-Z^{\prime}(\omega)}{x^{2}-\omega^{2}} d x
\end{aligned}
$$

in which $Z^{\prime}$ and $Z^{\prime \prime}$ are the real and imaginary parts of the impedance, and $\omega$ and $x$ are frequencies.

\subsubsection{Extraction of Model Parameter Values from EIS Data}

The development of deterministic models for predicting the accumulation of corrosion damage to zirconium and Zircaloys in PWR and BWR coolant environments requires the acquisition of values for various model parameters. The DataFit (Version 8.0, www.curvefitting.com) commercial software was used in this work for optimization so as to obtain values for the standard rate constants, $k_{i}^{00}$, and transfer coefficients, $\alpha_{i}$, for the $i$ elementary interfacial reactions, the polarizability of the barrier layer/outer layer interface, $\alpha$, the electric field strength across barrier layer, $\varepsilon$, and other parameters as described below. Optimization was carried out in five stages as shown in Figure 2-8. First of all, the total impedance model was decomposed into two parts for real and imaginary components; secondly, the optimization model, together with the experimental impedance data upon which it will be optimized, needs to be carefully defined; thirdly, approximate parameter values are obtained by "manually optimizing" the model for $Z(\omega)$ on the experimental data; fourthly, optimize the parameter values using the Datafit code; and finally, test the optimization results. The optimization procedure ends if the result satisfies the following requirements simultaneously; if not, the procedure returns to the third stage and the model is optimized once again. These requirements include: (1) all the parameter values are physically reasonable and should exist within known bounds; (2) the calculated $Z^{\prime}(\omega)$ and $Z^{\prime \prime}(\omega)$ should agree with their respective experimental results in both the Nyquist and Bode planes; (3) the parameters, such as the polarizability, $\alpha$, the electric field strength across barrier layer, $\varepsilon$, the standard rate constants, $k_{i}^{00}$, the transfer coefficients, $\alpha_{i}$, and the constant $\phi_{B O I}^{0}$ should be approximately potentialindependent; (4) the calculated current densities and passive film thickness, as estimated from the parameter values obtained from the optimization, should be in reasonable agreement with the steady-state experimental values.

\subsubsection{Results and Discussion}

Typical experimental impedance spectra for the passive state on zirconium in high temperature, de-aerated hydrogen-added borate buffer aqueous solution $\left(0.1 \mathrm{M} \mathrm{B}(\mathrm{OH})_{3}+\right.$ $0.001 \mathrm{M} \mathrm{LiOH}$ solution with $22 \mathrm{~cm}^{3}(\mathrm{STP}) / \mathrm{kg}\left(\mathrm{H}_{2} \mathrm{O}\right)$ hydrogen at $\left.250{ }^{\circ} \mathrm{C}, \mathrm{pH}=6.94\right)$ are shown in Figure 2-9, in the form of Nyquist plots and Bode plots, respectively. Typical 
experimental impedance spectra for the passive state on zirconium $\left(0.1 \mathrm{M} \mathrm{B}(\mathrm{OH})_{3}+\right.$ $0.001 \mathrm{M} \mathrm{LiOH}$ solution at $250{ }^{\circ} \mathrm{C}, \mathrm{pH}=6.94$ ) are shown in Figure 2-10, in which the current due to cation interstitials and other ions is assumed to be $0.2 \%$ of that due to oxygen vacancies. In Nyquist plots, the imaginary component of the impedance is plotted versus the real component with decreasing frequencies. In Bode plots, the total impedance and the $\theta$ (i.e. $\arctan \left(-Z_{\text {imag }} / Z_{\text {real }}\right)$ are all plotted versus the frequency. Also plotted in these figures are impedance data calculated from the model described above using the optimized model parameters. From these figures, it can be seen that the simulated data agrees well with the experimental data, which is similar to that reported in other studies [12,13]. Thus, the impedance model based on the PDM provides a reasonable account of the experimental data.

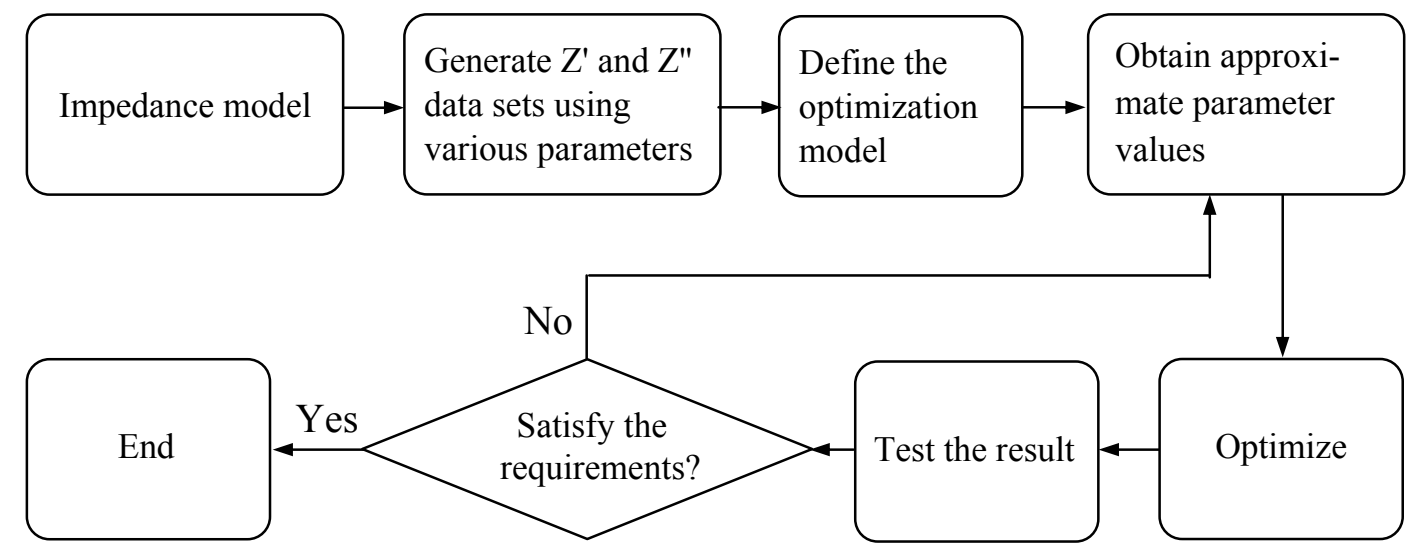

Figure 2-8. Flow chart of optimization of the Point Defect Model on the experimental electrochemical impedance data

The simulated thickness of passive film is very close to its experimental value, as shown in Figures 2-11(a) and 2-12(a). Likewise, the calculated current density, based on the fitting parameter values, also approaches its steady-state experimental result, $I_{s s}$, which is shown in Figures 2-11(b) and 2-12(b). Furthermore, the porosity of outer layer, resistance of solution of the experimental system, polarizability, the electric field strength across barrier layer, standard rate constants, transfer coefficients and $\phi_{B O I}^{0}$ obtained by optimization of the model on the experimental impedance data are all found to be essentially potential-independent within the applied potential range, which, in itself, is a test of the viability of the model described above. Moreover, in the de-aerated aqueous solutions, the thickness of the barrier layer is found to increase with increasing applied potential, but in the hydrogenated aqueous solutions, the thickness of the barrier layer decrease with increasing applied potential, which is consistent with the predictions of the PDM for passive films on metals and alloys [14]. The parameter values have been averaged to yield a single set for passive zirconium, which is shown in Tables 2-3 and 24. It should be noted that the value for $\beta$ was assumed to be -0.005 [12] and the assumption is due to the fact that we do not have any data upon which the model can be optimized with respect to $\mathrm{pH}$ as an independent variable. 

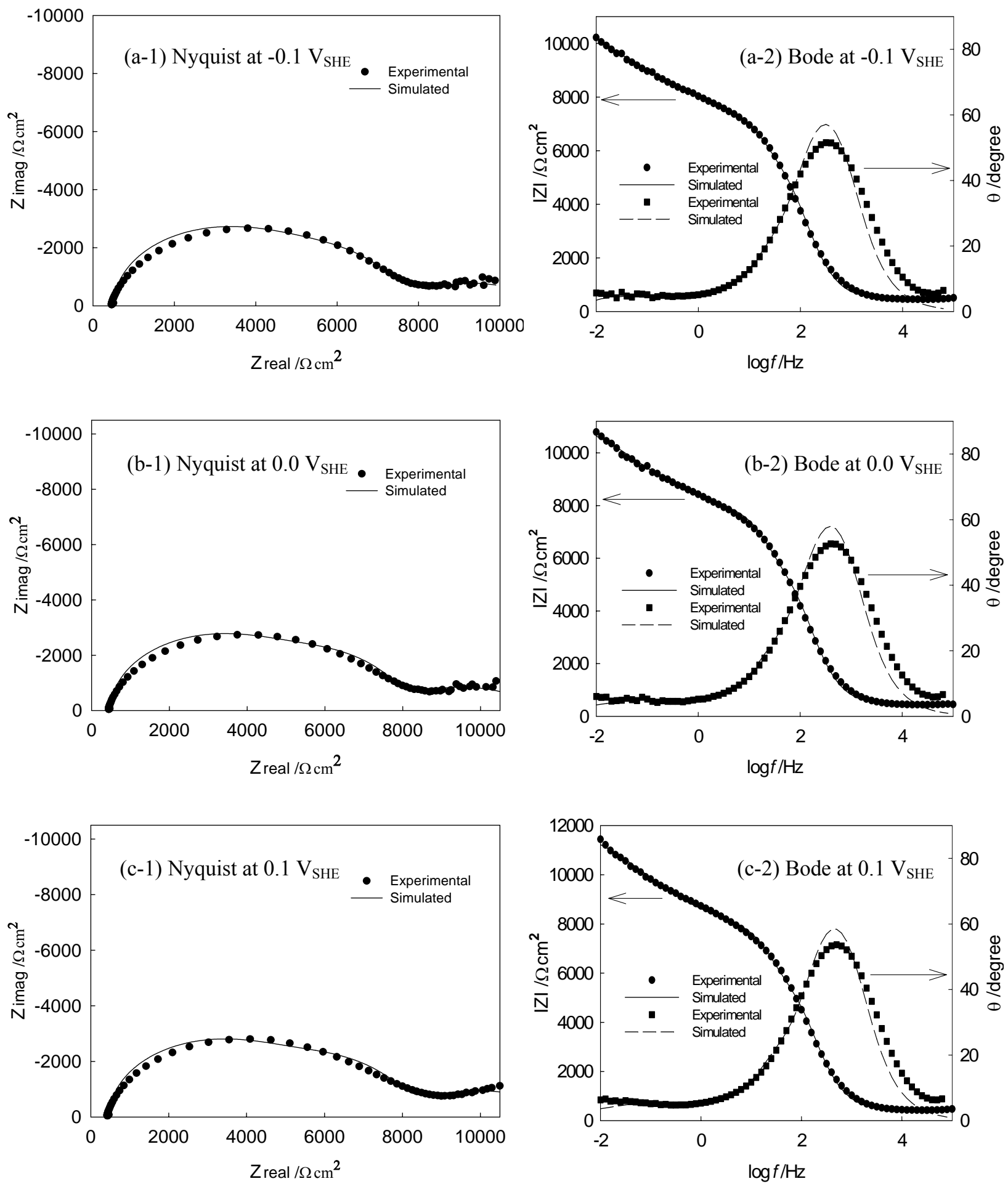

Figure 2-9. Nyquist and Bode impedance plots for zirconium passive film polarized at each potential for 24 hours for film formation in $0.1 \mathrm{M} \mathrm{B}(\mathrm{OH})_{3}+0.001 \mathrm{M} \mathrm{LiOH}$ solutions with $22 \mathrm{~cm}^{3}$ (STP)/ $\mathrm{kg}\left(\mathrm{H}_{2} \mathrm{O}\right)$ hydrogen at $250{ }^{\circ} \mathrm{C}$ and 62 bar and as a function of potential, showing a comparison between the experimental and simulated data. 

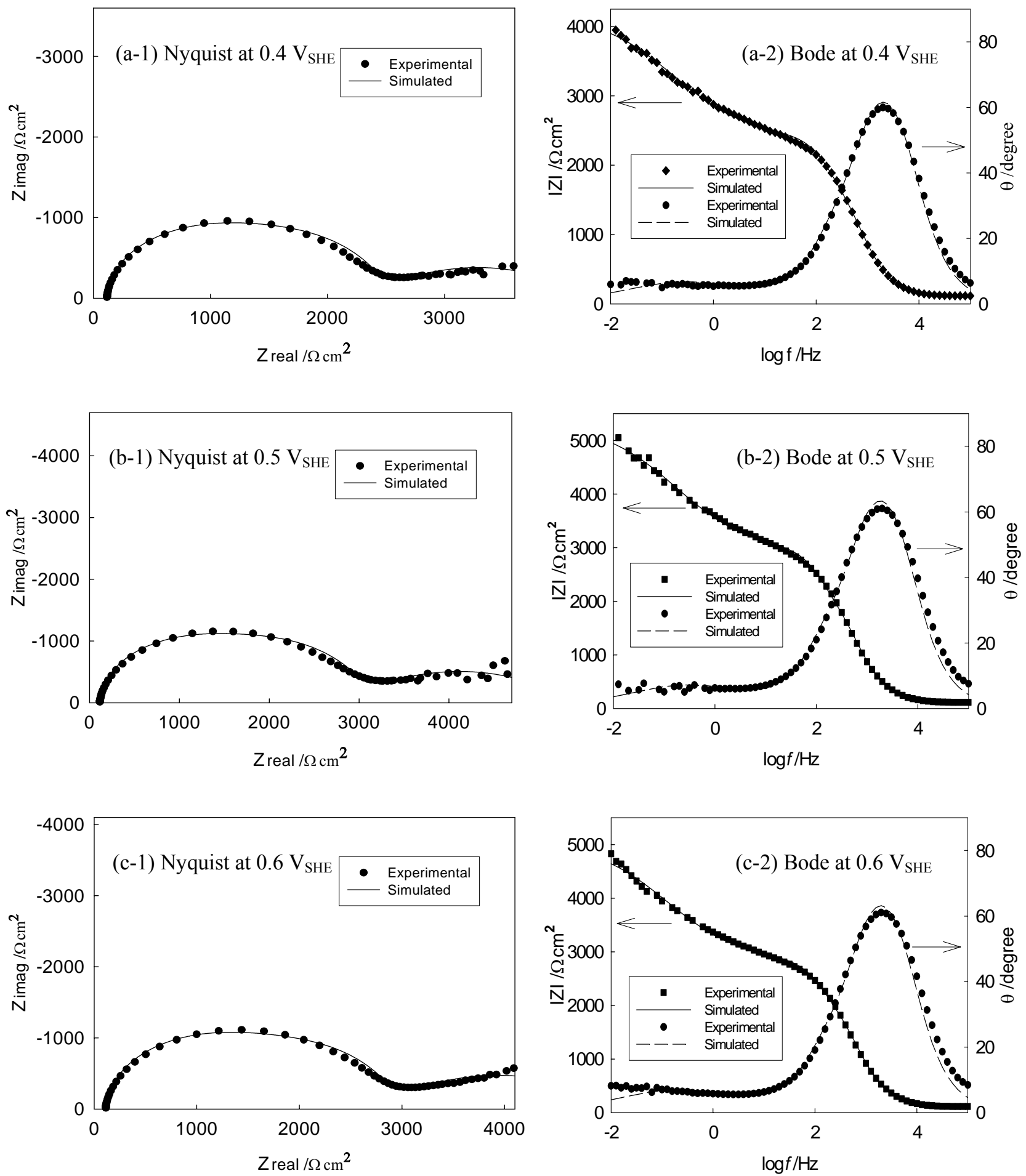

Figure 2-10. Nyquist and Bode impedance plots for zirconium passive film polarized at each potential for 24 hours for film formation in $0.1 \mathrm{M} \mathrm{B}(\mathrm{OH})_{3}+0.001 \mathrm{M} \mathrm{LiOH}$ solutions at $250{ }^{\circ} \mathrm{C}$ and 62 bar and as a function of potential, showing a comparison between the experimental and simulated data. 
Table 2-3 Parameter values after optimization of the point defect model on the experimental impedance data for zirconium in de-aerated hydrogen-added aqueous solution $\left(0.1 \mathrm{M} \mathrm{B}(\mathrm{OH})_{3}\right.$ $+0.001 \mathrm{M} \mathrm{LiOH}$ solution with $22 \mathrm{~cm}^{3}(\mathrm{STP}) / \mathrm{kg}\left(\mathrm{H}_{2} \mathrm{O}\right)$ hydrogen at $\left.250{ }^{\circ} \mathrm{C}, \mathrm{pH}=6.94\right)$

\begin{tabular}{|c|c|c|c|c|}
\hline $\mathrm{E}_{\text {app }}(\mathrm{V})$ vs. SHE & -0.1 & 0 & 0.1 & Mean $( \pm \mathrm{SD})$ \\
\hline Capacitance of barrier layer $\left(\mathrm{F} / \mathrm{cm}^{2}\right)$ & $3.03 \times 10^{-6}$ & $2.55 \times 10^{-6}$ & $2.28 \times 10^{-6}$ & \\
\hline Capacitance of outer layer $\left(\mathrm{F} / \mathrm{cm}^{2}\right)$ & $3.56 \times 10^{-7}$ & $2.87 \times 10^{-7}$ & $2.47 \times 10^{-7}$ & \\
\hline Thickness of barrier layer $(\mathrm{cm})$ & $1.29 \times 10^{-6}$ & $1.24 \times 10^{-6}$ & $1.15 \times 10^{-6}$ & \\
\hline Thickness of outer layer $(\mathrm{cm})$ & $1.44 \times 10^{-5}$ & $1.47 \times 10^{-5}$ & $1.50 \times 10^{-5}$ & \\
\hline Porosity of outer layer, $P$ & 0.408 & 0.401 & 0.405 & \\
\hline Resistance of solution, $R_{b s},(\Omega)$ & 510 & 489 & 475 & $491 \pm 19$ \\
\hline Resistance of outer layer $(\Omega)$ & 4346 & 4476 & 4553 & $(4.46 \pm 0.11) \times 10^{3}$ \\
\hline Warburg coefficient, $\sigma$ & 4736 & 5094 & 5621 & $(5.15 \pm 0.47) \times 10^{3}$ \\
\hline Impedance due to electrons and holes $(\Omega)$ & 6140 & 6643 & 7250 & $(6.68 \pm 0.57) \times 10^{3}$ \\
\hline Polarizability, $\alpha$ & 0.326 & 0.383 & 0.406 & $0.372 \pm 0.046$ \\
\hline$\beta(\mathrm{V})$ & -0.005 & -0.005 & -0.005 & -0.005, Assumed \\
\hline Electric field across barrier layer, $\varepsilon,(\mathrm{V} / \mathrm{cm})$ & -767840 & -721862 & -727921 & $-(0.739 \pm 0.029) \times 10^{6}$ \\
\hline Kinetic order, $m$ & 0.453 & 0.483 & 0.488 & $0.475 \pm 0.022$ \\
\hline$\phi_{B O I}^{0}(\mathrm{~V})$ & -0.029 & -0.025 & -0.036 & $-0.030 \pm 0.004$ \\
\hline Standard rate constant, $k_{1}^{00}\left(\mathrm{~mol} \mathrm{~s}^{-1} \mathrm{~cm}^{-2}\right)$ & $3.59 \times 10^{-15}$ & $3.39 \times 10^{-15}$ & $2.20 \times 10^{-15}$ & $(3.06 \pm 0.86) \times 10^{-15}$ \\
\hline Standard rate constant, $k_{2}^{00}\left(\mathrm{~mol} \mathrm{~s}^{-1} \mathrm{~cm}^{-2}\right)$ & $2.20 \times 10^{-14}$ & $1.85 \times 10^{-14}$ & $1.68 \times 10^{-14}$ & $(1.91 \pm 0.29) \times 10^{-14}$ \\
\hline Standard rate constant, $k_{5}^{00}\left(\mathrm{~mol} \mathrm{~s}^{-1} \mathrm{~cm}^{-2}\right)$ & $2.84 \times 10^{-4}$ & $1.85 \times 10^{-4}$ & $1.82 \times 10^{-4}$ & $(2.17 \pm 0.67) \times 10^{-4}$ \\
\hline Transfer coefficient of Reaction (1), $\alpha_{1}$ & 0.339 & 0.317 & 0.280 & $0.312 \pm 0.032$ \\
\hline Transfer coefficient of Reaction (2), $\alpha_{2}$ & 0.326 & 0.318 & 0.317 & $0.320 \pm 0.006$ \\
\hline Transfer coefficient of Reaction (5), $\alpha_{5}$ & 0.185 & 0.210 & 0.237 & $0.211 \pm 0.026$ \\
\hline
\end{tabular}

According to Equation (2-95) [15], the diffusivity of hydrogen anion vacancies in the passive film, $D$, can be calculated to be in the range from $8.77 \times 10^{-15} \mathrm{~cm}^{2} / \mathrm{s}$ to $1.18 \times$ $10^{-14} \mathrm{~cm}^{2} / \mathrm{s}$ within the applied potential range from -0.1 to $0.1 \mathrm{~V}_{\mathrm{SHE}}$ in the above high temperature, hydrogen-added borate buffer solution, and the diffusivity of oxygen anion vacancies in the passive film can be calculated to be in the range from $8.61 \times 10^{-14} \mathrm{~cm}^{2} / \mathrm{s}$ to $1.02 \times 10^{-13} \mathrm{~cm}^{2} / \mathrm{s}$ within the applied potential range from 0.4 to $0.6 \mathrm{~V}_{\mathrm{SHE}}$ in the above high temperature, de-aerated borate buffer solution. Figure 2-13 shows a comparison of the oxygen anion vacancy diffusivities between our data and those of other workers [1619].

$$
D=2 \sigma^{2}(1-\alpha)^{2} I_{s s}^{2} / \varepsilon^{2}
$$

According to Equation (2-96), polarization resistance, $R_{p}$, can be calculated to be 10666, 11118 and $11803 \Omega$, respectively, in the high temperature, hydrogen-added borate buffer solution when the potential is $-0.1,0.0$ and $0.1 \mathrm{~V}_{\mathrm{SHE}}$, and 4071,5211 and $4940 \Omega$, respectively, in the high temperature, de-aerated borate buffer solution when the potential is $0.4,0.5$ and $0.6 \mathrm{~V}_{\text {SHE }}$. The calculation indicates that the polarization resistance varies 
Table 2-4 Parameter values after optimization of the point defect model on the experimental impedance data for zirconium in de-aerated aqueous solution $(0.1 \mathrm{M}$ $\mathrm{B}(\mathrm{OH})_{3}+0.001 \mathrm{M} \mathrm{LiOH}$ solution at $\left.250{ }^{\circ} \mathrm{C}, \mathrm{pH}=6.94\right)$

\begin{tabular}{|c|c|c|c|c|}
\hline $\mathrm{E}_{\text {app }}(\mathrm{V})$ vs. SHE & 0.4 & 0.5 & 0.6 & Mean $( \pm \mathrm{SD})$ \\
\hline Capacitance of barrier layer $\left(\mathrm{F} / \mathrm{cm}^{2}\right)$ & $1.01 \times 10^{-6}$ & $1.00 \times 10^{-6}$ & $1.08 \times 10^{-6}$ & \\
\hline Capacitance of outer layer $\left(\mathrm{F} / \mathrm{cm}^{2}\right)$ & $2.08 \times 10^{-7}$ & $2.09 \times 10^{-7}$ & $1.90 \times 10^{-7}$ & \\
\hline Thickness of barrier layer $(\mathrm{cm})$ & $1.11 \times 10^{-7}$ & $1.52 \times 10^{-7}$ & $1.61 \times 10^{-7}$ & \\
\hline Thickness of outer layer $(\mathrm{cm})$ & $1.63 \times 10^{-5}$ & $1.79 \times 10^{-5}$ & $1.82 \times 10^{-5}$ & \\
\hline Porosity of outer layer, $P$ & 0.396 & 0.373 & 0.387 & \\
\hline Resistance of solution, $R_{b s},(\Omega)$ & 121.4 & 125.2 & 124.5 & $123.7 \pm 2.3$ \\
\hline Resistance of outer layer $(\Omega)$ & 1290 & 1510 & 1550 & \\
\hline Warburg coefficient, $\sigma$ & 2990 & 3927 & 3040 & $(3.32 \pm 0.60) \times 10^{3}$ \\
\hline Impedance due to electrons and holes $(\Omega)$ & 2784 & 3697 & 3384 & \\
\hline Polarizability of the BOI, $\alpha$ & 0.188 & 0.195 & 0.194 & $0.192 \pm 0.004$ \\
\hline$\beta(\mathrm{V})$ & -0.005 & -0.005 & -0.005 & -0.005, Assumed \\
\hline Electric field across barrier layer, $\varepsilon,(\mathrm{V} / \mathrm{cm})$ & 492871 & 564288 & 519162 & $(5.25 \pm 0.39) \times 10^{5}$ \\
\hline$\phi_{B O I}^{0}(\mathrm{~V})$ & -0.243 & -0.181 & -0.175 & $-0.200 \pm 0.043$ \\
\hline Standard rate constant, $k_{2}^{00}\left(\mathrm{~mol} \mathrm{~s}^{-1} \mathrm{~cm}^{-2}\right)$ & $2.50 \times 10^{-15}$ & $2.25 \times 10^{-15}$ & $2.52 \times 10^{-15}$ & $(2.42 \pm 0.17) \times 10^{-15}$ \\
\hline Transfer coefficient of Reaction (2), $\alpha_{2}$ & 0.277 & 0.271 & 0.262 & $0.270 \pm 0.008$ \\
\hline
\end{tabular}
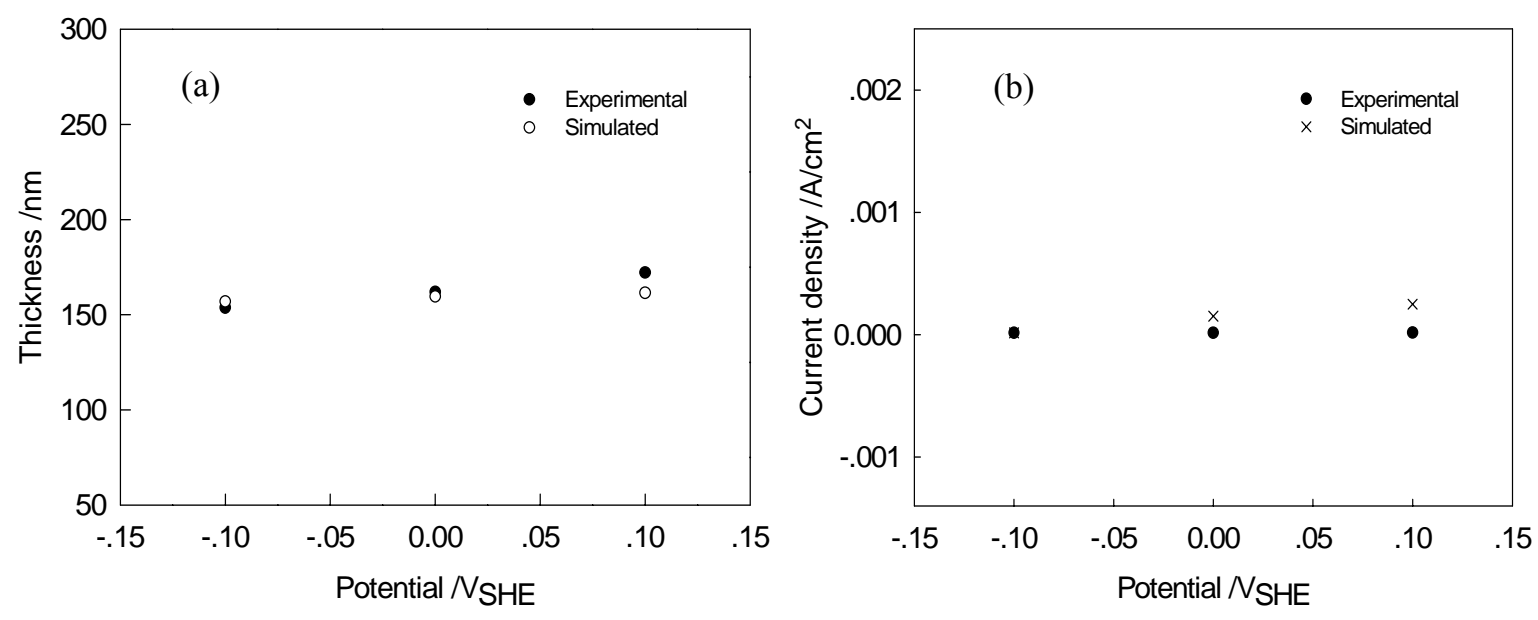

Figure 2-11. Comparison between the experimental and simulated data, (a) the thickness of passive film; (b) the current density, where the solution is $0.1 \mathrm{M} \mathrm{B}(\mathrm{OH})_{3}+0.001 \mathrm{M}$ $\mathrm{LiOH}$ aqueous solution with $22 \mathrm{~cm}^{3}(\mathrm{STP}) / \mathrm{kg}\left(\mathrm{H}_{2} \mathrm{O}\right)$ hydrogen at $250^{\circ} \mathrm{C}, \mathrm{pH}=6.94$.

little with the polarizability, transfer coefficients, and the electric field strength across the barrier layer, but it does change significantly with the porosity and thickness of the outer layer. For examples, in the high temperature, hydrogen-added borate buffer solution 
when the potential is $0.0 \mathrm{~V}_{\mathrm{SHE}}$, the polarization resistance decreases from $42547 \Omega$ to $24419 \Omega$ to $11118 \Omega$, and eventually to $10226 \Omega$ when the porosity increases from 0.050 to 0.101 to 0.401 , and eventually to 0.501 , while all other model parameter values are the same as those shown in Table 2-3. In the high temperature, deaerated borate buffer solution when the potential is $0.4 \mathrm{~V}_{\mathrm{SHE}}$, the polarization resistance decreases from $8105 \Omega$ to $5386 \Omega$ to $4071 \Omega$, and eventually to $3516 \Omega$, when the porosity increases from 0.096 to 0.1960 o 0.396 , and eventually to 0.696 , while all other model parameter values are the same as those shown in Table 2-4. These data indicate that the corrosion resistance of zirconium in the two aqueous solutions is dominated by the outer layer.

$$
R_{p}=Z(\omega=0)-Z(\omega=\infty) \approx Z\left(\omega=10^{-20}\right)-Z\left(\omega=10^{20}\right)
$$
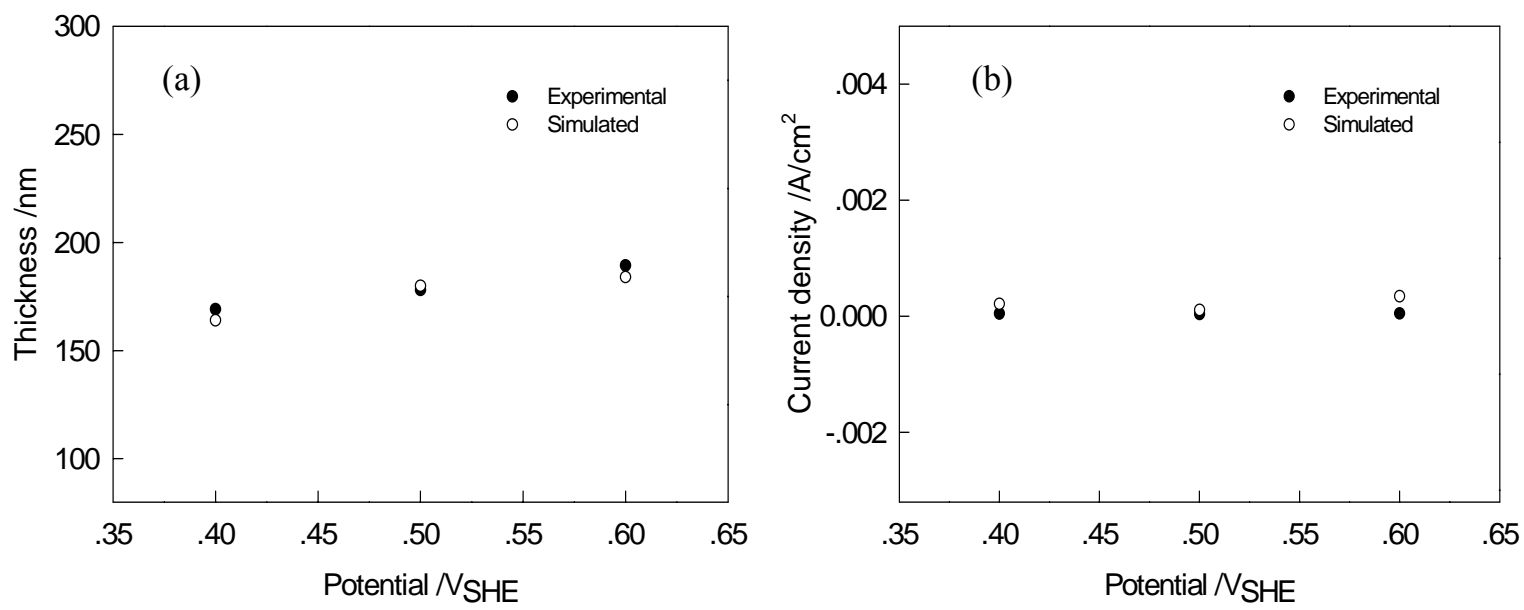

Figure 2-12. Comparison between the experimental and simulated data, (a) the thickness of passive film; (b) the current density, where the solution is $0.1 \mathrm{M} \mathrm{B}(\mathrm{OH})_{3}+$ $0.001 \mathrm{M} \mathrm{LiOH}$ aqueous solution at $250^{\circ} \mathrm{C}, \mathrm{pH}=6.94$.

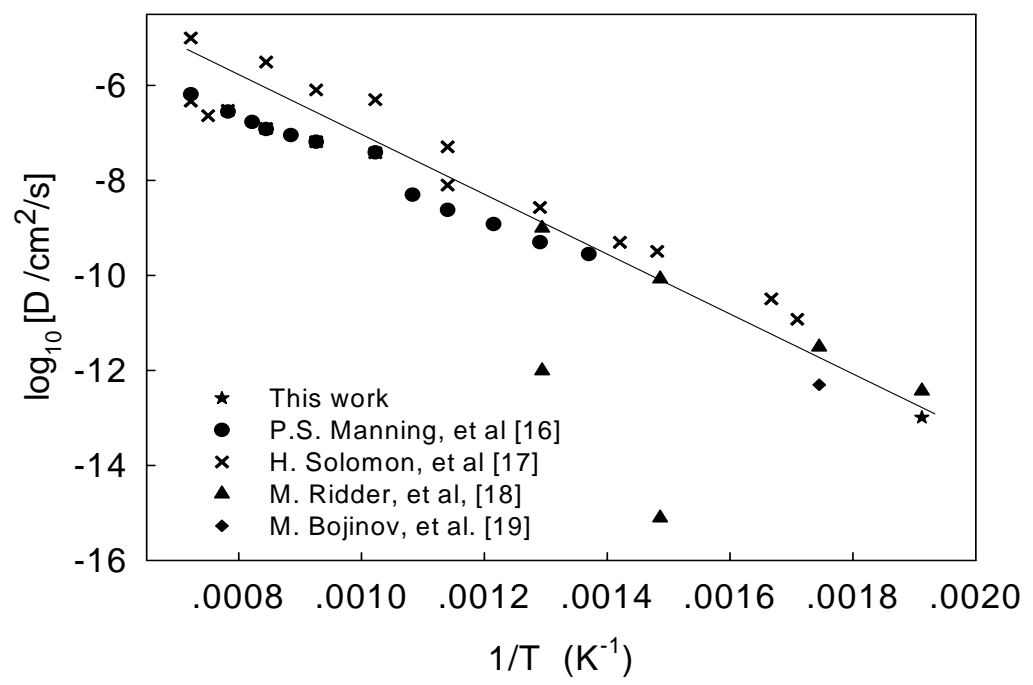

Figure 2-13 Comparison of oxygen vacancy diffusivity obtained in this study and those from literatures [16-19]. 

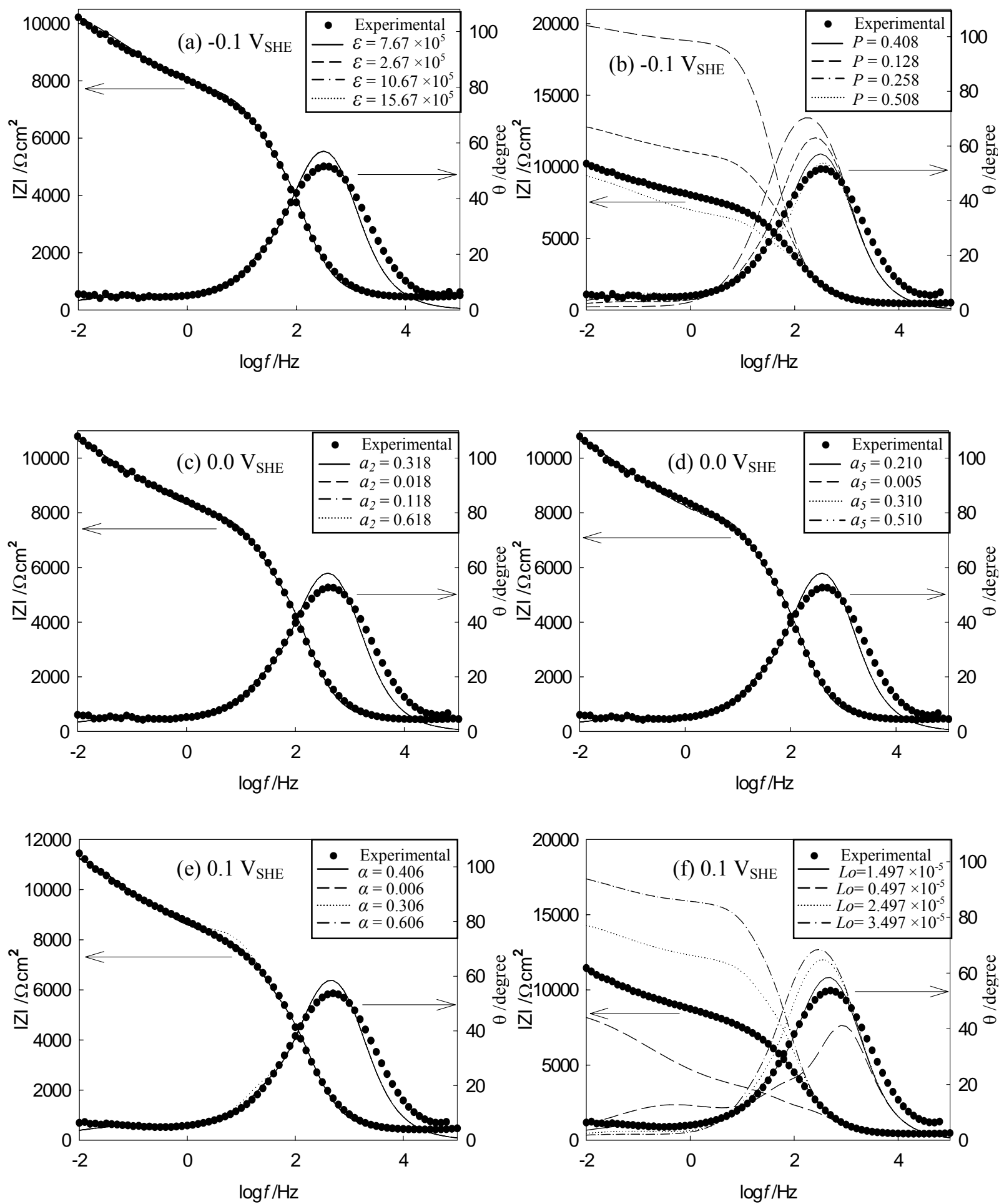

Figure 2-14. Sensitivity analysis, showing the effect of model parameter value change on the fitting curve, where the solution is $0.1 \mathrm{M} \mathrm{B}(\mathrm{OH})_{3}+0.001 \mathrm{M} \mathrm{LiOH}$ aqueous solution with $22 \mathrm{~cm}^{3}(\mathrm{STP}) / \mathrm{kg}\left(\mathrm{H}_{2} \mathrm{O}\right)$ hydrogen at $250^{\circ} \mathrm{C}, \mathrm{pH}=6.94$. 

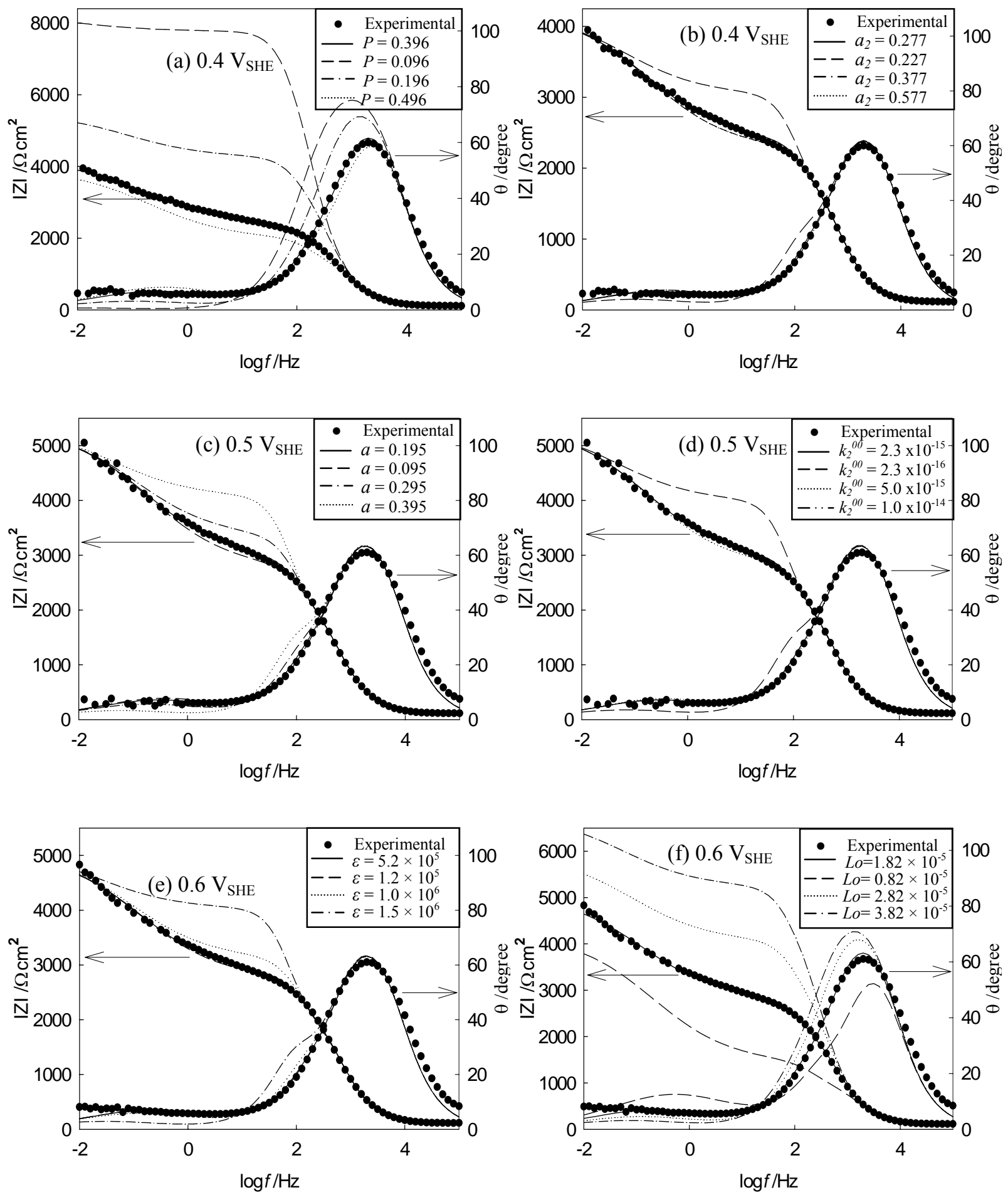

Figure 2-15. Sensitivity analysis, showing the effect of model parameter value change on the fitting curve, where the solution is $0.1 \mathrm{M} \mathrm{B}(\mathrm{OH})_{3}+0.001 \mathrm{M} \mathrm{LiOH}$ aqueous solution at $250{ }^{\circ} \mathrm{C}, \mathrm{pH}=6.94$. 
In order to rank the model parameters that have the greatest impact on the predictions of the model, extensive sensitivity analysis was carried out. Typical effects of changing the model parameter value on the calculated spectra are shown in Figures 214 and 2-15, in which the other model parameter values are the same as those shown in Tables 2-3 and 2-4, respectively. Also plotted in these figures are experimental impedance data. It can be seen from these figures that the change of the porosity and the thickness of the outer layer with reference to the results listed in Tables 2-3 and 2-4 leads to the greatest deviation of the fitting curves from the experimental results, which indicates again that the corrosion resistance is dominated by the outer layer.

The principal findings of this part are as follows:

- The impedance model based on the PDM provides a reasonable account of the growth of bilayer passive films formed on zirconium and Zircaloys under PWR and BWR coolant conditions.

- The corrosion resistance of zirconium in high temperature, de-aerated and hydrogenated aqueous solutions is dominated by the porosity and thickness of the outer layer.

- The porosity of the outer layer, the electric field strength across the barrier layer, the polarizability of the barrier layer/outer layer interface, the standard rate constants, transfer coefficients for the point defect generation and annihilation at the barrier layer interfaces, and other parameters are found to be essentially constant within the applied potential range explored in this work.

- Optimization of the impedance model on the experimental data has yielded a set of parameter values that might be used for predicting the accumulation of general corrosion damage to Zircaloy fuel sheath under PWR and BWR operating conditions.

\subsection{Stability Analysis of the Passive Films}

It is well known that zirconium is characterized by highly negative Gibbs energies of reaction with oxygen and water under common conditions [20,21], demonstrating that the formation of the oxides from zirconium is thermodynamically spontaneous. It is passivity that enables zirconium and its alloys to be used in oxidizing environments, and it is generally accepted that passivity is due to the formation of a thin oxide film on the surface. In spite of quite a number of work on the passivity of zirconium, no model had emerged to provide a scientific basis for reasonably predicting the behavior of passive zirconium in real aqueous environments. Although Pourbaix diagrams have proven to be powerful tools in analyzing corrosion electrochemical phenomena, they take the equilibrium of electrochemical systems as the precondition, where the formation of passive films is governed by equilibrium thermodynamics, thus they can only provide equilibrium thermodynamic descriptions for the tendency of being corroded. The passivity is a kinetic phenomenon [20,21], and the formation of passive films is a dynamically stable or meta-stable process. Therefore, some limitations inevitably exist 
while applying Pourbaix diagrams to interpret the passivity. In this part, Point Defect Model (PDM) was applied to explore the conditions for the use of zirconium and its alloys in high temperature aqueous solutions using phase-space analysis (PSA).

In order to simplify the analysis, only five primary interfacial reactions shown in Fig.2-5 or Fig.2-7 are taken into consideration.

\subsubsection{Passive Films Formed Under BWR Coolant Conditions}

The thickness change rate, $d L / d t$, of the barrier layer that forms irreversibly on zirconium surface is equal to its growth rate, $d L^{+} / d t$, at the MBI, minus its dissolution rate, $d L^{-} / d t$, at the BOI, as shown in Fig.2-7:

$$
d L / d t=d L^{+} / d t-d L^{-} / d t=\Omega k_{2}^{0} e^{a_{2}\left(V-I R_{o l}\right)} e^{b_{2} L} e^{c_{2} p H}-\Omega k_{5}^{0}\left(C_{H^{+}} / C_{H^{+}}^{0}\right)^{n}
$$

where $a_{2}=4 \alpha_{2}(1-\alpha) \cdot \gamma, b_{2}=-4 \alpha_{2} \varepsilon \cdot \gamma$, and $c_{2}=-4 \alpha_{2} \beta \cdot \gamma$, which are derived by the method of partial charge transfer. In this expression, $\Omega=21.06 \mathrm{cc} / \mathrm{mol}$ is the mole volume of the barrier layer per cation; $\varepsilon(\mathrm{V} / \mathrm{cm})$ is the electric field strength across the barrier layer, which is postulated to be a constant and independent of the applied voltage in the steady state, because of the buffering action of band-to-band (Esaki) or band-tointer-bandgap state tunneling [3]; $k_{i}^{0}$ and $\alpha_{i}$ are the standard rate constant and transfer coefficient, respectively, for the appropriate reactions depicted in Figure 2-7; $\alpha$ is the polarizability of the BOI, i.e., the dependence of the voltage drop across the interface, $\phi_{f / s}$, on the applied voltage, $V ; I \cdot R_{o l}$ is the voltage drop across the outer layer; $\beta$ is the dependence of $\phi_{f / s}$ on $p H$ (assumed to be linear); $\gamma=F / R T$ ( $F$ is Faraday's constant); $C_{H^{+}}$ is the concentration of hydrogen ion at the BOI; $C_{H^{+}}^{0}$ is the standard state concentration, and $n$ is the kinetic order of the barrier layer dissolution reaction with respect to $\mathrm{H}^{+}$. Note that the rate of dissolution Reaction (5) (shown in Figure 2-7) is voltage-independent.

By setting the left side of Equation (2-97) equal to zero, the steady state thickness of the barrier layer, $L_{s s}$, is readily derived as

$$
L_{s s}=\left[\frac{1-\alpha}{\varepsilon}\right] \cdot\left(V-I_{s s} R_{o l}\right)+\left[\frac{2.303 n}{4 \alpha_{2} \varepsilon \cdot \gamma}-\frac{\beta}{\varepsilon}\right] p H+\frac{1}{4 \alpha_{2} \varepsilon \cdot \gamma} \ln \left(\frac{k_{2}^{0}}{k_{5}^{0}}\right)
$$

Note that in deriving these expressions, the units conform to our previous convention that, for the rate of barrier layer dissolution, $C_{H^{+}}$and $C_{H^{+}}^{0}$ have units of $\mathrm{mol} / \mathrm{cm}^{3}$, but when used for defining $p H$, the units are the conventional $\mathrm{mol} / \mathrm{l}$.

The steady state passive current density is readily derived as

$$
I_{s s}=4 F\left[k_{1}^{0} e^{a_{1}\left(V-I_{s s} R_{o l}\right)} e^{b_{1} L_{s s}} e^{c_{1} p H}+k_{2}^{0} e^{a_{2}\left(V-I_{s s} R_{o l}\right)} e^{b_{2} L_{s s}} e^{c_{2} p H}\right\rfloor
$$


where the first and second terms arise from the generations of cation interstitials, and oxygen vacancies, respectively.

In applying phase space analysis, the parameter values summarized in Table 2-4 for passive zirconium in high temperature de-aerated aqueous solutions were employed.

Analysis of the stability of the barrier layer can be achieved by plotting Equation (2-97) in "phase space", i.e., by plotting $d L^{+} / d t$ and $d L^{-} / d t$ versus the thickness of the barrier layer, $L$, as a function of the applied voltage, $p H$, and other variables, as may be deemed appropriate. Figure 2-16 displays a schematic phase-space plot of Equation (1), where the dissolution rate is assumed to be potential-independent. From this figure, it can be seen that the first term on the right side of Equation (2-97) exponentially decreases with increasing $L$, whereas the second term remains constant. The point of intersection between $d L^{+} / d t$ and $d L^{-} / d t$ defines the steady-state barrier layer thickness. Because a physically-meaningful value of $L_{s s}$ results from a balance between the rates of barrier layer growth and dissolution at the MBI and BOI, respectively, the film is only kinetically stable/meta-stable. If a transient thickness of the barrier layer, $L$, is less than the steady state thickness, $L_{s s}, d L^{+} / d t>d L^{-} / d t$, and the barrier layer thickness increases gradually, and reaches its steady state thickness eventually. Contrarily, if a transient thickness of the barrier layer, $L$, is larger than the steady state thickness, $L_{s s}$, $d L^{+} / d t<d L^{-} / d t$, and the barrier layer thickness decreases until it reaches its steady state thickness. At the steady state, $d L^{+} / d t=d L^{-} / d t$, and the barrier layer thickness is constant (corresponding to each applied potential). The barrier layer is in a kinetically

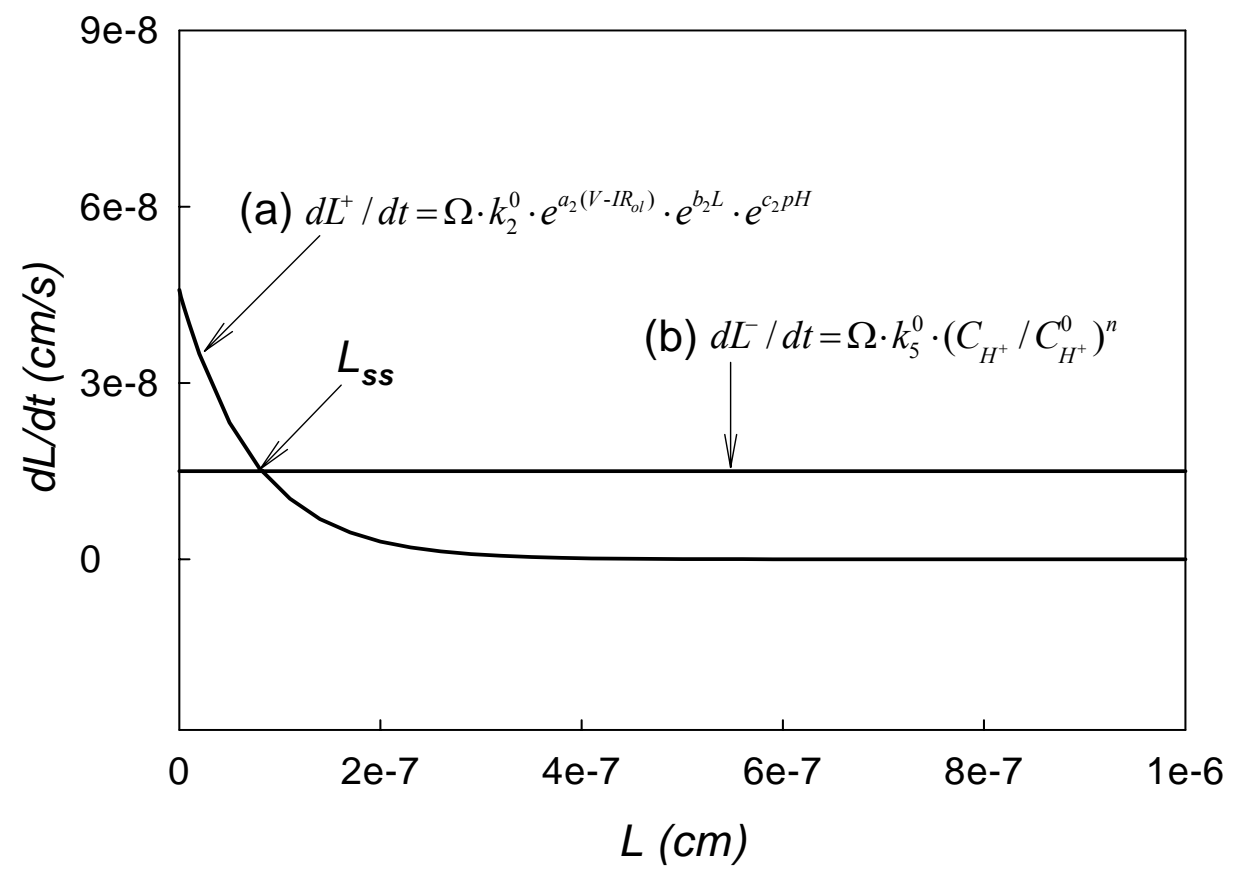

Figure 2-16. Schematic diagram of phase space analysis. The intersection of (a) and (b) defines the steady state thickness of the meta-stable barrier layer. 
stable/meta-stable state, but never in thermo-dynamical equilibrium state. It can be explained by the fact that, for an equilibrium system, the thermodynamic affinities of all processes in the system must be zero simultaneously; that is, the affinities for Reactions (2) and (5), which lead to finite values for $d L^{+} / d t$ and $d L^{-} / d t$, are simultaneously zero. Evidently, $d L^{+} / d t=0$ can only be attained if $L_{s s} \rightarrow \infty$, but $d L^{-} / d t=0$ can never be satisfied for any real substance because all real substances have finite dissolution rates under unsaturated conditions. Accordingly, a passive state at equilibrium is physically non-realizable, which was elaborated by Macdonald [3].

It is evident from Figure 2-16 that the physical condition that must be met for the barrier layer to exist on the surface is

$$
\left(d L^{+} / d t\right)_{L=0}>\left(d L^{-} / d t\right)_{L=L_{s s}}>\left(d L^{+} / d t\right)_{L>L_{s s}}
$$

Equation (2-100) is a statement of the conditions that must exist for the use of reactive metals in aqueous environments and hence the specific conditions that had to have been met for the development of our metals-based civilization. If $\left(\mathrm{dL}^{+} / \mathrm{dt}\right)_{\mathrm{L}=0} \leq\left(d L^{-} / d t\right)_{L=L_{s s}}$, the barrier layer does not exist.

Linear-linear and log-linear phase space plots for zirconium in approximately neutral aqueous solution $\left(0.1 \mathrm{M} \mathrm{B}(\mathrm{OH})_{3}+0.001 \mathrm{M} \mathrm{LiOH}\right)$ de-aerated aqueous solution $\left(\mathrm{pH}=6.94\right.$ at $250{ }^{\circ} \mathrm{C}$ and $\mathrm{P}=62 \mathrm{bar}$ ), as a function of applied potential, are shown in Figures 2-17(a) and (b), respectively. The parameter values used in the calculation are given in Table 2-4. Note that an intersection occurs between $d L^{+} / d t$ and $d L^{-} / d t$ at

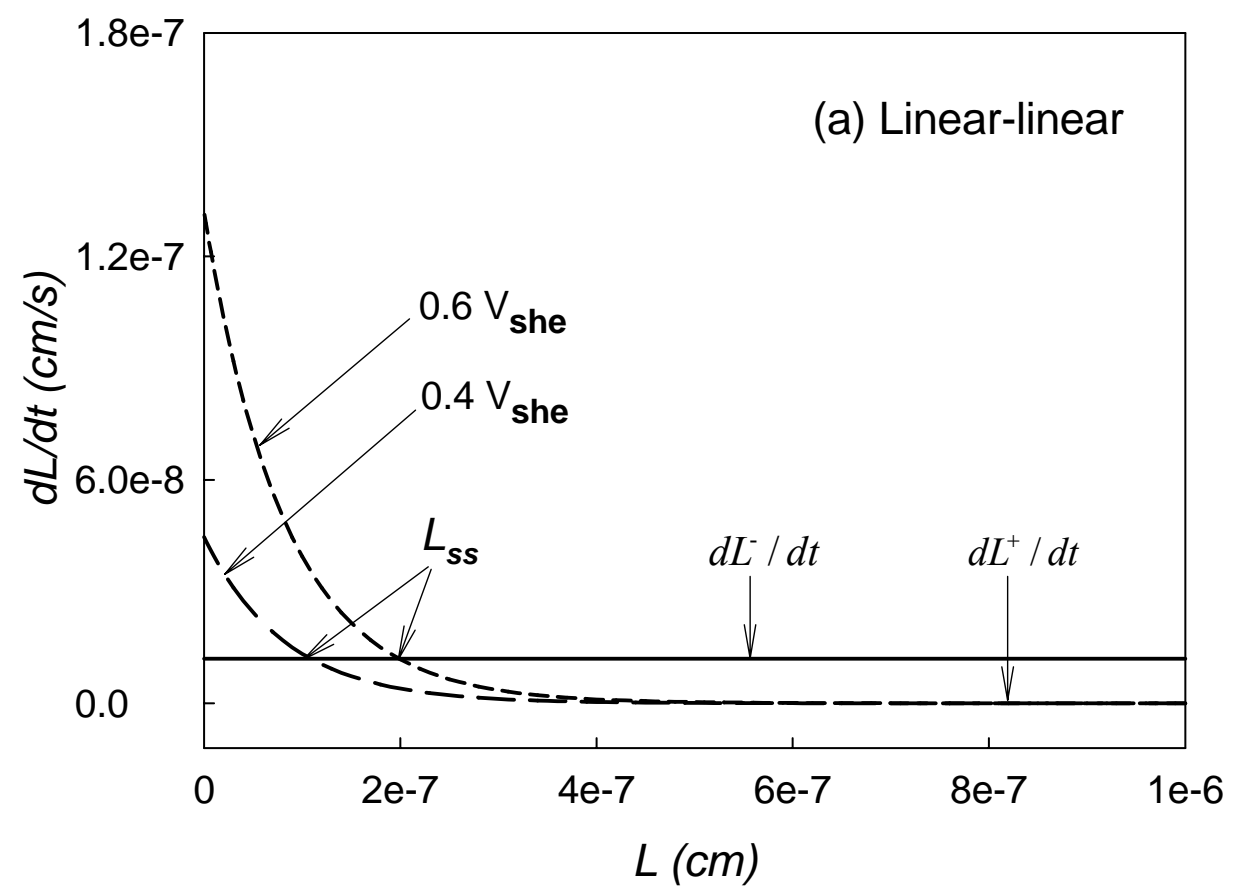




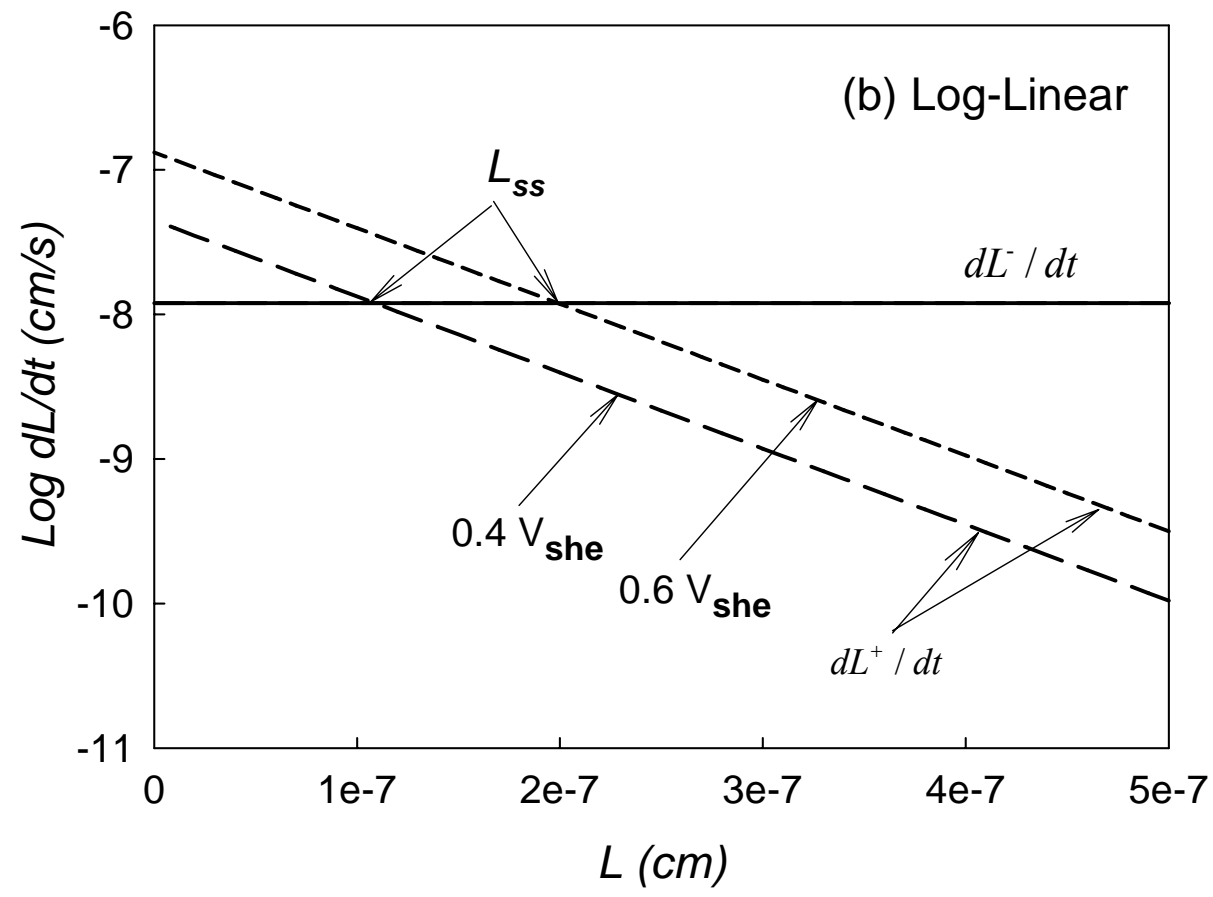

Figure 2-17. Linear-linear (a) and log-linear (b) phase space plots for the barrier layer on zirconium in $0.1 \mathrm{M} \mathrm{B}(\mathrm{OH})_{3}+0.001 \mathrm{M} \mathrm{LiOH}$ de-aerated aqueous solution $(p H=6.94$ at $250^{\circ} \mathrm{C}$ and $\mathrm{P}=62$ bar), as a function of applied potential.

each applied potential, so that a kinetically stable/meta-stable barrier layer exists in all cases. It can be seen from this figure that the dissolution rate of the barrier layer, $d L^{-} / d t$, in the passive state, is potential-independent, whereas the barrier layer growth rate increases with increasing voltage. As noted above, the point of intersection of $d L^{+} / d t$ and $d L^{-} / d t$ defines the steady state thickness of the barrier layer.

\section{Acid Depassivation}

Log-linear phase space plots for zirconium as a function of the $p H$ at the BOI and the barrier layer thickness are shown in Figure 2-18, in which the applied potential is 0.5 $\mathrm{V}_{\mathrm{SHE}}$, and the kinetic order of the barrier layer dissolution reaction with respect to $\mathrm{H}^{+}, n$, is assumed to be 0.6. It can be seen from these plots that at various transient barrier layer thicknesses, the barrier layer growth rate is a weakly increasing function of $p H$, primarily due to the small value of $\beta$, as plotted by the solid lines. But the dissolution rate of the barrier layer is a strongly decreasing function of $p H$, as depicted by the dashed lines. For example, at a potential of $0.5 \mathrm{~V}_{\mathrm{SHE}}$, the dissolution rate of the barrier layer decreases from $10^{-2.87} \mathrm{~cm} / \mathrm{s}$ to $10^{-3.77} \mathrm{~cm} / \mathrm{s}$ to $10^{-4.67} \mathrm{~cm} / \mathrm{s}$ to $10^{-5.87} \mathrm{~cm} / \mathrm{s}$ to $10^{-7.07} \mathrm{~cm} / \mathrm{s}$, and eventually to $10^{-8.27} \mathrm{~cm} / \mathrm{s}$, when the $p H$ at the BOI increases from -2 to -0.5 to 1 to 3 to 5 , and eventually to 7 . The steady state thickness of the barrier layer at $0.5 \mathrm{~V}_{\mathrm{SHE}} \mathrm{vs}$. $p H$ at the 
BOI is plotted in Figure 2-19, where the kinetic order is assumed to be 0.3 . It is evident that the steady state thickness of the barrier layer is predicted to decrease sharply with decreasing $p H$. The barrier layer would be destroyed at $p H$ of depassivation $p H_{\text {depass }}=$ 4.4 if the kinetic order is equal to 0.3. At this point, the barrier layer cannot exist on the surface, even as a kinetically metastable phase, i.e., the surface is depassivated. Thus the substrate dissolves rapidly in the passive-to-active transition, depending upon the applied potential and the kinetics of the metal dissolution reaction. Note that at $0.5 \mathrm{~V}_{\mathrm{SHE}}$, the depassivation is theoretically predicted not to occur at $p H=4.39$, demonstrating the predicted catastrophic nature of depassivation. Note that in the calculation, the kinetic order was assumed not to change with $p H$.

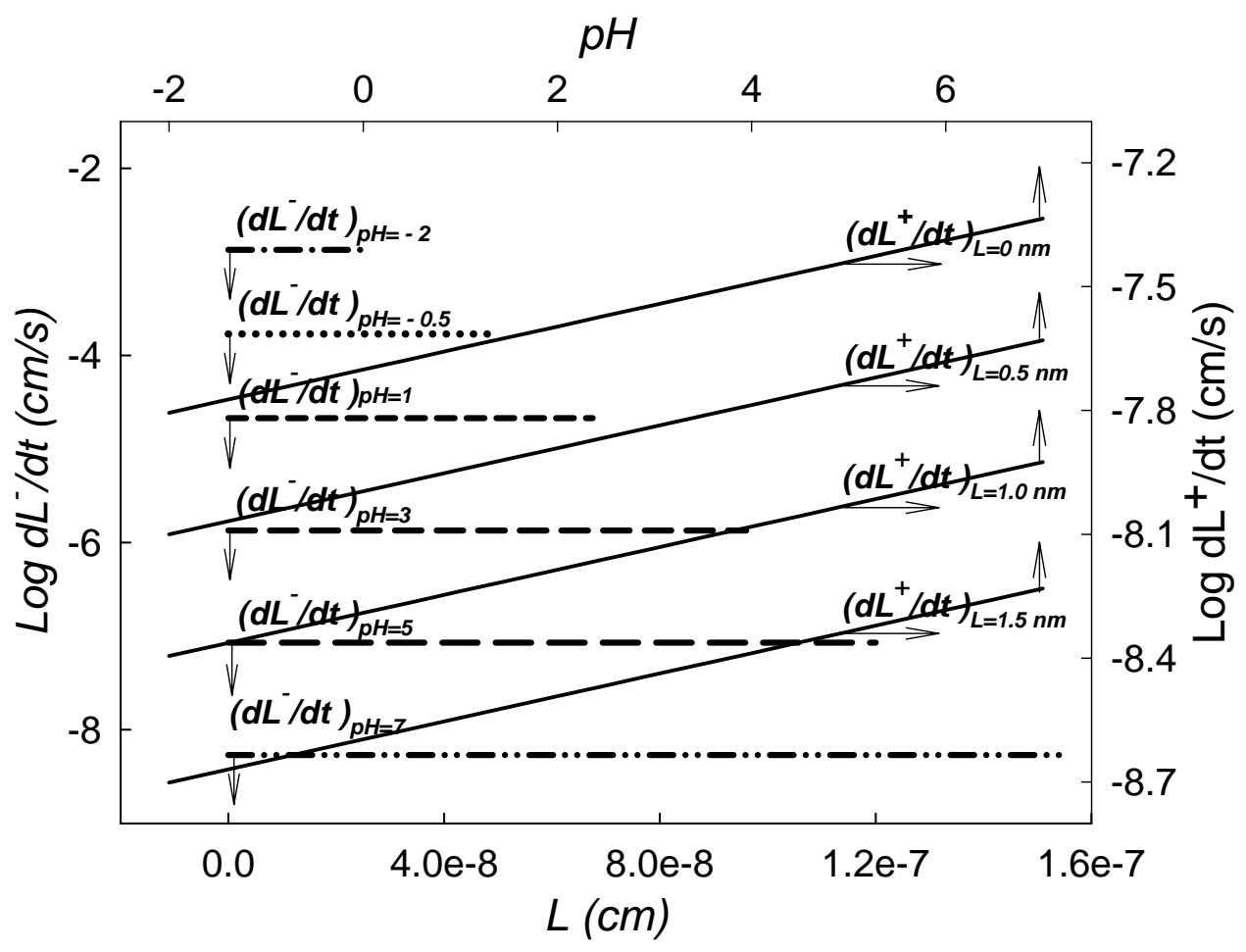

Figure 2-18. Phase space plots for the barrier layer of the passive film on zirconium in $0.1 \mathrm{M} \mathrm{B}(\mathrm{OH})_{3}+0.001 \mathrm{M} \mathrm{LiOH}$ de-aerated aqueous solution $\left(\mathrm{T}=250{ }^{\circ} \mathrm{C}\right.$ and $\mathrm{P}=62$ bar $)$ at $0.5 \mathrm{~V}_{\mathrm{SHE}}$, as a function of the $p H$ at the $\mathrm{BOI}$ and the barrier layer thickness. 


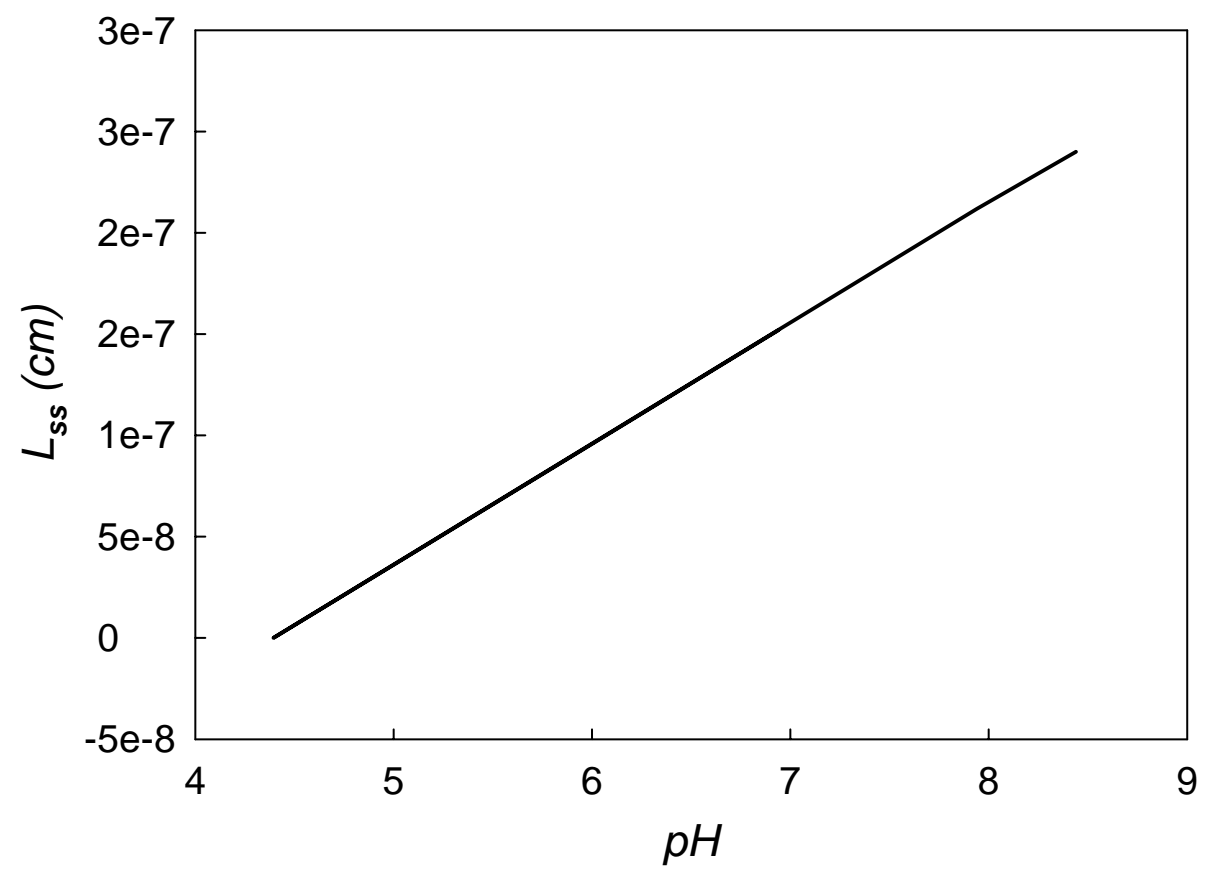

Figure 2-19. Plot of the steady state thickness of the barrier layer on zirconium in $0.1 \mathrm{M}$ $\mathrm{B}(\mathrm{OH})_{3}+0.001 \mathrm{M} \mathrm{LiOH}$ de-aerated aqueous solution $\left(\mathrm{T}=250^{\circ} \mathrm{C}\right.$ and $\mathrm{P}=62$ bar $)$ at 0.5 $\mathrm{V}_{\mathrm{SHE}}$, as a function of $p H$ at the BOI, showing the condition for depassivation $\left(L_{s s} \rightarrow 0\right)$.

Log-linear plot of the total current density as a function of the $p H$ at the BOI are displayed in Figure 2-20. In this instance, the total current density varies exponentially with the $p H$. This is because the current density for the generation of oxygen vacancies at the MBI is not a function of the respective defect concentrations at that interface, whereas it is a function of the defect concentrations at the BOI, and an expression of the current density, based on the reactions at the BOI, would require knowledge of these concentrations [15]. The rate constants for the reactions at the MBI are exponentially dependent upon the voltage, the $p H$, and the barrier layer thickness, with the latter being a linear function of the $p H$ (shown in Figure 2-19). It is the barrier layer thickness that dominates the $p H$-dependence of the current for the generation of oxygen vacancies, and it is for this reason that the current depends exponentially on the $p H$. 


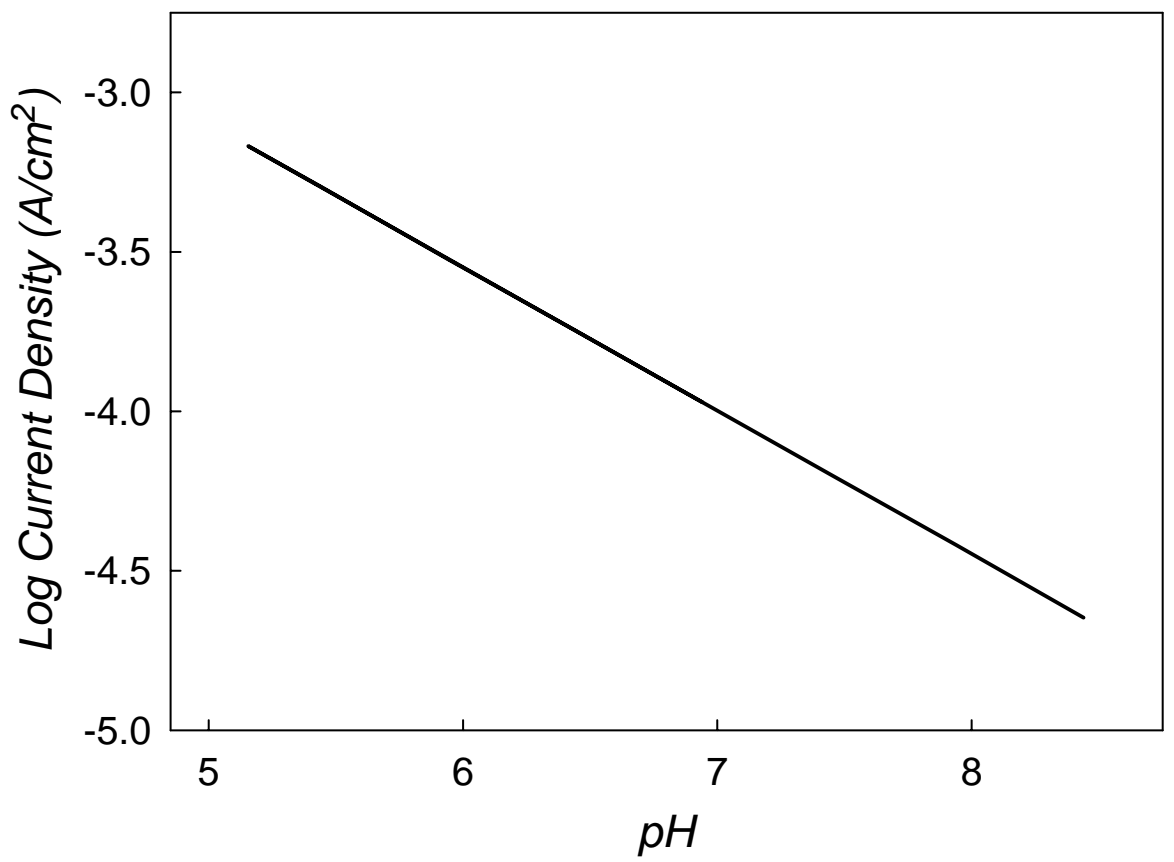

Figure 2-20. Plot of the total current density vs $p H$ for passive zirconium in $0.1 \mathrm{M}$ $\mathrm{B}(\mathrm{OH})_{3}+0.001 \mathrm{M} \mathrm{LiOH}$ de-aerated aqueous solution $\left(\mathrm{T}=250{ }^{\circ} \mathrm{C}\right.$ and $\mathrm{P}=62$ bar $)$ at 0.50 $\mathrm{V}_{\text {SHE. }} n$ is assumed to be 0.45 .

The corrosion rate, $C R$, is readily calculated from the total current density that passes across the interface, as

$$
C R=M \cdot I_{T o t} / 4 F \rho
$$

where $M$, and $\rho$ are the atomic weight, and mass density of zirconium, respectively, and $F$ is Faraday's constant $(F=96,487 \mathrm{C} /$ equiv). Values for $M$, and $\rho$ of $91.22 \mathrm{~g} / \mathrm{mol}$, and $6.511 \mathrm{~g} / \mathrm{cm}^{3}$, respectively, were selected. Taking a voltage of $0.4 \mathrm{~V}_{\text {SHE }}$ as an example, the corrosion rate of zirconium in $0.1 \mathrm{M} \mathrm{B}(\mathrm{OH})_{3}+0.001 \mathrm{M} \mathrm{LiOH}$ de-aerated aqueous solution $\left(\mathrm{T}=250{ }^{\circ} \mathrm{C}\right.$ and $\mathrm{P}=62$ bar $)$ is an exponentially decreasing function of the $p H$. At $0.4 \mathrm{~V}_{\mathrm{SHE}}$, the corrosion rate increases from $8.17 \times 10^{-6} \mu \mathrm{m} / \mathrm{s}$ at $p H=8.44$ to $2.46 \times 10^{-}$ ${ }^{4} \mu \mathrm{m} / \mathrm{s}$ at $p H=5.16$. 


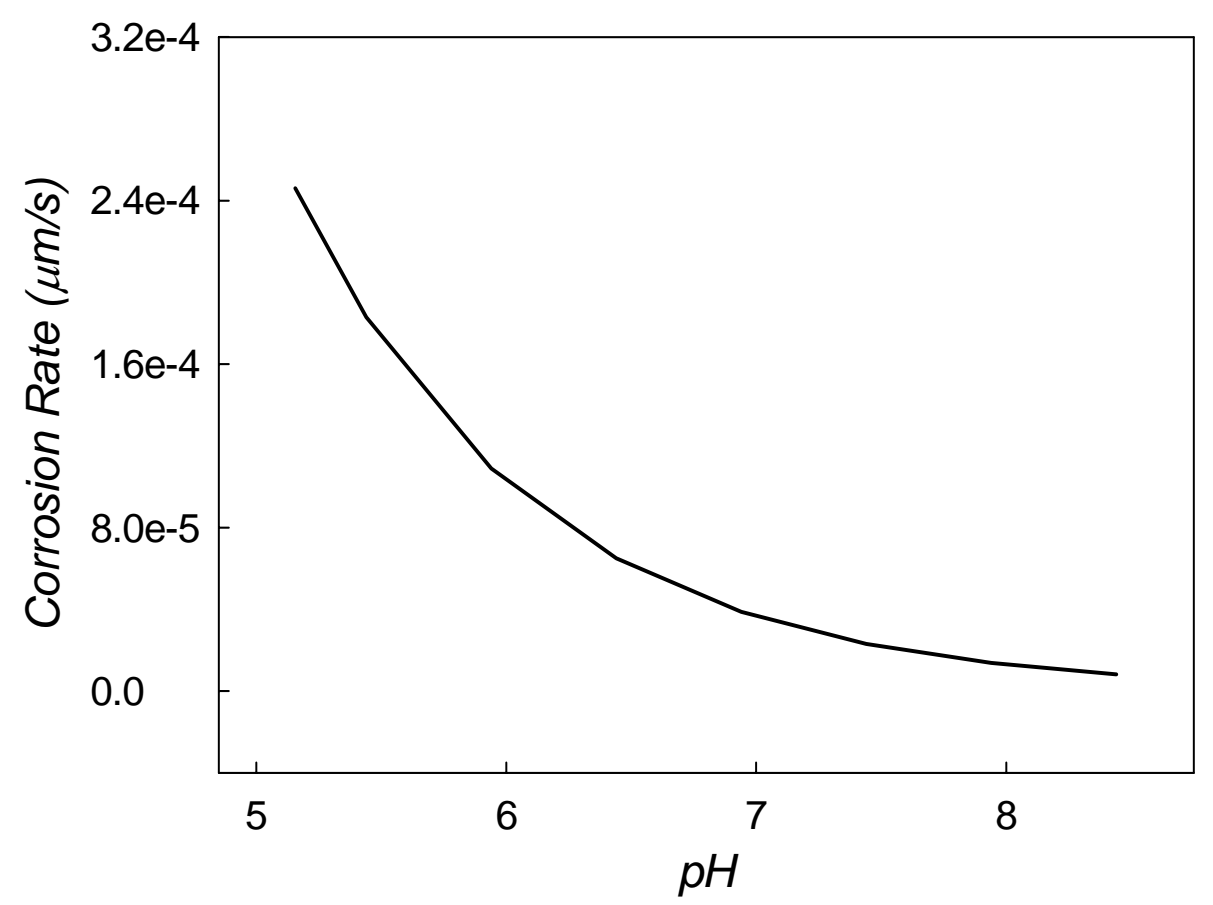

Figure 2-21. Corrosion rate vs $p H$ calculated from the total current density as a function of $p H$ for zirconium in $0.1 \mathrm{M} \mathrm{B}(\mathrm{OH})_{3}+0.001 \mathrm{M} \mathrm{LiOH}$ de-aerated aqueous solution $(\mathrm{T}=$ $250{ }^{\circ} \mathrm{C}$ and $\mathrm{P}=62$ bar) at a voltage of $0.50 \mathrm{~V}_{\mathrm{SHE}}$. Note that the corrosion rate is predicted to be an exponentially decreasing function of $p H$, and $n$ is assumed to be 0.45 .

\section{Kinetic Stability Diagram}

As described above, Pourbaix (potential-pH) diagrams have proven to be powerful tools in analyzing corrosion electrochemical phenomena. However, the equilibrium of electro-chemical systems is taken as the precondition, where the formation of passive films is governed by equilibrium thermodynamics, thus these diagrams can only provide equilibrium thermodynamic descriptions for the tendency of being corroded. The passivity is a kinetic phenomenon [20,21], and the formation of passive films is a dynamically stable or meta-stable process. Therefore, there are inevitably some limitations of Pourbaix diagrams in interpreting passivity [3].

It was demonstrated above that the conditions for depassivation given by $L_{s s}=0$ [Equation (2)] are $V<V_{Z T}$ (the critical voltage of zero barrier layer thickness), and $p H<$ $p H_{\text {depass }}$ (acid depassivation). In this case, the $V_{Z T}$ and $p H_{\text {depass }}$ can be attained by extrapolating the barrier layer thickness to zero as the voltage is made less positive and the acidity of the solution becomes greater. The $V_{Z T}$ for a given $p H$, and the $p H$ at which the thickness is zero for a given voltage, $p H_{\text {depass }}$, within the acid depassivation region, are obtained from Equation (2) as

$$
V_{Z T}=\left[\left(2.303 n-4 \alpha_{2} \gamma \cdot \beta\right) \cdot p H+\ln \left(k_{2}^{0} / k_{5}^{0}\right)\right] /\left[4 a_{2}(\alpha-1) \cdot \gamma\right]
$$


and

$$
p H_{\text {depass }}=\left[4 \alpha_{2}(\alpha-1) \cdot \gamma \cdot V-\ln \left(k_{2}^{0} / k_{5}^{0}\right)\right] /\left(2.303 n-4 \alpha_{2} \gamma \cdot \beta\right)
$$

respectively. The importance of Equation (2) is therefore evident as it describes the conditions that must be met for the existence of passivity on zirconium in high temperature de-aerated aqueous solutions.

The conditions specified above may be used to develop "kinetic stability diagrams (KSDs)" as alternatives to the classical Pourbaix diagrams, noting that KSDs are kinetic descriptions of the passive state and hence are not encumbered by the need for the system to be at electrochemical equilibrium; a condition that never exists in the passive state, as demonstrated earlier in this paper. Further work is being done.

\section{Issues/Concerns: None.}

\section{References:}

1. D. D. Macdonald, J. Electrochem. Soc. 139: (1992) 3435.

2. K.S. Pitzer, Thermodynamics, McGraw-Hill, Inc., NewYork, (1995) 411.

3. D.D. Macdonald, Pure Appl. Chem., 71: (1999) 951.

4. Marc Vankeerberghen, Digby D. Macdonald, Corrosion Science, 44: (2002) 1425.

5. C.Y. Chao, L. F. Lin, and D. D. Macdonald. J. Electrochem. Soc., 129(9): (1982) 1874.

6. D. D. Macdonald and S. I. Smedley. Electrochim. Acta, 35(11/12): (1990) 1949.

7. Macdonald, D.D., M. Urquidi-Macdonald, and S. Real, J. Electrochem. Soc., 134: (1987) C419.

8. Macdonald, D.D. and M. Urquidi-Macdonald, J. Electrochem. Soc., 137: (1990) 515.

9.Urquidi-Macdonald, M., S. Real, and D.D. Macdonald, J. Electrochem. Soc., 133: (1986) 2018.

10.Urquidi-Macdonald, M., S. Real, and D.D. Macdonald, Electrochim. Acta, 35: (1990) 1559.

11.Urquidi-Macdonald, M., S. Real, and D.D. Macdonald, J. Electrochem. Soc., 133: (1986) C132.

12. D.D. Macdonald, Adan Sun, Namal Priyantha, et al., J. Electroanalytical Chem., 572: (2004) 421.

13. I. Betova, M. Bojinov, T. Latinen, et al., Corros. Sci., 44: (2002) 2675.

14. Osvaldo Pensado, Jose R. Flores, M. Urquidi-Macdonald, and D.D. Macdonald, $J$. Electrochem. Soc., 146: (1999) 1326.

15. D.D. Macdonald, S.R. Biaggio, and H. Song, J. Electrochem. Soc., 139: (1992) 170.

16. P.S. Manning, J.D. Sirman, R.A. De Souze and J.A. Kilner, Solid State Ionics, 100: (1997) 1.

17. H. Solmon, "Autodiffusion de l'oxygene, du Zirconium et de l'yttrium dans la Zircone Cubique Stabilisee par l'yttrium”, Ph.D. Thesis, Universite Paris. (1992).

18. M. de Ridder, R.G. van Welzenis, H.H. Brongersma and U. Kreissig, Solid State Ionics, 158: (2003) 67.

19. M. Bojinov, L. Hansson-Lyyra, P. Kinnunen, T. Saario, and P. Sirki, J. ASTM International, 2: (2005) 1.

20. D.D. Macdonald, J. Electrochem. Soc., in press, (2006).

21. D.D. Macdonald, ECS Transactions, (210 ${ }^{\text {th }}$ ECS Denver Meeting ) F318, in press, (2006). 


\section{Task 3. Incorporation of the PDM into the MPM}

Objectives: This task will incorporate the modified PDM into the mixed potential model (MPM), in order that the oxidation of Zircaloy under open circuit conditions can be described. This will require the development of models to describe the occurrence of cathodic reactions on Zircaloy. These models must recognize the participation of SPPs in the oxide film on charge transfer reactions, such as oxygen reduction and hydrogen evolution that occur at the bl/ol interface within the pores of the outer layer. The kinetics of charge transfer are expected to be sensitive to the number density of the SPPs, because charge transfer occurs only through a continuous electronically-conducting phase (as in the case of intermetallic SPPs that project through the oxide film). This path may involve particle-to-particle quantum mechanical tunneling of charge carriers, if the intermetallics are discontinuous through the thickness of the barrier layer. In fact, the problem of describing charge transfer is a classical percolation problem, modified by quantum effects. We have previously formulated such a model for describing electronic conduction in surge arresting materials (i.e. varistors). We propose to use a similar model in the present work to relate the exchange current densities of the cathodic reactions to the distribution of SPPs in the barrier layer, and hence ultimately to the heat treatment of the alloy. The importance of accurately modeling the cathodic processes results from the fact that they, to a large extent, control the corrosion potential, which in turn controls the thickness of the barrier oxide layer.

Task Status: The basic algorithm has been developed and is being tested on Type 304 SS in only high temperature aqueous solutions due to the lack of kinetic data for zirconium and Zircaloys. Once the required kinetic data becomes available, the algorithm for the MPM can be used to simulate zirconium and Zircaloys by entering appropriate values for the multipliers of the exchange current densities for the redox reactions and the electro-oxidation of the substrate.

The MPM is based on the physical condition that charge must be conserved in the system. Electrochemical reactions, including redox reactions or the electro-oxidation of the substrate, transfer charge across a metal/solution interface at a rate measured by the partial current. The MPM requires the sum of the partial current densities due to various charge transfer reactions to equal zero, which can be stated as:

$$
\sum_{j=1}^{n} i_{R / O, j}(E)+i_{\text {corr }}(E)=0
$$

where $i_{R / O, j}$ is the partial current density due to the $j$-th redox couple in the system; and $i_{\text {corr }}$ is the corrosion current density of the substrate. The currents are written as functions of the potential, $E$, to emphasize that partial currents depend on the potential drop across the metal/solution interface. In fact, the solution to Equation (3-1) provides the corrosion potential (ECP) being sought. Note that in deriving Equation (3-1), it is assumed the metal surface is equally accessible to all reactions in the system. 
The current density for a redox couple $\left(i_{R / O}\right)$ (Reaction (3-2)) may be expressed in terms of a general Butler-Volmer equation from electrochemical kinetics, such as Reaction (3-3).

$$
\begin{aligned}
& R \leftrightarrows O+n e \\
& i_{R / O}=\frac{e^{\left(E-E_{R / O}^{\theta}\right) / b_{a}}-e^{-\left(E-E_{R / O}^{\theta}\right) / b_{c}}}{\frac{1}{i_{0, R / O}}+\frac{1}{i_{1, f}} e^{\left(E-E_{R / O}^{\theta}\right) / b_{a}}-\frac{1}{i_{1, r}} e^{-\left(E-E_{R / O}^{\theta}\right) / b_{c}}}
\end{aligned}
$$

where $i_{0, R / O}$ is the exchange current density; $i_{1, f}$ and $i_{1, r}$ are the mass-transfer limited currents for the forward and reverse directions of Reaction (3-2), respectively; $E^{\theta}$ is the equilibrium potential for this reaction as computed from the Nernst equation; and $b_{a}$ and $b_{c}$ are the anodic and cathodic Tafel constants. Limiting currents were calculated using the following equation:

$$
i_{1, R / O}= \pm 0.0165 \cdot n \cdot F \cdot D \cdot C_{O / R}^{b} \cdot \mathrm{Re}^{0.86} \cdot S c^{0.33} / d
$$

where the sign depends on whether the reaction is in the forward $(+)$ or reverse $(-)$ direction; $C_{O / R}^{b}$ is the bulk concentration of $\mathrm{O}$ or $\mathrm{R} ; \operatorname{Re}$ is Reynolds number $(\operatorname{Re}=V d / \mathrm{v})$; $S c$ is the Schmidt number $(S c=v / D)$; $d$ is the channel diameter; $V$ is the flow velocity; and $v$ is the kinematic viscosity. Reaction (3-4) was derived from the hydrodynamic correlation for turbulent flow through a pipe, as reported by Selman and Tobias [1]. Diffusivity data for hydrogen and oxygen in high temperature aqueous systems and kinematic viscosity data for water at temperatures up to $300^{\circ} \mathrm{C}$ were taken from Macdonald's previous work and are summarized in Table 3-1.

The redox reactions of interest in this study are the oxidation of $\mathrm{H}_{2}$ and the reduction of $\mathrm{O}_{2}$ and $\mathrm{H}_{2} \mathrm{O}_{2}$ :

$$
\begin{aligned}
& \mathrm{H}_{2} \leftrightarrows 2 \mathrm{H}^{+}+2 e^{-} \\
& \mathrm{O}_{2}+4 \mathrm{H}^{+}+4 e^{-} \leftrightarrows 2 \mathrm{H}_{2} \mathrm{O} \\
& \mathrm{H}_{2} \mathrm{O}_{2}+2 \mathrm{H}^{+}+2 e^{-} \leftrightarrows 2 \mathrm{H}_{2} \mathrm{O}
\end{aligned}
$$

Exchange current density data for the reduction of oxygen in high temperature aqueous solutions was taken from the work of Lee and is given in Table 3-1. The same data was assumed to hold for the reduction of hydrogen peroxide and the oxidation of hydrogen on the basis that the current is determined by quantum mechanical tunneling across the passive film on the metal surface.

Equilibrium potential was calculated from the Nernst equation:

$$
E_{O / R}^{\theta}=E_{O / R}^{0}-\frac{2.303 R T}{n F} \log \left(\frac{a_{R}}{a_{o}}\right)
$$


where $a_{R}$ and $a_{O}$ are the thermodynamic activities of $\mathrm{R}$ and $\mathrm{O}$, respectively. The standard potential, $E_{O / R}^{0}$, for the $\mathrm{H}^{+} / \mathrm{H}_{2}, \mathrm{O}_{2} / \mathrm{H}_{2} \mathrm{O}$, and $\mathrm{H}_{2} \mathrm{O}_{2} / \mathrm{H}_{2} \mathrm{O}$ couples were taken from Macdonald's previous work. Tafel constants for the hydrogen electrode reaction are the same as those assumed in Reference [2]. Tafel constants for the reduction of oxygen on zirconium and the current-voltage curves for zirconium and Zircaloys in high temperature aqueous solutions are being measured by other research groups and we are waiting to incorporate that information in our calculations. The corrosion current was assumed to be independent of fluid flow velocity, which is considered to be a first-order approximation. $i_{c o r r}(E)$ is assumed to be velocity-independent. For calculation purposes, the passive current density takes the following form [3]:

$$
i_{\text {corr }}=\frac{\left[e^{\left(E-E_{0}\right) / b_{f, s s}}-e^{-\left(E-E_{0}\right) / b_{r, s s}}\right]}{\left[1 / i_{p}^{0}+X\right]}
$$

where $i_{\text {corr }}=0$ for $\mathrm{E}<\mathrm{E}_{0}$.

$$
X=\frac{e^{\left(E-E_{0}\right) / b_{f, s s}}}{i_{p}^{0} \exp \left[0.523\left(E-E_{0}\right)^{5}\right]}
$$

Values for the various parameters are given in Table 3-1. Equation (3-9) was developed empirically.

Table 3-1 Values for Parameters in the Mixed Potential Model

\begin{tabular}{llc}
\hline Parameter & \multicolumn{1}{c}{ Value } & Reference \\
\hline$E_{\mathrm{H}_{2}}^{0}\left(\mathrm{~V}_{\mathrm{SHE}}\right)$ & 0 & {$[2]$} \\
$E_{\mathrm{O}_{2}}^{0}\left(\mathrm{~V}_{\mathrm{SHE}}\right)$ & $1.518489-0.001121 \mathrm{~T}+6.024 \times 10^{-7} \mathrm{~T}^{2}-3.2733 \times 10^{-10} \mathrm{~T}^{3}$ & {$[2]$} \\
$E_{\mathrm{H}_{2} \mathrm{O}_{2}}^{0}\left(\mathrm{~V}_{\mathrm{SHE}}\right)$ & $1.978968-7.23 \times 10^{-4} \mathrm{~T}-9.888 \times 10^{-8} \mathrm{~T}^{2}+3.6793 \times 10^{-10} \mathrm{~T}^{3}-1.37202 \times 10^{-13} \mathrm{~T}^{4}$ & {$[2]$} \\
$\mathrm{pK}_{\mathrm{H}_{2}}$ & $-1321 / \mathrm{T}+10.703-0.010468 \mathrm{~T}$ & {$[4]$} \\
$\mathrm{pK}_{\mathrm{O}_{2}}$ & $-1202 / \mathrm{T}+9.622-0.009049 \mathrm{~T}$ & {$[4]$} \\
$\rho\left(\mathrm{g} / \mathrm{cm}^{3}\right)$ & $-4.1359+5.1788 \times 10^{-2} \mathrm{~T}-1.0911 \times 10^{-4} \mathrm{~T}^{2}+3.0193 \times 10^{-7} \mathrm{~T}^{3}-1.7809 \times 10^{-10} \mathrm{~T}^{4}$ & {$[5,6]$} \\
$\mathrm{In}\left[\mathrm{v}\left(\mathrm{cm}^{2} / \mathrm{s}\right)\right]$ & $-6.140834-1103.164 / \mathrm{T}+457155.3 / \mathrm{T}^{2}$ & {$[5,6]$} \\
$\mathrm{In}\left[\mathrm{D}_{\mathrm{H}_{2}}\left(\mathrm{~cm}^{2} / \mathrm{s}\right)\right]$ & $-5.700267-296.7439 / \mathrm{T}-288379.2 / \mathrm{T}^{2}$ & {$[5,6]$} \\
$\mathrm{D}_{\mathrm{O}_{2}}\left(\mathrm{~cm}^{2} / \mathrm{s}\right)$ & $8.03 \times 10^{-3} \mathrm{exp}(-3490 / \mathrm{RT})$ & {$[5,6]$} \\
$\mathrm{D}_{\mathrm{H}_{2} \mathrm{O}_{2}}\left(\mathrm{~cm}^{2} / \mathrm{s}\right)$ & $\mathrm{D}_{\mathrm{O}_{2}}$ & Assumed \\
$i_{H_{2}}^{0}\left(\mathrm{~A} / \mathrm{cm}^{2}\right)$ & $0.0114841 \times C_{H_{2}}^{0.5} \exp (-14244 / \mathrm{RT})$ & Assumed \\
$i_{O_{2}}^{0}\left(\mathrm{~A} / \mathrm{cm}^{2}\right)$ & $0.0114841 \times C_{O_{2}}^{0.48633} \exp (-14244 / \mathrm{RT})$ & {$[3]$} \\
$i_{\mathrm{H}_{2} \mathrm{O}_{2}}^{0}\left(\mathrm{~A} / \mathrm{cm}^{2}\right)$ & $i_{\mathrm{O}_{2}}^{0}$ & Assumed \\
$b_{f, H_{2}}(\mathrm{~V})$ & 0.065 & Assumed \\
$b_{r, H_{2}}(\mathrm{~V})$ & 0.065 & Assumed \\
$b_{f, O_{2}}(\mathrm{~V})$ & 0.071 & Assumed \\
$b_{r, O_{2}}(\mathrm{~V})$ & 0.071 & {$[3]$}
\end{tabular}




\begin{tabular}{lll}
$b_{f, H_{2} O_{2}}(\mathrm{~V})$ & $b_{f, O_{2}}$ & Assumed \\
$b_{r, H_{2} O_{2}}(\mathrm{~V})$ & $b_{r, O_{2}}$ & Assumed \\
$b_{f, Z r}(\mathrm{~V})$ & Not available now & \\
$b_{r, Z r}(\mathrm{~V})$ & Not available now & \\
$E_{0}\left(\mathrm{~V}_{\mathrm{SHE}}\right)$ & Not available now & \\
$i_{P}^{0}\left(\mathrm{~A} / \mathrm{cm}^{2}\right)$ & Not available now & \\
\hline
\end{tabular}

In view of the uncertainty in the kinetic parameters for the oxidation of hydrogen and the reduction of oxygen and hydrogen peroxide, the exchange current densities for these reactions are assumed to be adjustable parameters, so the model could be calibrated against systems for which the ECP and concentrations of electro-active species are known simultaneously. This was done by multiplying the exchange current densities for the oxidation of hydrogen and the reduction of oxygen and hydrogen peroxide by the scaling factors, $\mathrm{SF}\left(\mathrm{H}_{2}\right), \mathrm{SF}\left(\mathrm{O}_{2}\right)$, and $\mathrm{SF}\left(\mathrm{H}_{2} \mathrm{O}_{2}\right)$, respectively.

Issues/Concerns: None.

\section{References:}

[1] J.R. Selman, and C.W. Tobias, Advances in Chemical Engineering, Edited by T.B. Drew, 10 (New York, NY: Academic Press: 1978).

[2] D.D. Macdonald, and M. Urquidi-Macdonald, Corrosion 46 (1990) 380.

[3] D.D. Macdonald, Corrosion 48 (1992) 194.

[4] L.W. Niedrach, Corrosion 47 (1991) 162.

[5] D.D. Macdonald, and M. Urquidi-Macdonald, Corrosion Science 32 (1991) 51.

[6] D.D. Macdonald, J. Mankowski, M. Karaminezhaad-Ranjbar, Y-H Hu, Corrosion 44 (1988) 186.

\section{Task 4. Radiochemistry Modeling.}

Objectives: The development of robust radiolysis codes for calculating the concentrations of electroactive radiolysis products in the primary coolant circuits and, in particular, in the vicinity of the fuel in commercial BWRs and PWRs, in order to provide the link between reactor operating conditions and Zircaloy oxidation, is the objectives of this task.

Task Status: We have previously developed sophisticated radiolysis/chemistry codes for describing coolant chemistry in commercial BWRs and PWRs, and we have developed chemistry codes for commercial PWR primary coolants. The latter have now been expanded to incorporate water/solute radiolysis effects, in order to estimate the concentrations of electroactive radiolysis products around the coolant circuit and in particular at the fuel cladding/coolant interface, because it is the concentrations at this 
point that determine the ECP. Our BWR codes have been used to model fourteen operating BWRs over the past three years. The radiolysis model that we recently developed for PWRs is being modified to further emphasize radiolytic processes in the coolant at the Zircaloy/coolant interface, as well as the coolant contained within the porous outer layer of the oxide film and within CRUD deposits.

Radiation fields occur in out-of-core regions in nuclear reactors when iron and nickel-based alloys and other alloy components in contact with the primary recirculation fluid corrode, and ${ }^{59} \mathrm{Co}$ is transported into the core where it is subsequently transformed via neutron irradiation into ${ }^{60} \mathrm{Co}$, which is radioactive $(\gamma$-emitter). As the result of coolant chemistry changes, ${ }^{60} \mathrm{Co}$ is then transported throughout the heat transport circuit where it is incorporated into the corrosion product layer of magnetite, which forms on the internal metallic (carbon steel) pipe surfaces in out-of-core areas. We believe that the electrochemical potential (ECP) plays a very important role in the dissolution/precipitation of the metal in the circulating water and hence in the trapping of radioactive species in the passive films on out-of-core components.

The present codes are now sufficiently sophisticated to provide accurate simulations of the conditions that exist within the primary coolant circuits of both BWRs and PWRs and hence we regard the goals of this task as having been achieved.

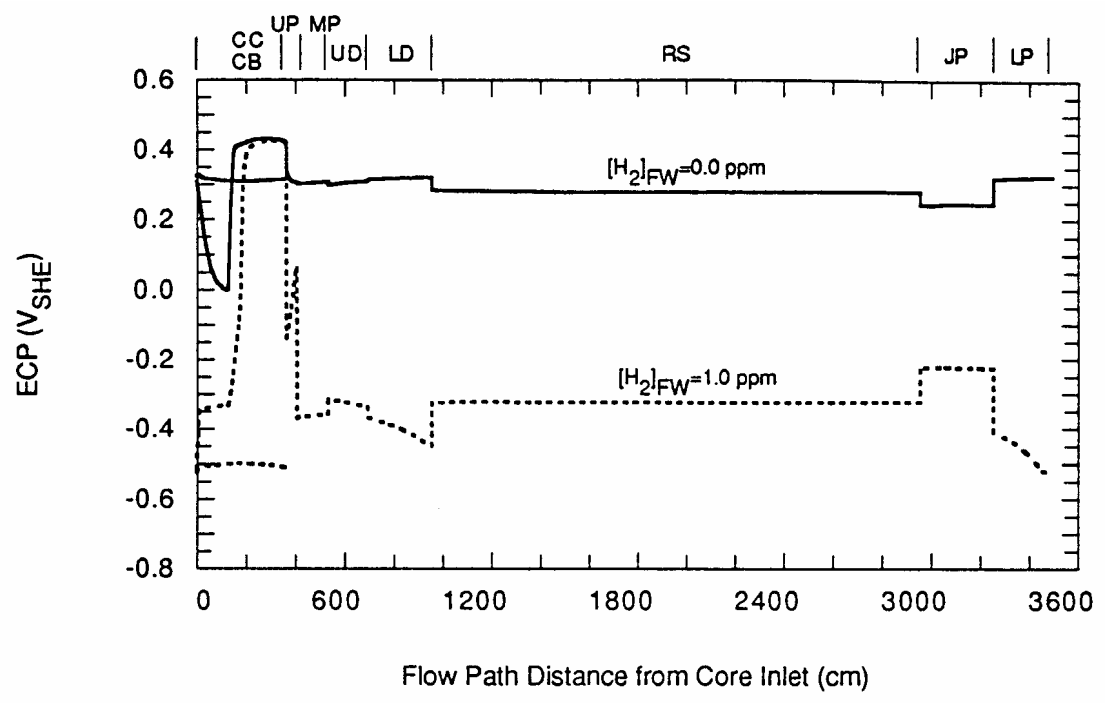

Figure 4-1: Variation of ECP around the heat transport circuit of a BWR employing general catalysis (exchange current density multiplier 104) under NWC $\left(\left[\mathrm{H}_{2}\right] \mathrm{FW}=0.0 \mathrm{ppm}\right)$ and $\mathrm{HWC}\left(\left[\mathrm{H}_{2}\right] \mathrm{FW}=1.0 \mathrm{ppm}\right)$ conditions. Note that the multiplier employed in the calculation is considered to be extreme and probably could not be achieved with deposited noble metal coatings. The value is used for illustrative purposes only.

Issues/Concerns: None. 


\section{Task 5. Modeling Growth Stresses.}

Objectives: Modeling the evolution of growth stresses within the growing oxide film, taking into account creep of the substrate, is necessary in order to estimate the time of mechanical oxide rupture (i.e. transition in growth kinetics or the onset of nodular attack). Growth stresses arise from the fact that the generation of oxygen vacancies at the $\mathrm{m} / \mathrm{bl}$ interface (Reaction 2, Figure 3) generates excess volume (due to the vacancies, themselves) that is not compensated for by other processes at this interface (e.g. annihilation of cation vacancies, Reaction 1, Figure 3). Thus the rate of generation of excess volume, and hence the rate of generation of stress (related to the cube root of the excess volume), can be related to the fluxes of the vacancies across the barrier layer. However, stress relaxation also occurs via plastic deformation of the substrate, which must be included to obtain an accurate description of the mechanical properties of oxide films.

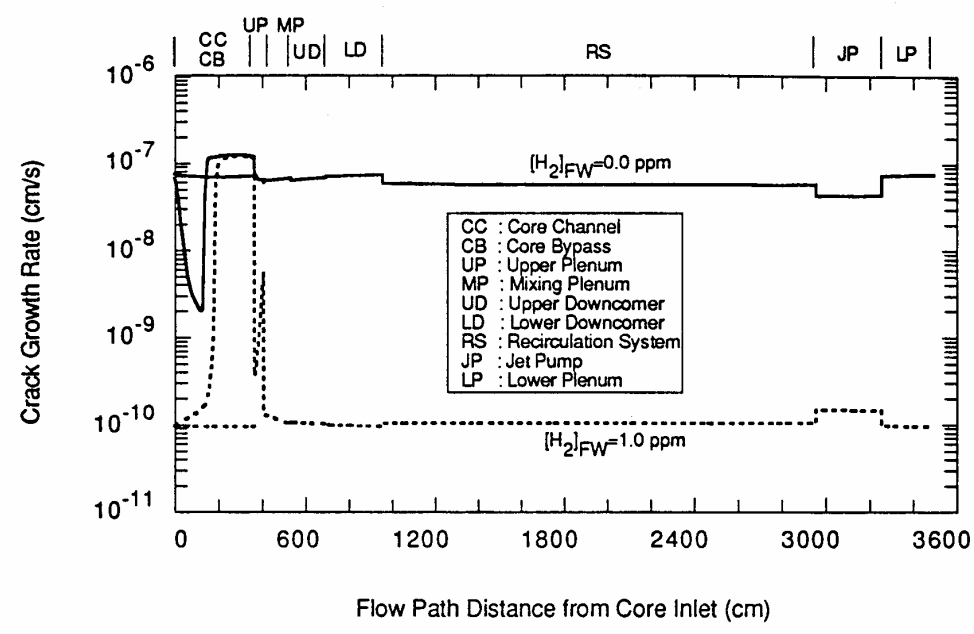

Figure 5-1: The relationship between the rate of generation of stress and the fluxes of the vacancies across the barrier layer

Task Status: No progress to report.

Issues/Concerns: None.

\section{Task 6. Experimental Measurement of Model Parameters.}

This sixth task involves the experimental measurement of critically important model parameters. The data will also be used to characterize the crystallographic and electronic defect structures of the passive film. 
Task Status: The apparatus shown in Figure 6-1 has been redesigned and rebuilt to extend the upper operating temperature from $290^{\circ} \mathrm{C}$ (the practical upper limit for Teflon) to $340^{\circ} \mathrm{C}$, which is the actual operating temperature in the core of a Pressurized Water Reactors. As shown by the flow loop in Figure 6-2, the apparatus provides for careful control of the hydrodynamic and mass transport properties of the system, while allowing for tight control over the electrochemistry of the specimen. By using very high mass transfer rates, the region of potential over which charge transfer effects can be studied is greatly expended, thereby providing for more accurate parameter determination. We have successfully carried out electrochemical experiments to study the oxidation of hydrogen on platinized nickel at $300^{\circ} \mathrm{C}$. Figure 6-3 shows typical potentiodynamic polarization curves for the oxidation of hydrogen at a hydrogen concentration of $1.4 \mathrm{ppm}$ and for a voltage step rate of $50 \mathrm{mV} / 20 \mathrm{~s}$ as a function of the rotational velocity of the flow activation impeller. The broken line corresponds to the prediction of the PDM, assuming that charge transfer is due to direct quantum mechanical tunneling across a PtO barrier layer.

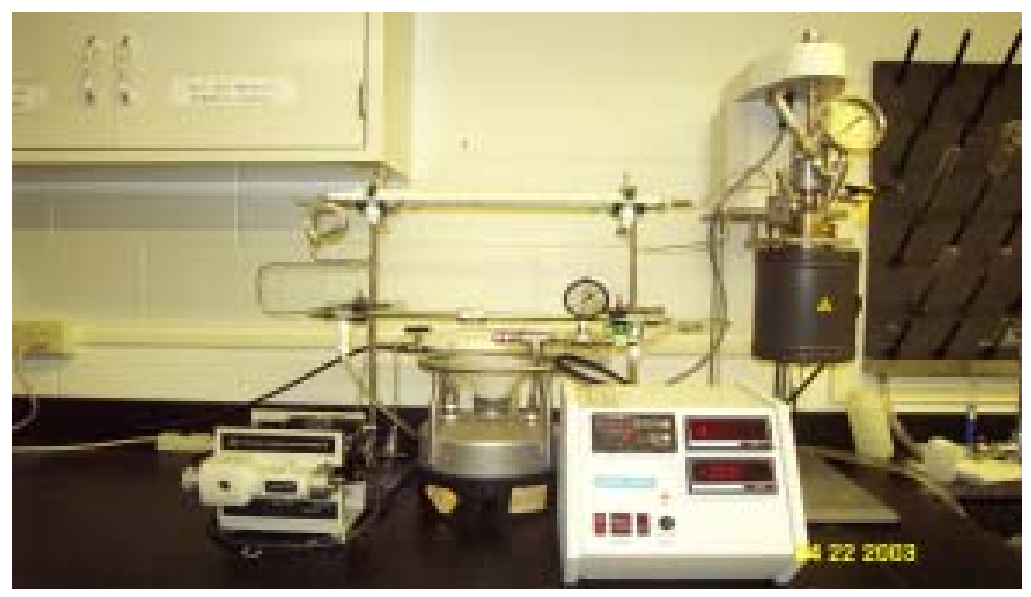

Figure 6-1. Picture of the high-temperature/high-pressure controlled hydrodynamic test system used for the electrochemical experiments. The system is designed to operate at temperatures to $300^{\circ} \mathrm{C}$ and at pressures in excess of 100b. However, due to the limitations of PTFE (Poly Tetra Fluoro Ethylene) and the need to carry out long term experiments, the maximum operating temperature is currently restricted to $250^{\circ} \mathrm{C}$. 


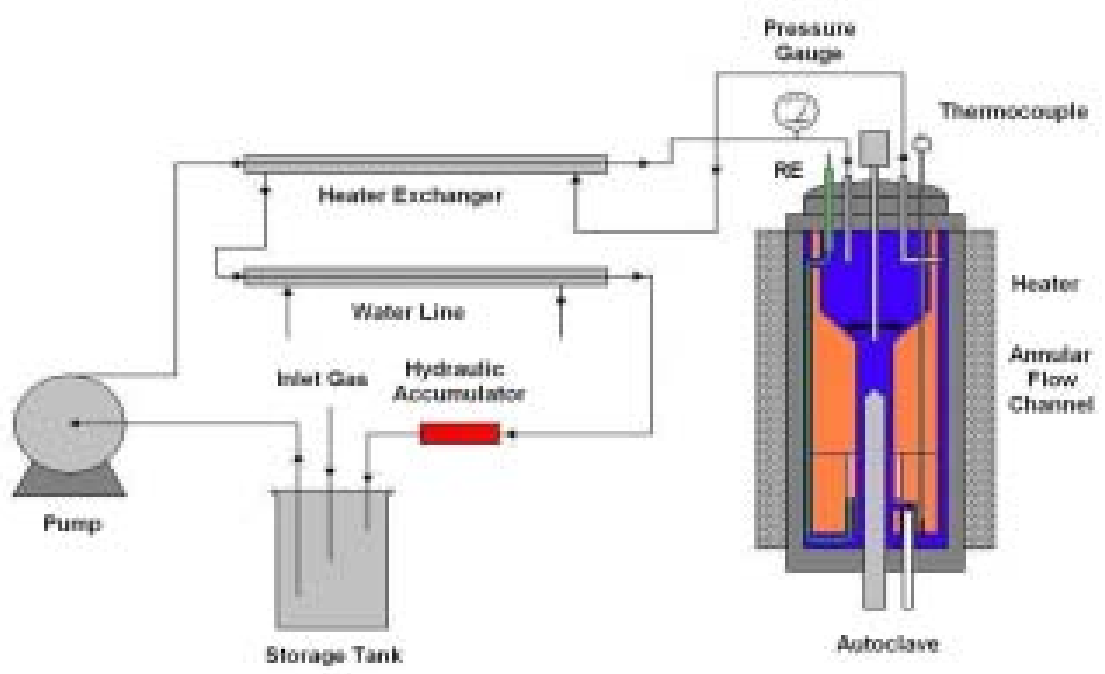

Figure 6-2. Schematic of the once-through recirculating flow loop and the controlled hydrodynamic, high-temperature/high-pressure test cell.

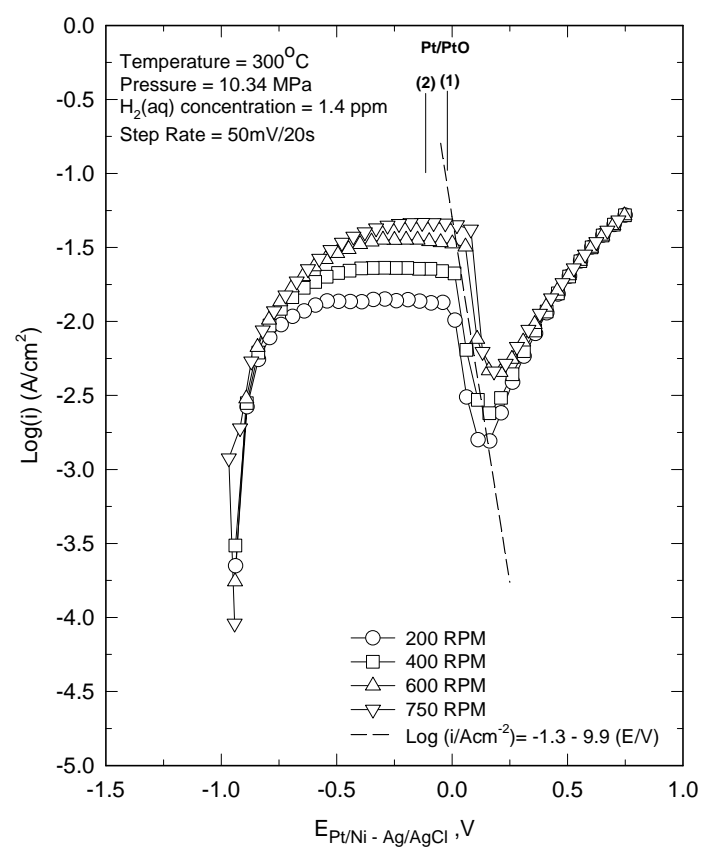

Figure 6-3. Potentiodynamic polarization curves for platinized nickel at $300^{\circ} \mathrm{C}$ in a $0.1 \mathrm{M} \mathrm{NaOH}$ solution containing $1.4 \mathrm{ppm}$ of hydrogen as measured using a positive going voltage step rate of $50 \mathrm{mV} / 20 \mathrm{~s}$, as a function of the rotational velocity of the flow activating impeller. The broken line corresponds to the prediction of the PDM combined with quantum mechanical tunneling theory. 
Researchers have completed the characterization of passive film formed on pure zirconium under simulated BWR and PWR primary coolant conditions at temperatures up to $300^{\circ} \mathrm{C}$, including polarization scans, impedance measurements, Mott-Schottky analysis, current transients, thickness transients, etc. Based on these measurements, electronic structure, donor density, effect of potentials on film formation rate and steady state thickness, dissolution rates, and other properties of zirconium oxide films have been determined. The relationship of the second phase particles and the properties of the passive films formed on the different heat treated Zircaloy-4 samples are being examined by using the controlled hydrodynamic apparatus at $250^{\circ} \mathrm{C}$ in the hydrogenated environments.

The three-electrode test cell comprised a $600 \mathrm{~mL}$ reactor containing a magnetically activated impeller, a thermocouple, a working electrode, a counter electrode, a reference electrode, and an annular flow channel. The working electrode was fabricated from polycrystalline zirconium (99.2\%) rod from Alfa Aesar and had an exposed surface area of $5.82 \mathrm{~cm}^{2}$. Zirconium rod used in this study contains $0.25 \mathrm{wt} \% \mathrm{Sn}, 0.01 \mathrm{wt} \% \mathrm{Ni}$, $0.11 \mathrm{wt} \% \mathrm{Fe}$, and $0.01 \mathrm{wt} \% \mathrm{Cr}$, determined by Electron Probe Micro Analysis (EPMA). The sample was abraded with 800 and 1200 grit SiC paper, and then further polished with $3 \mu \mathrm{m}$ and $1 \mu \mathrm{m}$ diamond paste one day before the experiment. An insulated $316 \mathrm{~L}$ wire was connected to the bottom of the annular flow channel by a small screw and then taken out through the top of the vessel via an insulated pressure fitting, with the whole channel serving as the counter electrode. The flow channel was also used to direct the flowing solution over the specimen and to establish well-developed hydrodynamic and mass transport regimes. Previous studies on an identical channel demonstrated that linear flow velocities of several meters per second could be achieved at $300^{\circ} \mathrm{C}$.

Two kinds of reference electrode were used in this study; a $\mathrm{Ag} / \mathrm{AgCl}$ (sat. $\mathrm{KCl}$ ) and $\mathrm{Pt} / \mathrm{H}_{2}$ for use in non-hydrogenated and hydrogenated environments, respectively. The $\mathrm{Ag} / \mathrm{AgCl}$ (sat. $\mathrm{KCl}$ ) reference electrode comprised a silver rod that had been plated with silver chloride in a $0.1 \mathrm{M} \mathrm{HCl}$ solution at a current density of $5 \mathrm{~mA} / \mathrm{cm}^{2}$ for 18 hours. The $\mathrm{Ag} / \mathrm{AgCl}$ rod was housed in a PTFE tube fitted with a porous zirconia plug at one end with the silver rod protruding through a PTFE cylinder. The inner compartment was filled with $\mathrm{KCl}$ crystals and saturated $\mathrm{KCl}$ solution with sufficient solid $\mathrm{KCl}$ to ensure that the solution remained saturated at all temperatures. The stability of the reference electrode at ambient temperature was checked against a Saturated Calomel Electrode (SCE) before and after experiments. The porous zirconia liquid junction of the reference electrode was guided to a hole that had been machined from the bottom of the channel to a point just opposite of the specimen (Figure 6-2), in order to minimize the uncompensated resistance that would be experienced in the electrochemical studies. For consistency in reporting electrochemical potentials, all measured potentials were converted to the Standard Hydrogen Electrode (SHE) scale by thermodynamic calculation.

To simulate the PWR primary water chemistry, aqueous solutions containing $0.1 \mathrm{M} \mathrm{B}(\mathrm{OH})_{3}+0.001 \mathrm{M} \mathrm{LiOH}$ were used. Pressurized water reactors operate with boric acid as a nuclear shim to control the nuclear reactivity. Lithium hydroxide ( $\mathrm{LiOH})$ is produced by the nuclear reaction ${ }_{5} \mathrm{~B}^{10}+{ }_{0} \mathrm{n}^{1} \rightarrow{ }_{3} \mathrm{Li}^{7}+{ }_{2} \mathrm{He}^{4}$, but the total lithium 
concentration is controlled in concert with the boric acid concentration in order to minimize the release of corrosion products into the coolant and to inhibit their deposition onto the fuel surfaces. Typically, a hydrogen concentration $25-35 \mathrm{cc}(\mathrm{STP}) / \mathrm{kg}\left(\mathrm{H}_{2} \mathrm{O}\right)$ is maintained to inhibit the formation of radiolytic oxygen in the coolant. In our study, different hydrogen concentrations have been tried to test the influence on zirconium passive film formation. The hydrogen content in the solution was adjusted by bubbling ultra-high purity hydrogen gas at an appropriate pressure through the solution in the storage tank overnight at room temperature before the solution was continuously fed into the autoclave. The solutions were prepared from boric acid (Alfa Aesar), lithium hydrogen (Merck), and deionized water (milli-Q system, $18.2 \mathrm{M} \Omega \mathrm{cm}^{-1}$ ). All experiments reported here were carried out at $250^{\circ} \mathrm{C}$ and at a pressure of $62 \mathrm{bar}(900 \mathrm{psi})$ in order to maintain a single liquid phase in the system. The lower temperature than that found in reactor coolant circuits $\left(288^{\circ} \mathrm{C}\right.$ for BWRs and $360^{\circ} \mathrm{C}$ for PWRs) was dictated by the need to use PTFE in the electrochemical cell and the use of the boric acid/lithium hydroxide for the BWR case was dictated by the need to maintain constant $\mathrm{pH}$. The simulated BWR coolant comprised $0.1 \mathrm{M} \mathrm{B}(\mathrm{OH})_{3}+0.001 \mathrm{M} \mathrm{LiOH}$ for $\mathrm{pH}$ control, as in the PWR case, but the solution was sparged with argon, rather than with hydrogen. Of course, the coolant in the primary circuit of a BWR is nominally pure water, but electrochemical studies require a significant conductivity in order to study the properties of the system; the required conductivity was provided by the buffer.

\subsection{Electrochemistry of Zirconium}

The passive films formed anodically on zirconium in the $1 \mathrm{M} \mathrm{B}(\mathrm{OH})_{3}+0.001 \mathrm{M}$ $\mathrm{LiOH}$ solution at $250^{\circ} \mathrm{C}$ and 62 bar (900 psi) display golden, blue, purple, or black colors, depending on the formation potential and exposure time. All the films formed on the samples, polished to $1 \mu \mathrm{m}$ finish, were shiny and smooth while localized corrosion was noticed on the unpolished sample surface. All electrochemical experiments reported below were carried out on polished samples.

\section{The Electrochemical Thermodynamics of Reactor Cores}

We have employed the Point Defect Model (PDM) [1, 2], which provides an atomistic description of the growth and breakdown of oxide and hydride [3-5] films in terms of the generation and annihilation of crystallographic defects at the interfaces of the barrier layer and their transport under the influence of an electrical potential gradient within the oxide (or hydride) phase, to interpret the experimental data. As reported earlier in this report, that the PDM is being considerably modified by the incorporation of the outer, porous oxide layer and the formation of a defective hydride $\left(\mathrm{ZrH}_{2-x}\right)$ barrier layer in the case of hydrogenated solutions and a defective oxide $\left(\mathrm{ZrO}_{2-x}\right)$ layer in the case of non-hydrogenated solutions [6].

Before proceeding further, it is necessary to discuss briefly the electrochemical thermodynamics of the fuel cladding in BWRs and PWRs. In order to do so, we need to calculate the equilibrium properties of the couples $\mathrm{Zr} / \mathrm{ZrO}_{2}, \mathrm{ZrH}_{2} / \mathrm{Zr}$, and $\mathrm{ZrH}_{2} / \mathrm{ZrO}_{2}$, where the reduced species is on the left side and the oxidized species is on the right side. 
Following the Stockholm convention, we write all half cell reactions in the reduction sense as

$$
\begin{aligned}
& \mathrm{ZrO}_{2}+4 \mathrm{H}^{+}+4 e^{-}=\mathrm{Zr}+2 \mathrm{H}_{2} \mathrm{O} \\
& \mathrm{Zr}+2 \mathrm{H}^{+}+2 e^{-}=\mathrm{ZrH}_{2}
\end{aligned}
$$

and

$$
\mathrm{ZrO}_{2}+6 \mathrm{H}^{+}+6 e^{-}=\mathrm{ZrH}_{2}+2 \mathrm{H}_{2} \mathrm{O}
$$

to yield the cell reactions as

$$
\begin{aligned}
& \mathrm{ZrO}_{2}+2 \mathrm{H}_{2}=\mathrm{Zr}+2 \mathrm{H}_{2} \mathrm{O} \\
& \mathrm{Zr}+\mathrm{H}_{2}=\mathrm{ZrH}_{2}
\end{aligned}
$$

and

$$
\mathrm{ZrO}_{2}+3 \mathrm{H}_{2}=\mathrm{ZrH}_{2}+2 \mathrm{H}_{2} \mathrm{O}
$$

The standard potential is then given by $E^{0}=-\Delta G_{R}^{0} / n F$, where $\Delta G_{R}^{0}$ is the change in standard Gibbs energy for Reactions (6-4) to (6-6), $n$ is the number of electrons involved in the half cell reaction, and $F$ is Faraday's constant (96,487 C/equiv.). Calculation of the standard potentials at temperatures up to the maximum core temperature of the reactor type of interest was carried out using the HSC software. The half cell reactions can be written in general form as

$$
a A+x H^{+}+n e^{-}=b B+c H_{2} O
$$

from which we derive the Nernst equation as

$$
\begin{aligned}
& E=E^{0}-\frac{2.303 R T}{F}\left(\frac{b}{n}\right) \log \left(a_{B}\right)+\frac{2.303 R T}{F}\left(\frac{a}{n}\right) \log \left(a_{A}\right) \\
& -\frac{2.303 R T}{F}\left(\frac{c}{n}\right) \log \left(a_{\mathrm{H}_{2} \mathrm{O}}\right)-\frac{2.303 R T}{F}\left(\frac{x}{n}\right) p H
\end{aligned}
$$

where $a_{i}$ is the activity of species $i, R$ is the universal gas constant $(R=8.3124 \mathrm{~J} / \mathrm{K} . \mathrm{mol})$, and $p H=-\log \left(a_{H^{+}}\right)$. Because the reactants and products are solids, we may set their activities to one and because the solutions are reasonably dilute, $a_{H_{2} \mathrm{O}}=1$. Thus, Equation (6-8) reduces to the much simpler form

$$
E=E^{0}-\frac{2.303 R T}{F}\left(\frac{x}{n}\right) p H
$$


where $x$ is the stoichiometric coefficient of $\mathrm{H}^{+}$in the half cell reactions, Equations (6-1) to $(6-3)$.

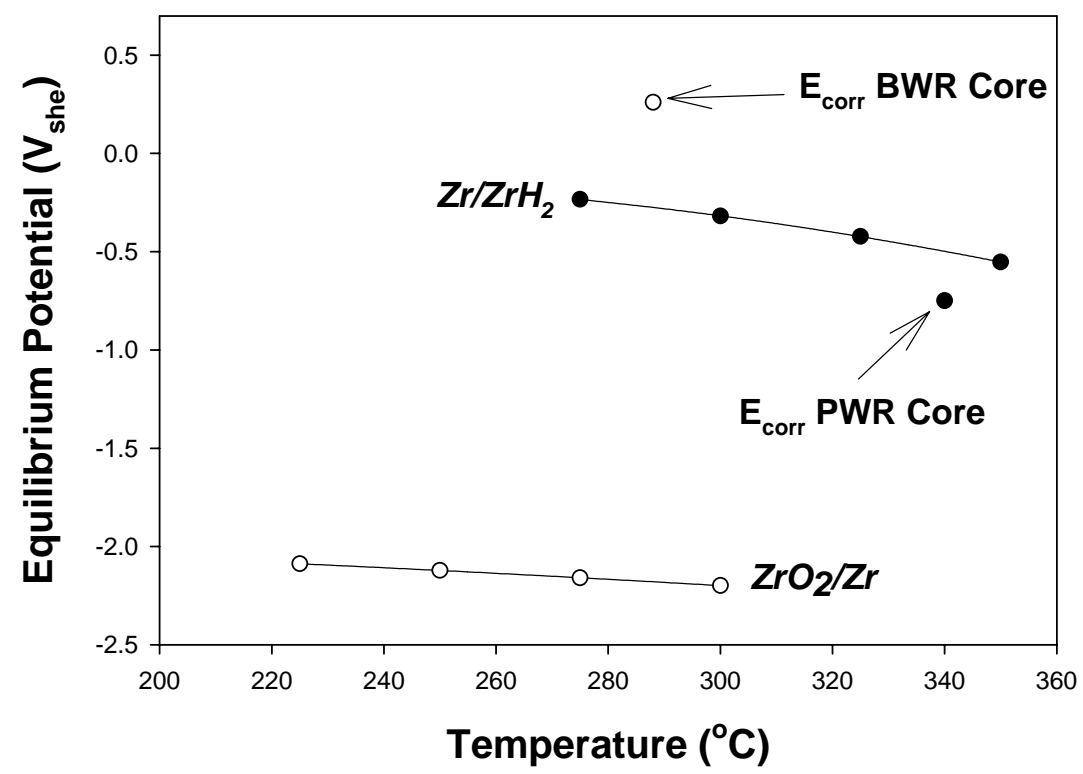

Figure 6-4: Comparison of the electrochemical conditions that exist in the cores of BWRs and PWRs in relationship with the equilibrium potentials for the $\mathrm{Zr} / \mathrm{ZrH}_{2}$ and $\mathrm{ZrO}_{2} / \mathrm{Zr}$ reactions. The equilibrium potentials for the PWR core were calculated assuming $[\mathrm{B}]=1000 \mathrm{ppm}$ and $[\mathrm{Li}]=2 \mathrm{ppm}$ while those for the BWR core assumes pure water to be the coolant. The corrosion potentials for the PWR core were taken from Urquidi-Macdonald and Macdonald [7] whereas that for the BWR core was taken from Yeh, Macdonald, and Motta [8]. After Park et al [6].

That the electrochemical conditions in the cores of BWRs and PWRs differ substantially is demonstrated by Figure 6-4, in which is plotted the equilibrium potentials for the $\mathrm{Zr} / \mathrm{ZrO}_{2}$ and $\mathrm{ZrH}_{2} / \mathrm{Zr}$ couples as a function of temperature. Also plotted are the ECP (corrosion potential) values for the fuel channels in the two types of reactors, as determined by thermohydraulic/radiolysis/electrochemical modeling in this laboratory. The reader will note that the formation of $\mathrm{ZrO}_{2}$ is an oxidation process $\left(\mathrm{Zr}+2 \mathrm{H}_{2} \mathrm{O} \rightarrow \mathrm{ZrO}_{2}+4 \mathrm{H}^{+}+4 e^{-}\right)$, whereas the formation of the hydride is a reduction process $\left(\mathrm{Zr}+2 \mathrm{H}_{2} \mathrm{O}+2 e^{-} \rightarrow \mathrm{ZrH}_{2}+2 \mathrm{OH}^{-}\right)$, with the currents being positive and negative respectively. From Pourbaix's magic formula, $\left.\left[\left(E-E^{e}\right) I \geq 0\right)\right]$, where $E$ is the potential (ECP) and $E^{e}$ is the equilibrium potential, we see that the formation of $\mathrm{ZrO}_{2}$ as the barrier layer is spontaneous in the BWR case (because $E>E^{e}$ ), whereas the formation of $\mathrm{ZrH}_{2}$ as the barrier layer is spontaneous in the PWR case (because $E<E^{e}$ ). Of course, this argument is couched in terms of the stoichiometric phases and the exact identity of the hydride phase remains uncertain, but the differences in the ECP and the equilibrium potentials are sufficiently large (Figure 6-4) that it is unlikely that non-stoichiometry 
alone could change the Gibbs energies of formation to the extent necessary to alter these conclusions. That the thermodynamics are consistent with observation is supported by the micrographs shown in Figure 6-5. Thus, in the BWR case, only oxides are detected, but in the PWR case a continuous hydride layer, representing the barrier layer, is found to exist between the metal (which also contains discrete hydride platelets) and the outer oxide layer.

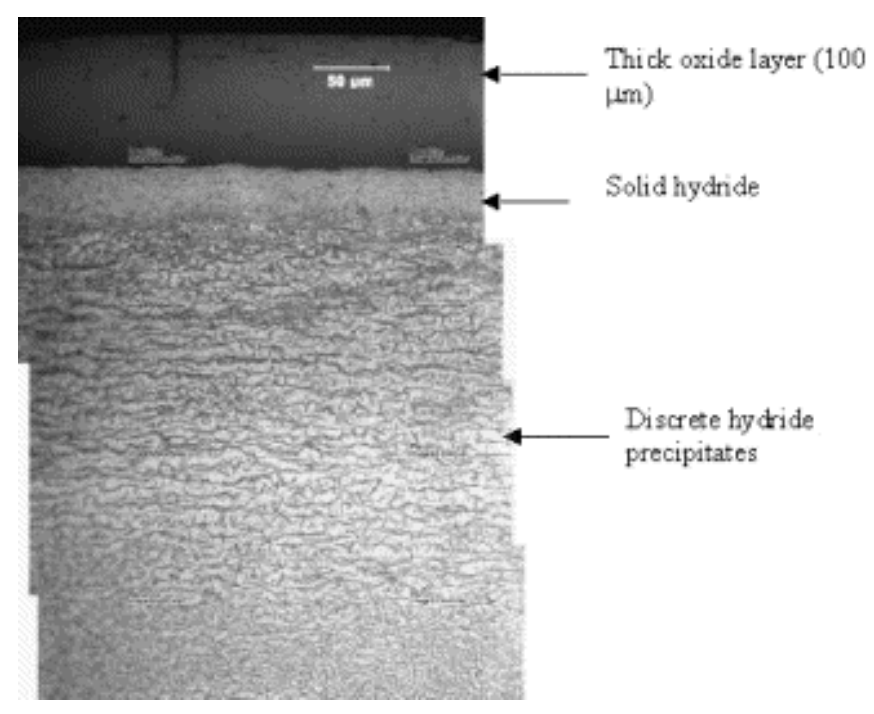

Figure 6-5: A continuous hydride layer and discrete hydride in the Zircaloy-4 cladding tube (average fuel burnup of $67 \mathrm{GWd} / \mathrm{t}$ and fast fluence of $1.3 \times 1022$ $\mathrm{n} / \mathrm{cm}^{2}$ ) [Courtesy of R. Daum, Argone National Laboratory].

Besides, the work of HKL technology [9] also shown the formation of a continuous hydride layer at the metal/film interface on various zirconium alloys under laboratory conditions. Titanium, which is very closely related to zirconium in its chemical and electrochemical properties, also forms a continuous hydride layer at the metal/oxide film interface, as shown by the work of Wang, et.al. [10] at ambient temperature. The thickness of the titanium hydride layer increases as the voltage is made more negative, as predicted by the PDM $[1,2]$. An almost continuous hydride layer was reported to form at the metal/oxide interface in Zircaloy-4 when cathodically polarized in dilute sodium sulphate solution at elevated temperature $\left(300{ }^{\circ} \mathrm{C}\right)$ [11], p122] and the cathodic polarization method was also used by Cox el. al. [32] to form a hydride layer on this alloy in dilute $\mathrm{CuSO}_{4}$ solution at the same temperature. While the existence of a solid hydride layer at the metal/oxide interface is controversial, primarily because few experiments have been carried out under well-controlled electrochemical conditions, we note that still other researchers have reported experimental evidence for hydride layer formation on Zircaloy-4 at elevated temperatures [12-14]. Furthermore, thermodynamic calculations described above indicate that, if the corrosion potential is sufficiently positive (as in a BWR core), only oxide formation is thermodynamically spontaneous, whereas, if the potential is sufficiently negative (PWR), the formation of both the hydride $\left(\mathrm{ZrH}_{2}\right)$ and the oxide is spontaneous, as noted above. To our knowledge, the interfacial 
hydride layer has not been reported in the BWR case, in keeping with the thermodynamic predictions. It is on this basis that we postulate that the barrier layer on zirconium under PWR fuel cladding conditions is the defective hydride $\left(\mathrm{ZrH}_{2-\mathrm{x}}\right)$ and that the outer layer is the porous oxide $\left(\mathrm{ZrO}_{2}\right)$, whereas in the BWR case the barrier layer is the defective oxide $\left(\mathrm{ZrO}_{2-\mathrm{x}}\right)$ and the outer layer is the porous, non-defective $\mathrm{ZrO}_{2}$. Only the barrier layer, which is a small (but important) part of the passive film, has been postulated as being a hydride. The barrier layer is typically a few tens of nanometers thick, whereas the oxide outer layer may be many microns thick depending upon the conditions, so that the great bulk of the passive film is still the oxide, even under highly reducing PWR primary coolant conditions.

\section{$\underline{\text { Polarization Studies }}$}

The potentiodynamic polarization curves for zirconium in the same solution sparged with different gases before and during experiment are different (figure 6-6). For the inert gas case, the solution reservoir at ambient temperature was sparged with highpurity argon gas, whereas for hydrogen case, ultra high purity hydrogen was used to sparge the solution overnight before continuously fed into the autoclave. The pressure of hydrogen in solution reservoir is maintained at appropriate value at room temperature during experiments, by suitably controlling the total gas pressure in the system. The polarization curves for zirconium (Figure 6-6) were recorded under potentiodynamic conditions by starting the voltage sweep at a rate $1 \mathrm{mVs}^{-1}$ from a point $0.2 \mathrm{~V}$ more negative than the open circuit potential. For the passive film formed in the solution containing $22 \mathrm{~cm}^{3}(\mathrm{STP}) / \mathrm{kg}\left(\mathrm{H}_{2} \mathrm{O}\right)$ hydrogen, the passive region extends from $-0.2 \mathrm{~V}_{\text {she }}$ to around $0.5 \mathrm{~V}_{\text {she; }}$ at higher potentials, the system exhibits transpassive behavior as indicated by a steeper rise in the current density. The polarization curve for zirconium in the same solution but without hydrogen displays a much more positive zero current potential with the available passive region extending from $0 \mathrm{~V}_{\text {she }}$ to $0.7 \mathrm{~V}_{\text {she. As shown, }}$ the zero current potential of zirconium is lowered by more than $0.45 \mathrm{~V}$ by the introduction of $22 \mathrm{~cm}^{3}(\mathrm{STP}) / \mathrm{kg}\left(\mathrm{H}_{2} \mathrm{O}\right)$ hydrogen into the solution.

Potentiostatic experiments were carried out by holding the potential at the desired value for film growth until there was no perceptible change in current over 24 hours. The logarithm of the stationary current density for the passive film formed on zirconium in hydrogen-free or hydrogenated solution is found to be independent of the formation potential (Fig. 6-7). The stationary current density is slightly greater for the former than for the latter, indicating that the oxidation of hydrogen contributes little to the observed current. The independence of the logarithm of the current density on the applied potential is consistent with the diagnostic criteria of PDM [15] for an n-type passive film. The n-type electronic character of the passive film is further demonstrated by the capacitance measurement described later in this report. 


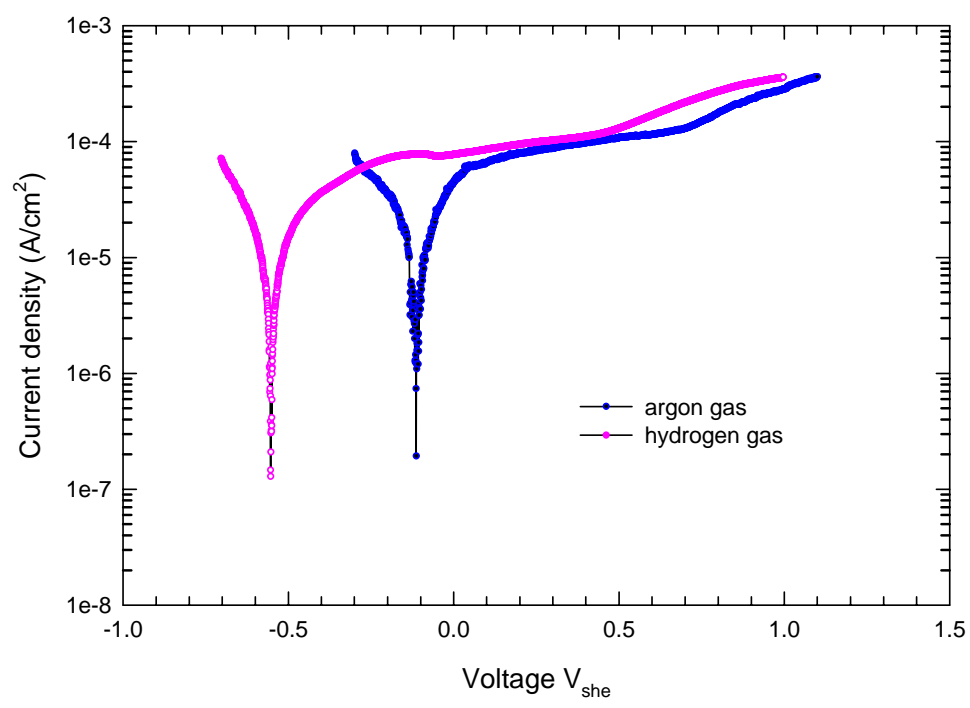

Figure 6-6: Comparison of polarization curves for $\mathrm{Zr}$ in the hydrogenated and hydrogen-free environments. The electrolyte was $0.1 \mathrm{M} \mathrm{B}(\mathrm{OH})_{3}+0.001$ $\mathrm{M} \mathrm{LiOH}$, with the solution reservoir at ambient temperature being sparged with high-purity argon gas or hydrogen gas before and during the experiments. For the hydrogen case, $22 \mathrm{~cm}^{3}(\mathrm{STP}) / \mathrm{kg}\left(\mathrm{H}_{2} \mathrm{O}\right)$ hydrogen was maintained in the reservoir by suitably controlling the total gas pressure in the system. Scan rate $=1.0 \mathrm{mV} / \mathrm{s}, \mathrm{T}=250^{\circ} \mathrm{C}$ and $\mathrm{P}=62$ bar.

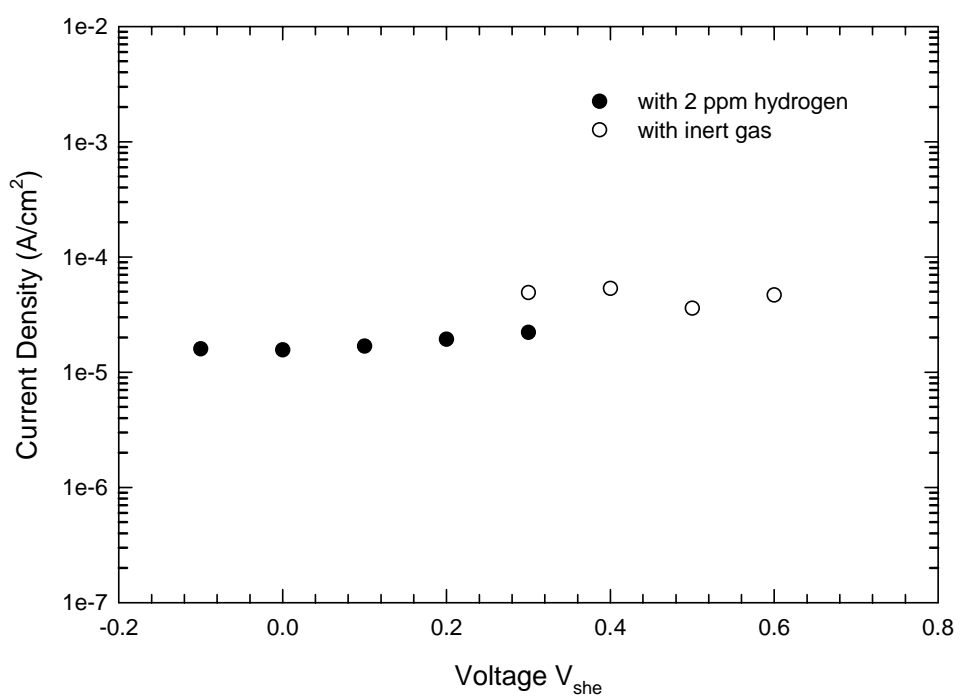

Figure 6-7: Dependence of the steady state current density on applied potentials for passive film formed in pure zirconium in $0.1 \mathrm{M} \mathrm{B}(\mathrm{OH})_{3}+0.001$ $\mathrm{M} \mathrm{LiOH}$ solution with or without hydrogen. The potentials were changed in the negative- to-positive direction. The current data reported here was the value after holding 24 hours at each potential step for film growth. $\mathrm{T}=250{ }^{\circ} \mathrm{C}$ and $\mathrm{P}=62$ bar. 
The film thickness was calculated from the measured capacitance. For capacitance measured at frequencies higher than $1 \mathrm{kHz}$, the electrochemical impedance displays an almost purely capacitive frequency response. Accordingly, we use the wellknown "parallel plate" expression for the capacitance to estimate the steady state film thickness, $\mathrm{L}_{\mathrm{ss}}$

$$
L_{s s}=\frac{\varepsilon \varepsilon_{0} A}{C}
$$

where $A$ is the area, $\varepsilon_{0}$ is the vacuum permittivity $\left(8.85 \times 10^{-14} \mathrm{~F} / \mathrm{cm}\right)$ and $\varepsilon=22[11]$ is the dielectric constant of the passive film. In Figure 6-8, the capacitance, $\mathrm{C}_{\mathrm{p}}$, is presented as a function of the applied potential $(\mathrm{E}) . \mathrm{C}_{\mathrm{p}}$ exhibits a considerable potential dependence, and it is found to vary with potential in a nearly parabolic manner (Figure 6-8). Such a parabolic dependence is to be expected, if $\mathrm{C}_{\mathrm{p}}$ represents the space-charge capacity $\left(\mathrm{C}_{\mathrm{sc}}\right)$. As shown in Figure 6-9, a linear plot of $\mathrm{C}_{\mathrm{p}}^{-1}$ vs. potential is found, indicating that the thickness $\left(\mathrm{L}_{\mathrm{ss}}\right)$ of the passive film increases linearly with the applied potential (see Figure 6-10). The film thickness (Figure 6-10) is found to be linear function of the applied potential. The anodizing constants are $121 \mathrm{~nm} / \mathrm{V}$ for the passive film formed on zirconium in the hydrogenated solution and $79 \mathrm{~nm} / \mathrm{V}$ for that formed in the solution without hydrogen. These values are much greater than the $1.9-2.5 \mathrm{~nm} / \mathrm{V}$ normally found for barrier layer formed on metals and alloys at ambient or near-ambient temperatures. The higher values are attributed to the formation of thick outer layers over thin barrier layers, such that the measured capacitance, which may be represented as a series combination of the two layers, is dominated by the outer layer, as shown by the following equation

$$
\frac{1}{C}=\frac{1}{A \varepsilon_{0}}\left[\frac{L_{b l}}{\varepsilon_{b l}}+\frac{L_{o l}}{\varepsilon_{o l}}\right]
$$

Thus, noting that the dielectric constants for the barrier layer and the outer layer are probably of similar magnitude (this is certainly the case for BWR conditions, where both layers comprise $\mathrm{ZrO}_{2}$ ), then for $L_{o l}>L_{b l}$ the measured capacitance is dominated by the outer layer. Since the outer layers in both environments is porous $\mathrm{ZrO}_{2}$, the data plotted in Figure 6-10 indicate that the outer layer is significantly thicker when formed in the hydrogenated solution than when grown in the hydrogen-free solution. This finding also accounts for the difference in the passive current density, also shown in Figure 6-7, because of the expected impact of the outer layer on the interfacial impedance. Finally, Equation (6-11) should be regarded as being a first approximation, because the barrier layer also possesses semiconductor properties that can be interpreted in terms of MottSchottky theory (see below). This first approximation is valid, because the outer layer dominates the capacitance. 


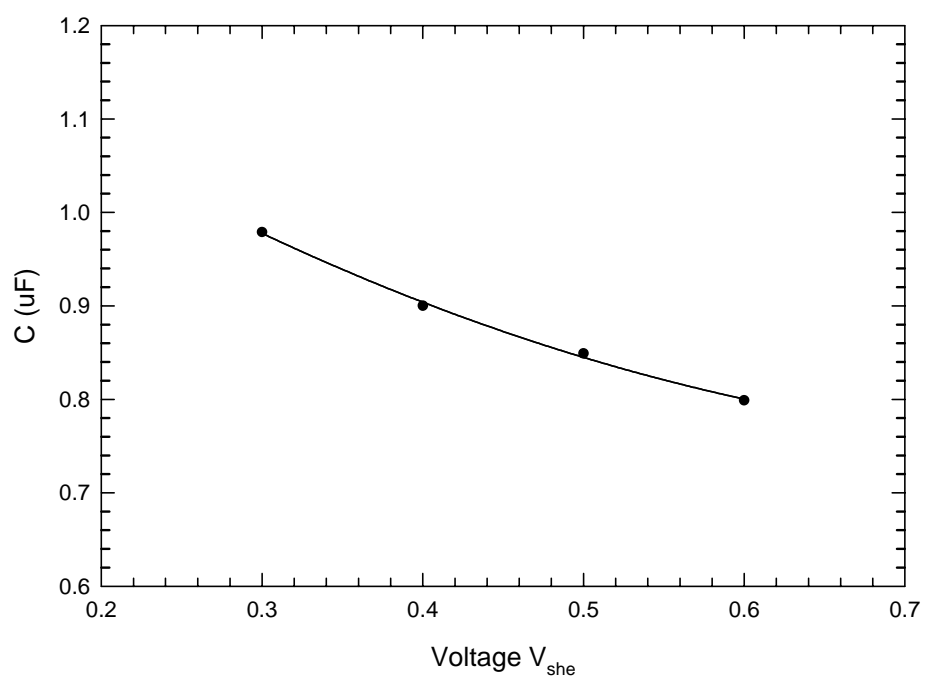

Figure 6-8. Effect of film formation potential on the capacitance of the passive film formed on zirconium in $0.1 \mathrm{M} \mathrm{B}(\mathrm{OH})_{3}+0.001 \mathrm{M} \mathrm{LiOH}$ solution without hydrogen. $\mathrm{T}=250^{\circ} \mathrm{C}$ and $\mathrm{P}=62$ bar.

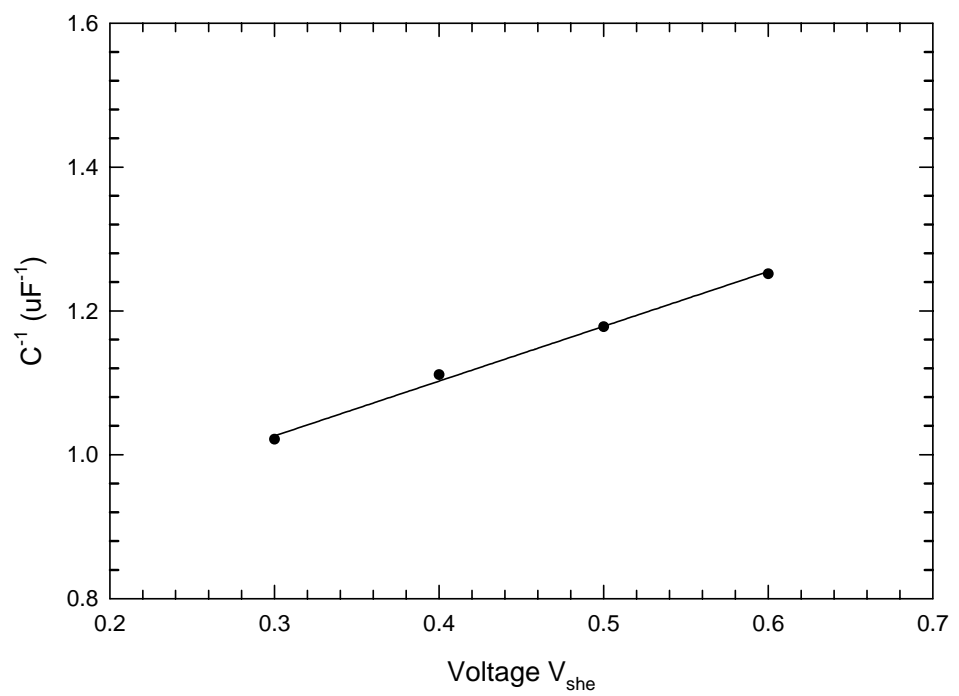

Figure 6-9: Dependence of reciprocal capacitance for passive zirconium in $0.1 \mathrm{M} \mathrm{B}(\mathrm{OH})_{3}+0.001 \mathrm{M} \mathrm{LiOH}$ solution without hydrogen measured by holding at each potential 24 hours for film growth. $\mathrm{T}=250^{\circ} \mathrm{C}$ and $\mathrm{P}=62 \mathrm{bar}$. 


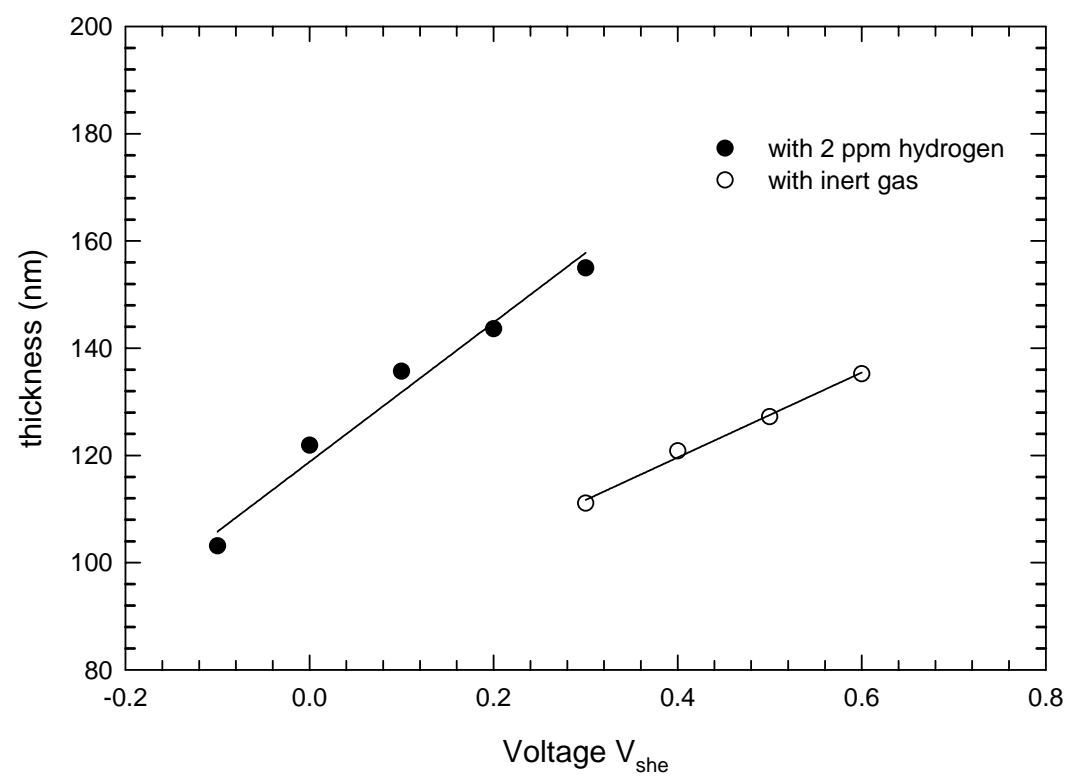

Figure 6-10: Dependence of the zirconium passive film thickness on applied potential in $0.1 \mathrm{M} \mathrm{B}(\mathrm{OH})_{3}+0.001 \mathrm{M} \mathrm{LiOH}$ solution with 22 $\mathrm{cm}^{3}(\mathrm{STP}) / \mathrm{kg}\left(\mathrm{H}_{2} \mathrm{O}\right)$ or without hydrogen. The potential was changed in the negative-to-positive direction. The film thickness was measured after holding potential at each step for 24 hours for film stabilization. $\mathrm{T}=250{ }^{\circ} \mathrm{C}$ and $\mathrm{P}=$ 62 bar.

\section{Electrochemical Impedance Spectroscopy}

In our studies, electrochemical impedance spectroscopy (EIS) is being extensively used to characterize the passive film. The EIS data are being used to distinguish between different mechanisms for the growth of the anodic oxide film on zirconium and Zircaloys and to determine kinetic parameters for various reactions in the models. The power of EIS lies in the fact that each frequency yields a separate experimental observation, so that it is possible, by measuring the impedance at many frequencies, to resolve the kinetic parameters (rate constants and transfer coefficients) for multi-step reaction mechanisms.

Electrochemical impedance spectra (EIS) for passive zirconium were measured over a wide range of frequencies (typically $100 \mathrm{kHz}$ to $0.01 \mathrm{~Hz}$ ) as a function of formation potential across the passive range in hydrogenated solution and solution without hydrogen at $250^{\circ} \mathrm{C}$. The impedance was measured after holding the potential at desired value for 24 hours, to ensure that the passive film growth arriving the steady state. The measurements were carried out in the ascending potential direction. Since the stability of the electrochemical system during EIS measurement is critical to obtaining viable data, the quality of the EIS data are checked both experimentally and theoretically. The data were checked experimentally by stepping the frequencies from high-to-low and 
then immediately back from low-to-high, with the impedance being measured at each step, to ascertain that the same values were obtained at equivalent frequencies in the two directions. If the system is in the steady state, which means the thickness and current are independent of time, the impedance data should match in the two potential step directions. The Nyquist plot and Bode plot (Figure 6-11) display perfect matches for the impedance data measured in the two step directions after holding the potential at $0.1 \mathrm{~V}_{\text {she }}$ for 24 hours for the film to attain a steady state. This is a particularly good test for system stability.
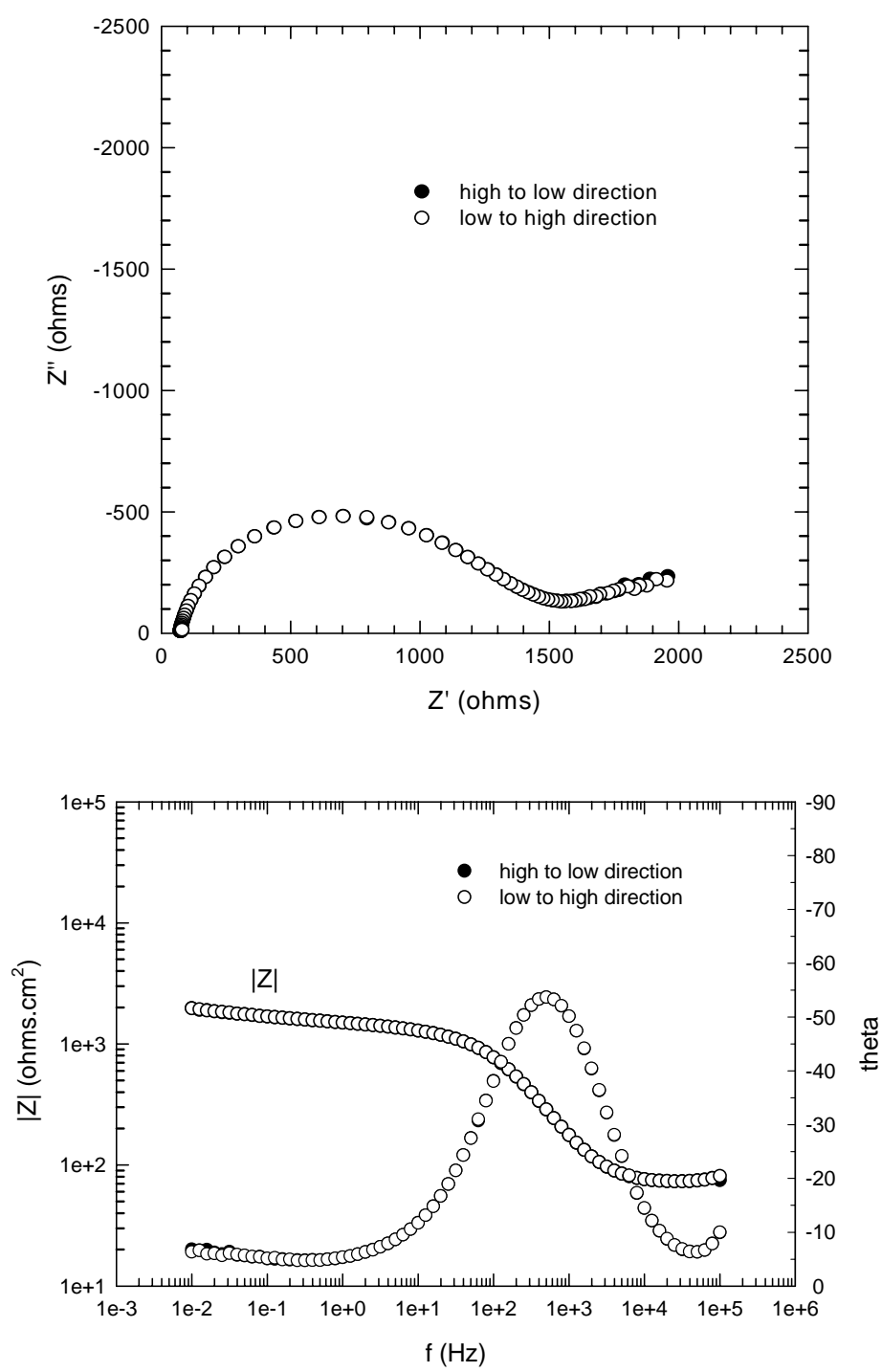

Figure 6-11: Comparison of Nyquist (a) and Bode (b) plots for passive zirconium formed at $0.1 \mathrm{~V}_{\text {she }}$ for 24 hours by scanning the frequency in the high-to-low direction and immediately from the low-to-high direction in 0.1 $\mathrm{M} \mathrm{B}(\mathrm{OH})_{3}+0.001 \mathrm{M} \mathrm{LiOH}$ solution with $22 \mathrm{~cm}^{3}(\mathrm{STP}) / \mathrm{kg}\left(\mathrm{H}_{2} \mathrm{O}\right)$ hydrogen. T $=250{ }^{\circ} \mathrm{C}$ and $\mathrm{P}=62$ bar. "High-to-low" means stepping the frequency from 
high to low direction and "low-to-high" means stepping the frequency in the reverse direction.

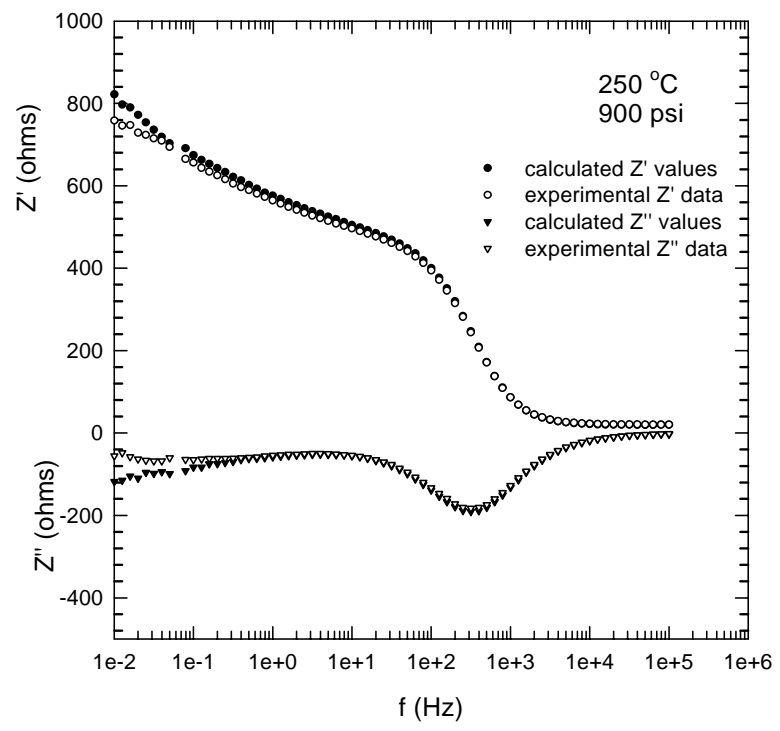

Figure 6-12: Typical Kramers-Kronig transforms of the real and imaginary components of the impedance of passive zirconium in $0.1 \mathrm{M} \mathrm{B}(\mathrm{OH})_{3}+0.001 \mathrm{M}$ $\mathrm{LiOH}$ solution at $\mathrm{T}=250^{\circ} \mathrm{C}$ and $\mathrm{P}=62$ bar. The oxide film was formed at $0.6 \mathrm{~V}_{\text {she }}$. "calculated" real and imaginary components were derived from the experimental imaginary and real components, respectively, by using the $\mathrm{K}-\mathrm{K}$ transforms. These data are compared with the experimental real and imaginary components of the impedance.

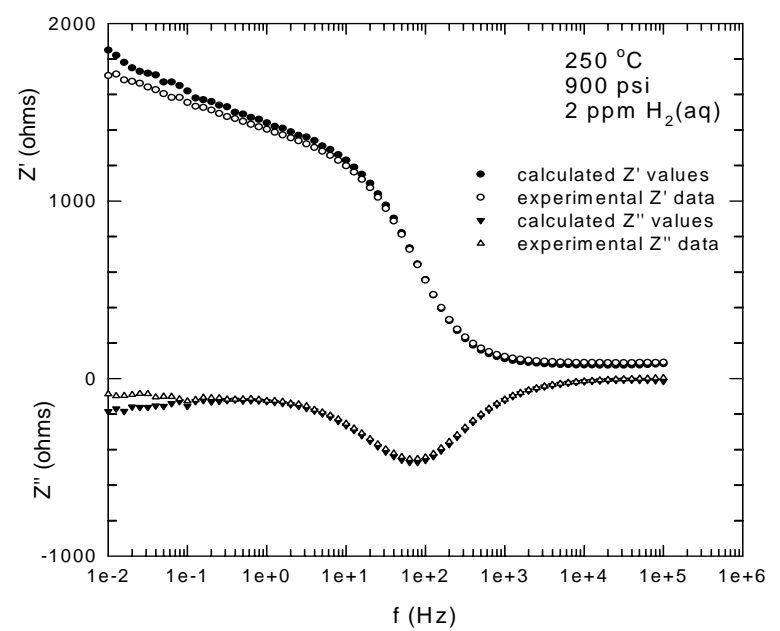

Figure 6-13. Typical Kramers-Kronig transforms of the real and imaginary components of the impedance of passive zirconium in $0.1 \mathrm{M} \mathrm{B}(\mathrm{OH})_{3}+0.001 \mathrm{M}$ $\mathrm{LiOH}$ solution with $22 \mathrm{~cm}^{3}(\mathrm{STP}) / \mathrm{kg}\left(\mathrm{H}_{2} \mathrm{O}\right)$ hydrogen (aq) at $\mathrm{T}=250^{\circ} \mathrm{C}$ and $\mathrm{P}=62$ bar. The oxide film was formed at a formation potential $0 \mathrm{~V}_{\text {she }}$ "calculated" real and imaginary components were derived from the experimental imaginary and real 
components, respectively, by using the K-K transforms. These data are compared with the experimental real and imaginary components of the impedance.
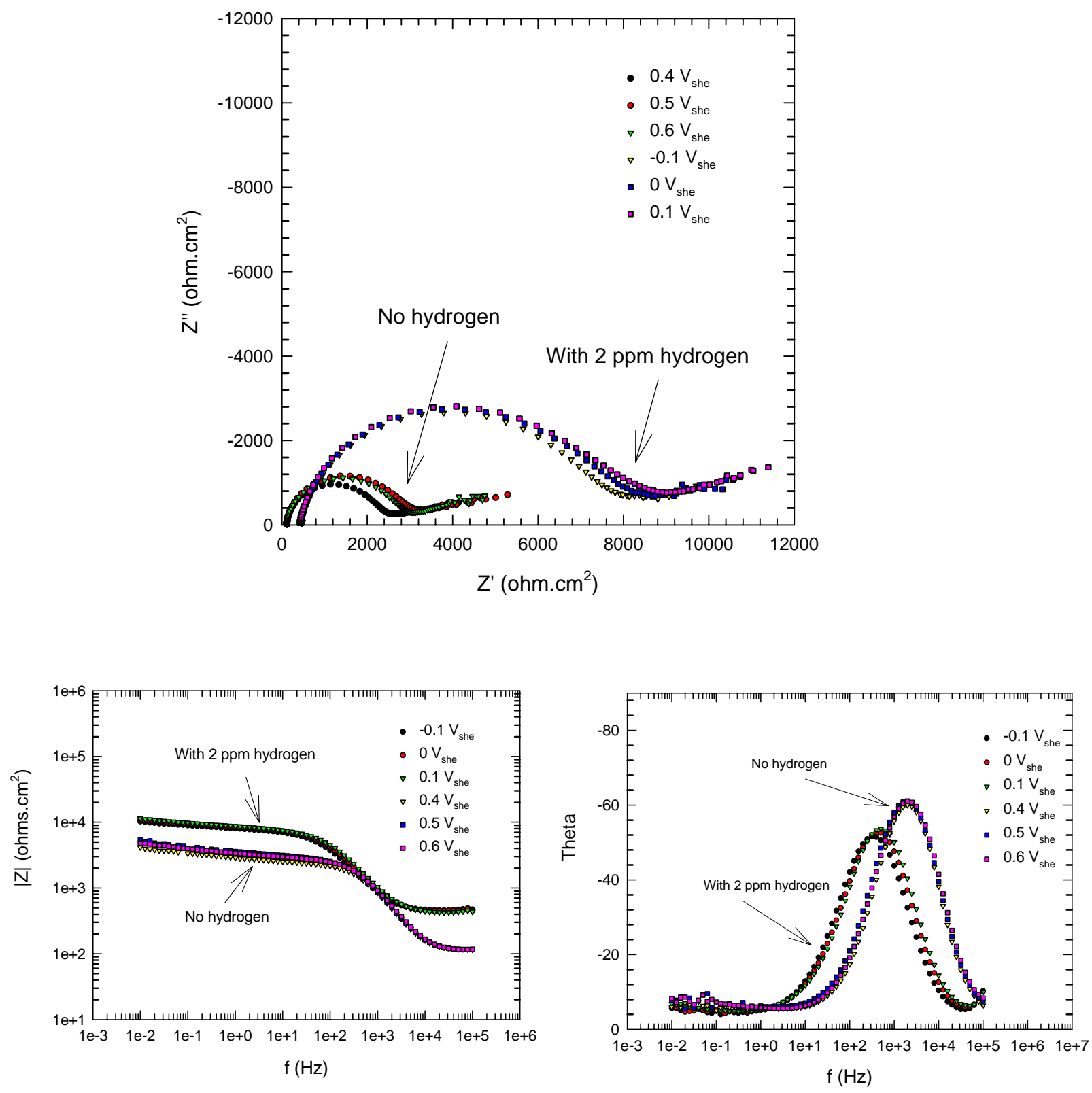

Figure 6-14: Impedance spectra for passive zirconium as a function of film formation potential in $0.1 \mathrm{M} \mathrm{B}(\mathrm{OH})_{3}+0.001 \mathrm{M} \mathrm{LiOH}$ solutions with and without added hydrogen. The impedance was measured after holding the potential at each step for 24 hours for film stabilization. The potentials was changed in the negative-to-positive direction. $\mathrm{T}=250{ }^{\circ} \mathrm{C}$ and $\mathrm{P}=62 \mathrm{bar}$. 
The quality of the impedance data were also checked using the Kramers-Kronig transforms. These integral transforms test for compliance of the system with the linearity, stability, and causality constraints of Linear Systems Theory (LST). The K-K transforms, which arise from Cauchy's theorem and the definition of causality were first developed for the field of optics by establishing a connection between the real and imaginary parts of complex optical parameters for the system. The K-K transforms are a purely mathematical results and do not reflect any physical property of the tested system. Thus, only the experimental data satisfying the linearity, stability, and causality constraints, as indicated by the K-K transforms, can be used to describe the properties of the systems in terms of linear response theory. Macdonald and Urquidi-Macdonald [16-18] derived the mathematical relations transforming the real part of electrochemical impedance into the imaginary part of the impedance and vice versa. Their studies show that the K-K transform are strongly sensitive to the violation of the stability constraint but are not particularly sensitive to violation of the linearity condition. Figure 6-12 and Figure 6-13 show two sets of K-K transforms in which the transformed components of the impedance are compared with the corresponding measured components for systems with hydrogen and without hydrogen. Excellent agreement is obtained, except for the small descepancies at low frequencies; the discrepancies are due to the "tails" problem that arises from the fact that the impedance data are measured over a finite bandwidth whereas the transforms are defined over an infinite bandwidth in frequency.

Figures 6-14 shows Nyquist and Bode plots for passive film formed on zirconium surface measured at various potentials in the passive range in $0.1 \mathrm{M} \mathrm{B}(\mathrm{OH})_{3}+0.001 \mathrm{M}$ $\mathrm{LiOH}$ solution with argon or with hydrogen gas at $250^{\circ} \mathrm{C}$ and 62 bar. The magnitude of the impedance of the passive film formed in the presence of hydrogen is much higher than that formed without hydrogen, in good correlation with the passive current behavior and the film thickness data discussed above. In terms of Point Defect Model, the higher impedance of the interface exposed to hydrogenated solutions can be attributed to a thicker outer layer, which probably results from a higher rate of formation than in the BWR case. Thus, for PWR environments, the thermodynamic data are consistent with the barrier layer being a defective hydride, possibly $\mathrm{ZrH}_{2-\mathrm{x}}$. It is likely that the defect is exclusively the hydrogen vacancy, so that formation of the outer layer will occur by hydrolysis of the hydride, rather than via restructuring of the outer surface of the barrier layer or the hydrolysis/precipitation of cations transmitted through the barrier layer, as in the simulated BWR case. This reaction

$$
\mathrm{ZrH}_{2}(\text { barrier layer })+2 \mathrm{H}_{2} \mathrm{O} \rightarrow \mathrm{ZrO}_{2}(\text { porous outer layer })+3 \mathrm{H}_{2}
$$

is likely to be fast compared with the corresponding reaction for the BWR case

or

$$
\left.\begin{array}{l}
\mathrm{ZrO}_{2}(\text { barrier layer }) \rightarrow \mathrm{ZrO}_{2}(\text { porous outer layer }) \\
\mathrm{Zr}^{4+}+2 \mathrm{H}_{2} \mathrm{O} \rightarrow \mathrm{ZrO}_{2}(\text { porous outer layer })+4 \mathrm{H}^{+}
\end{array}\right\}
$$


Since the rates of dissolution of the outer layers in both cases are likely to be similar, the higher rate of Reaction (6-12) compared with Reaction (6-13) should lead to a thicker, more resistive film in the PWR case than in the BWR case, as observed.

The shapes of the Nyquist plots for zirconium in the solutions with and without hydrogen are virtually the same, both showing depressed semicircles at high frequencies and a Warburg tail of constant phase angle of $4-8^{\circ}$ according to Fig. 11 at low frequencies $(\mathrm{f}<1 \mathrm{~Hz})$. The impedance is insensitive to film formation potential within the passive range. In terms of the postulates of the PDM, the electric field strength is independent of the formation potential, and the transport of the defects is driven by the electric field strength. Therefore, the impedance should be insensitive to the film formation potential as has been observed for other systems [19]. However, as argued above, the impedance is dominated by the properties of the outer layer and it is unlikely that the barrier layer makes a significant contribution other than being the sources of outer layer material, as described by Reactions (6-12) and (6-13).

Time evolution of passive film impedance was checked and the impedance didn't change much for 1 hour and 12 hours immersion time. The impedance was almost independent of the immersion time for $t>12$ hours (Figure 6-15) which means the growth of protective passive film very fast.

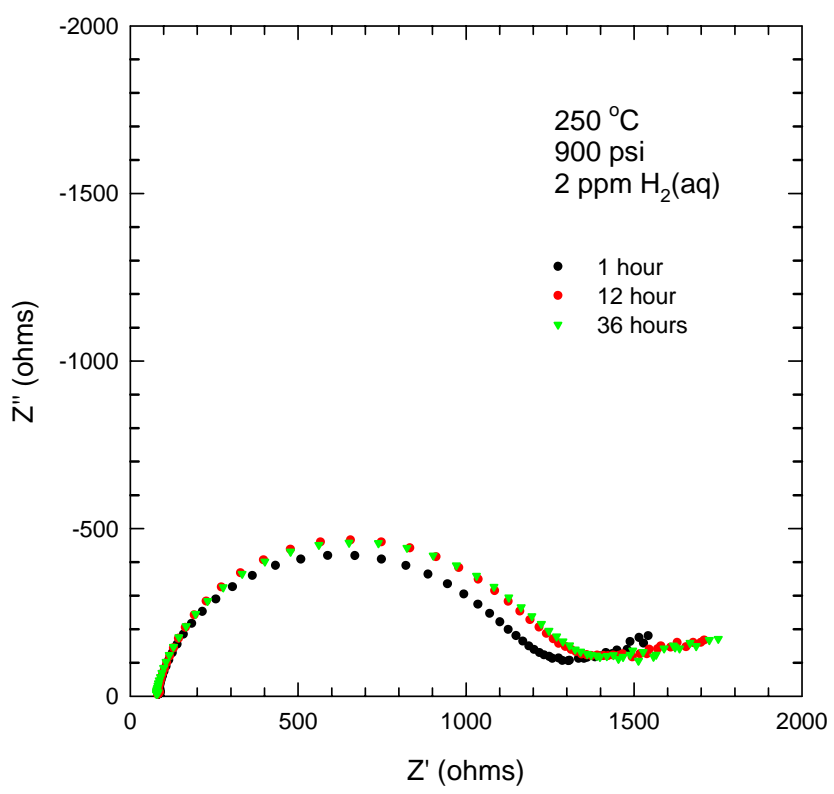

Figure 6-15: Time evolution of oxide film impedance of zirconium formed at potential $-0.1 \mathrm{~V}_{\text {she }}$ in $0.1 \mathrm{M} \mathrm{B}(\mathrm{OH})_{3}+0.001 \mathrm{M} \mathrm{LiOH}$ solution with 22 $\mathrm{cm}^{3}(\mathrm{STP}) / \mathrm{kg}\left(\mathrm{H}_{2} \mathrm{O}\right)$ hydrogen. $\mathrm{T}=250{ }^{\circ} \mathrm{C}$ and $\mathrm{P}=62$ bar. 


\section{Mott-Schottky Analysis}

An understanding of the crystallographic and electronic defect structures of the passive film on zirconium is vital in exploring the underlying mechanisms of oxidation and hydriding of Zircaloy fuel cladding in reactor coolant environments. To identify the principal defect in the passive film, Mott-Schottky (M-S) analysis [2] is being used. In M-S analysis, the capacitance of the passive film is measured at a suitably high frequency as a function of voltage. According to Mott-Schottky theory [20], the space charge capacitance $C_{s c}(\mathrm{~F})$ of n-type and p-type semiconductor junctions are given by

and

$$
\frac{1}{C_{s c}^{2}}=\frac{2}{\varepsilon \varepsilon_{0} q N_{D} A^{2}}\left(E-E_{f b}-\frac{k T}{q}\right) \quad \text { n-type }
$$

$$
\frac{1}{C_{s c}^{2}}=\frac{-2}{\varepsilon \varepsilon_{0} q N_{A} A^{2}}\left(E-E_{f b}-\frac{k T}{q}\right) \quad \text { p-type }
$$

respectively. In these expressions, $A$ is the surface area of the electrode $\left(\mathrm{cm}^{2}\right), N_{D} / N_{A}$ is the donor/acceptor concentration $\left(\mathrm{cm}^{-3}\right), \varepsilon_{0}$ is the vacuum permittivity $\left(8.85 \times 10^{-14} \mathrm{~F} / \mathrm{cm}\right)$, $\varepsilon$ is the dielectric constant of the oxide, $E$ is the applied potential, $E_{f b}$ is the flat band potential, $q$ is the electron charge $\left(1.6 \times 10^{-19} \mathrm{C}\right)$, and $k T / q$ is about $45 \mathrm{mV}$ for $250{ }^{\circ} \mathrm{C}$. In addition to confirming the electronic nature of the film, M-S analysis yields the dopant concentration (donor or acceptor) in the film as a function of voltage. The validity of this approach is based on the assumption that the capacitance of the space charge layer $C_{s c}$ is measured as the electrode capacity. It is assumed that any other series capacitance associated with the electrode/electrolyte interface (e.g. the double layer capacitance) is much larger than the space charge capacitance. Accordingly, a $C_{p}^{-2}$ versus $\mathrm{V}$ profile should be a straight line with a slope that is inversely proportional to the doping concentration.

The outer layer of the passive film formed on zirconium is found to be much thicker than the barrier layer. The thin barrier layer is a defective phase with semiconductor properties that depend upon the type of defects present while the outer layer is relatively porous and has no appreciable semiconductor properties [6]. Therefore, the measured capacitance cannot be directly approximated as the space charge capacitance; instead, the observed capacitance $C$ can be understood by postulating that it reflects a series combination of a voltage-independent capacitance, $C_{o x}$, due to the outer layer insulting layer, and the space charge capacitance, $C_{b l}$, of the barrier layer, as discussed in the thickness calculation section of this paper.

$$
\frac{1}{C}=\frac{1}{C_{o x}}+\frac{1}{C_{b l}}
$$


It has been shown by de Gryse et al. [21] that the measured capacitance can be related to $C_{o x}$ and the applied voltage by

$$
\frac{1}{C^{2}}=\frac{1}{C_{o x}^{2}}+\frac{2}{\varepsilon \varepsilon_{0} q N_{D}}\left(V-V_{f b}-\frac{k T}{q}\right)
$$

where the notation is same as previously defined. As can be seen from this expression, the slope of the MS-plot is not influenced by the oxide capacitance $C_{o x}$ and can be used to derive reliable values for the donor density, $N_{D}$, from the slope of the measured MS-plot, although the intercept is shifted on the vertical and horizontal axes, thereby yielding a flat band potential that is too negative. Mott-Schottky analysis can be applied to a system of fixed (voltage-independent) total dopant concentration and, hence, $C$ versus $V$ must be measured under the conditions that do not result in a change in the dopant concentration profile of the film. In this work, after holding the specimen at the desired potential for 24 hours, in order for the film to reach steady state, the capacitance was recorded while simultaneously sweeping the voltage in the negative direction from the formation potential at a sweep rate of $25 \mathrm{mV} / \mathrm{s}$. This sweep rate is considered to be sufficiently high that the defect structure is "frozen-in" and hence that $N_{D}$ in Eq. (6-17) appears as a constant (identical M-S slopes were obtained for different scan rates over $25 \mathrm{mV} / \mathrm{s}$, as shown in Fig. 6-16). Mott-Schottky plots are presented in Fig. 6-17 and Fig. 6-18 for passive $\mathrm{Zr}$ formed in hydrogenated and hydrogen-free solutions at different formation potentials. From the positive slopes, it is concluded that the passive films on zirconium formed in high temperature borate buffer solutions with or without hydrogen exhibits ntype electronic character. This result shows that the dominant defects in the film over this voltage range are oxygen/hydrogen vacancies and/or zirconium interstitials.

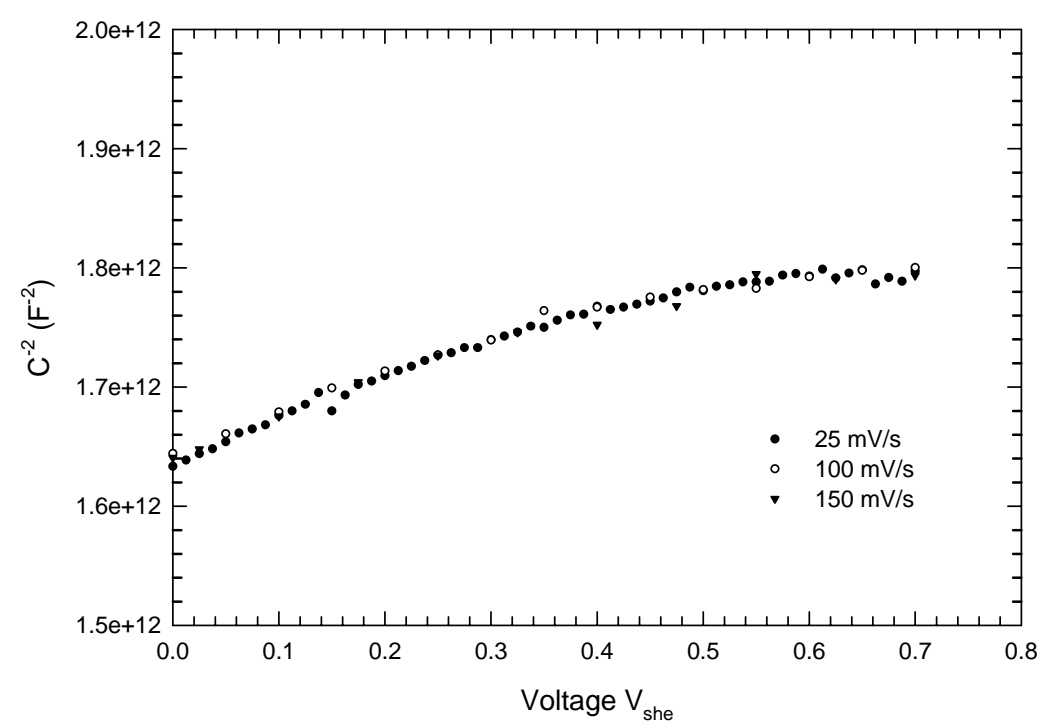

Figure 6-16: Mott-Schottky plots for the $\mathrm{Zr}$ passive film formed at $0.6 \mathrm{~V}_{\text {she }}$ potential in $0.1 \mathrm{M} \mathrm{B}(\mathrm{OH})_{3}+0.001 \mathrm{M} \mathrm{LiOH}$ solution without hydrogen in the reservoir, measured at different scan rates. Sinusoidal excitation frequency $=$ 
$15 \mathrm{kHz} . \mathrm{T}=250^{\circ} \mathrm{C}$ and $\mathrm{P}=900$ psi. No dependency was found for scan rate $\geq$ $25 \mathrm{mV} / \mathrm{s}$.

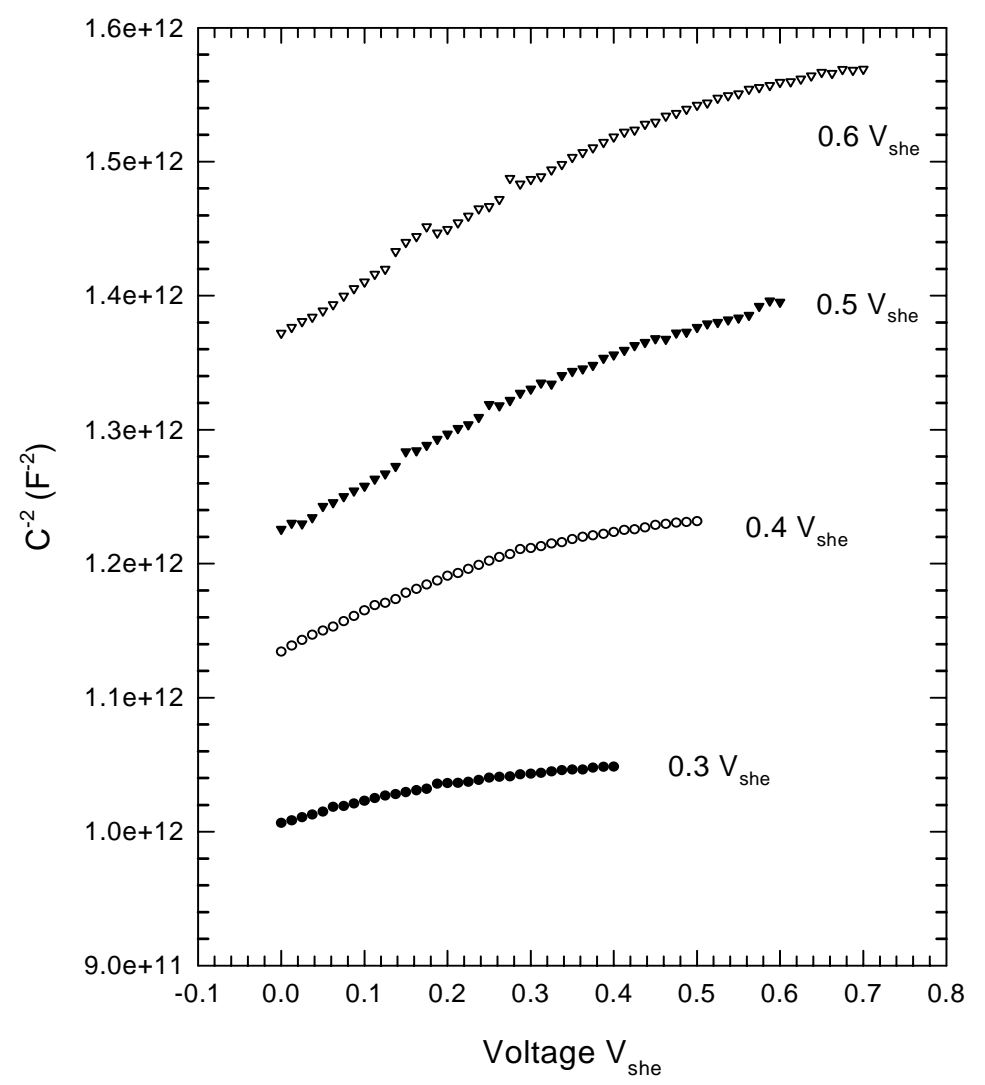

Figure 6-17: Mott-Schottky plots for the $\mathrm{Zr}$ passive film formed at the indicated potentials after stabilization for 24 hours in $0.1 \mathrm{M} \mathrm{B}(\mathrm{OH})_{3}+0.001$ $\mathrm{M}$ LiOH solution sparged with argon gas. Voltage sweep rate in the negative direction from the formation voltage $=25 \mathrm{mV} / \mathrm{s} . \mathrm{T}=250{ }^{\circ} \mathrm{C}$ and $\mathrm{P}=62$ bar. 


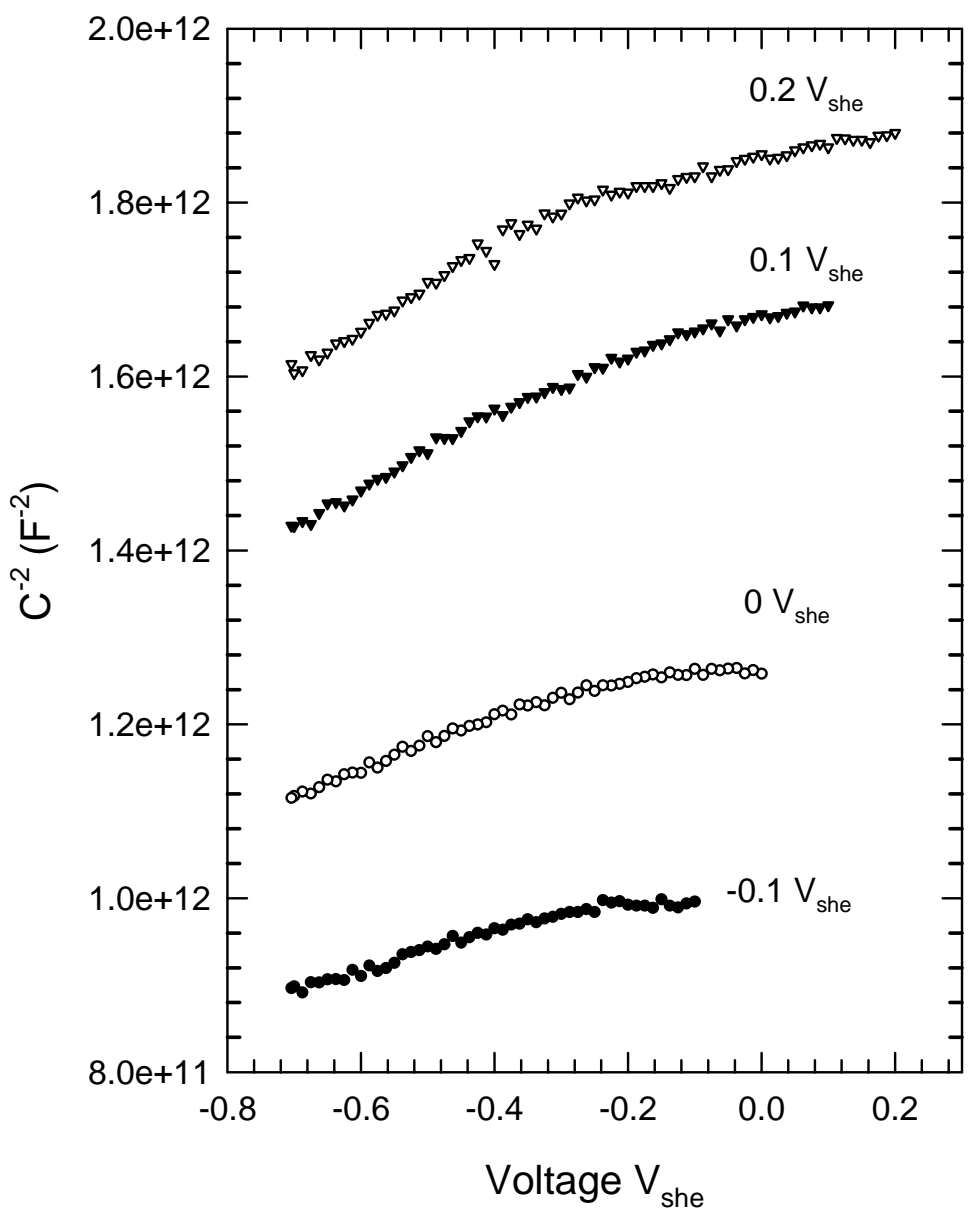

Figure 6-18: Mott-Schottky plots for passive $\mathrm{Zr}$ at the indicated potentials after stabilization for 24 hours in $0.1 \mathrm{M} \mathrm{B}(\mathrm{OH})_{3}+0.001 \mathrm{M} \mathrm{LiOH}$ solution with $22 \mathrm{~cm}^{3}(\mathrm{STP}) / \mathrm{kg}\left(\mathrm{H}_{2} \mathrm{O}\right)$ hydrogen. Voltage sweep rate in the negative direction from the formation voltage $=25 \mathrm{mV} / \mathrm{s}$. $\mathrm{T}=250{ }^{\circ} \mathrm{C}$ and $\mathrm{P}=62 \mathrm{bar}$.

The donor density $N_{D}$ versus the formation potential (Fig. 6-19) has been calculated from the slopes in Fig. 6-17 and Fig. 6-18 for passive films formed in the hydrogenated and hydrogen-free solutions. The data show that the donor concentration in the vicinity of the metal/film interface decreases with increasing formation potential for both cases, in agreement with the prediction of the Point Defect Model. The donor concentration, $N_{D}$, is in the range of $10^{17} \sim 10^{18} \mathrm{~cm}^{-3}$, which agrees with the $N_{D}<10^{19} \mathrm{~cm}^{-3}$ reported by Meisterjahn [22] for the zirconium passive film formed at ambient temperature. The much lower donor concentration found here demonstrates that the film is only lowly-doped with electron donors. However, the low doping level probably reflects a composite value for a highly doped, but thin, barrier layer and a thick stoichiometric outer layer. 


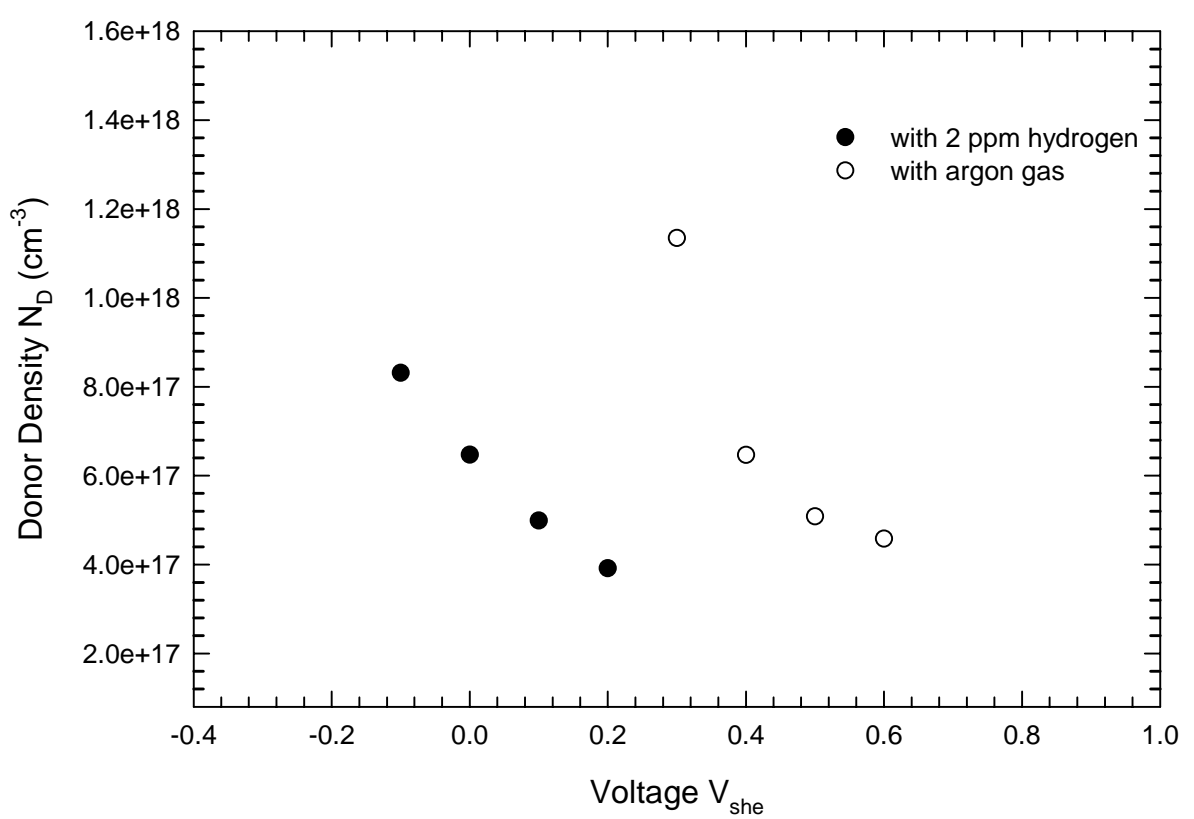

Figure 6-19: Donor density $N_{D}$ of the passive film formed on $\mathrm{Zr}$ as a function of film formation potential in $0.1 \mathrm{M} \mathrm{B}(\mathrm{OH})_{3}+0.001 \mathrm{M} \mathrm{LiOH}$ solutions with $22 \mathrm{~cm}^{3}(\mathrm{STP}) / \mathrm{kg}\left(\mathrm{H}_{2} \mathrm{O}\right)$ or without hydrogen. $\mathrm{T}=250{ }^{\circ} \mathrm{C}$ and $\mathrm{P}=62$ bar.

In this part of work, the electrochemistry of zirconium has been explored in situ in $0.1 \mathrm{M} \mathrm{B}(\mathrm{OH})_{3}+0.001 \mathrm{M} \mathrm{LiOH}$ aqueous solution $\left(\mathrm{pH}=6.94\right.$ at $\left.250{ }^{\circ} \mathrm{C}\right)$ with and without added hydrogen to simulate the water chemistries of the coolant circuits in Pressurized Water Reactors (PWRs) and Boiling Water Reactors (BWRs), respectively. Potentiostatic polarization, potentiodynamic polarization, and electrochemical impedance spectroscopy (EIS) were used, with the latter also being employed for determining the passive film thickness and for carrying out Mott-Schottky analyses. The principal findings and conclusions of this part of work are as follows:

1) From thermodynamic analysis, it is postulated that a hydride barrier layer forms under PWR coolant conditions, whereas an oxide barrier layer forms under BWR primary coolant conditions. Thus, the introduction of hydrogen into the solution lowers the corrosion potential of zirconium to the extent that the formation of $\mathrm{ZrH}_{2}$ is predicted to be spontaneous rather than the $\mathrm{ZrO}_{2}$.

2) The logarithm of the steady state passive current density is found to be independent of formation potential, while the steady-state film thickness $\left(L_{S S}\right)$ is a linear function of the formation potential. The much greater anodizing constants $\left(\partial L_{s s} / \partial V\right)_{\mathrm{pH}}$ than for other metals and alloys at ambient temperature suggest that a thick outer layer forms over a thin barrier layer. 
3) The Nyquist plots of the impedance are in the form of depressed semicircles with a Warburg like impedance at low frequencies for both the PWR and BWR cases. The magnitude of impedance of the passive film formed in the hydrogenated solution is much higher than that for the film formed in the hydrogen free solution, which is consistent with the measured passive currents for the two cases.

4) Mott-Schottky analysis shows that the passive film formed anodically on zirconium under hydrogenated and dehydrogenated environments is n-type in electronic character, corresponding to a preponderance of oxygen/hydrogen vacancies and/or zirconium interstitials, with the former being likely, in the barrier layer.

5) The n-type electronic character of the film is consistent with the diagnostic criteria offered by Point Defect Model.

6) The donor concentration is in the range of $10^{17} \sim 10^{18} \mathrm{~cm}^{-3}$, which decreases with increasing formation potential, demonstrating that the film is only lowly-doped with electron donors. However, the low doping level probably reflects a composite value for a highly doped, but thin, barrier layer and a thick, stoichiometric outer layer.

\subsection{Transient Analysis of Zirconium}

According to the Point Defect Model (PDM), the rate of change in thickness of the barrier layer of the passive film can be written as

$$
\frac{d L}{d t}=a \cdot e^{-b L}-c
$$

where $a=\Omega k_{3}^{0} e^{\alpha_{3}(1-\alpha) \chi \gamma V} e^{-c_{3} \chi \beta p H}, b=\alpha_{3} \chi \varepsilon \gamma, c=\Omega k_{7}\left(C_{H^{+}} / C_{H^{+}}^{0}\right)^{n}, \varepsilon$ is the electric field strength, $\mathrm{C}_{\mathrm{H}}{ }^{+}$is the concentration of hydrogen ion at the film/solution interface, $C_{H^{+}}^{0}$ is the standard state concentration, such that $k_{7}$ (the rate constant for the dissolution of the film) has units of $\mathrm{mol} / \mathrm{cm}^{2} \mathrm{~s}, \Omega$ is the mole volume of the film per cation, $n$ is the kinetic order of the dissolution reaction at the film/solution interface with respect to $\mathrm{H}^{+}, \alpha$ is the polarizability of the film/solution interface, $\beta$ is the dependence of the potential drop across the film/solution interface on the $\mathrm{pH}, V$ is the applied voltage, $\gamma=F / R T, F$ is Faraday's constant, $R$ is the universal gas constant, $T$ is the Kelvin temperature, and $\alpha_{3}$ and $k_{3}^{0}\left(\mathrm{~mol} / \mathrm{cm}^{2} \mathrm{~s}\right)$ are the transfer coefficient and the standard rate constant, respectively, for the generation of oxygen vacancies at the metal/film interface.

Eq. (6-18) is readily integrated over the limits $\left(L_{0}, t=0, V\right)$ to $(L, t, V+\Delta \mathrm{V})$, where $\Delta \mathrm{V}$ is the step in voltage at $t=0$, to yield the transients in anodic oxide film thickness and current density given by Equations (6-19) and (6-20).

$$
L(t)=L_{0}+\left(\frac{1}{b}\right) \ln \left[\left(\frac{a^{\prime}}{c}\right) e^{-b L_{0}}\left(e^{b c t}-1\right)+1\right]-c t
$$




$$
i=\chi F k_{3}^{0} e^{a_{3} V} e^{-c_{3} p H} X+(\delta-\chi) F k_{7}^{0} e^{a_{7} V} e^{c_{7} p H}\left(C_{H^{+}} / C_{H^{+}}^{0}\right)^{n}
$$

respectively, where

$$
X=\frac{c e^{-b_{3} L_{0}}}{a^{\prime} e^{-b_{3} L_{0}}-\left(a^{\prime} e^{-b_{3} L_{0}}-c\right) e^{-b c t}}
$$

With a,b, and $\mathrm{c}$ being as defined in equation 6-18. Note that $c$ is the dissolution rate at voltage $V+\Delta \mathrm{V}$, and that $a^{\prime}=k_{3}^{0} e^{\alpha_{3}(1-\alpha) \gamma(V+\Delta V)} e^{-\alpha_{3} \beta p H}$. Note also that Equations (6-19) and (6-20) apply strictly to an oxygen ion conducting film, although it is believed (but not proven) that the theory is also applicable to cation interstitial conducting barrier layers as well.

As we can see, if $\Delta \mathrm{V}$ is sufficiently negative that the first term in Eq. 6-18 is negligible with respect to the second term, and hence that $\frac{d L}{d t} \approx-c$ (a constant for a given $\mathrm{pH})$. So $c$ can be easily estimated from the slope of capacitance transient. Eventually, the two terms on the right side of Eq. 6-18 become equal, principally due to the increase in the potential drop across the metal/film interface, as the film thins. Film thinning, due to dissolution, give rise to a continual increase in the value of the first term in Eq. 6-18, eventually resulting in the attainment of a steady state. So that

$$
L_{s s}=(1 / b) \ln (a / c) .
$$

Upon substitution of the constants defined above, it is straightforward to show that this equation is identical to the expression that was previously derived for the steady state thickness of the film (barrier layer), namely,

$$
L_{s s}=\frac{1}{\varepsilon}\left[1-\alpha-\frac{\alpha \alpha_{7}}{\alpha_{3}}\left(\frac{\delta}{\chi}-1\right)\right] V+\frac{1}{\varepsilon}\left\{\frac{2.303 n}{\alpha_{3} \chi \gamma}-\beta\left[\frac{\alpha_{7}}{\alpha_{3}}\left(\frac{\delta}{\chi}-1\right)+1\right]\right\} p H+\frac{1}{\alpha_{3} \chi K} \ln \left(\frac{k_{3}^{0}}{k_{7}^{0}}\right)
$$

where $K=\varepsilon \gamma$, and others are same as above defined. If we assume no change in oxidation state occurs, a simpler expression results in the form:

$$
L_{s s}=\left(\frac{1-\alpha}{\varepsilon}\right) V+\frac{1}{\varepsilon}\left\{\frac{2.303 n}{\alpha_{3} \chi \gamma}-\beta\right\} p H+\frac{1}{\alpha_{3} \chi K} \ln \left(\frac{k_{3}^{0}}{k_{7}^{0}}\right)
$$

As noted earlier, that the barrier layer on zirconium under PWR fuel cladding conditions is the defective hydride $\left(\mathrm{ZrH}_{2-\mathrm{x}}\right)$ and that the outer layer is the porous oxide $\left(\mathrm{ZrO}_{2}\right)$, whereas in the BWR case the barrier layer is the defective oxide $\left(\mathrm{ZrO}_{2-\mathrm{x}}\right)$ and the outer layer is the porous, non-defective $\mathrm{ZrO}_{2}$. Only the barrier layer, which is a small (but important) part of the passive film, has been postulated as being a hydride. The barrier layer is typically a few tens of nanometers thick, whereas the oxide outer layer may be many microns thick depending upon the conditions, so that the great bulk of the 
passive film is still the oxide, even under highly reducing PWR primary coolant conditions. We adopt these models as working hypotheses in the present work, with the formation of the defective oxide (BWR) and hydride (PWR) barrier layers being described in the classic Point Defect Model format by Figs 6-20(a) and 6-20 (b) respectively.

\section{\begin{tabular}{l|l|l} 
Metal & Barrier Oxide Layer $\mathrm{ZrO}_{2-\mathrm{x}}$ & Porous $\mathrm{ZrO}_{2}$ Outer Layer/Solution
\end{tabular}}
(1) $Z r+V_{Z r}^{4^{\prime}} \stackrel{k_{1}}{\longrightarrow} Z r_{Z r}+v_{Z r}+4 e^{\prime}$
(4) $\mathrm{Zr}_{\mathrm{Zr}}+2 \mathrm{OH}^{-} \stackrel{k_{4}}{\longrightarrow} \mathrm{ZrO}^{2+}+\mathrm{V}_{\mathrm{Zr}}^{4}+\mathrm{H}_{2} \mathrm{O}$
(2) $Z r \stackrel{k_{2}}{\longrightarrow} Z r_{i}^{4+}+v_{Z r}+4 e^{\prime}$
(5) $\mathrm{Zr}_{i}^{4+}+2 \mathrm{OH}^{-} \stackrel{k_{5}}{\longrightarrow} \mathrm{ZrO}^{2+}+\mathrm{H}_{2} \mathrm{O}$
(3) $Z r \stackrel{k_{3}}{\longrightarrow} Z r_{Z r}+2 V_{O}{ }^{\prime}+4 e^{\prime}$
(6) $\mathrm{V}_{O}^{*}+\mathrm{H}_{2} \mathrm{O} \stackrel{k_{6}}{\longrightarrow} \mathrm{O}_{O}+2 \mathrm{H}^{+}$
(7) $\mathrm{ZrO}_{2}+2 \mathrm{H}^{+} \stackrel{k_{7}}{\longrightarrow} \mathrm{ZrO}^{2+}+\mathrm{H}_{2} \mathrm{O}$

Figure 6-20 (a). Schematic of the reactions that are postulated to occur at the metal/barrier layer and barrier layer/outer layer interfaces during the formation of the passive film on zirconium under BWR-like conditions according to the Point Defect Model. Note that Reactions (1), (2), (4), (5), and (6) are lattice conservative processes, in that their occurrence does not result in the movement of the interface, whereas Reactions (3) and (7) are nonconservative. $Z r=$ Zirconium atom, $V_{Z r}^{4^{4}}=$ cation vacancy on the zirconium sub lattice of the barrier layer, $v_{z r}=$ zirconium vacancy in the metal, $Z r_{i}^{4+}=$ cation interstitial in the film, $Z r_{Z r}=$ zirconium cation on the metal sub lattice of the barrier layer, $V_{o}=$ oxygen vacancy on the oxygen sub lattice of the barrier layer, $O_{O}=$ oxygen anion on the oxygen sub lattice of the barrier layer.

\section{Metal | Barrier Hydride Layer $\mathrm{ZrH}_{2-}$ ～Porous $\mathrm{ZrO}_{2}$ Outer Layer/Solution}
(1) $Z r+V_{Z r}^{2 \prime} \stackrel{k_{1}}{\longrightarrow} Z r_{Z r}+v_{Z r}+2 e^{\prime}$
(4) $\mathrm{Zr}_{\mathrm{Zr}}+2 \mathrm{OH}^{-} \stackrel{k_{4}}{\longrightarrow} \mathrm{ZrO}^{2+}+\mathrm{V}_{\mathrm{Zr}}^{2 \prime \prime}+\mathrm{H}_{2} \mathrm{O}+2 e^{\prime}$
(2) $Z r \stackrel{k_{2}}{\longrightarrow} Z r_{i}^{2+}+v_{Z r}+2 e^{\prime}$
(5) $\mathrm{Zr}_{i}^{2+}+2 \mathrm{OH}^{-} \stackrel{k_{5}}{\longrightarrow} \mathrm{ZrO}^{2+}+\mathrm{H}_{2} \mathrm{O}+2 e^{\prime}$
(3) $Z r \stackrel{k_{3}}{\longrightarrow} Z r_{Z r}+2 V_{H}+2 e^{\prime}$
(6) $2 \mathrm{~V}_{\mathrm{H}}+2 \mathrm{H}_{2} \mathrm{O}+4 e^{-} \stackrel{k_{6}}{\longrightarrow} 2 \mathrm{H}_{\mathrm{H}}+2 \mathrm{OH}^{-}$
(7) $\mathrm{ZrH}_{2}+4 \mathrm{OH}^{-} \stackrel{k_{7}}{\longrightarrow} \mathrm{ZrO}^{2+}+3 \mathrm{H}_{2} \mathrm{O}+6 e^{\prime}$
(8) $\mathrm{ZrH}_{2}+2 \mathrm{H}_{2} \mathrm{O} \stackrel{k_{8}}{\longrightarrow} \mathrm{ZrO}_{2}+3 \mathrm{H}_{2}$

Figure 6-20 (b). Schematic of the reactions that are postulated to occur at the metal/barrier layer and barrier layer/outer layer interfaces during the formation of the passive film on zirconium under PWR-like conditions according to the Point Defect Model. Note that Reactions (1), (2), (4), (5), and (6) are lattice conservative processes, in that their occurrence does not result in the movement of the interface, whereas Reactions (3), (7), and (8) are nonconservative. $V_{z r}^{2 \prime}=$ cation vacancy on the zirconium sub lattice of the barrier layer, $\mathrm{Zr}_{i}^{2+}=$ cation interstitial in the film, $V_{H}=$ hydrogen vacancy on the hydrogen sub lattice of the barrier layer, $H_{H}=$ hydrogen anion on the hydrogen sub lattice of the barrier layer. Note that the destruction of the 
hydride barrier layer is postulated to occur electrochemically [Reaction (7)] and chemically [Reaction (8)].

In Fig. 6-20 (b), two decomposition processes are postulated for $\mathrm{ZrH}_{2}$; an electrochemical process resulting in the formation of the zirconyl cation $\left[\mathrm{ZrO}^{2+}\right.$, Reaction (7)] and a chemical process [Reaction (8)] that forms the oxide, $\mathrm{ZrO}_{2}$, and hydrogen. Note that in the oxidation of $\mathrm{ZrH}_{2}$ to form $\mathrm{ZrO}^{2+}$ the oxidation state of zirconium changes from +2 to +4 and that the oxidation state of hydrogen changes from -1 (in $\mathrm{ZrH}_{2}$ ) to +1 in $\mathrm{H}_{2} \mathrm{O}$. Accordingly, the total change in oxidation state in transforming $\mathrm{ZrH}_{2}$ into $\mathrm{ZrO}^{2+}$ is +6 compared with zero for the transformation of $\mathrm{ZrO}_{2}$ to $\mathrm{ZrO}^{2+}$ in the postulated BWR case [Reaction (7), Fig. 6-20(a)].

A Solartron Model 1287 Electrochemical Interface was used to control the potential for film growth or thinning over the potential cycle. The capacitance was monitored with a Solartron 1255B Frequency Response Analyzer using an excitation voltage of $10 \mathrm{mV}$ (peak-to-peak) and an applied frequency of $1 \mathrm{kHz}$. The current and capacitance changes over the potential stepping cycle were recorded simultaneously. The film thickness was estimated from capacitance measurements, where at frequencies higher than $1 \mathrm{kHz}$, the electrochemical impedance displays an almost purely capacitive frequency response. Accordingly, we use the well known parallel plate expression for the capacitance, $\mathrm{C}$, to estimate the thickness of the film, L, as

$$
L=\frac{\varepsilon \varepsilon_{0} A}{C}
$$

where $A$ is the surface area, $\varepsilon$ is the dielectric constant of the oxide film $(\sim 22$ for zirconium oxide), and $\varepsilon_{0}$ is the vacuum permittivity $\left(8.85^{*} 10^{-14} \mathrm{~F} / \mathrm{cm}\right)$.

Although Equations (6-19) and (6-20) are derived for the barrier layer of the passive film, we propose that they can be used to describe the growth and thinning of the whole film, which includes the barrier layer and outer layer. Figure 6-21 shows experimental current density (a) and thickness (b) transients, with the latter being calculated from capacitance measurements, for the growth and thinning of the passive film formed on zirconium in $0.1 \mathrm{M} \mathrm{B}(\mathrm{OH})_{3}+0.001 \mathrm{M} \mathrm{LiOH}$ at $250^{\circ} \mathrm{C}$ and 62 bar upon stepping the applied voltage over the cycle $0.30 \mathrm{~V}_{\text {she }} \rightarrow 0.90 \mathrm{~V}_{\text {she }} \rightarrow 0.60 \mathrm{~V}_{\text {she. }}$. When stepping the potential in the positive direction from $0.30 \mathrm{~V}_{\text {she }}$ to $0.90 \mathrm{~V}_{\text {she, }}$, the current density increased very sharply, and then relaxed to a lower value before passing through a maximum followed by a gradual rise. Corresponding to this change in current density, the film thickness increased very rapidly. Upon stepping from $0.90 \mathrm{~V}_{\text {she }} \rightarrow 0.60 \mathrm{~V}_{\text {she, }}$ the current was observed to sharply decrease, followed by a slow rise, while the film thickness decreased at a constant rate $(\approx 0.018 \mathrm{~nm} / \mathrm{s})$ for a short time followed by a gradual decrease in the rate of thinning toward a new steady state.

In order to form a hydrogenated environment that is typical of PWR primary coolant circuits, $45 \mathrm{~cm}^{3}$ (STP) $/ \mathrm{kg}$ hydrogen was added to the $0.1 \mathrm{M} \mathrm{B}(\mathrm{OH})_{3}+0.001 \mathrm{M}$ 
LiOH solution. The hydrogen content in the solution was adjusted by bubbling ultra-high purity hydrogen gas at an appropriate pressure through the solution in the ambient temperature storage tank (reservoir) prior to (overnight) and during the feeding of the solution into the autoclave. Current density and film thickness transients were measured by stepping the potential from open circuit potential $-0.45 \mathrm{~V}_{\text {she }}$ to $0.48 \mathrm{~V}_{\text {she }}$ followed by a step to $0.08 \mathrm{~V}_{\text {she }}$ (Fig. 6-22). When the potential was stepped to $0.48 \mathrm{~V}_{\text {she }}$ from open circuit potential $-0.45 \mathrm{~V}_{\text {she }}$, which resulted in thickening of the film, the current relaxed toward a steady state and the film thickness increased monotonically toward the same steady state. Stepping the potential to the lower value of $0.08 \mathrm{~V}_{\text {she }}$ resulted in a sharp reduction in the current density followed by a relaxation toward a new steady state. The film immediately thins in a linear manner with time $(\mathrm{dL} / \mathrm{dt}=-0.003 \mathrm{~nm} / \mathrm{s})$ and then passes through a minimum as it evolves toward the new steady state.

The above observations are generally in agreement with the rate law afforded by the Point Defect Model. The initial increase in film thickness and current density on stepping the potential from $0.30 \mathrm{~V}_{\text {she }}$ to $0.90 \mathrm{~V}_{\text {she }}$ is due to film growth at the metal/film interface [Reaction (3) in Fig. 6-20]. When stepping the potential in the positive direction, only part $(\alpha \Delta \mathrm{V})$ of the overall potential appears as an additional potential drop across the film (barrier layer)/solution (f/s) interface, whereas an additional potential drop of $(1-\alpha) \Delta \mathrm{V}$ appears across the metal/film $(\mathrm{m} / \mathrm{f})$ interface, where $\alpha$ is the polarizability of the $\mathrm{f} / \mathrm{s}$ interface [2]. The additional potential drop at the $\mathrm{m} / \mathrm{f}$ interface enhances the rate of production of oxygen or hydrogen vacancies, thereby leading to enhanced growth of the film into the metal. If the film dissolution reaction is not an electrochemical process (as in the case of barrier layer oxide formation on zirconium) under BWR environmental conditions, the additional potential drop at the f/s interface (i.e., $\alpha \Delta \mathrm{V}$ ) does not enhance the rate of film dissolution, so that the film thickens. As the film becomes thicker, the voltage drop across the $\mathrm{m} / \mathrm{f}$ interface decreases, because a larger fraction of the potential drop appears across the film, and hence the rate of film growth also decreases, as does the growth current, with $d L / d t$ eventually becoming zero. We see that, upon stepping the potential in the positive direction, particularly at high potentials, the film initially thickens very rapidly and is consistent with the prediction of the PDM [23].

Contrariwise, when the potential is stepped in a negative direction from $0.90 \mathrm{~V}_{\text {she }}$ to $0.60 \mathrm{~V}_{\text {she }}$ or from $0.48 \mathrm{~V}_{\text {she }}$ to $0.08 \mathrm{~V}_{\text {she }}$ [negative $(1-\alpha) \Delta \mathrm{V}$ ], the kinetics of dissolution of the barrier oxide layer at the barrier layer/solution interface control the rate of thinning of the passive film. For negative potential steps, the first term in Equation (6-18) becomes very small and the film thickness is predicted to decrease at a constant rate at short times as shown in Figure 6-21(b) and Figure 6-22(b) due to the film formation process at the metal/film interface shut down; this phenomenon has also been observed for tungsten [23]. The dissolution rate is $\mathrm{c} \approx 0.018 \mathrm{~nm} / \mathrm{s}$ when stepping the potential from $0.90 \mathrm{~V}_{\text {she }}$ to $0.60 \mathrm{~V}_{\text {she }}$ in the solution without hydrogen, while the dissolution rate is $\mathrm{c} \approx 0.003 \mathrm{~nm} / \mathrm{s}$ for stepping the potential from $0.48 \mathrm{~V}_{\text {she }}$ to $0.08 \mathrm{~V}_{\text {she }}$ in hydrogenated solution. Given the approximate nature of the estimated dissolution rate it is not clear whether this difference is significant. However, as the film thins, the potential drop across the film decreases, due to the constant field strength, and the additional potential drop occurs across the $\mathrm{m} / \mathrm{f}$ interface. This causes Reaction (3) in Fig. 6-20, to progressively increase in rate until the 
rate matches the dissolution rate [Reaction (7) in Fig. 6-20], and a new steady state is achieved. The initial linear variation of thickness with time corresponds to the rate of film growth, [Reaction (3) in Fig. 6-20] being negligible compared with the rate of dissolution. The 12-hour period used in this study for film thinning is not sufficiently long for the system to reach a steady state, but we can see that the thinning rate reduces with time and will reach steady state at some point. Note that the sudden drop in film thickness in Figure 6-21 (b) is an experimental artifact due to discharging of the space charge layer in the film. Both the film growth rate and the dissolution rate of the passive film formed in the hydrogenated solution are slower than those in the solution without hydrogen.
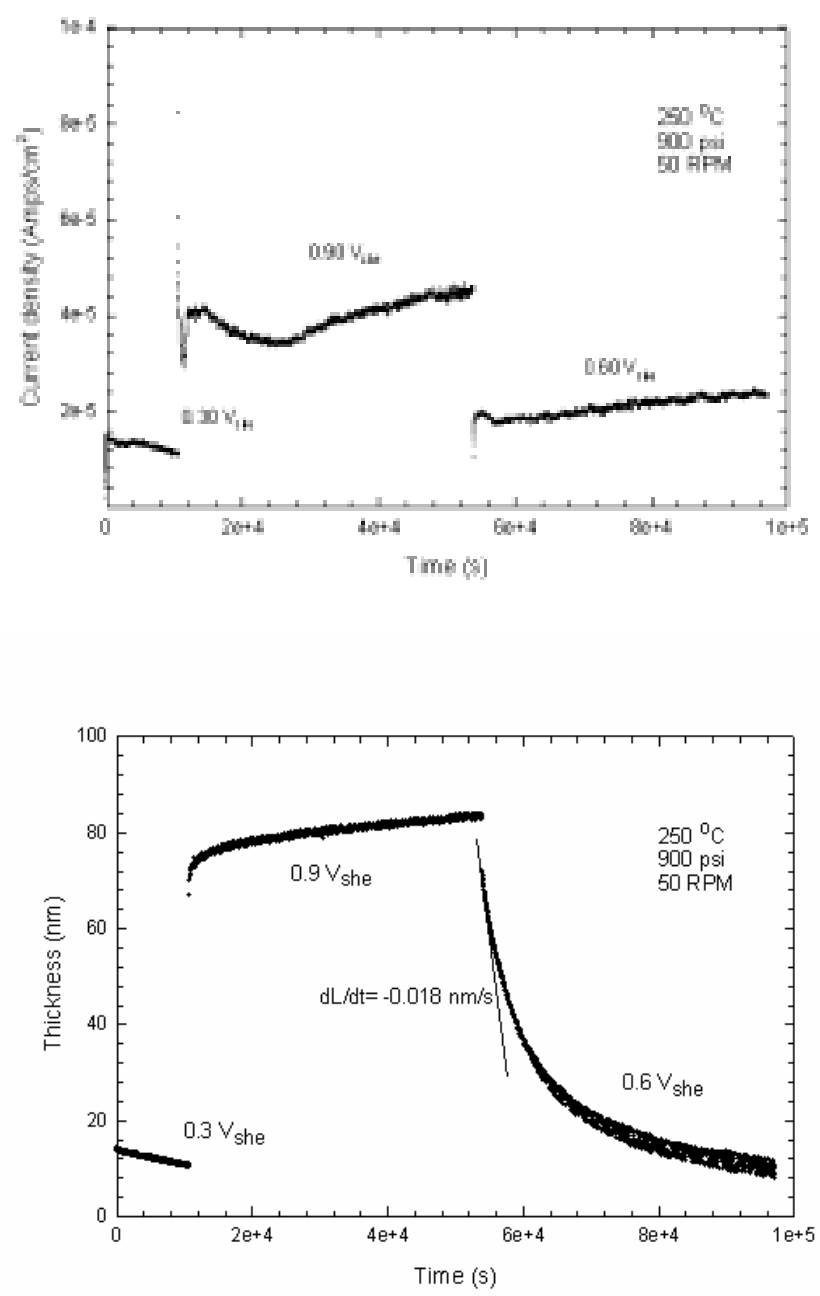

Figure 6-21. Experimental transients in current density and film thickness for the growth and thinning of the passive film on zirconium in $0.1 \mathrm{M} \mathrm{B}(\mathrm{OH})_{3}$ and $0.001 \mathrm{M} \mathrm{LiOH}$ solution at $250{ }^{\circ} \mathrm{C}$ and 62 bar under dehydrogenated conditions upon stepping the potential from $0.30 \mathrm{~V}_{\text {she }} \rightarrow 0.90 \mathrm{~V}_{\text {she }} \rightarrow 0.60 \mathrm{~V}_{\text {she. }}$ current density, (b) thickness calculated from capacitance $(\mathrm{f}=1 \mathrm{kHz})$. 

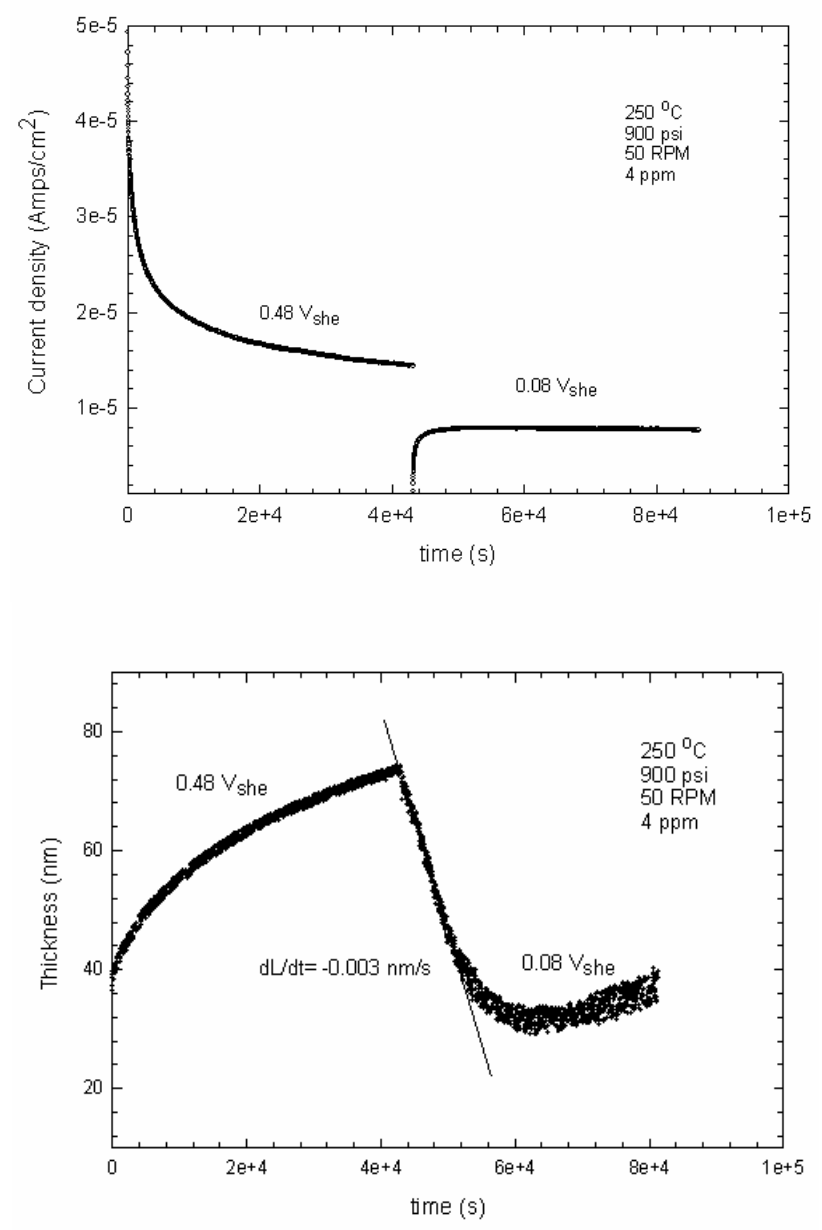

Figure 6-22. Experimental transients in current density and film thickness for the growth and reduction of the passive film on zirconium in $0.1 \mathrm{M} \mathrm{B}(\mathrm{OH})_{3}$ and $0.001 \mathrm{M} \mathrm{LiOH}$ solution containing $45 \mathrm{~cm}^{3}(\mathrm{STP}) / \mathrm{kg}\left(\mathrm{H}_{2} \mathrm{O}\right)$ hydrogen at 250 ${ }^{\circ} \mathrm{C}$ and 62 bar upon stepping the potential from $-0.45 \mathrm{~V}_{\text {she }}$ (open circuit potential) $\rightarrow 0.48 \mathrm{~V}_{\text {she }} \rightarrow 0.08 \mathrm{~V}_{\text {she }}$. (a) current density, (b) thickness calculated from capacitance $(\mathrm{f}=1 \mathrm{kHz})$.

The rotation rate of the flow activating impeller in the cell changes the hydrodynamic conditions of the solution in contact with the sample and hence is expected to play an important role in determining the current transient and changes of film thickness, especially when the system contains hydrogen. For the system without hydrogen (Figure 6-23), when stepping the rotational speed from $100 \mathrm{rpm}$ to $500 \mathrm{rpm}$, the current density increases suddenly but eventually relaxes to a continuation of the current transient that was established at the lower rotation rate. Stepping the rotation rate back to $100 \mathrm{rpm}$ lowers the current density. The corresponding change in the film thickness upon increasing and then decreasing the rotational speed of the impeller is also seen to be small, but it is clearly in the expected direction (higher flow velocity leading to a smaller thickness). The small increase in the current density and the small decrease in the film 
thickness that are observed for the system without hydrogen upon cycling the rotational speed of the flow activating impeller can be explained by the Point Defect Model. The high rotational rate causes a high flow rate at the film/solution interface, resulting in a higher film dissolution rate (increase in the standard rate constant $k_{7}^{0}$ ) while the growth rate at the interface of metal/film interface (described by the standard rate constant $k_{3}^{0}$ ) does not change. These conditions cause the film to thin and result in a slightly raised current density compared to $100 \mathrm{rpm}$. In the hydrogenated system (Fig. 6-24), however, the changes in the current density, in particular, and, to a lesser extent, the change in the film thickness resulting from the change in flow conditions are much more pronounced than the corresponding changes observed in the non-hydrogenated system. It is possible that the difference between the non-hydrogenated and the hydrogenated systems arises from the oxidation of molecular hydrogen $\left(\mathrm{H}_{2}=2 \mathrm{H}^{+}+2 e^{-}\right)$, such that when the rotational speed of the impeller is increased the flux of hydrogen to the surface is also increased and hence so is the hydrogen oxidation current. Superimposition of the redox current on the film growth current would, therefore, account for the more pronounced response to changes in flow conditions observed in the hydrogenated environment. We stress, however, that the observed effects are very small, as can be seen by plotting the currents on a common scale (Fig. 6-25). The smallness of the effect also argues that the electronic conductivity of the passive film on zirconium is also very small; a finding that is consistent with zirconium being a valve metal.

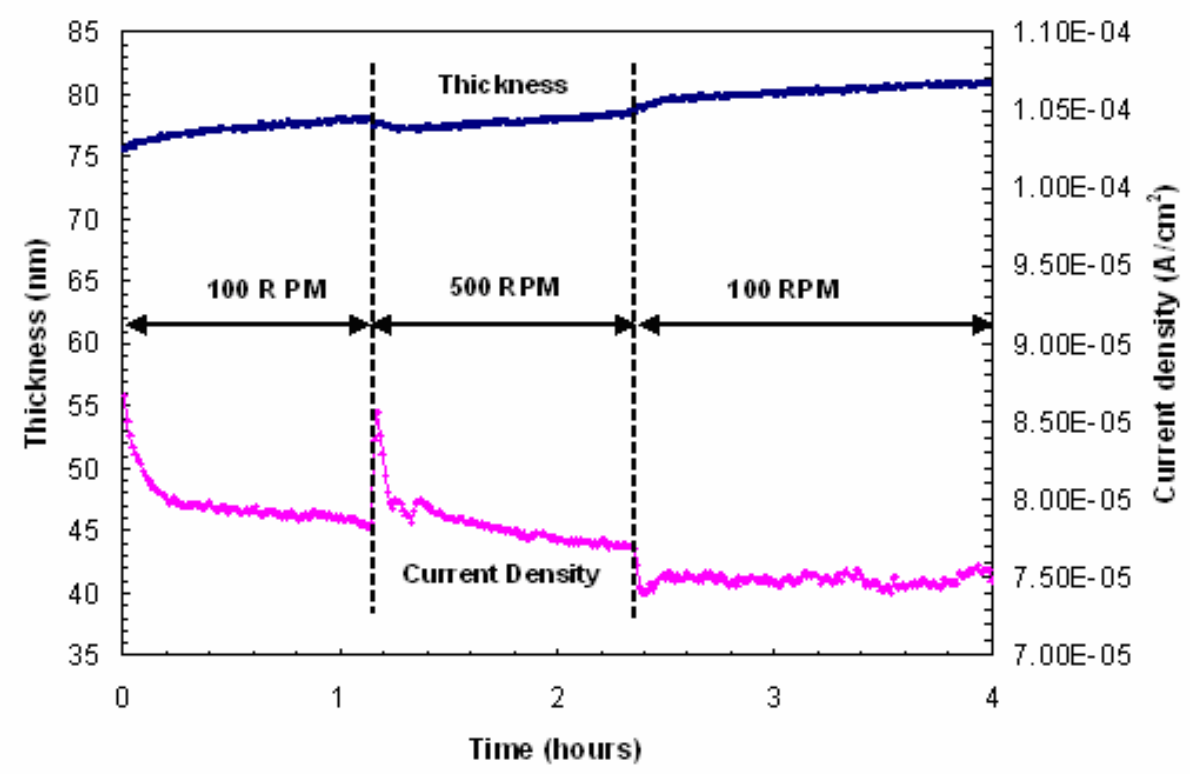

Figure 6-23. The effect of rotation rate on the changes of current density, and film thickness calculated from capacitance measurements of passive film formed at $0.30 \mathrm{~V}_{\text {she }}$ that formed in $0.1 \mathrm{M} \mathrm{B}(\mathrm{OH})_{3}+0.001 \mathrm{M} \mathrm{LiOH}$ solution. $\mathrm{T}=250^{\circ} \mathrm{C}$ and $\mathrm{p}=62 \mathrm{bar}$. 


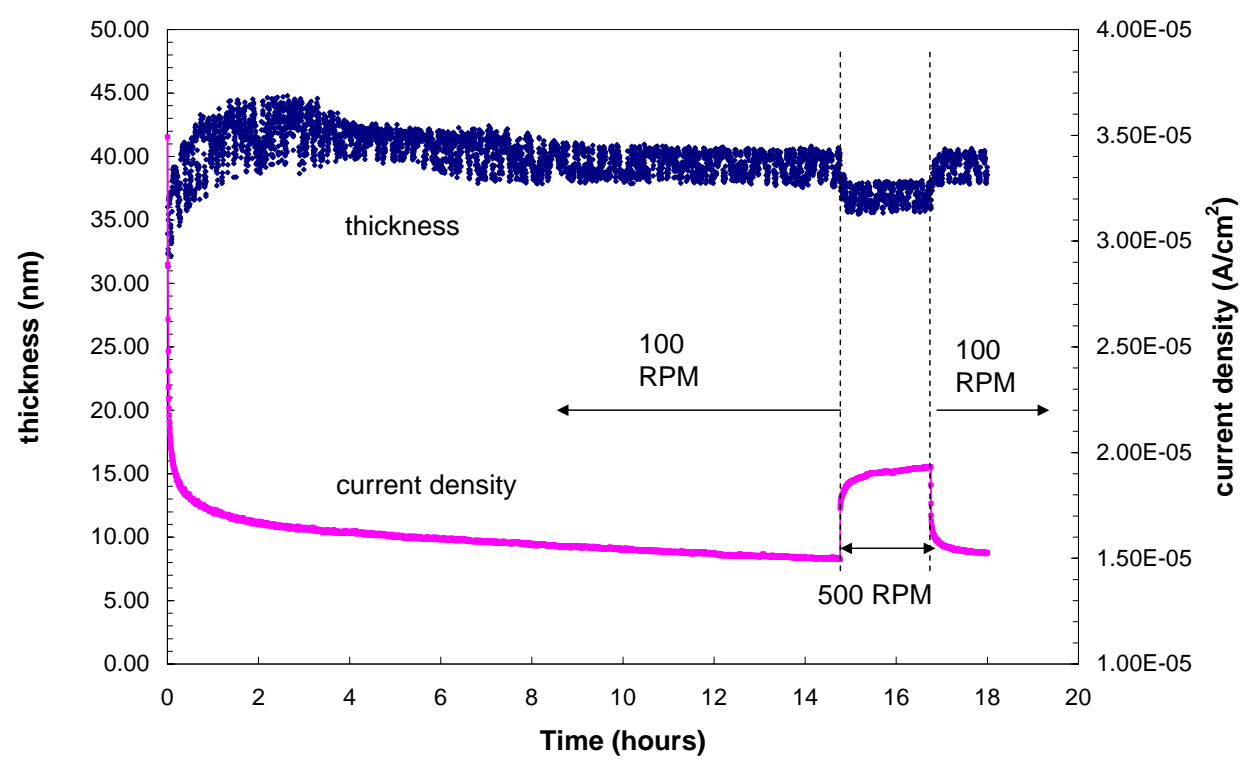

Figure 6-24. The effect of rotation rate on the changes of current density and film thickness calculated from capacitance measurements of passive film formed at $0.38 \mathrm{~V}_{\text {she }}$ in $0.1 \mathrm{M} \mathrm{B}(\mathrm{OH})_{3}+0.001 \mathrm{M} \mathrm{LiOH}$ solution containing 4 ppm hydrogen. $\mathrm{T}=250^{\circ} \mathrm{C}$ and $\mathrm{p}=62 \mathrm{bar}$.

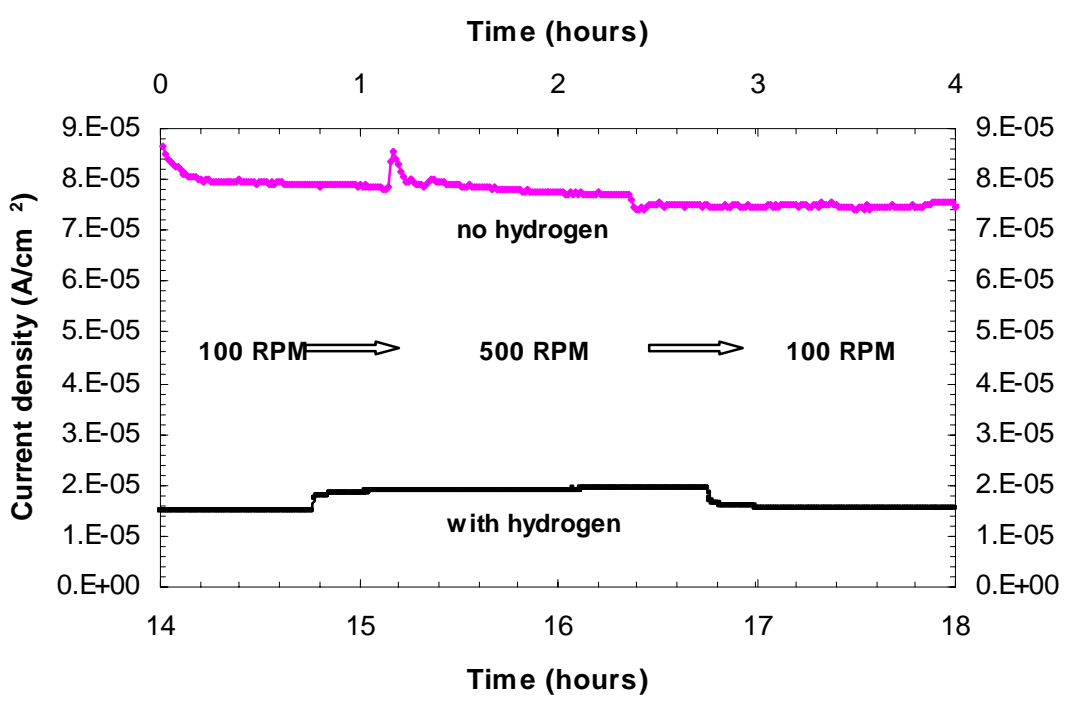

Figure 6-25. Comparison of the current density change with the rotation rate from $100 \mathrm{rpm} \rightarrow 500 \mathrm{rpm} \rightarrow 100 \mathrm{rpm}$ for system with and without hydrogen. The system contains hydrogen shows electron charge transfer phenomenon. 
The findings of this part of work on the transients are summarized as follows:

1) Current density and passive film thickness transients observed on zirconium in $0.1 \mathrm{M}$ boric acid and $0.001 \mathrm{M}$ lithium hydroxide solution at $250^{\circ} \mathrm{C}$ and $62 \mathrm{bar}$ are generally consistent with the predictions of the Point Defect Model.

2) When stepping the potential in the positive direction, the part of potential drop at the metal/film $(\mathrm{m} / \mathrm{f})$ interface enhances the rate of production of oxygen or hydrogen vacancies, thereby leading to enhanced growth of the film into the metal. The current density is observed to increase very rapidly and then to relax to a lower value.

3) Upon stepping the potential in the negative direction, the film thickness initially decreases at a constant rate at short times due to the film formation process at the metal/film interface shut down. However, as the film thins, the potential drop across the film decreases, due to the constant field strength, and the additional potential drop occurs across the $\mathrm{m} / \mathrm{f}$ interface. This causes Reaction (3) in Fig. 6-20, to progressively increase in rate until the rate matches the dissolution rate, Reaction (7), and a new steady state is achieved. The initial linear variation of thickness with time corresponds to the rate of film growth, Reaction (3) in Fig. 6-20, being negligible compared with the rate of dissolution. Both the film growth rate and the dissolution rate of the passive film formed in the hydrogenated solution are slower than those in the solution without hydrogen.

4) Electron charge transfer (oxidation of $\mathrm{H}_{2}$ ) may explain the effect of rotation rate on the changes of current density and film thickness when the solution contains hydrogen.

\subsection{Second Phase Particles and the Electrochemistry of Zircaloy-4}

Pure zirconium cannot be used in reactor systems due to its mechanical softness and low corrosion resistance in water environments. To improve the properties of zirconium, it is alloyed with small amounts of iron $(\mathrm{Fe})$, chromium $(\mathrm{Cr})$, nickel $(\mathrm{Ni})$, and tin (Sn), etc. Due to the low solubility of some of these elements in the zirconium matrix, second phase particles (SPP) are formed together with zirconium. The most often found second-phase particle is $\mathrm{Zr}(\mathrm{Fe}, \mathrm{Cr})_{2}$ in Zircaloy-4. Because of the relatively small amount of alloying elements in the matrix, the volume fraction of the second-phase particles is very small $(0.2 \%-0.4 \%)$ [24]. The sizes of these second phase particles might be comparable to the thickness order of the impermeable barrier layer as reported by recent studies $[25,26]$. This similarity in dimension means the corrosion process could be affected by these second phase particles [11, 27, 28]. The influence of precipitate size on the corrosion resistance of Zircaloy-4 has been shown by autoclave nodular corrosion tests [29-33], however, only few studies have been devoted to the electrochemical effects of these zirconium intermetallics on corrosion, especially for the passive behavior. As one of the fundamental aspects of the corrosion resistance of zirconium alloys, passive behavior of zircaloys has not been studied in detail due to the complexity and difficulties in doing measurements in high temperature and high pressure environments. 


\subsubsection{Characterization of Materials}

The Zircaloy-4 samples used in this study were supplied by Western Zirconium in Ogden, UT. The composition is determined by Electron Probe Micro Analysis (EPMA), as shown in Table 6-1. Zirconium has two crystallographic structures, an $\alpha$-phase (hexagonal close-packed structure) and a $\beta$-phase (body centered cubic). The phase transformation of pure zirconium from the $\alpha$-phase to the high temperature $\beta$-phase occurs at $863 \pm 5^{\circ} \mathrm{C}$ and the melting temperature is $1852 \pm 2^{\circ} \mathrm{C}$. The microstructures of zircaloys depend on heat treatment. Generally in the re-crystallized condition, it consists of equi-axed grains ( $\alpha$-phase) with second phase particles (SPP) found both in the grain boundaries and inside the grains. During $\beta$-quenching, Zircaloy-4 transforms from the $\beta$ phase of the BCC structure to the $\alpha$-phase of the HCP structure. But the $\alpha$-phase can have different microstructures as shown in the $\beta$-quenched and $\alpha$-annealed Zircaloy- 4 samples. Cold working and $\alpha$-annealing after $\beta$-quenching, as generally employed in the fabrication process of Zircaloy-4, will result in the growth and redistribution of secondphase particles.

Table 6-1: Chemical specification for Zircaloy-4 samples determined by Electron Probe Micro Analysis (EPMA), in wt $\%$

\begin{tabular}{|c|c|c|c|c|}
\hline Material & Sn & Ni & Fe & Cr \\
\hline $\mathbf{Z r}-\mathbf{4}$ & 1.36 & 0 & 0.19 & 0.10 \\
\hline
\end{tabular}

All heat treatments were performed in the laboratory using the as-received Zircaloy-4 rod as the starting material. The previous heating history was unknown. The Zircaloy-4 rod was put into a preheated furnace and kept at $1074{ }^{\circ} \mathrm{C}$ in $\beta$-phase for 7 minutes to completely dissolve the precipitates and put the alloying elements back into solution in the matrix. After the heat treatment, the rod was quenched in the water down to room temperature. Half of the $\beta$-quenched rod was further annealed at $750^{\circ} \mathrm{C}$ for 2 hours. Cylinder samples with $4.49 \mathrm{~cm}^{2}$ exposed surface area were machined from the asreceived, $\beta$-quenched and $\alpha$-annealed Zircaloy- 4 rods. Here the heat treatment is used to control the size and density of second phase particles in the Zircaloy-4 samples since precipitate size depends on the heat treatments history. Various cumulative annealing parameters (CAP) have been proposed for this purpose in the literature, based on recrystallization activation energy for corrosion behavior. It is believed that the growth of these precipitates during the various stages of alloy processing sequence will involve Ostwald ripening due to the rapid precipitation of iron and chromium in Zircaloy-4 [34]. The first stage of Ostwald ripening involves a nucleation period, followed by growth of the nuclei as the matrix supersaturation decreases. During the second state, the system tends to minimize its free energy by reducing the total precipitate/matrix interface area. This is achieved by the dissolution of the smallest particles and the growth of the largest one. The cumulative annealing parameters is used to compare the total amount of annealing from different combinations of annealing time and temperature, i.e., 


$$
C A P=\sum A_{i}=\sum t_{i} e^{\left(-\frac{Q}{R T_{i}}\right)}
$$

Where $\mathrm{Ai}$ is the annealing step at $\mathrm{i}, \mathrm{Q}$ is the activation energy (eV/atom), $\mathrm{R}$ is the Boltzmann constant $\left(8.62 * 10^{-5} \mathrm{eV} / \mathrm{K}\right)$, Ti is the annealing temperature $(\mathrm{K})$ at step $\mathrm{i}$, and ti is the time spent at that temperature. The coarsening kinetics has been shown to be second order with an activation energy of $\mathrm{Q} / \mathrm{R}=18700 \mathrm{~K}$ [34]. The quench procedure is not instantaneous so there will inevitably be some precipitate growth during the time it takes for the material to cool down from the beta plus alpha phase formation temperature of $863{ }^{\circ} \mathrm{C}$ to room temperature. The average precipitate diameter can be estimated from CAP value as shown in Fig. 6-26 and the average diameter of second phase particles in the $\beta$-quenched sample will be less than $100 \mathrm{~nm}$ while it is around $150 \mathrm{~nm}$ for $\alpha$-annealed Zircaloy-4 samples.

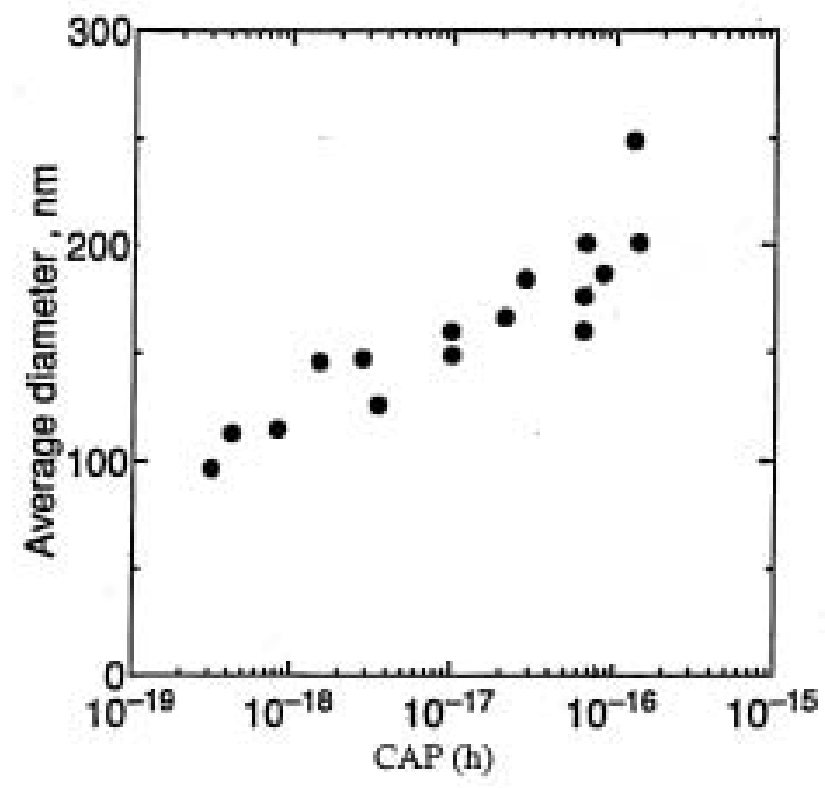

Fig. 6-26: Average precipitate diameter versus CAP in Zircaloy-4 [20].

The microstructures of the as-received, $\beta$-quenched and $\alpha$-annealed Zircaloy- 4 samples were examined by a polarized optical microscopy on the swab etched specimens. The etching was done by using a mixed solution of $20 \mathrm{ml}$ glycerol, $20 \mathrm{ml} 70 \%$ nitric acid and $2 \sim 3 \mathrm{ml} \mathrm{48 \%}$ hydrofluoric acid after mechanical polishing to a mirror finish [35]. The specimens were first mechanically polished with 400, 800 and 1200 grit $\mathrm{SiC}$ paper, and then further polished with $3 \mu \mathrm{m}$ and $1 \mu \mathrm{m}$ diamond paste. The specimen was etched by rubbing for about $10 \sim 15$ second with cotton wool soaked in the solution. For the asreceived $\mathrm{Zr}-4$ sample, a mixed solution of $45 \mathrm{vol} \%$ distilled water, $45 \mathrm{vol} \%$ nitric acid and $10 \mathrm{vol} \%$ hydrofluoric acid was used for better observation result. After etching, specimens were anodized at $15 \mathrm{~V}$ dc for 10-15 seconds in the anodization solution consisting of $60 \mathrm{~mL}$ absolute ethanol, $35 \mathrm{~mL} \mathrm{H} \mathrm{H}_{2} \mathrm{O}, 20 \mathrm{~mL}$ glycerine, $10 \mathrm{~mL} \mathrm{85 \%} \mathrm{lactic}$ 
acid, $5 \mathrm{~mL} 85 \% \mathrm{H}_{3} \mathrm{PO}_{4}$, and $2 \mathrm{~g}$ citric acid [35]. The typical microstructure for the asreceived Zircaloy-4 specimen with equi-axed $\alpha$ grains is shown in Fig. 6-27. When the as-received Zircaloy- 4 specimen was heated to $1074^{\circ} \mathrm{C}$ for 7 minutes and quenched in the water, the fine $\alpha$ grains with parallel or cross needle shapes appeared in the $\beta$-quenched sample, as seen in Fig. 6-28. If the $\beta$-quenched sample is further annealed in $750^{\circ} \mathrm{C}$ for 2 hours, a much coarser $\alpha$ needle pattern is exhibited in the $\alpha$-annealed sample, as shown in Fig. 6-29.

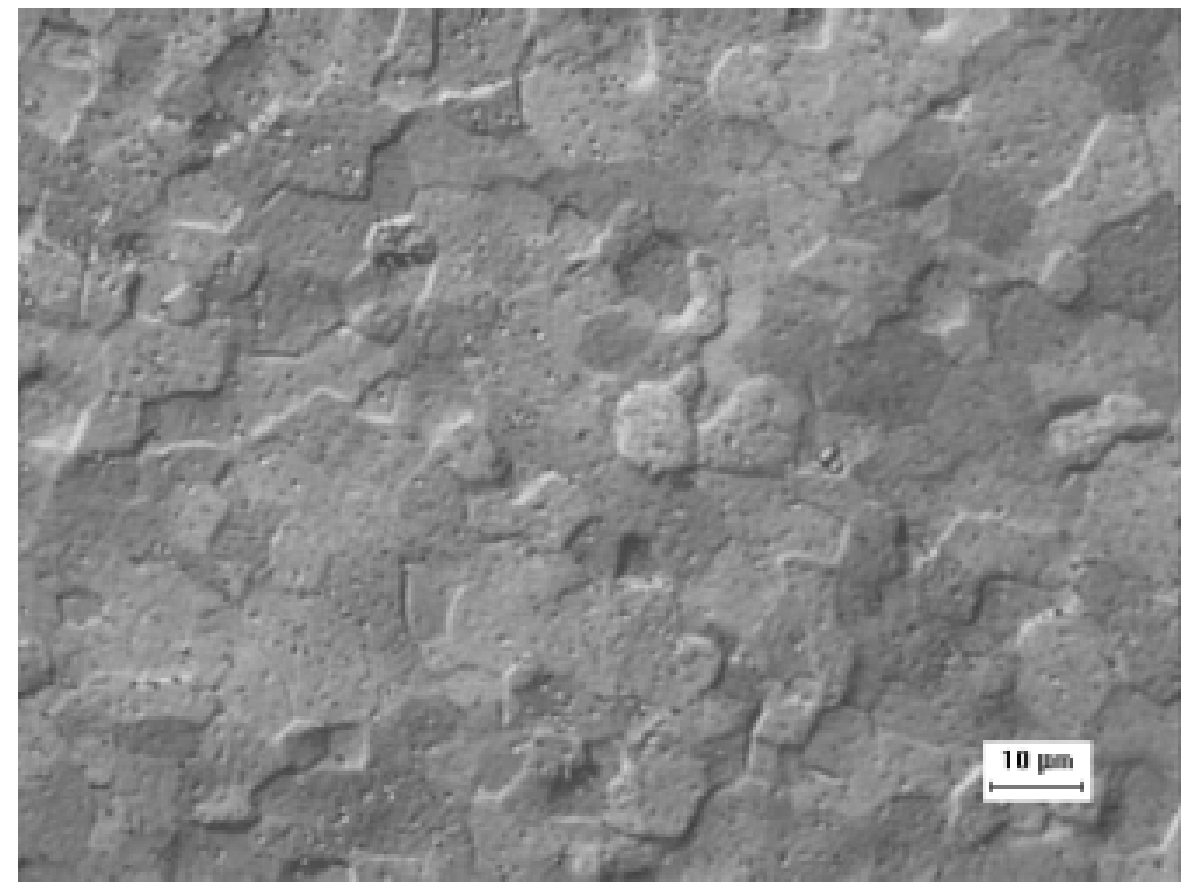

Figure 6-27: As-received Zr-4 sample showing equiaxed $\alpha$ grain structure. Swab etched and anodized at $15 \mathrm{~V}$. Polarized light. 500×. 


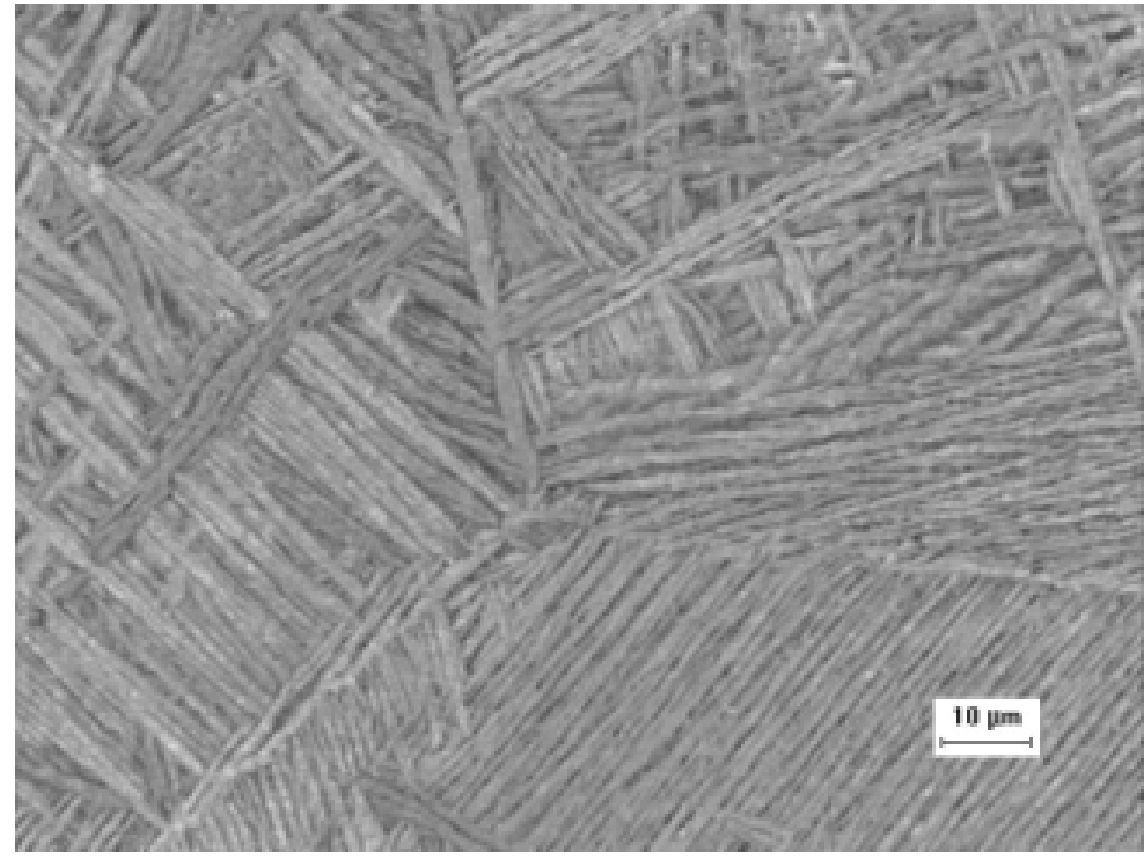

Figure 6-28: $\beta$-quenched $\mathrm{Zr}-4$ sample showing $\alpha$ needles in a parallel or cross pattern. Swab etched and anodized at 15 V. Polarized light. 500×

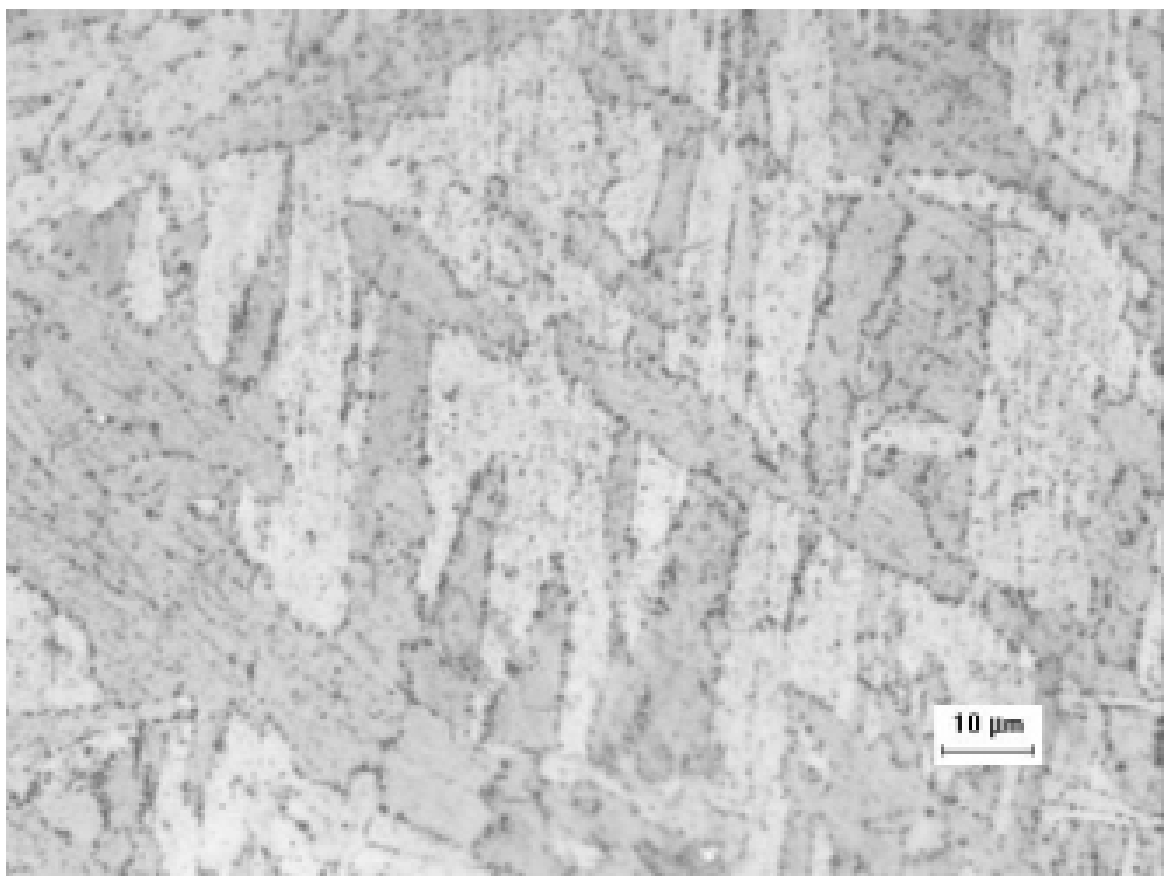

Figure 6-29: $\alpha$-annealed $\mathrm{Zr}-4$ sample showing much coarser $\alpha$ needles in a parallel or cross pattern. Swab etched and anodized at $15 \mathrm{~V}$. Polarized light. $500 \times$ 
In order to understand the effects of second phase particles on the properties of the zirconium passive films, Quanta 200 scanning electron microscopy (SEM) from FEI with attached energy-dispersive X-ray spectrometry (EDX) was used to determine the size and density of the second phase particles in the specimens. For SEM examination, the different heat treated Zircaloy-4 specimens were first mechanically polished to $1 \mu \mathrm{m}$ with a final etching. For better SEM observations on second phase particles, a mixed solution of $20 \mathrm{ml}$ glycerol, $20 \mathrm{ml} 70 \% \mathrm{HNO}_{3}$ and $2 \sim 3 \mathrm{ml} 48 \% \mathrm{HF}$ solution was used for etching after mechanical polishing to a mirror finish. The specimen was etched by rubbing for about 10 20 seconds with cotton wool soaked in the solution [35].

The etched samples were examined in the Quanta 200 FEI at $20 \mathrm{kV}$ in a low vacuum environment. Table 6-2 summarizes the particle densities and sizes of the second phase particles observed in the different heat treated Zr-4 specimens. The second phase particles were found to be $\mathrm{Zr}(\mathrm{Fe}, \mathrm{Cr})_{2}$ as determined by EDX, as shown in Fig. 6-30. Fig. 6-31 shows the second phase particles observed on the $\alpha$-annealed Zircaloy- 4 sample in the secondary electron imaging mode and backscattering electron imaging mode respectively. The most probable particle size for the second phase particles in the $\alpha$ annealed Zircaloy-4 sample is found to be about $180 \mathrm{~nm}$ which is determined by the XT Docu software, as demonstrated in Fig. 6-32. The second phase particles are mainly located along the grain boundary with a density of $0.5 \mu \mathrm{m}^{-2}$. A larger particle size of 210 $\mathrm{nm}$ with a much lower density of $0.1 \mu \mathrm{m}^{-2}$ is found in the as-received Zircaloy-4 sample (Fig. 6-33). The second phase particle size in the $\beta$-quenched Zircaloy-4 sample is found to be less than $100 \mathrm{~nm}$ and the density is too high to be countable. The sizes of the second phase particles in the different heat treated Zircaloy-4 samples are consistent with the values estimated from the cumulative annealing parameters. Of course, it is more accurate to use size distribution instead of the most possible particle size to describe the second phase particles in the sample, as done by Gros and Wadier [34]. However, in this work, we mainly focus on the effects of second phase particles on the properties of passive films formed. The differences in the size and density of the second phase particles in the Zircaloy-4 samples caused by different heat treatments are large enough for identification of the main factor.

Table 6-2: Second phase particles in Zircaloy-4 observed by SEM

\begin{tabular}{|c|c|c|}
\hline Heat Treated samples & $\begin{array}{c}\text { Particle density } \\
\left(\mu \mathrm{m}^{-2}\right)\end{array}$ & $\begin{array}{c}\text { Most probable } \\
\text { particle size }(\mu \mathrm{m})\end{array}$ \\
\hline$\beta$-quenched & High & $<0.1$ \\
\hline as-received & 0.1 & 0.21 \\
\hline$\alpha$-annealed & 0.5 & 0.18 \\
\hline
\end{tabular}



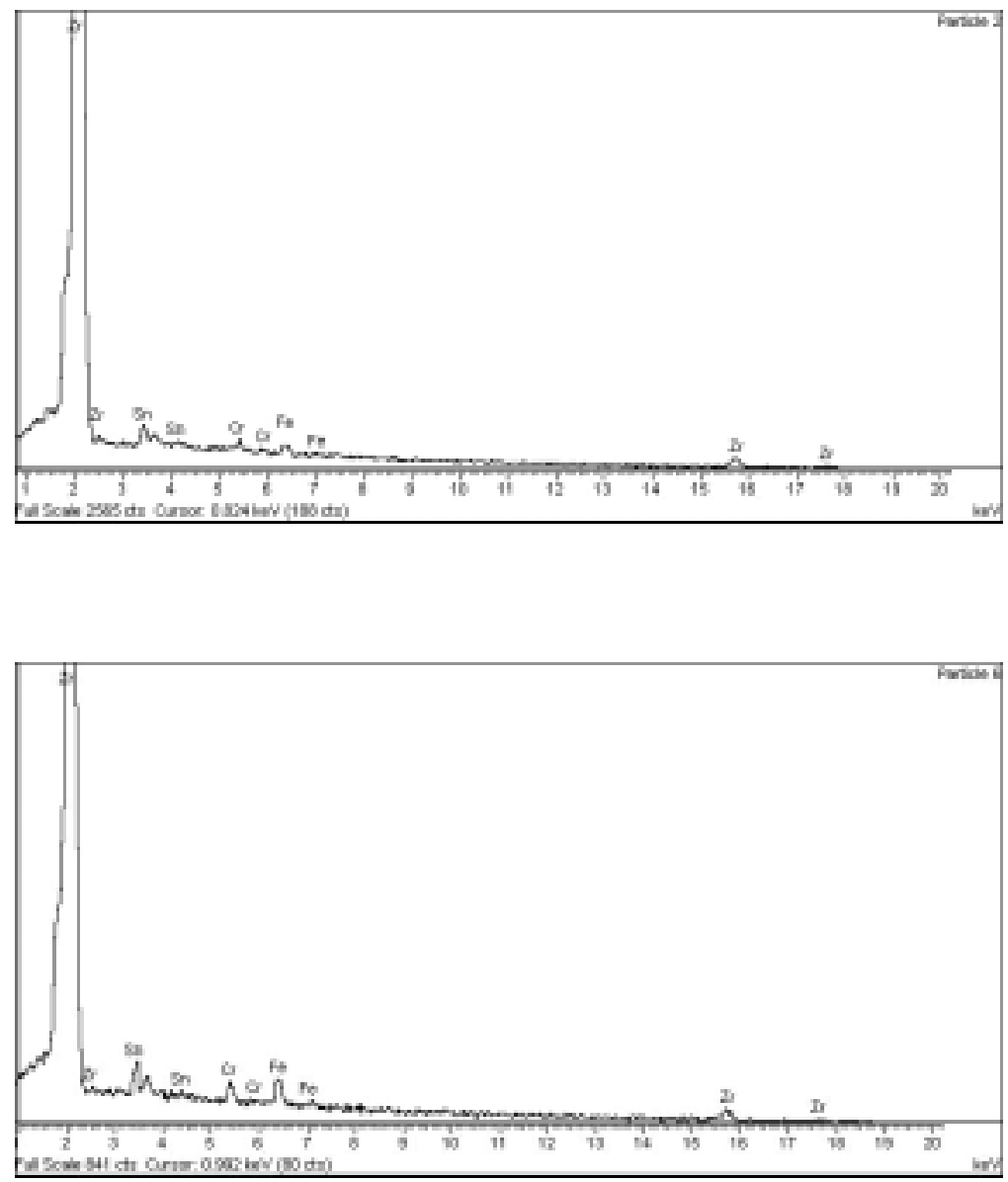

Figure 6-30: The EDX spectra were obtained in the central positions of the randomly picked precipitates of particle 2 and 6 in the $\alpha$-annealed $\mathrm{Zr}-4$ sample. 

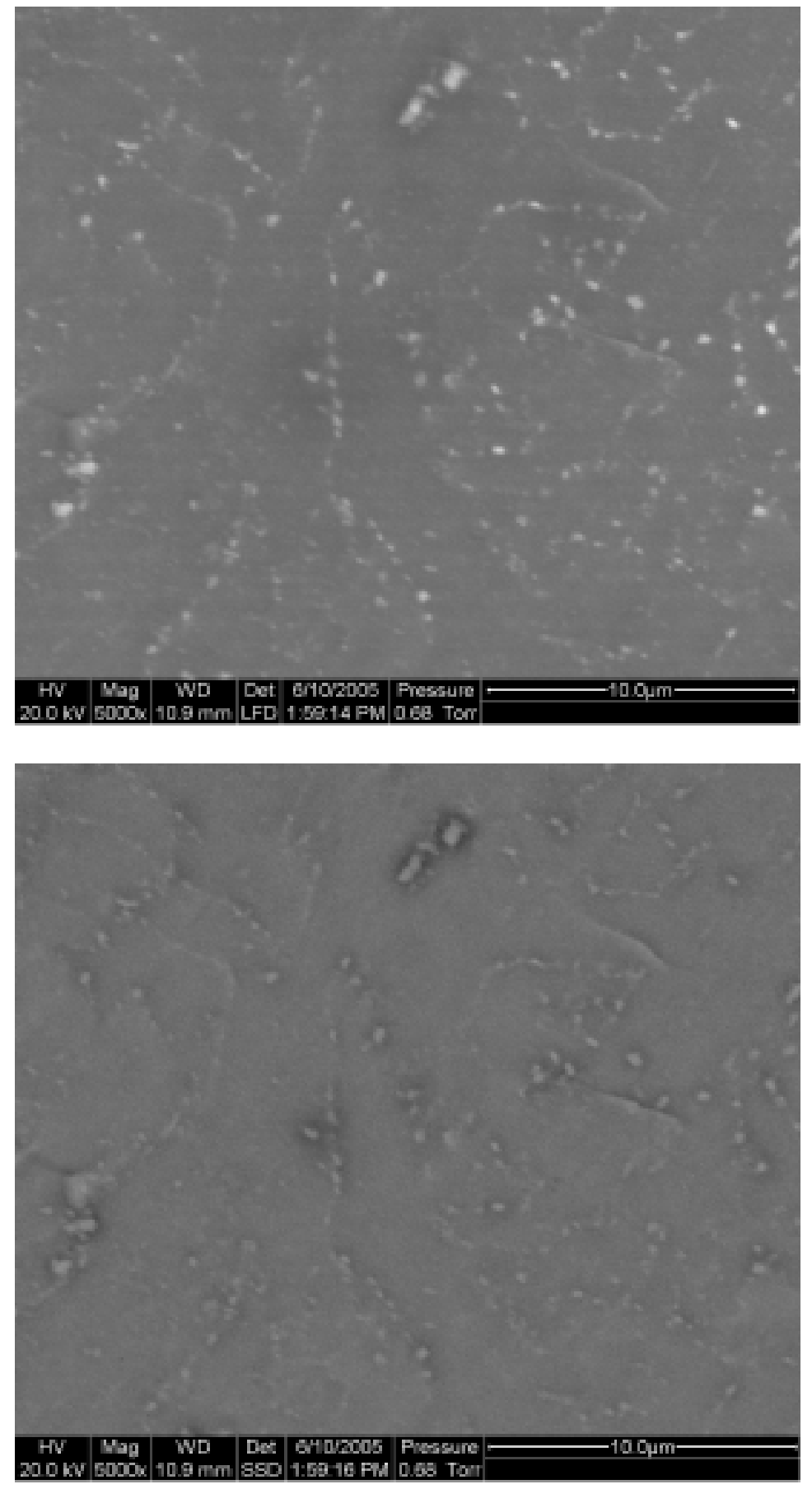

Figure 6-31: SEM observations of second phase particles in the $\alpha$-annealed $\mathrm{Zr}-4$ sample. (upper: Secondary electron imaging mode, down: Backscattering electron imaging mode) 


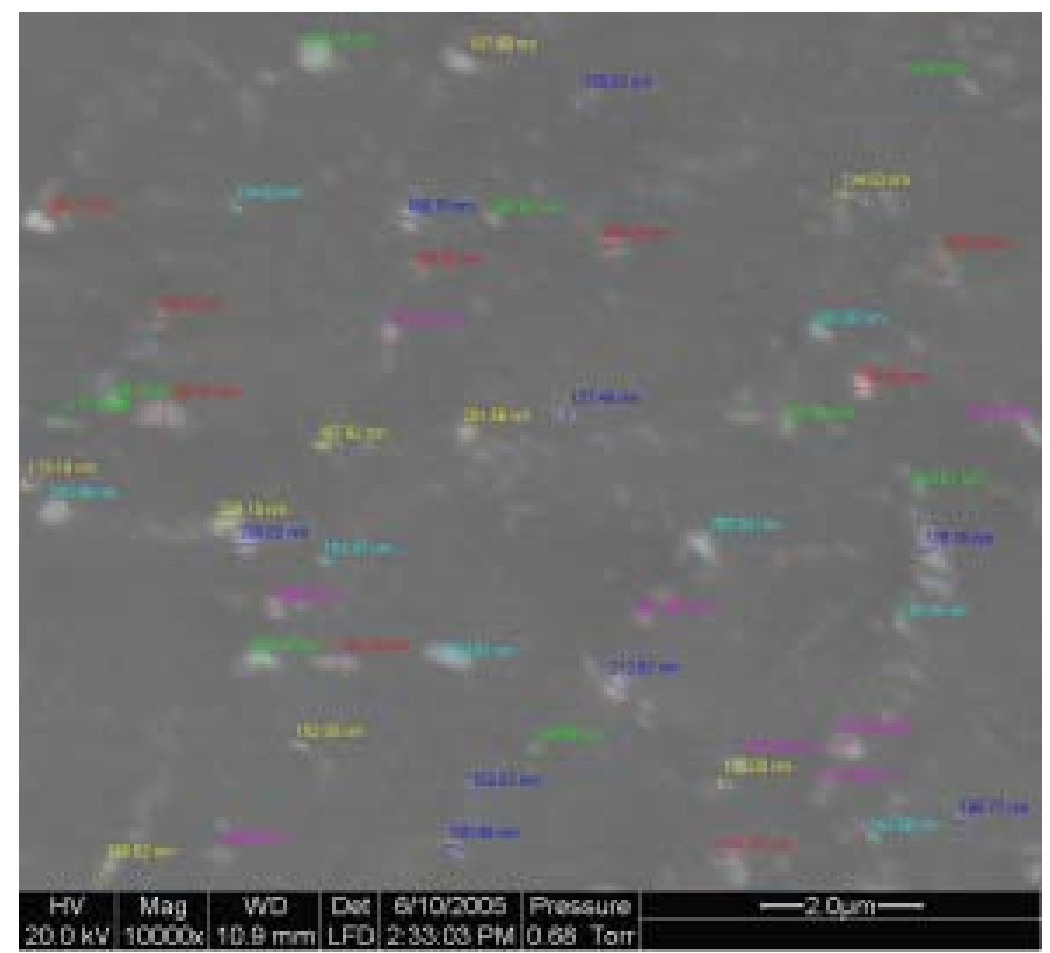

Figure 6-32: The sizes of the second phase particles found in the $\alpha$ - annealed Zircaloy- 4 sample (average particle size $=180 \mathrm{~nm}$ )

\subsubsection{Electrochemistry of Heat Treated Zr-4}

In-situ studies are being carried out by using a controlled hydrodynamic apparatus to examine the effects of second phase particles on the electrochemistry of zirconium passive films formed on the Zircaloy-4 samples with different heat treatment, which eventually changed the size and distribution of the second phase particles in the sample. The working electrode was fabricated from the as-received (AR), $\beta$-quenched (BWQ) and $\alpha$-annealed (BWA) Zircaloy-4 rods with a diameter of $9.8 \mathrm{~mm}$ and an exposed surface area of $4.49 \mathrm{~cm}^{2}$. All samples were braded with 140 grit SiC paper and followed with 400, 800 and 1200 grit $\mathrm{SiC}$ paper. They were further polished with $3 \mu \mathrm{m}$ and $1 \mu \mathrm{m}$ diamond paste. The electrolyte was $0.1 \mathrm{M} \mathrm{B}(\mathrm{OH})_{3}+0.001 \mathrm{M} \mathrm{LiOH}$ with 45 $\mathrm{cm}^{3}(\mathrm{STP}) / \mathrm{kg}\left(\mathrm{H}_{2} \mathrm{O}\right)$ hydrogen. The hydrogen content in the solution was adjusted by bubbling ultra-high purity hydrogen gas at an appropriate pressure through the solution in the storage tank overnight at room temperature before the solution was continuously fed into the autoclave. 

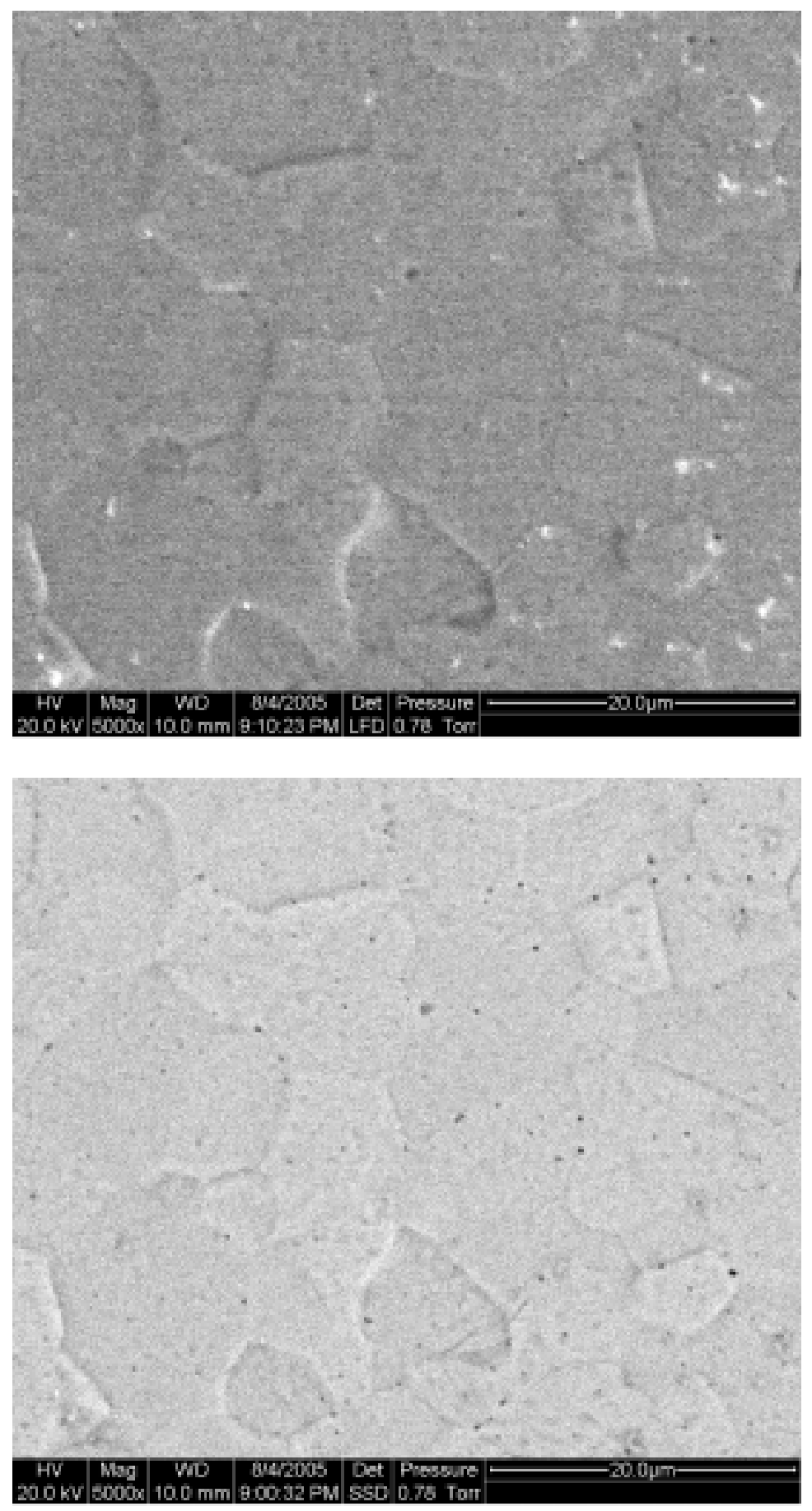

Figure 6-33: SEM observations of second phase particles in the as-received Zr-4 sample. (upper: Secondary electron imaging mode, down: Backscattering electron imaging mode) 


\subsubsection{Polarization Studies}

The polarization curves were recorded by starting the voltage sweep at a rate of 1 $\mathrm{mVs}^{-1}$ from a point $0.2 \mathrm{~V}$ more negative than the zero current potential. Fig. 6-34 shows the comparison of the polarization curves for the as-received, $\beta$-quenched, and $\alpha$ annealed Zircaloy- 4 samples. The polarization curve of the $\beta$-quenched Zircaloy- 4 displays a much more negative zero current potential with the passive region extending from $-0.8 \mathrm{~V}_{\text {she }}$ to $0.1 \mathrm{~V}_{\text {she }}$. The passive region of the $\alpha$-annealed sample is much more positive than the $\beta$-quenched sample ranging from -0.25 to $0.65 \mathrm{~V}_{\text {she }}$ and the passive region is split into two by a spike located around $0.05 \mathrm{~V}_{\text {she. }}$ The passive region of the asreceived Zircaloy- 4 sample is even more positive extending from $-0.2 \mathrm{~V}_{\text {she }}$ to $0.8 \mathrm{~V}_{\text {she. }}$. The $\alpha$-annealed sample has the highest passive current comparing to the other two samples, indicating a lower corrosion resistance, which is consistence with the impedance measurement results. The spike on the $\alpha$-annealed Zircaloy- 4 sample still existed after 10 cyclic voltammetry scannings with a scan rate of $50 \mathrm{mVs}^{-1}$ in the same potential range as the polarization measurements, indicating a slow dissolution rate of the second phase particles in the $\alpha$-annealed sample. The appearance of the spike is believed to be related to the second phase particles, which is confirmed by measured impedance and capacitance as a function of potential.

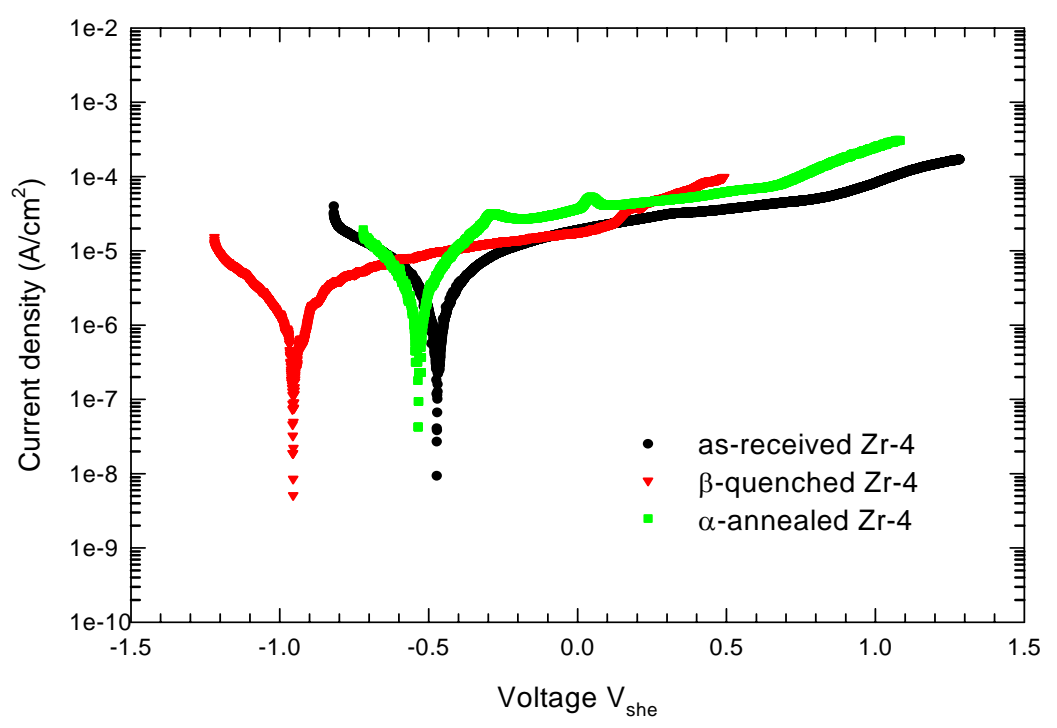

Figure 6-34: Comparison of polarization curves for the as-received, $\beta$ quenched and $\alpha$-annealed $\mathrm{Zr}-4$ samples in $0.1 \mathrm{M} \mathrm{B}(\mathrm{OH})_{3}+0.001 \mathrm{M} \mathrm{LiOH}$ with $45 \mathrm{~cm}^{3}(\mathrm{STP}) / \mathrm{kg}\left(\mathrm{H}_{2} \mathrm{O}\right)$ hydrogen. The hydrogen concentration was maintained in the reservoir by suitably controlling the total gas pressure in the system. Scan rate $=1.0 \mathrm{mV} / \mathrm{s}, \mathrm{T}=250^{\circ} \mathrm{C}$ and $=62 \mathrm{bar}$. 


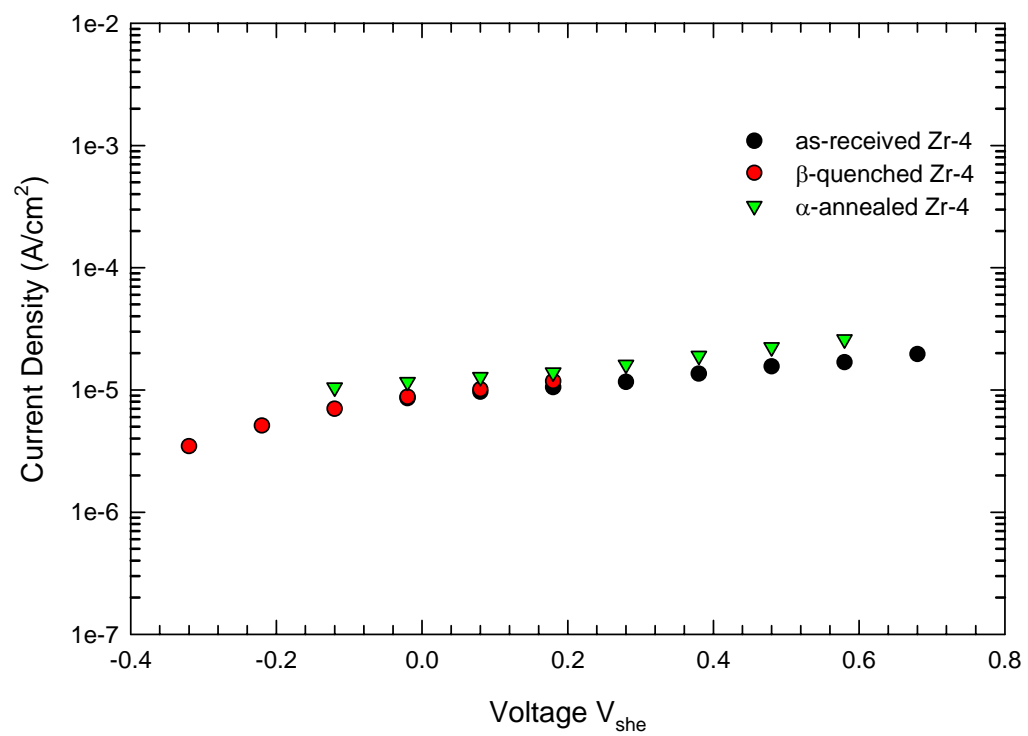

Figure 6-35: The dependence of the steady state current density on the formation potential for the passive films formed on the as-received, $\beta$ quenched and $\alpha$-annealed Zircaloy-4 samples in $0.1 \mathrm{M} \mathrm{B}(\mathrm{OH})_{3}+0.001 \mathrm{M}$ $\mathrm{LiOH}$ solution with $45 \mathrm{~cm}^{3}(\mathrm{STP}) / \mathrm{kg}\left(\mathrm{H}_{2} \mathrm{O}\right)$ hydrogen. The current was recorded after 24 hours film growth at each potential. The potential was changed in the ascending voltage direction. $\mathrm{T}=250{ }^{\circ} \mathrm{C}$ and $\mathrm{P}=62 \mathrm{bar}$.

Potentiostatic experiments were carried out by holding the potential constant at the desired value until the current reached a steady state ( 24 hours). The measurement of the current was made at each potential in the ascending potential direction. Unlike the independence of the logarithm of the steady state current density to the potential for pure zirconium, it exhibits a slight increase with the formation potential (Fig. 6-35), especially for some points in the $\beta$-quenched Zircaloy- 4 sample. This may be due to the oxidation of the alloying elements in the Zircaloy- 4 sample, or it is possible that 24 hours is not sufficient for film stabilization at $250^{\circ} \mathrm{C}$ and the system had not achieved a steady state (defined in the PDM as the state in which the rate of passive film formation at the metal/film interface is equal to the rate of film dissolution).

Same as that done for the pure zirconium, film thickness was calculated from the measured capacitance by using the well-known "parallel plate" expression for the capacitance to estimate the steady state film thickness, $\mathrm{L}_{\mathrm{ss}}$

$$
L=\frac{\varepsilon \varepsilon_{0} A}{C}
$$

All parameters were same as defined earlier. The passive film formed on the $\beta$-quenched sample has the highest film thickness comparing to the passive films formed on the asreceived and $\alpha$-annealed samples (Figure 6-36). The behavior of sudden decrease in the film thickness is observed in all the three samples, with much lower thickness than their neighbors happened at $-0.02 \mathrm{~V}_{\text {she }}$ for $\beta$-quenched sample, $0.18 \mathrm{~V}_{\text {she }}$ for $\alpha$-annealed 
sample and $0.28 \mathrm{~V}_{\text {she }}$ for as-received Zircaloy-4 sample. The sudden change behavior has also been observed in the measured impedance and capacitance as a function of potential. More details will be given later in this section. The passive film thickness is increased with the formation potential if negating the sudden decrease in the above indicated potential points. The film thicknesses are found to be comparable to the sizes of second phase particles and this similarity in dimension means that the corrosion process could be affected by these SPPs. The magnitude of the decrease in the film thickness is found to be related to the size of the second phase particles in the different heat treated samples, i.e., lowest decrease happened in the $\beta$-quenched sample which has the smallest SPP size while largest difference happened in the as-received sample which has the largest SPP size. The sudden decrease in the film thickness may be attributed to the fast dissolution of the second phase particles happened in these potentials. The estimated anodizing constant was $150 \mathrm{~nm} / \mathrm{V}$ for the passive film formed on the $\beta$-quenched Zircaloy- 4 sample in the buffered solution with $45 \mathrm{~cm}^{3}(\mathrm{STP}) / \mathrm{kg}\left(\mathrm{H}_{2} \mathrm{O}\right)$ hydrogen and similar values were obtained for the second passive regions of the other two samples. This value is much greater than the $1.9-2.5 \mathrm{~nm} / \mathrm{V}$ normally found for barrier layers formed on metals and alloys at ambient or near-ambient temperatures. The higher values are attributed to the formation of thick outer layers over thin barrier layers, as explained earlier in the pure zirconium section.

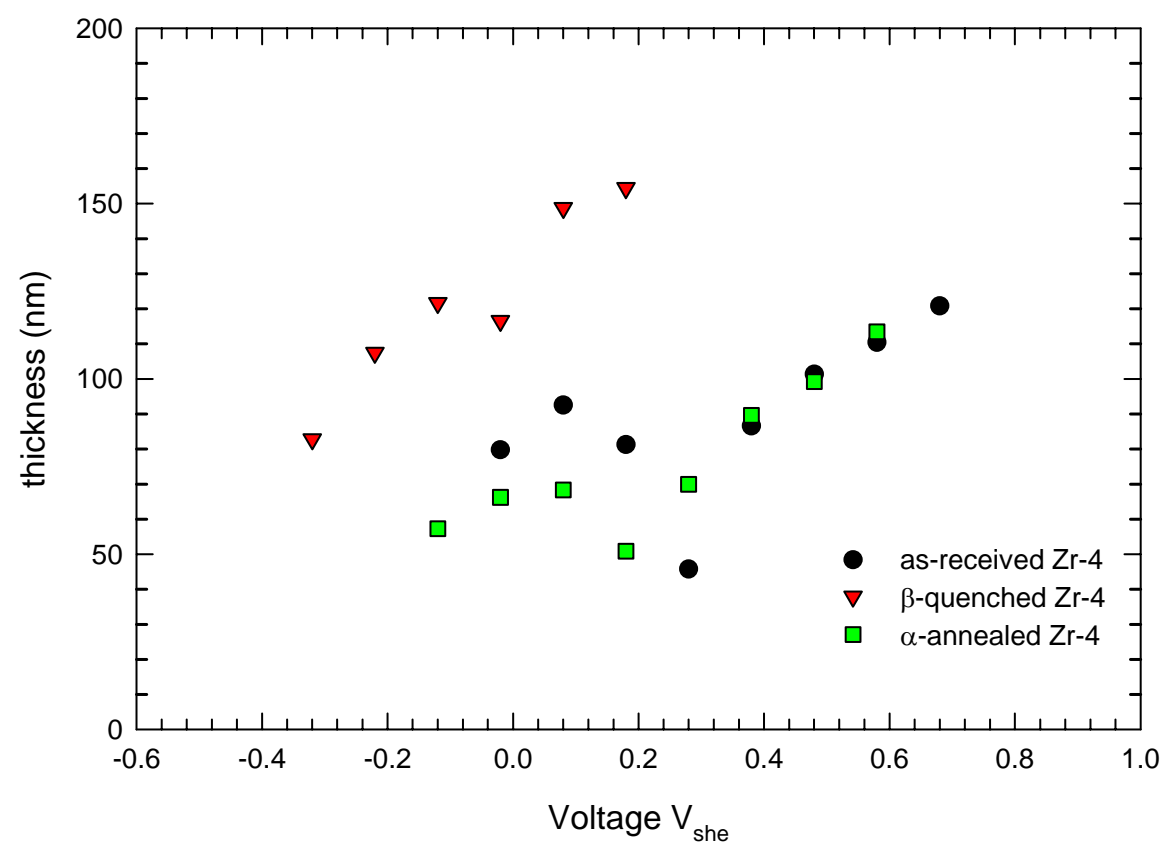

Figure 6-36: Dependence of the steady state film thickness on the applied potentials for the passive films formed on the as-received, $\beta$-quenched and $\alpha$ annealed zircaloy-4 samples in $0.1 \mathrm{M} \mathrm{B}(\mathrm{OH})_{3}+0.001 \mathrm{M} \mathrm{LiOH}$ solution with 45 $\mathrm{cm}^{3}(\mathrm{STP}) / \mathrm{kg}\left(\mathrm{H}_{2} \mathrm{O}\right)$ hydrogen. The potentials were changed from negative to positive direction. Capacitance was measured after holding potential at each step 24 hours for film growth. $\mathrm{T}=250^{\circ} \mathrm{C}$ and $\mathrm{P}=62$ bar. 


\subsubsection{Electrochemical Impedance Spectroscopy}

The corrosion resistance of different heat treated Zircaloy-4 samples was determined by the electrochemical impedance measurements after the growth of passive film reach steady state by holding formation potential constant at each point for 24 hours in the hydrogenated borate buffer solution at $250^{\circ} \mathrm{C}$ and 62 bar. Electrochemical impedance spectra (EIS) for passive films formed on the different heat treated Zircaloy-4 surfaces were measured over a wide range of frequencies (typically $100 \mathrm{kHz}$ to $0.01 \mathrm{~Hz}$ ) as a function of the formation potential across the passive range. The measurements were carried out in the ascending potential direction. Since the stability of the electrochemical system during EIS measurement is critical to obtaining viable data, the quality of the EIS data was checked experimentally by stepping the frequencies from high-to-low and then immediately back from low-to-high, with the impedance being measured at each step to ascertain that the same values were obtained at equivalent frequencies in the two directions. If the system is in the steady state, which means the thickness and current are independent of time, the impedance data should match in the two potential step directions. Impedance data measured in the two step directions were coincident for all the formation potentials. The impedance of the $\beta$-quenched Zircaloy-4 sample (Fig. 6-37) was found to be insensitive to the film formation potential in the passive range, although little deviation is noticed on the Nyquist plots and Bode plots which indicate the relationship to the frequency. However, the impedances of passive films formed on the $\alpha$-annealed $\mathrm{Zr}-4$ sample (Fig. 6-38) at different potential show a clear transient happened at around potential of $0.18 \mathrm{~V}_{\text {she }}$ to $0.28 \mathrm{~V}_{\text {she }}$. The transient in the impedance splits the passive range into two parts. For the impedances in the same passive region, they are independent to the formation potential, with a higher impedance value in the lower potentials and smaller impedance for the higher potentials. The independence also noticed in the Bode plot. Similar two-passive-region behavior is also happened in the as-received Zircaloy-4 sample as shown in Fig. 6-39 with transient happened at potentials $0.28 \mathrm{~V}_{\text {she }}$ to $0.38 \mathrm{~V}_{\text {she }}$. The corresponding film thicknesses (see Fig. 6-36) at these potentials were much lower than their neighbors may indicate a faster dissolution rate. Therefore, second-phase particles are believed to split the passive region into two parts with different corrosion resistance for the as-received and the $\alpha$-annealed samples, although not obvious for the $\beta$-quenched sample. By comparing the impedances for the three different heat treated $\mathrm{Zr}$ 4 samples and neglecting the impedance values in the transient parts (Fig. 6-40), the $\alpha$ quenched $\mathrm{Zr}-4$ sample shows the highest corrosion resistance, while the $\alpha$-annealed sample is the lowest.

\subsubsection{Mott-Schottky Analysis}

An understanding of the crystallographic and electronic defect structures of the passive film on Zircaloy-4 is vital in exploring the underlying mechanisms of oxidation and hydriding of Zircaloy fuel cladding in reactor coolant environments. To identify the principal defect in the passive film, Mott-Schottky (M-S) analysis [2] is being used. In M-S analysis, the capacitance of the passive film is measured at a suitably high frequency as a function of voltage. According to Mott-Schottky theory [20], the space charge capacitance $C_{s c}\left(\mathrm{~F} / \mathrm{cm}^{2}\right)$ of n-type and p-type semiconductor junctions are given by 


$$
\frac{1}{C_{s c}^{2}}=\frac{2}{\varepsilon \varepsilon_{0} q N_{D} A^{2}}\left(E-E_{f b}-\frac{k T}{q}\right) \quad \text { n-type }
$$

and

$$
\frac{1}{C_{s c}^{2}}=\frac{-2}{\varepsilon \varepsilon_{0} q N_{A} A^{2}}\left(E-E_{f b}-\frac{k T}{q}\right) \quad \text { p-type }
$$

respectively. In these expressions, $N_{D} / N_{A}$ is the donor/acceptor concentration $\left(\mathrm{cm}^{-3}\right), \varepsilon_{0}$ is the vacuum permittivity $\left(8.85 \times 10^{-14} \mathrm{~F} / \mathrm{cm}\right), \varepsilon$ is the dielectric constant of the oxide, $V$ is the applied potential, $V_{f b}$ is the flat band potential, $q$ is the electron charge $\left(1.6 \times 10^{-19} \mathrm{C}\right)$, and $k T / q$ is about $45 \mathrm{mV}$ at $250{ }^{\circ} \mathrm{C}$. In addition to confirming the electronic nature of the film, M-S analysis yields the dopant concentration (donor or acceptor) in the film as a function of voltage. The interfacial capacitance $C$ is obtained from $C=-1 / \omega Z$ " . Assuming that the capacitance of the Helmholtz layer can be neglected, the measured capacitance $C$ is equal to the 'space charge' capacitance, $\mathrm{C}_{\mathrm{sc}}$. Accordingly, a $C_{s c}{ }^{-2}$ versus $\mathrm{V}$ plot should be a straight line with a slope that is inversely proportional to the dopant concentration.

The outer layer of the passive film formed on zirconium is found to be much thicker than the barrier layer. The thin barrier layer is a defective phase with semiconductor properties that depend upon the type of defects present while the outer layer is relatively porous and has no appreciable semiconductor properties [6]. Therefore, the measured capacitance cannot be directly approximated as the space charge capacitance; instead, the observed capacitance $C$ can be understood by postulating that it reflects a series combination of a voltage-independent capacitance, $C_{o x}$, due to the outer layer insulting layer, and the space charge capacitance, $C_{b l}$, of the barrier layer, as discussed in the thickness calculation section of this paper.

$$
\frac{1}{C}=\frac{1}{C_{o x}}+\frac{1}{C_{b l}}
$$

It has been shown by de Gryse et al. [21] that the measured capacitance can be related to $C_{o x}$ and the applied voltage by

$$
\frac{1}{C^{2}}=\frac{1}{C_{o x}^{2}}+\frac{2}{\varepsilon \varepsilon_{0} q N_{D}}\left(V-V_{f b}-\frac{k T}{q}\right)
$$

where the notation is same as previously defined. As can be seen from this expression, the slope of the MS-plot is not influenced by the oxide capacitance $C_{o x}$ and can be used to derive reliable values for the donor density, $N_{D}$, from the slope of the measured MS-plot, although the intercept is shifted on the vertical and horizontal axes, thereby yielding a flat band potential that is too negative. Mott-Schottky analysis can be applied to a system of fixed (voltage-independent) total dopant concentration and, hence, $C$ versus $V$ must be measured under the conditions that do not result in a change in the dopant concentration profile of the film. 

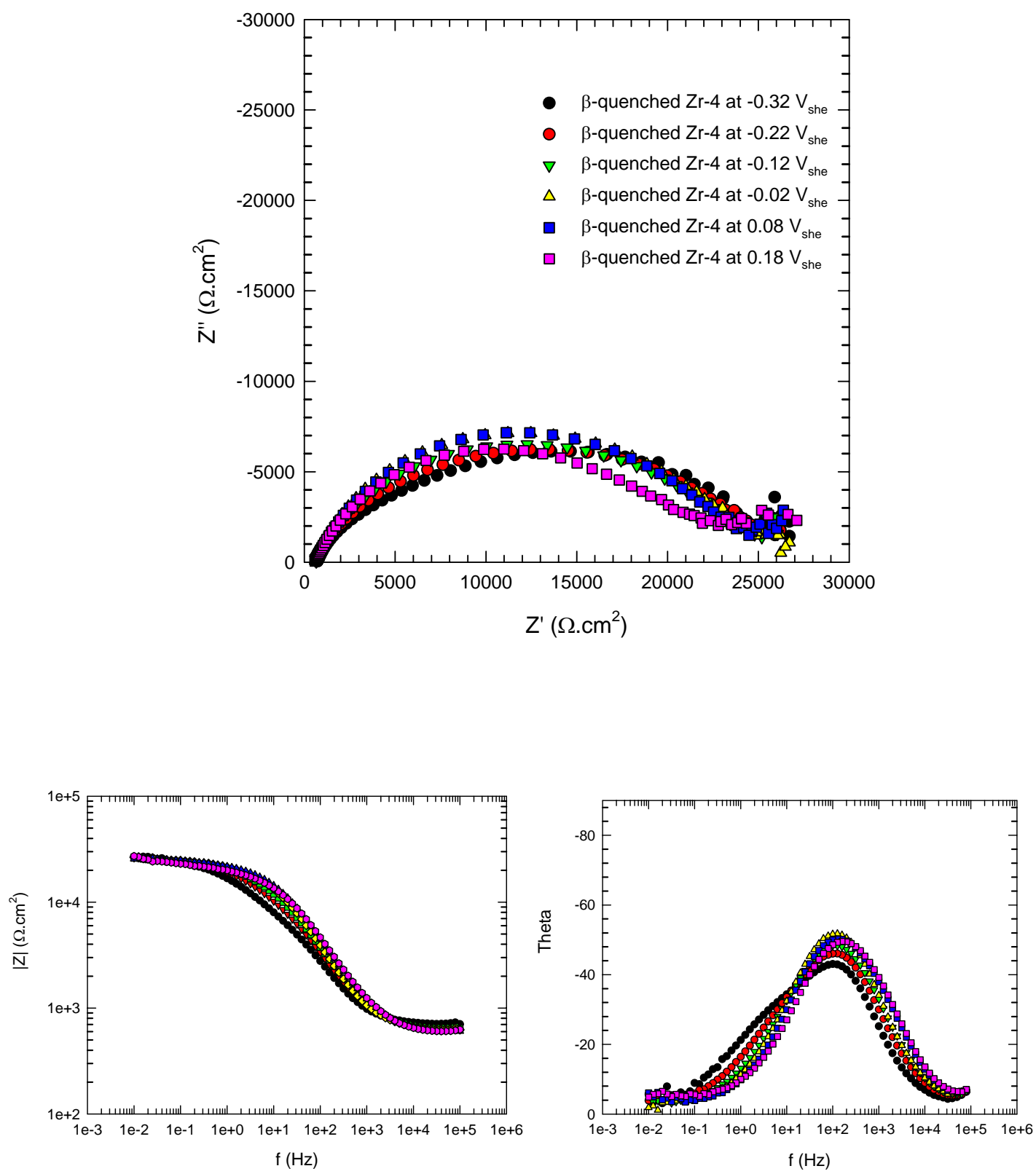

Figure 6-37: Impedance spectra for passive film on $\beta$-quenched Zircaloy-4 sample as a function of formation potential in $0.1 \mathrm{M} \mathrm{B}(\mathrm{OH})_{3}+0.001 \mathrm{M} \mathrm{LiOH}$ solutions with $45 \mathrm{~cm}^{3}(\mathrm{STP}) / \mathrm{kg}\left(\mathrm{H}_{2} \mathrm{O}\right)$ hydrogen. The impedance was measured after holding the potential at each potential step 24 hours for film growth. The potentials were changed in negative-to-the positive direction. $\mathrm{T}=250^{\circ} \mathrm{C}$ and $\mathrm{P}=62$ bar. 

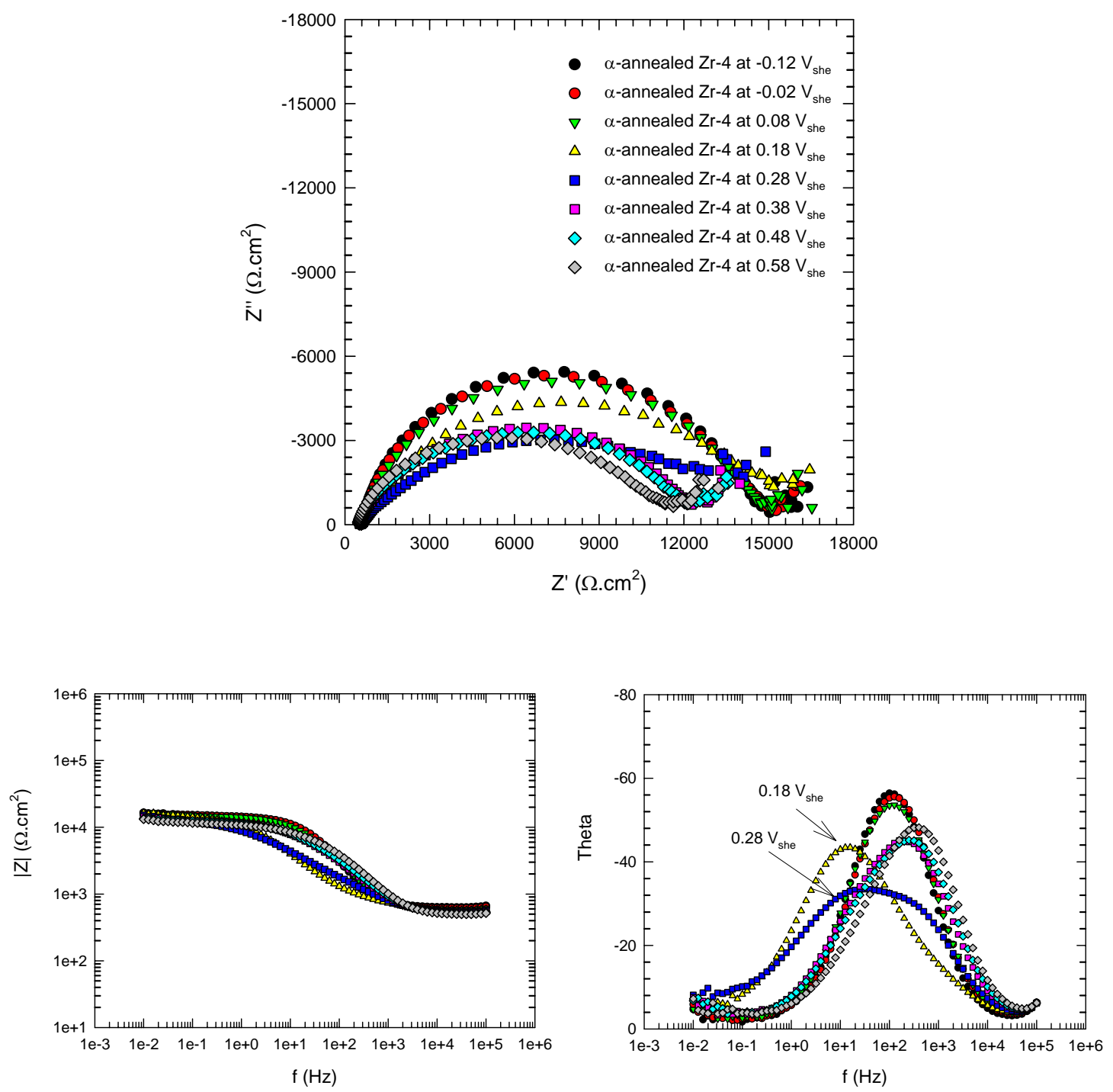

Figure 6-38: Impedance spectra for the passive films formed on the $\alpha$-annealed Zircaloy-4 sample as a function of formation potential in $0.1 \mathrm{M} \mathrm{B}(\mathrm{OH})_{3}+0.001 \mathrm{M}$ $\mathrm{LiOH}$ solutions with $45 \mathrm{~cm}^{3}(\mathrm{STP}) / \mathrm{kg}\left(\mathrm{H}_{2} \mathrm{O}\right)$ hydrogen. The impedance was measured after holding the potential at each potential step 24 hours for film growth. The potentials were changed from negative-to-the positive direction. $\mathrm{T}=$ $250^{\circ} \mathrm{C}$ and $\mathrm{P}=62$ bar. 

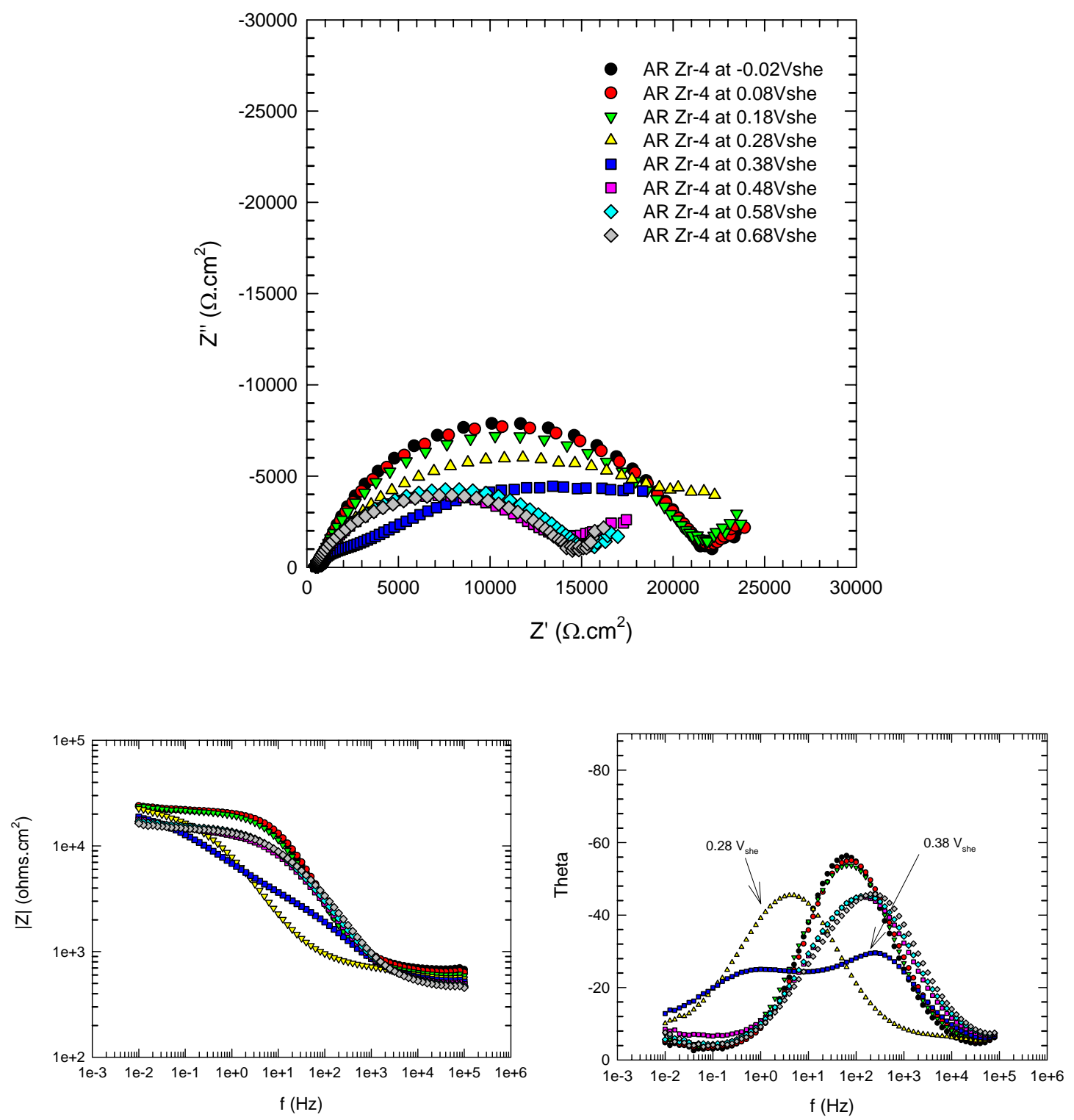

Figure 6-39: Impedance spectra for passive films on the as-received Zircaloy-4 sample as a function of formation potential in $0.1 \mathrm{M} \mathrm{B}(\mathrm{OH})_{3}+$ $0.001 \mathrm{M} \mathrm{LiOH}$ solutions with $45 \mathrm{~cm}^{3}(\mathrm{STP}) / \mathrm{kg}\left(\mathrm{H}_{2} \mathrm{O}\right)$ hydrogen. The impedance was measured after holding potential at each potential step 24 hours for film growth. The potentials were changed from negative to positive direction. $\mathrm{T}=$ $250^{\circ} \mathrm{C}$ and $\mathrm{P}=62$ bar. 


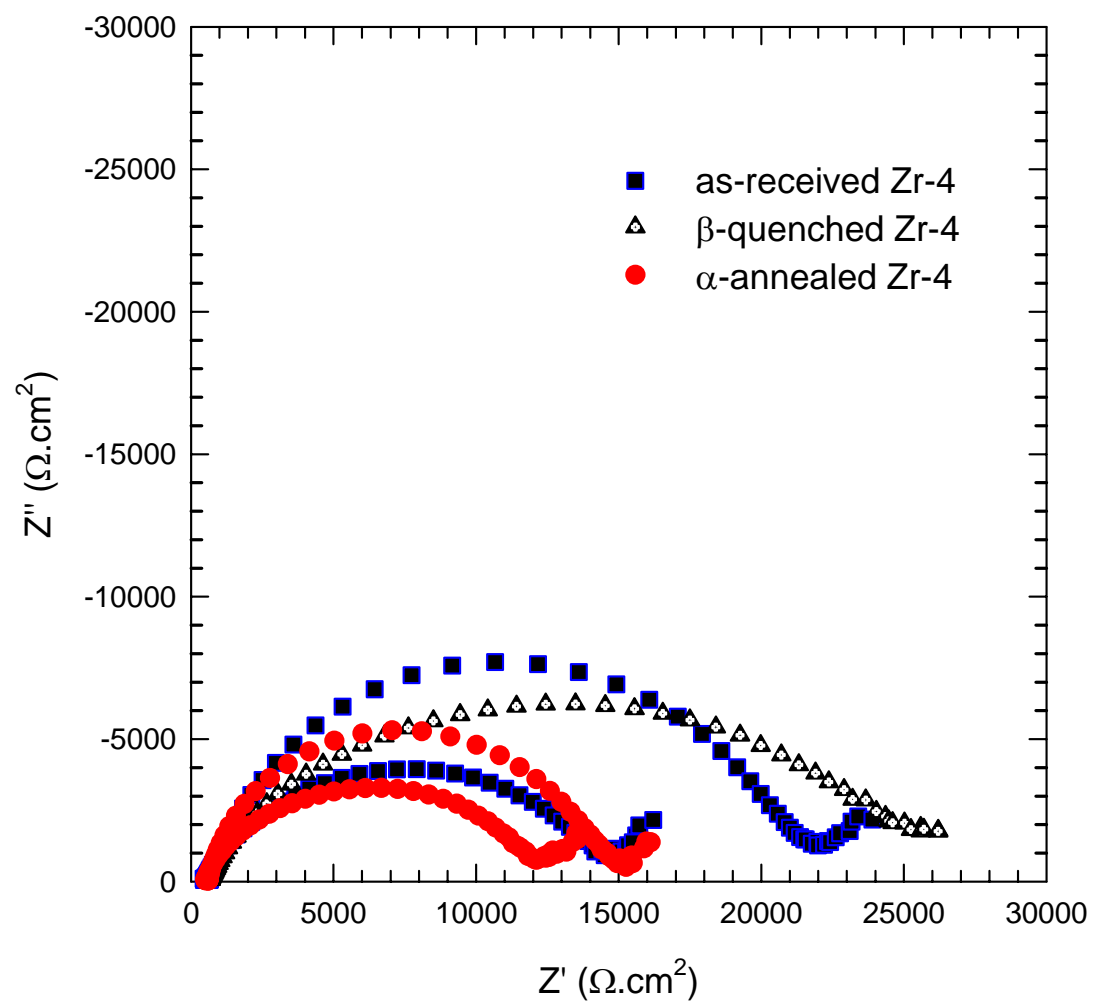

Figure 6-40: Comparison of the impedance spectra for passive films formed on the as-received, $\beta$-quenched and $\alpha$-annealed zircaloy- 4 samples by neglecting the transient parts in the $0.1 \mathrm{M} \mathrm{B}(\mathrm{OH})_{3}+0.001 \mathrm{M} \mathrm{LiOH}$ solutions with $45 \mathrm{~cm}^{3}(\mathrm{STP}) / \mathrm{kg}\left(\mathrm{H}_{2} \mathrm{O}\right)$ hydrogen. $\mathrm{T}=250^{\circ} \mathrm{C}$ and $\mathrm{P}=62$ bar.

In this work, after holding the specimen at the desired potential for 24 hours, in order for the film to reach steady state, the capacitance was recorded while simultaneously sweeping the voltage in the negative direction from the formation potential at a sweep rate of $100 \mathrm{mV} / \mathrm{s}$. This sweep rate is considered to be sufficiently high that the defect structure is "frozen-in" and hence that $N_{D}$ in Eq. (6-17) appears as a constant. Mott-Schottky plots are presented in Fig. 6-41, Fig. 6-42 and Fig. 6-43 for the passive films formed on the $\beta$-quenched, $\alpha$-annealed and as-received Zircaloy- 4 samples at different formation potentials in the hydrogenated borate buffer solutions at $250^{\circ} \mathrm{C}$ and 62 bar condition. From the positive slopes presented, it can be concluded that the passive film formed on the $\beta$-quenched zircaloy- 4 sample in the high temperature hydrogenated borate buffer solutions exhibits n-type electronic character. The value of $\mathrm{C}^{-2}$ increases with the potential except at $-0.02 \mathrm{~V}_{\text {she, }}$ where it has a lower film thickness comparing to its neighbors. The slopes for the as-received and $\alpha$-annealed Zircaloy- 4 samples are linearly positive for the passive films formed in the potentials lower than $0.28 \mathrm{~V}_{\text {she }}$ for the as-received Zircaloy- 4 and $0.18 \mathrm{~V}_{\text {she }}$ for the $\alpha$-annealed Zircaloy-4. The slope of MottSchottky plot is totally negative for the passive film formed at potential $0.28 \mathrm{~V}_{\text {she }}$ for the as-received Zircaloy-4 and $0.18 \mathrm{~V}_{\text {she }}$ for the $\alpha$-annealed Zircaloy-4. For the film formed at potentials higher than $0.28 \mathrm{~V}_{\text {she }}$ for the as-received Zircaloy- 4 and $0.18 \mathrm{~V}_{\text {she }}$ for the $\alpha$ annealed Zircaloy-4, the Mott-Schottky indicate a transient from positive to negative slope. The transient in the slope is believed caused by the second phase particles in the 
Zircaloy-4 samples. The positive slopes in the Mott-Schottky plots indicate that the dominant defects in the oxide film over this voltage range are mainly hydrogen vacancies and/or zirconium interstitials.

The donor densities $\mathrm{N}_{\mathrm{D}}$ at different formation potentials have been calculated from the slopes of Mott-Schottky plots for passive films formed on the $\beta$-quenched, asreceived, and $\alpha$-annealed zircaloy- 4 samples at different formation potentials as shown in Fig. 6-44. The donor density is highest in the $\beta$-quenched sample while lowest in the asreceived sample, which is in the same sequence as that for the density of second phase density in the samples. The data shown that the donor concentration in the vicinity of the metal/film interface decreases with increasing formation potential, in agreement with the prediction of the point defect model. The donor concentration, $\mathrm{N}_{\mathrm{D}}$, is in the range of $10^{17} \sim 10^{18} \mathrm{~cm}^{-3}$, which consistent with the $\mathrm{N}_{\mathrm{D}}<10^{19} \mathrm{~cm}^{-3}$ reported by Meisterjahn [15] for the zirconium passive film formed at room temperature. The much lower donor concentration found here demonstrates that the film is only lowly-doped with electron donors. However, the low doping level probably reflects a composite value for a highly doped, but thin, barrier layer and a thick stoichiometric outer layer.

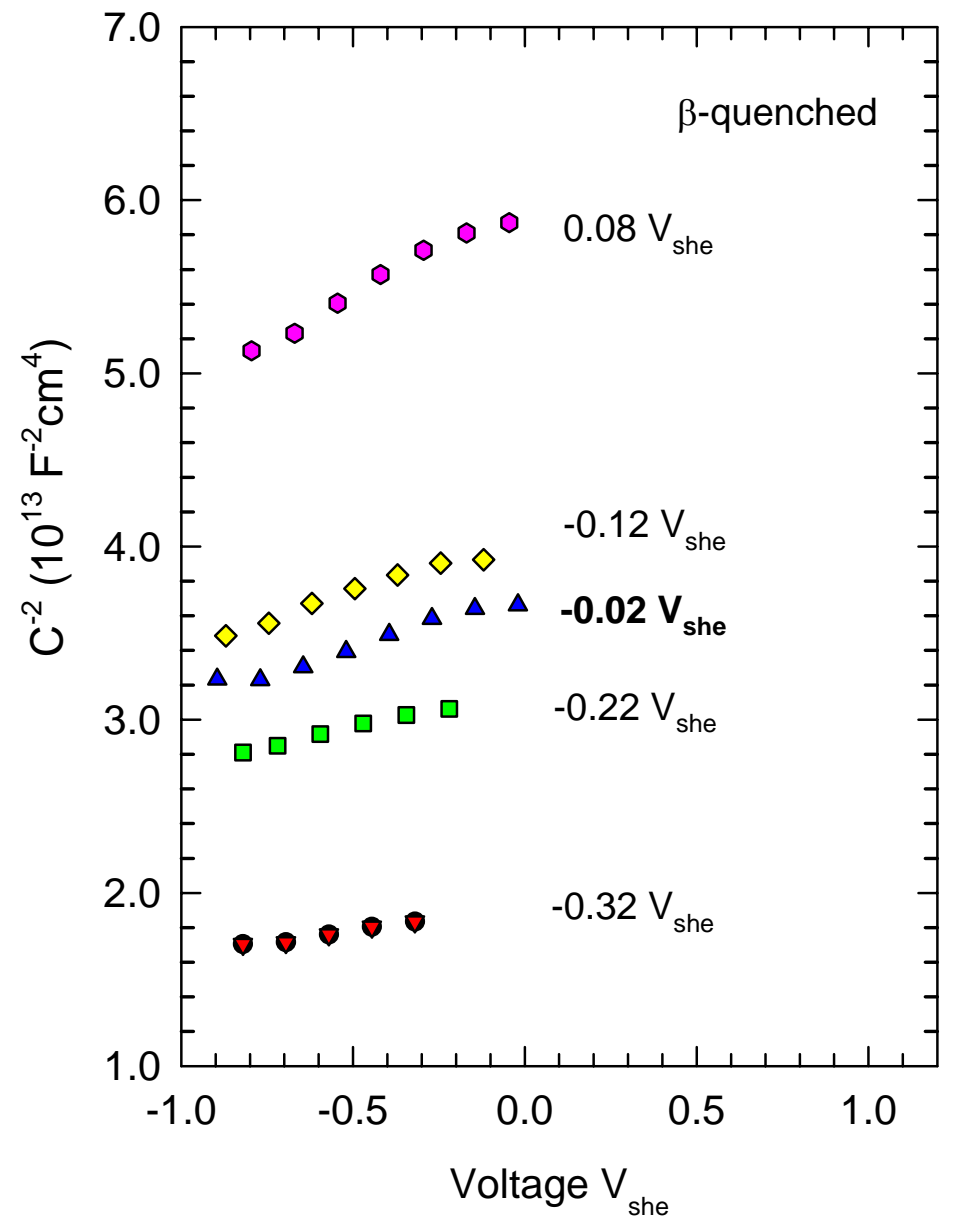

Figure 6-41: Mott-Schottky plots for the passive film formed on the $\beta$ quenched Zircaloy-4 sample at the indicated potentials after stabilization for 
24 hours at each point in $0.1 \mathrm{M} \mathrm{B}(\mathrm{OH})_{3}+0.001 \mathrm{M} \mathrm{LiOH}$ solutions with 45 $\mathrm{cm}^{3}(\mathrm{STP}) / \mathrm{kg}\left(\mathrm{H}_{2} \mathrm{O}\right)$ hydrogen. Sinusoidal excitation frequency $=5 \mathrm{kHz}$. Voltage sweep rate in the negative direction from the formation voltage $=100$ $\mathrm{mV} / \mathrm{s} . \mathrm{T}=250^{\circ} \mathrm{C}$ and $\mathrm{P}=62$ bar.

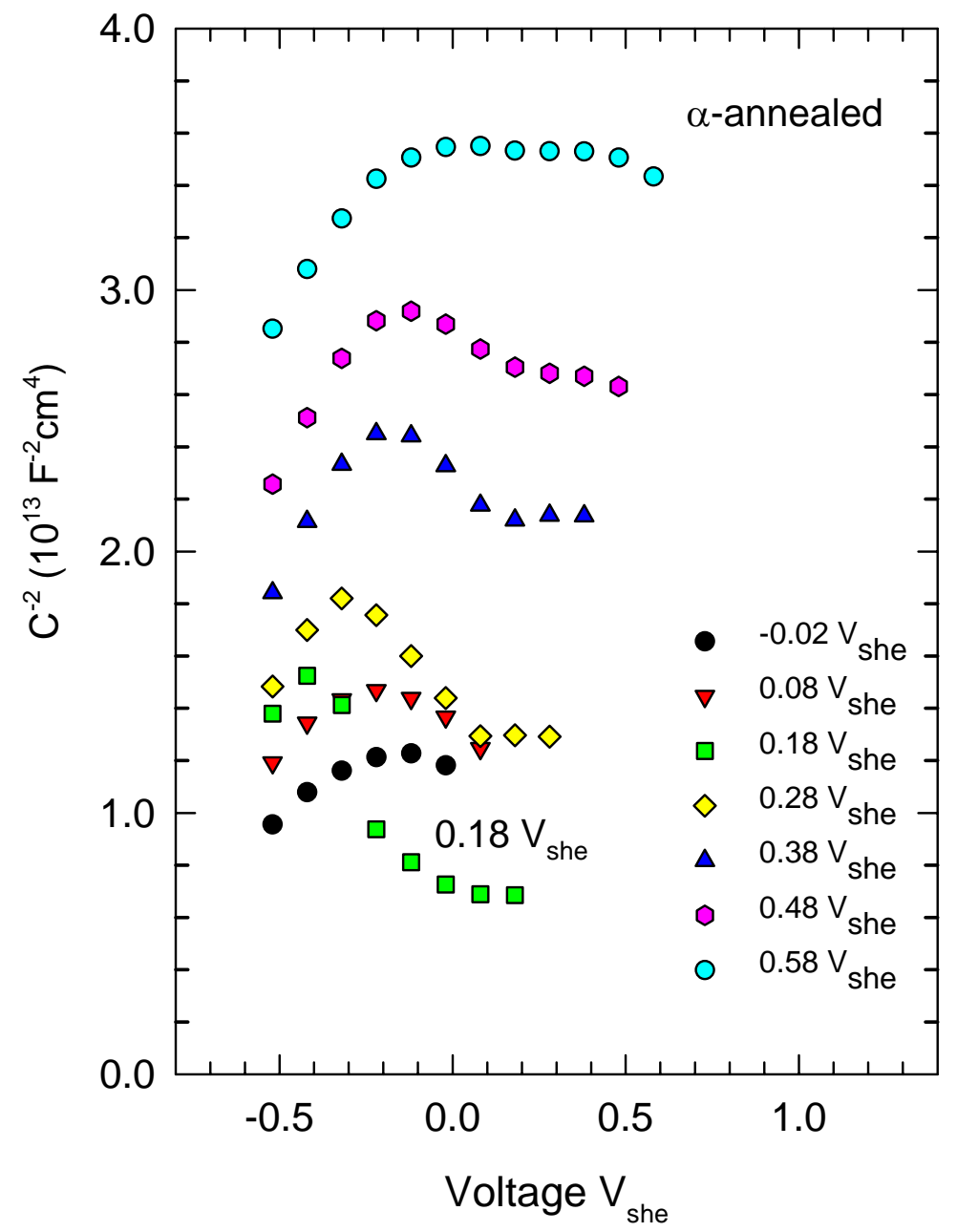

Figure 6-42. Mott-Schottky plots for the passive film formed on the $\alpha$ annealed zircaloy-4 sample at the indicated potentials after stabilization for 24 hours at each point in $0.1 \mathrm{M} \mathrm{B}(\mathrm{OH})_{3}+0.001 \mathrm{M} \mathrm{LiOH}$ solutions with 45 $\mathrm{cm}^{3}(\mathrm{STP}) / \mathrm{kg}\left(\mathrm{H}_{2} \mathrm{O}\right)$ hydrogen. Sinusoidal excitation frequency $=5 \mathrm{kHz}$. Voltage sweep rate in the negative direction from the formation voltage $=25$ $\mathrm{mV} / \mathrm{s} . \mathrm{T}=250^{\circ} \mathrm{C}$ and $\mathrm{P}=62$ bar. 


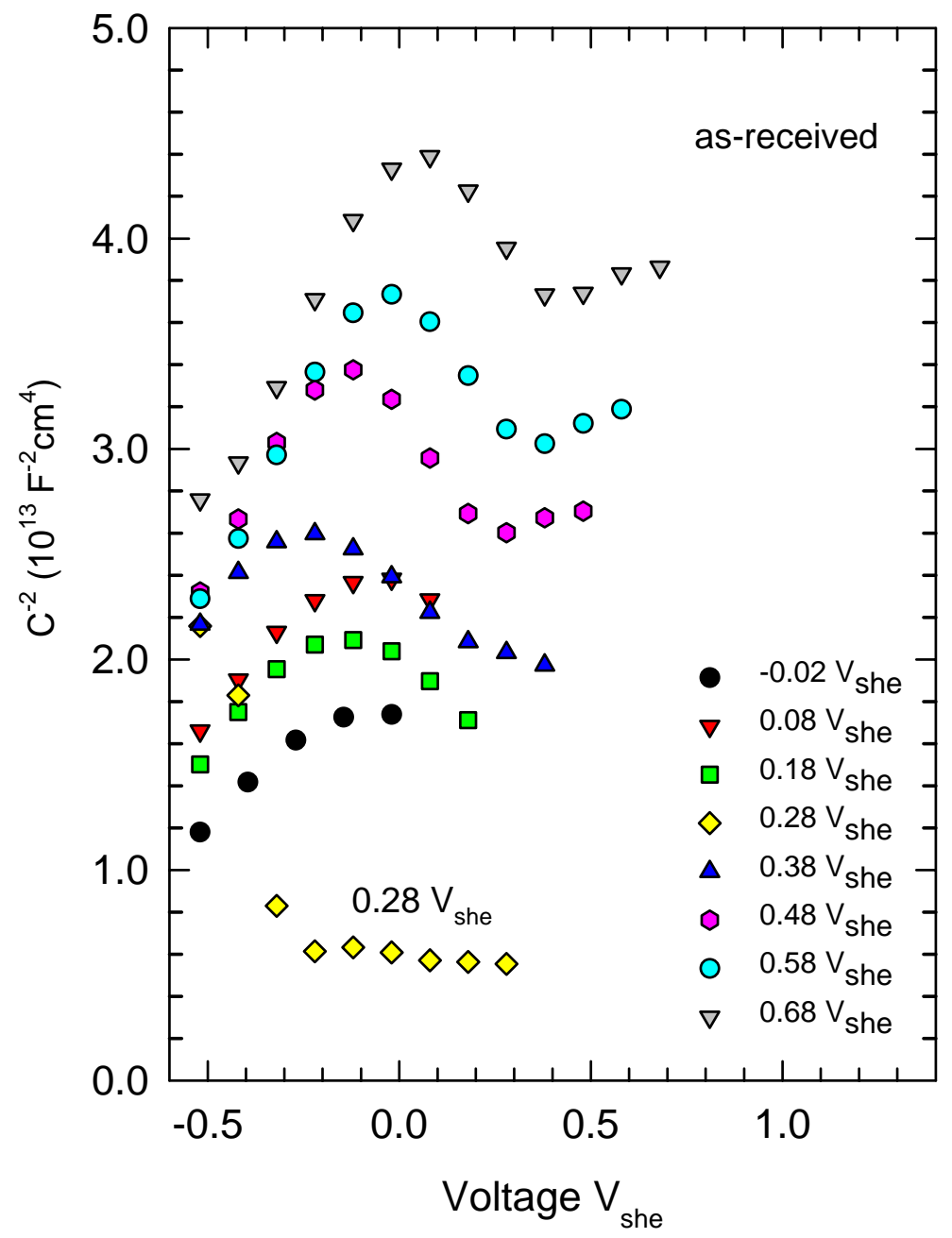

Figure 6-43. Mott-Schottky plots for the passive film formed on the asreceived $\mathrm{Zr}-4$ sample at the indicated potentials after stabilization for 24 hours at each point in $0.1 \mathrm{M} \mathrm{B}(\mathrm{OH})_{3}+0.001 \mathrm{M} \mathrm{LiOH}$ solutions with 45 $\mathrm{cm}^{3}(\mathrm{STP}) / \mathrm{kg}\left(\mathrm{H}_{2} \mathrm{O}\right)$ hydrogen. Sinusoidal excitation frequency $=5 \mathrm{kHz}$. Voltage sweep rate in the negative direction from the formation voltage $=25$ $\mathrm{mV} / \mathrm{s}$. $\mathrm{T}=250^{\circ} \mathrm{C}$ and $\mathrm{P}=62$ bar. 


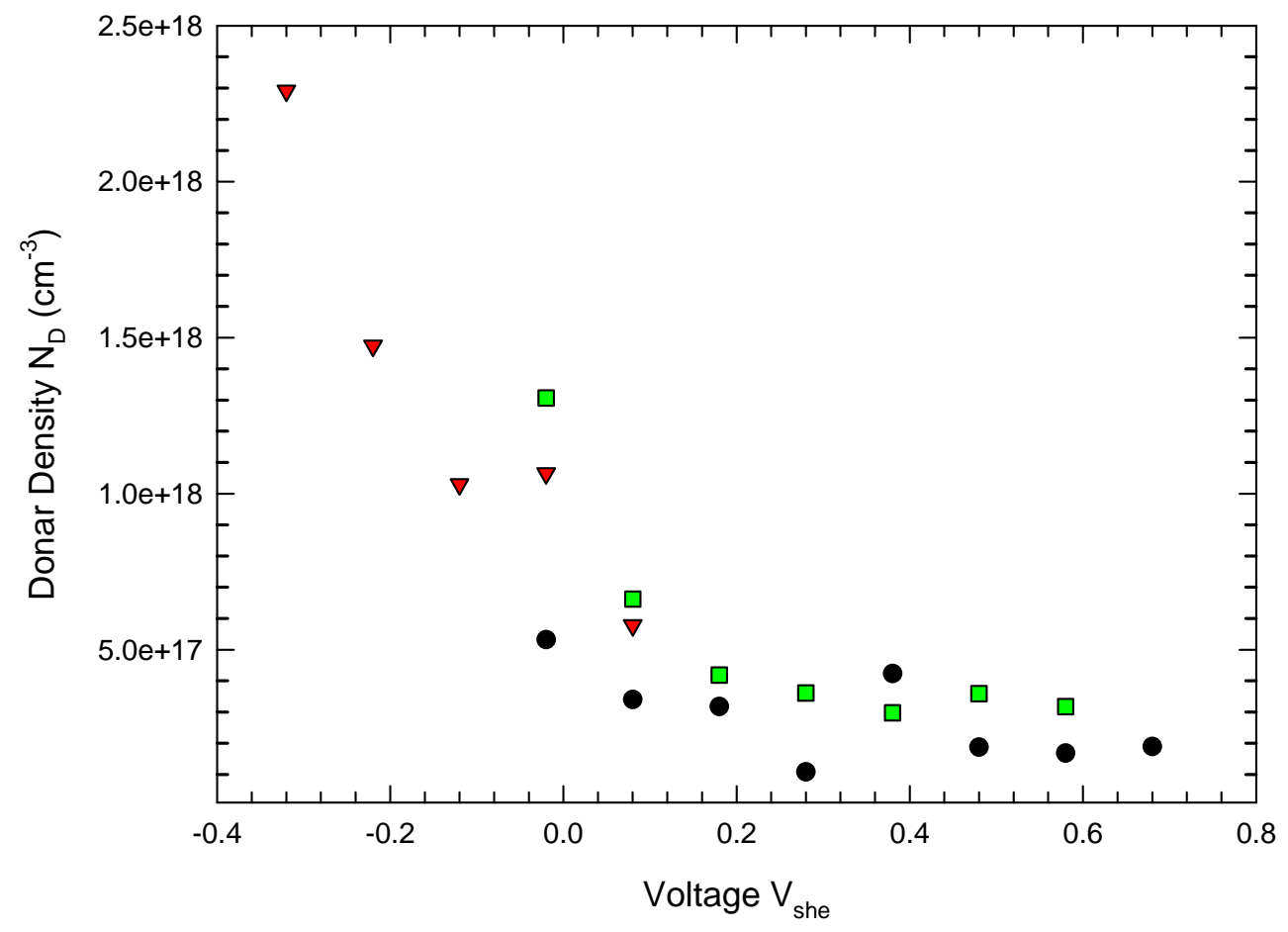

Figure 6-44. Donor density $\mathrm{N}_{\mathrm{D}}$ of the passive film formed on the as-received, $\beta$-quenched and $\alpha$-annealed Zircaloy-4 samples at different formation potential in $0.1 \mathrm{M} \mathrm{B}(\mathrm{OH})_{3}+0.001 \mathrm{M} \mathrm{LiOH}$ solutions with 45 $\mathrm{cm}^{3}(\mathrm{STP}) / \mathrm{kg}\left(\mathrm{H}_{2} \mathrm{O}\right)$ hydrogen. $\mathrm{T}=250^{\circ} \mathrm{C}$ and $\mathrm{P}=62$ bar.

\subsubsection{Summary and Conclusions}

In-situ studies have been carried out by using the controlled hydrodynamic apparatus to examine the effects of second phase particles on the electrochemistry of zirconium passive films by using different heat treated Zircaloy-4 samples, i.e., as received, $\beta$-quenched and $\alpha$-annealed samples. Heat treatment is used to control the size and density of the second phase particles in the Zircaloy-4 samples and optical microscopy and scanning electron microscopy (SEM) are used to characterize the morphological microstructure of heat treated zircaloy-4 samples and determine the size and density of second phase particles. The sizes of these second phase particles from small to large are in the sequence of $\beta$-quenched, $\alpha$-annealed and as-received and they are comparable to the thicknesses of the passive films. This similarity in dimension means that the corrosion process could be affected by these SPPs. $\beta$-quenched Zr-4 sample has the highest particle density while the as-received sample has the largest particle size. The second-phase particles in the $\alpha$-annealed zircaloy- 4 sample are mainly located in the grain boundary while randomly located in the zirconium matrix in the as received $\mathrm{Zr}-4$ sample. The appearance of a small spike in the anodic polarization curve is found to be related to the size and density of the second phase particles. The passive film properties are changed around the potentials where spike located as confirmed by the 
performances of passive films when measuring the impedance and capacitance as a function of potential. Second phase particles split the passive region into two parts with different corrosion resistance except in the $\beta$-quenched as shown by impedance measurements. The $\beta$-quenched $\mathrm{Zr}-4$ sample has the highest corrosion resistance while the $\alpha$-annealed sample is the lowest. The electronic characters of the passive films formed on the as-received and $\alpha$-annealed zircaloy- 4 samples are much more complex as indicated by the slope changes from positive to negative in the Mott-Shcottky plots while positive linear slopes are found for the $\beta$-quenched zircaloy-4 sample. The donor concentrations are in the range of $10^{17} \sim 10^{18} \mathrm{~cm}^{-3}$, which decreases with increasing formation potential, demonstrating that the film is only lowly-doped with electron donors. However, the low doping level probably reflects a composite value for a highly doped, but thin, barrier layer and a thick, stoichiometric outer layer.

\subsection{Electrochemistry of Zircaloy-2 in Hydrogenated Environment}

In this section, an in-situ study has been carried out to study the electrochemistry of passive Zircaloy-2 using the controlled hydrodynamic apparatus described before. The electrolyte was $0.1 \mathrm{M} \mathrm{B}(\mathrm{OH})_{3}+0.001 \mathrm{M} \mathrm{LiOH}$ with $45 \mathrm{~cm}^{3}(\mathrm{STP}) / \mathrm{kg}\left(\mathrm{H}_{2} \mathrm{O}\right)$ hydrogen. All experiments reported here were carried out at $250^{\circ} \mathrm{C}$ and under a pressure of $62 \mathrm{bar}$ (900 psi) in order to maintain a single liquid phase in the system. The Zircaloy-2 samples, with an exposed area of $4.49 \mathrm{~cm}^{2}$, used in this study were supplied by Western Zirconium in Ogden, UT. The composition is determined by Electron Probe Micro Analysis (EPMA), as shown in Table 6-3. The specimens were first mechanically polished with 400, 800 and 1200 grit $\mathrm{SiC}$ paper, and, then, further polished with $3 \mu \mathrm{m}$ and $1 \mu \mathrm{m}$ diamond paste.

Table 6-3: Chemical specification for Zircaloy-2 samples determined by Electron Probe Micro Analysis (EPMA), in wt\%

\begin{tabular}{|c|c|c|c|c|}
\hline Material & Sn & Ni & Fe & Cr \\
\hline $\mathbf{Z r}-2$ & 1.41 & 0.06 & 0.15 & 0.09 \\
\hline
\end{tabular}

\subsubsection{Polarization Studies}

The polarization curves were recorded by starting the voltage sweep at a rate of 1 $\mathrm{mVs}^{-1}$ from a point $0.2 \mathrm{~V}$ more negative than the zero current potential. Figure 6-45 shows the polarization curve of the Zircaloy-2 samples. The passive region ranges from 0.40 to $0.57 \mathrm{~V}_{\text {she }}$ with a slight increase in slope in the range of $0.18-0.28 \mathrm{~V}_{\text {she }}$. Frequently, the passive range of alloys exhibit a passive region followed by an active region and, then, a new re-passivation at more positive potentials. In the polarization curve of Zircaloy-2 shown in Figure 6-45, the initial passivation followed by an active dissolution and a second passivation is not clearly depicted; probably because the current density scale is too large (6 orders of magnitude). This phenomena of passivation-depassivationpassivation is believed to be caused by de-alloying of some minor alloy elements and local changes of the alloy surface characteristics. Alloying elements are dissolved during the cathodic polarization part. The polarization curve was recorded at $0.2 \mathrm{~V}$ more negative than the zero current potential. AC impedance is a technique which is sensitive 
to surface changes and clearly reflects the two-passive-regions behavior of passive films of Zircaloy-2 as a function of formation potential, as shown in Figure 6-46. The plots shown on these figure were obtained after holding the potential at the desired value for 24 hours for film growth in the $0.1 \mathrm{M} \mathrm{B}(\mathrm{OH})_{3}+0.001 \mathrm{M} \mathrm{LiOH}$ with $45 \mathrm{~cm}^{3}(\mathrm{STP}) / \mathrm{kg}\left(\mathrm{H}_{2} \mathrm{O}\right)$ hydrogen solution .

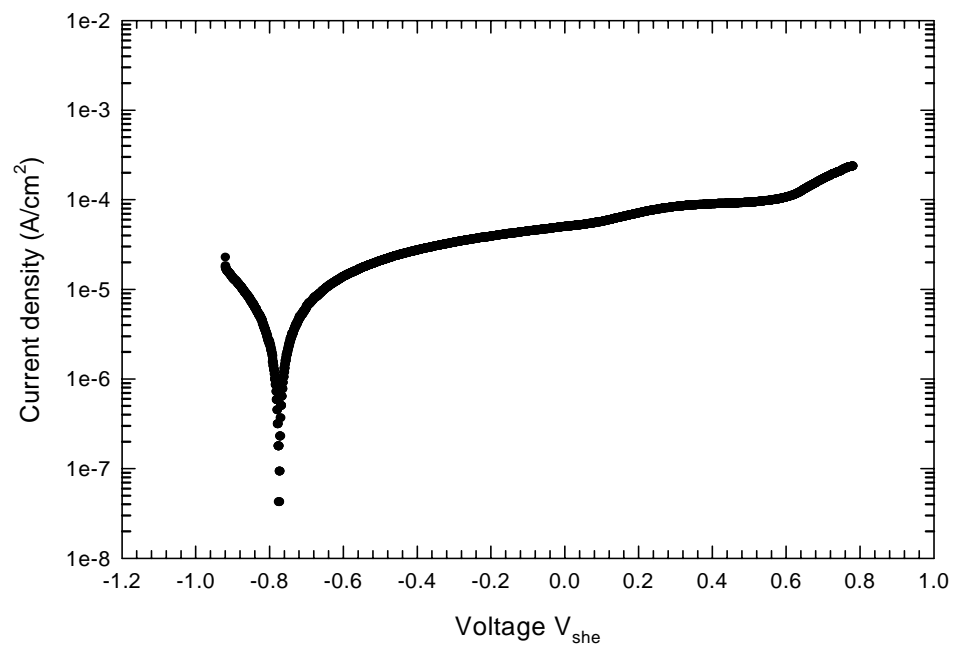

Figure 6-45: Polarization curve for $\mathrm{Zr}-2$ in a $0.1 \mathrm{M} \mathrm{B}(\mathrm{OH})_{3}+0.001 \mathrm{M} \mathrm{LiOH}$ with $45 \mathrm{~cm}^{3}(\mathrm{STP}) / \mathrm{kg}\left(\mathrm{H}_{2} \mathrm{O}\right)$ hydrogen solution. The hydrogen concentration was maintained in the reservoir by suitably controlling the total gas pressure in the system. Scan rate $=1.0 \mathrm{mV} / \mathrm{s}, \mathrm{T}=250^{\circ} \mathrm{C}$ and $\mathrm{P}=62 \mathrm{bar}$.

\subsubsection{Electrochemical Impedance Spectroscopy}

The corrosion resistance of Zircaloy-2 samples was determined by the electrochemical impedance measurements after the growth of passive film reached steady state by holding the formation potential constant at each point for 24 hours in the hydrogenated borate buffer solution at $250^{\circ} \mathrm{C}$ and 62 bar. Electrochemical impedance spectra (EIS) for passive films formed on the Zircaloy-2 surfaces were measured over a wide range of frequencies (typically $100 \mathrm{kHz}$ to $0.01 \mathrm{~Hz}$ ) as a function of the formation potential across the passive range. The measurements were carried out in the ascending potential direction. Since the stability of the electrochemical system during EIS measurement is critical to obtaining viable data, the quality of the EIS data was checked experimentally by stepping the frequencies from high-to-low and then immediately back from low-to-high, with the impedance being measured at each step to ascertain whether the same values were obtained at equivalent frequencies in the two directions. If the system is in the steady state, which means the thickness and current are independent of time, the impedance data should match in the two potential step directions. Impedance data measured in the two step directions were coincident for all the formation potentials. The impedance values of passive films formed on the $\mathrm{Zr}-2$ sample (Figure 6-46) as a function of formation potential clearly shows a change from the passive state to a more active state when the potential is shifted from $0.18 \mathrm{~V}_{\text {she }}$ to $0.28 \mathrm{~V}_{\text {she. Also shown in the }}$ same figure is the repassivation effect exhibited at $0.38 \mathrm{~V}_{\text {she. The impedances in the same }}$ passive region are independent of the formation potential, with a higher impedance value 
in the lower potentials and smaller impedances for higher potentials. This independence is also noticed in the Bode plot for formation potential in the same passive range. By measuring the impedance as a function of formation potential, it clearly shows there are two passive regions for Zircaloy-2, although it is difficult to divide the passive region in the polarization curve (Figure 6-45). Similar two-passive-region behavior has also been noticed in the $\alpha$-annealed and "as-received" Zircaloy-4 samples, as shown in Fig. 6-38 and 6-39. Figure 6-47 shows the comparison of the impedance spectra for passive films formed on the Zircaloy- 2 and Zircaloy-4 samples in the passive regions in the same solution. Zircaloy-2 shows lower corrosion resistances compared to Zircaloy-4.
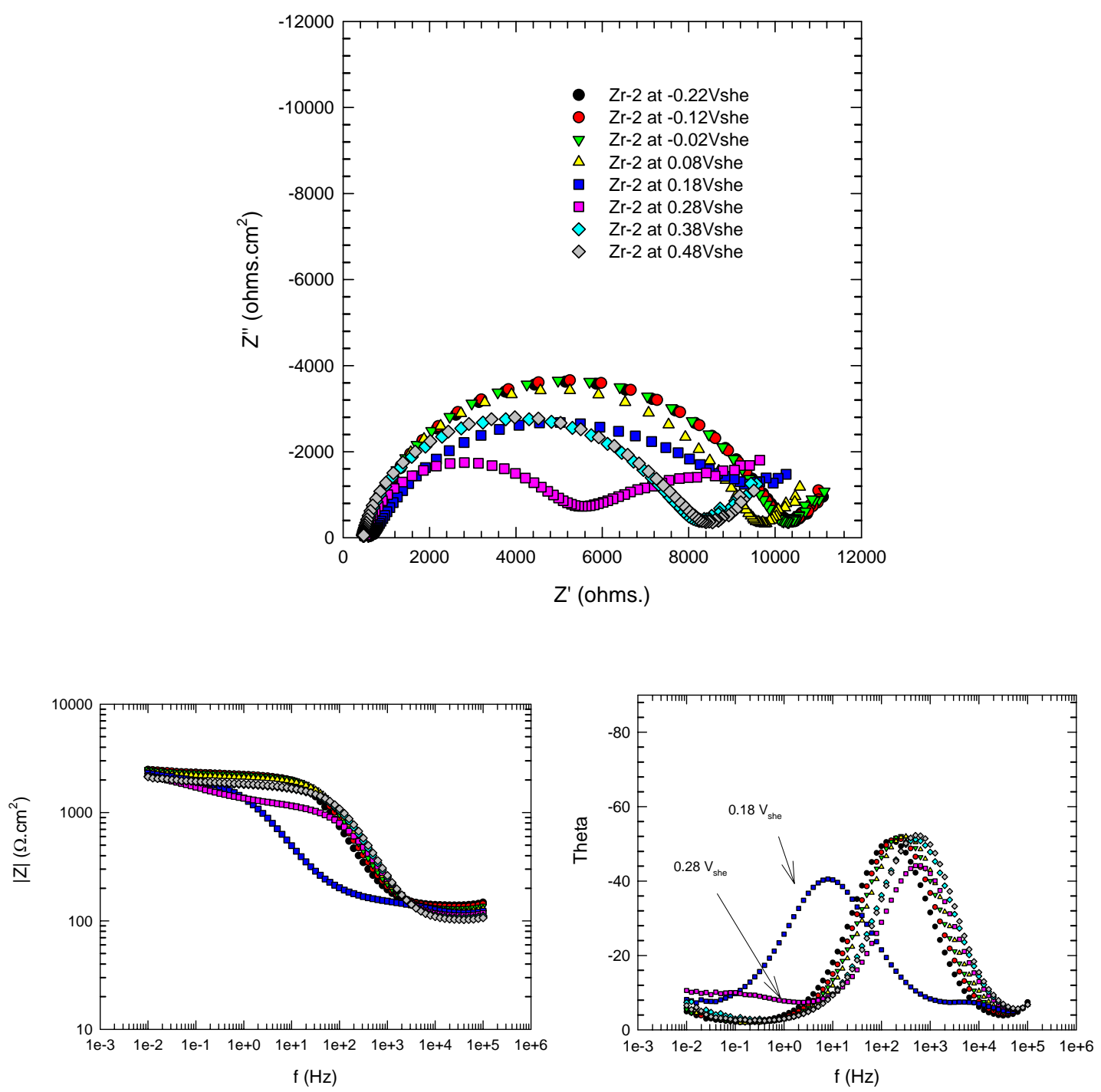

Figure 6-46: Impedance spectra for passive film on Zircaloy- 2 sample as a function of formation potential in the $0.1 \mathrm{M} \mathrm{B}(\mathrm{OH})_{3}+0.001 \mathrm{M} \mathrm{LiOH}$ solutions with $45 \mathrm{~cm}^{3}(\mathrm{STP}) / \mathrm{kg}\left(\mathrm{H}_{2} \mathrm{O}\right)$ hydrogen. The impedance was measured after holding the potential at each potential step 24 hours for film growth. The potentials were changed in negative-to-the positive direction. $\mathrm{T}=250^{\circ} \mathrm{C}$ and $\mathrm{P}=62$ bar. 


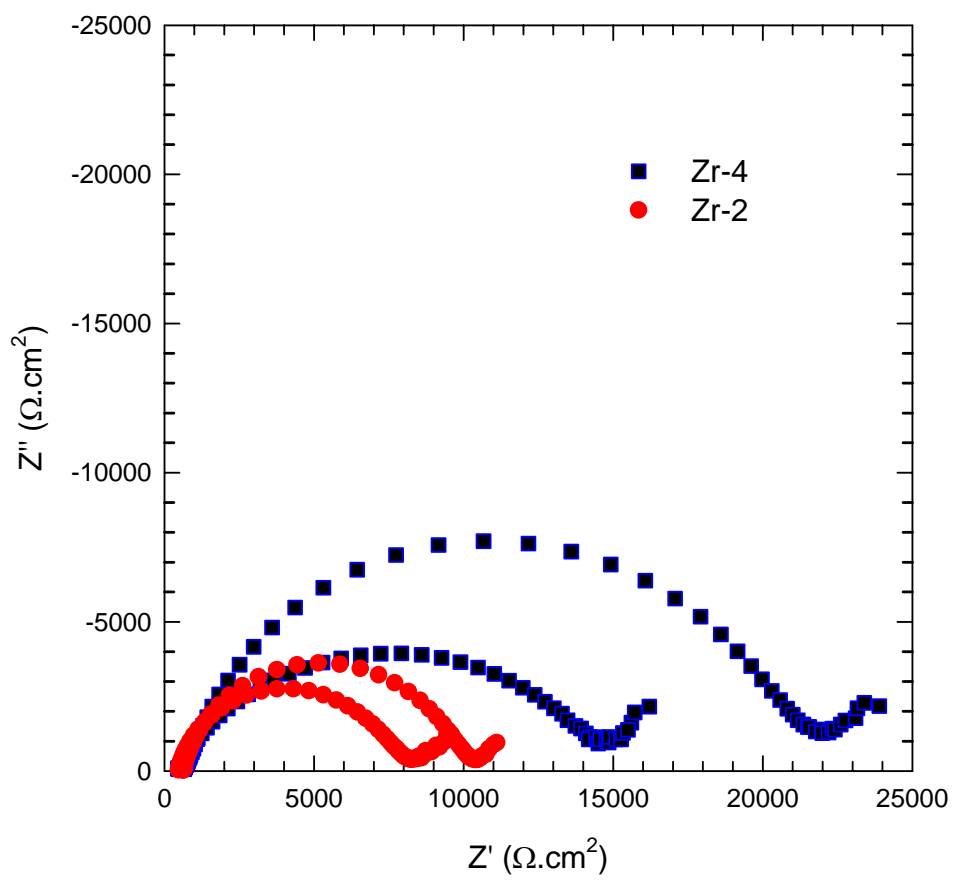

Figure 6-47: Comparison of the impedance spectra for passive films formed on the Zircaloy-2 and Zircaloy-4 samples in the two passive regions in the $0.1 \mathrm{M} \mathrm{B}(\mathrm{OH})_{3}+0.001 \mathrm{M} \mathrm{LiOH}$ solutions with $45 \mathrm{~cm}^{3}(\mathrm{STP}) / \mathrm{kg}\left(\mathrm{H}_{2} \mathrm{O}\right)$ hydrogen. $\mathrm{T}=250^{\circ} \mathrm{C}$ and $\mathrm{P}=62$ bar.

\subsubsection{Mott-Schottky Analysis}

An understanding of the crystallographic and electronic defect structures of the passive film on Zircaloy-2 is vital in exploring the underlying mechanisms of oxidation and hydriding of Zircaloy fuel cladding in reactor coolant environments. To identify the principal defect in the passive film, Mott-Schottky (M-S) analysis [2] as described in previous sections is being used. In M-S analysis, the capacitance of the passive film is measured at a suitably high frequency as a function of voltage. According to MottSchottky theory [20], the space charge capacitance $C_{s c}\left(\mathrm{~F} / \mathrm{cm}^{2}\right)$ of n-type and p-type semiconductor junctions are given by

and

$$
\frac{1}{C_{s c}^{2}}=\frac{2}{\varepsilon \varepsilon_{0} q N_{D} A^{2}}\left(E-E_{f b}-\frac{k T}{q}\right) \quad \text { n-type }
$$

$$
\frac{1}{C_{s c}^{2}}=\frac{-2}{\varepsilon \varepsilon_{0} q N_{A} A^{2}}\left(E-E_{f b}-\frac{k T}{q}\right) \quad \text { p-type }
$$

respectively. In these expressions, $N_{D} / N_{A}$ is the donor/acceptor concentration $\left(\mathrm{cm}^{-3}\right), \varepsilon_{0}$ is the vacuum permittivity $\left(8.85 \times 10^{-14} \mathrm{~F} / \mathrm{cm}\right), \varepsilon$ is the dielectric constant of the oxide, $V$ is the applied potential, $V_{f b}$ is the flat band potential, $q$ is the electron charge $\left(1.6 \times 10^{-19} \mathrm{C}\right)$, 
and $k T / q$ is about $45 \mathrm{mV}$ at $250^{\circ} \mathrm{C}$. In addition to confirming the electronic nature of the film, M-S analysis yields the dopant concentration (donor or acceptor) in the film as a function of voltage. The interfacial capacitance $C$ is obtained from $C=-1 / \omega Z$ " . Assuming that the capacitance of the Helmholtz layer can be neglected, the measured capacitance $C$ is equal to the 'space charge' capacitance, $\mathrm{C}_{\mathrm{sc}}$. Accordingly, a $C_{s c}{ }^{-2}$ versus $\mathrm{V}$ plot should be a straight line with a slope that is inversely proportional to the dopant concentration.

The outer layer of the passive film formed on zirconium is found to be much thicker than the barrier layer. The thin barrier layer is a defective phase with semiconductor properties that depend upon the type of defects present while the outer layer is relatively porous and has no appreciable semiconductor properties [6]. Therefore, the measured capacitance cannot be directly approximated as the space charge capacitance; instead, the observed capacitance $C$ can be understood by postulating that it reflects a series combination of a voltage-independent capacitance, $C_{o x}$, due to the outer layer insulting layer, and the space charge capacitance, $C_{b l}$, of the barrier layer, as discussed in the thickness calculation section of this paper.

$$
\frac{1}{C}=\frac{1}{C_{o x}}+\frac{1}{C_{b l}}
$$

It has been shown by de Gryse et al. [21] that the measured capacitance can be related to $C_{o x}$ and the applied voltage by

$$
\frac{1}{C^{2}}=\frac{1}{C_{o x}^{2}}+\frac{2}{\varepsilon \varepsilon_{0} q N_{D}}\left(V-V_{f b}-\frac{k T}{q}\right)
$$

where the notation is same as previously defined. As can be seen from this expression, the slope of the MS-plot is not influenced by the oxide capacitance $C_{o x}$ and can be used to derive reliable values for the donor density, $N_{D}$, from the slope of the measured MS-plot, although the intercept is shifted on the vertical and horizontal axes, thereby yielding a flat band potential that is too negative. Mott-Schottky analysis can be applied to a system of fixed (voltage-independent) total dopant concentration and, hence, $C$ versus $V$ must be measured under the conditions that do not result in a change in the dopant concentration profile of the film.

In this work, after holding the specimen at the desired potential for 24 hours, in order for the film to reach steady state, the capacitance was recorded while simultaneously sweeping the voltage in the negative direction from the formation potential at a sweep rate of $100 \mathrm{mV} / \mathrm{s}$. This sweep rate is considered to be sufficiently high that the defect structure is "frozen-in" and hence that $N_{D}$ in Eq. (6-17) appears as a constant. Mott-Schottky plots are presented in Fig. 6-48 for the passive films formed on the Zircaloy-2 samples at different formation potentials in the hydrogenated borate buffer solutions at $250^{\circ} \mathrm{C}$ and 62 bar condition. From the positive slopes presented, it can be concluded that the passive film formed on the zircaloy-2 sample in the high temperature hydrogenated borate buffer solutions exhibits n-type electronic character. The value of $\mathrm{C}^{-2}$ increases with the potential except at $0.18 \mathrm{~V}_{\text {she }}$ which happens to be in the depassivation region. The change in the slope of the Mott-Schottky plot is believed caused by the second phase particles in the Zircaloy-2 samples. The positive slopes in 
the Mott-Schottky plots indicate that the dominant defects in the oxide film over this voltage range are mainly hydrogen vacancies and/or zirconium interstitials.

The donor densities $\mathrm{N}_{\mathrm{D}}$ at different formation potentials have been calculated from the slopes of Mott-Schottky plots for passive films formed on Zircaloy-2 at different formation potentials as shown in Fig. 6-49. The data shown that the donor concentration in the vicinity of the metal/film interface decreases with increasing formation potential except at the potential of $0.18 \mathrm{~V}_{\text {she }}$, which attributes to the existing of second phase particles in Zircaloy-2, in agreement with the prediction of the point defect model. The donor concentration, $\mathrm{N}_{\mathrm{D}}$, is in the range of $10^{17} \sim 10^{18} \mathrm{~cm}^{-3}$, which consistent with the $\mathrm{N}_{\mathrm{D}}$ $<10^{19} \mathrm{~cm}^{-3}$ reported by Meisterjahn [15] for the zirconium passive film formed at room temperature. The much lower donor concentration found here demonstrates that the film is only lowly-doped with electron donors. However, the low doping level probably reflects a composite value for a highly doped, but thin, barrier layer and a thick stoichiometric outer layer.

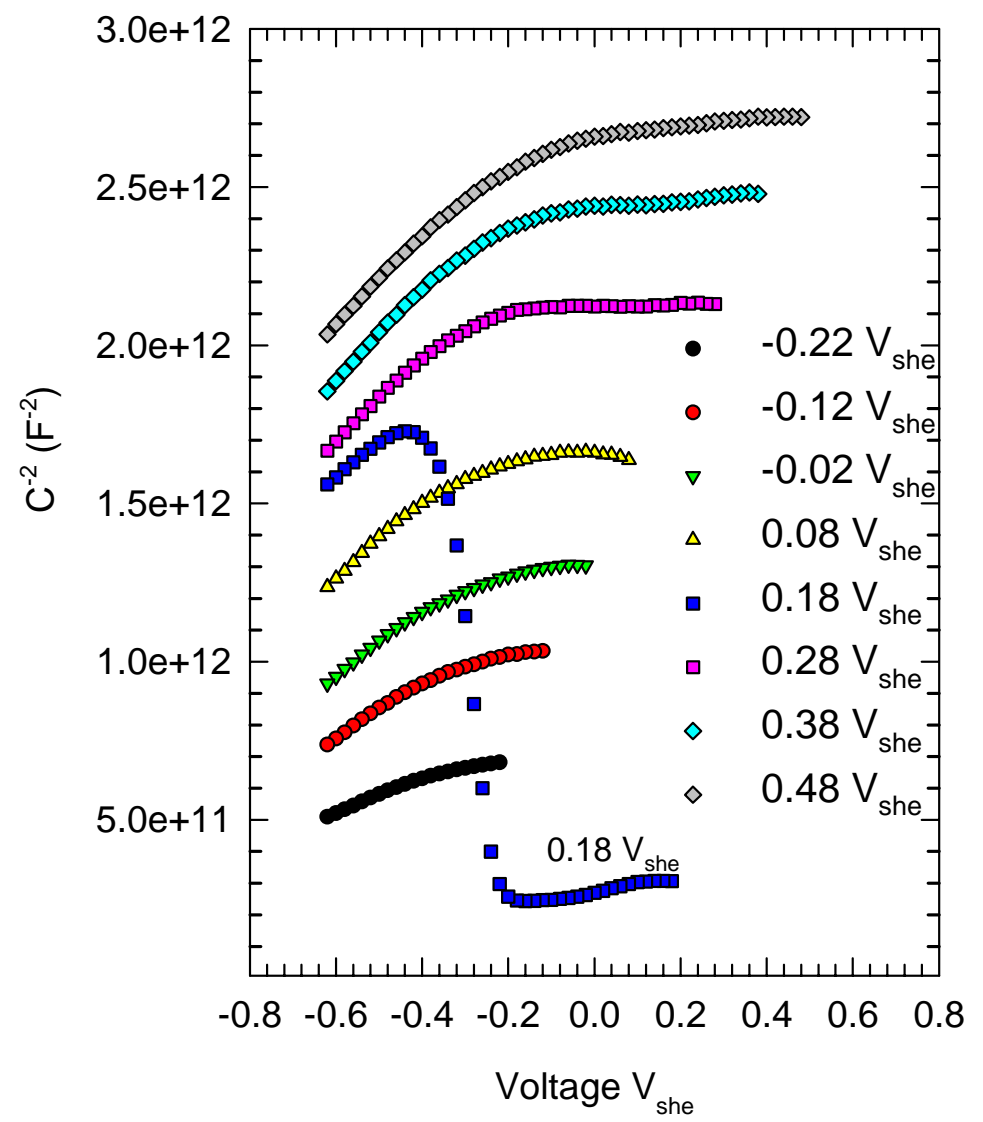

Figure 6-48. Mott-Schottky plots for the passive film formed on the Zircaloy2 sample at the indicated potentials after stabilization for 24 hours at each point in $0.1 \mathrm{M} \mathrm{B}(\mathrm{OH})_{3}+0.001 \mathrm{M} \mathrm{LiOH}$ solutions with $45 \mathrm{~cm}^{3}(\mathrm{STP}) / \mathrm{kg}\left(\mathrm{H}_{2} \mathrm{O}\right)$ hydrogen. Sinusoidal excitation frequency $=5 \mathrm{kHz}$. Voltage sweep rate in the negative direction from the formation voltage $=100 \mathrm{mV} / \mathrm{s} . \mathrm{T}=250^{\circ} \mathrm{C}$ and $\mathrm{P}=62$ bar. 


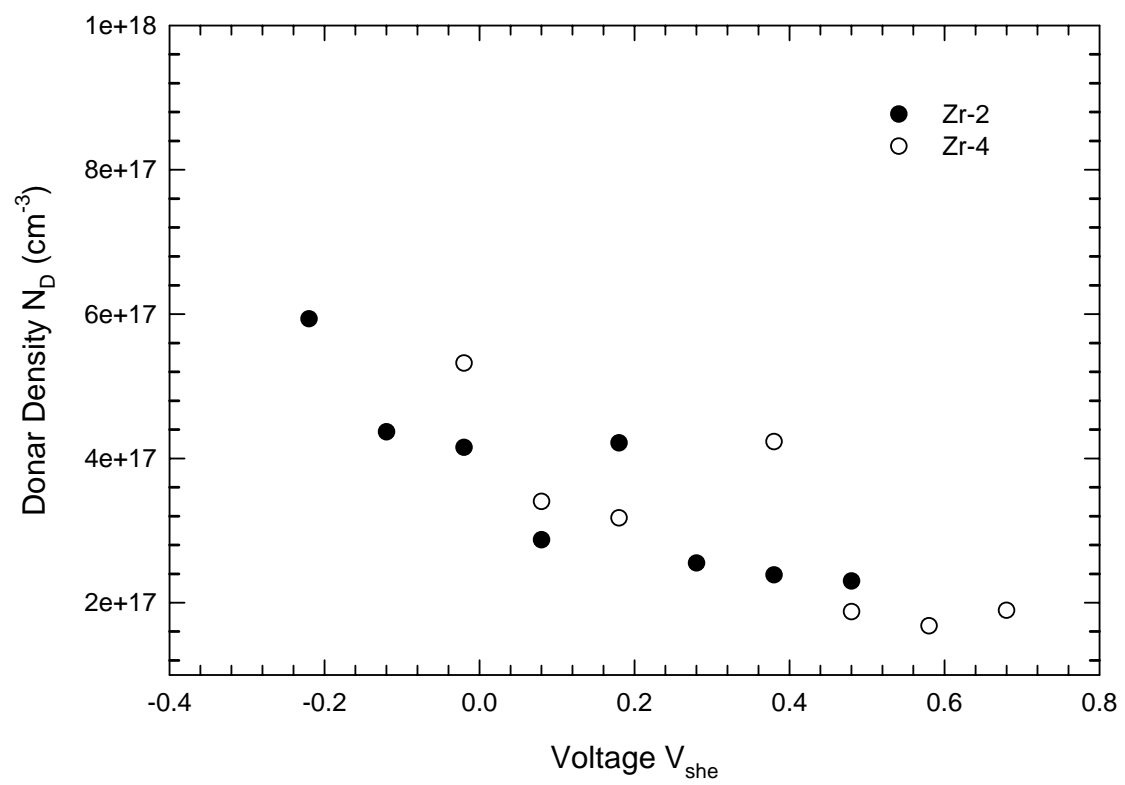

Figure 6-49. Donor density $\mathrm{N}_{\mathrm{D}}$ of the passive films formed on the Zircaloy-2 and Zircaloy-4 samples at different formation potential in $0.1 \mathrm{M} \mathrm{B}(\mathrm{OH})_{3}+$ $0.001 \mathrm{M} \mathrm{LiOH}$ solutions with $45 \mathrm{~cm}^{3}(\mathrm{STP}) / \mathrm{kg}\left(\mathrm{H}_{2} \mathrm{O}\right)$ hydrogen. $\mathrm{T}=250{ }^{\circ} \mathrm{C}$ and $\mathrm{P}=62$ bar.

\subsubsection{Summary and Conclusions}

In-situ studies have been carried out by using the controlled hydrodynamic apparatus to examine the electrochemistry of Zircaloy-2 samples in the high temperature, high pressure, hydrogenated borate buffer solution environment. Second phase particles split the passive region into two parts with different corrosion resistance as shown by impedance measurements as a function of formation potential. The impedance of Zircaloy-2 is lower than that of Zircaloy- 4 as expected in the hydrogenated environment due to the existence of nickel in the Zircaloy-2 sample. The passive films formed on the Zircaloy-2 samples are n-type in electronic character as indicated by the positive slopes of Mott-Shcottky plots and the dominant defects in the oxide film over this voltage range are mainly hydrogen vacancies and/or zirconium interstitials. The donor concentrations are in the range of $10^{17} \sim 10^{18} \mathrm{~cm}^{-3}$, which decreases with increasing formation potential, demonstrating that the film is only lowly-doped with electron donors. However, the low doping level probably reflects a composite value for a highly doped, but thin, barrier layer and a thick, stoichiometric outer layer.

\section{Issues/Concerns: None}

\section{Reference}

1. Macdonald, D.D., J Electrochem Soc, 139(12): (1992). p. 3434-3449.

2. Macdonald, D.D., Pure Appl Chem, 71(6): (1999). p. 951-978. 
3. Pensado-Rodriguez, O., J.R. Flores, M. Urquidi-Macdonald, and D.D. Macdonald, J Electrochem Soc, 146(4): (1999). p. 1326-1335.

4. Pensado-Rodriguez, O., M. Urquidi-Macdonald, and D.D. Macdonald, J Electrochem Soc, 146(4): (1999). p. 1318-1325.

5. $\quad$ Pensado-Rodriguez, O., M. Urquidi-Macdonald, and D.D. Macdonald, J Electrochem Soc, 148(10): (2001). p. B386-B399.

6. Park, P.Y., M.U. Macdonald, and D.D. Macdonald. Application of the PDM (Point Defect Model) to the Oxidation of Zircaloy Fuel Cladding in Water-Cooled Nuclear Reactors. in ICONE 12.(2004).

7. Urquidi-Macdonald, M. and D.D. Macdonald, to be published.

8. Yeh, T.K. and D.D. Macdonald, Nucl Sci Eng, 123(2): (1996). p. 295-304.

9. HKLTechnology, http://www.hkltechnology.com/data/0-zriconium-hydride.pdf.

10. Wang, Z.F., C.L. Briant, and K.S. Kumar, Corrosion, 54(7): (1998). p. 553-560.

11. IAEA, Waterside corrosion of zirconium alloys in nuclear power plants, in IAEATECDOC-684. (1998).

12. Blat, M. and D. Noel, in Zirconium in the nuclear industry : eleventh international symposium, G.P. Sabol and E.R. Bradley, Editors. Astm, West Conshohocken, PA, (1996), p. 319.

13. Garde, A.M., G.P. Smith, and R.C. Pirck, in Zirconium in the nuclear industry : eleventh international symposium, G.P. Sabol and E.R. Bradley, Editors. Astm, West Conshohocken, PA, (1996), p. 407.

14. Kido, T., in Sixth proceedings of the international symposium on environmental degradation of materials in nuclear power systems-water reactors, R.E. Gold and E.P. Simonen, Editors, (1993).

15. Zhang, L., D.D. Macdonald, E. Sikora, and J. Sikora, J Electrochem Soc, 145(3): (1998). p. 898-905.

16. Urquidi-Macdonald, M., S. Real, and D.D. Macdonald, J Electrochem Soc, 133(3): (1986). p. C132-C132.

17. Urquidi-Macdonald, M., S. Real, and D.D. Macdonald, J Electrochem Soc, 133(10): (1986). p. 2018-2024.

18. Macdonald, D.D., M. Urquidimacdonald, and S. Real, J Electrochem Soc, 134(8B): (1987). p. C419-C420.

19. Sikora, E. and D.D. Macdonald, Electrochim Acta, 48(1): (2002). p. 69-77.

20. Sze, S., The Physics of Semiconductor Devices (2nd Edit.). Wiley \& Sons, N.Y., (1981).

21. Gryse, R.D., W.P. Gomes, F. Cardon, and V. Vennik, J. Electrochem. Soc., 122: (1975). p. 711.

22. Meisterjahn, P., H.W. Hoppe, and J.W. Schultze, J Electroanal Chem, 217(1): (1987). p. 159-185.

23. Macdonald, D.D., M. Al Rifaie, and G.R. Engelhardt, J Electrochem Soc, 148(9): (2001). p. B343-B347.

24. Erwin, K.T., O. Delaire, A.T. Motta, Y.S. Chu, D.C. Mancini, and R.C. Birtcher, J Nucl Mater, 294(3): (2001). p. 299-304.

25. Wikmark, G., P. Rudling, B. Lehtinen, B. Hutchinson, A. OScarsson, and E. Ahlberg, in Zirconium in the Nuclear Industry : Eleventh International Symposium, G.P. Sabol and E.R. Bradley, Editors. ASTM, West Conshohocken, PA, (1996), p. 55-73.

26. Gebhardt, O., A. Hermann, G. Bart, H. Blank, F. Garzarolli, and E.L.F. Ray, in Zirconium in the Nuclear Industry : Eleventh International Symposium, G.P. 
Sabol and E.R. Bradley, Editors. ASTM, West Conshohocken, PA, (1996), p. 218-241.

27. Goll, W. and I. Ray, in Zirconium in the Nuclear Industry: Thirteenth International Symposium, G.D. Moan and P. Rudling, Editors. ASTM, West Conshohocken, Pa., (2002), p. 80.

28. Charquet, D., J Nucl Mater, 211(3): (1994). p. 259-261.

29. Miyake, C., A. Nakatani, and T. Isobe, J Nucl Mater, 217(1-2): (1994). p. 172177.

30. Bangaru, N.R.V., J Nucl Mater, 131: (1985). p. 280-290.

31. Jeong, Y.H., K.S. Rheem, C.S. Choi, and Y.S. Kim, J Nucl Sci Technol, 30(2): (1993). p. 154-163.

32. Huang, K.-Y. and C.-H. Tsai, J Nucl Mater, 136: (1985). p. 16-29.

33. Foster, J.P., J. Dougherty, M.G. Burke, J.F. Bates, and S. Worcester, J Nucl Mater, 173(2): (1990). p. 164-178.

34. Gros, J.P. and J.F. Wadier, J Nucl Mater, 172(1): (1990). p. 85-96.

35. Mills, K. and ASM Handbook Committee., Metallography and microstructures. Metals handbook. Vol. 9th ed., v. 9. American Society for Metals. Metals Park, Ohio, (1985).

\section{Appendix:}

Table 6-3. Calculated potentials for the $\mathrm{Ag} / \mathrm{AgCl}$ (sat. $\mathrm{KCl}$ ) reference electrode vs. the Standard Hydrogen Electrode (SHE) at elevated temperature.

\begin{tabular}{|cc|}
\hline T $(\mathbf{O C})$ & E (volts vs. SHE) \\
\hline 0 & 0.222 \\
20 & 0.202 \\
50 & 0.170 \\
100 & 0.112 \\
150 & 0.048 \\
200 & -0.022 \\
210 & -0.037 \\
220 & -0.053 \\
230 & -0.068 \\
240 & -0.084 \\
250 & -0.101 \\
260 & -0.118 \\
270 & -0.135 \\
280 & -0.153 \\
290 & -0.171 \\
300 & -0.190 \\
310 & -0.209 \\
320 & -0.229 \\
330 & -0.249 \\
340 & -0.270 \\
350 & -0.291 \\
\hline
\end{tabular}




\section{Task 7. Model Integration.}

Objectives: In this task, we will integrate all of the models (PDM, radiolysis model for the coolant, BCM, MPM, etc) to produce a comprehensive algorithm for the oxidation of zirconium and of Zircaloy fuel sheathing under simulated reactor operating conditions. The models and algorithm will then be subjected to extensive sensitivity analysis, in order to rank the model parameters that have the greatest impact on the predictions of the code. Emphasis will be placed on determining, where possible, accurate values for these parameters, as proposed in Task 6 .

Task Status: The Point Defect Model (PDM) has now been integrated with the Boiling Crevice Model (BCM) for predicting concentration factors in CRUD on fuel surfaces, with the Mixed Potential Model (MPM) for calculating the ECP and with the radiolysis models for estimating the concentrations of electroactive radiolysis products in the cores of the reactors. While the codes are maintained as separate entities, because they are used individually for a variety of other purposes, the out put of one is designed to be used as the input for the following code. According, the work scheduled for this task has been completed.

Issues/Concerns: None.

\section{Task 8. Benchmarking and Evaluation.}

Objectives: Benchmarking the model against Zircaloy oxidation data from both BWRs and PWRs is an essential part of this program. As a result of an extensive literature search and the experimental work that will be performed in this project, we believe that we have available essentially all of the oxide film growth rate data that will be required to guide the development of the model and to test its validity. However, the design and operational characteristics of BWRs and PWRs are sufficiently different that benchmarking will need to be reactor-type specific to ensure predictive accuracy.

Task Status: As described above, the models developed in this work account for many phenomena that are observed in the corrosion of Zircaloys in reactor coolant circuits. For example, the PDM predicts the formation of a zirconium hydride barrier layer in the case of a PWR coolant, but not in the case of a BWR coolant, and this prediction is borne out by observation. The model successfully account for the impedance characteristics of oxidized zirconium and Zircaloys as measured this work. We know of no other model that has successfully accounted for the impedance of zirconium in high temperature aqueous systems.

Issues/Concerns: None. 


\section{Task 9. Technology Transfer.}

Objectives: Transferring the technology to the US nuclear power industry will be the final task of this program. Technology transfer will involve instructing nuclear utility personnel on the fundamental basis and operation of the code. We will also provide ongoing technical support and upgrades, which are not costed in the present proposal.

Task Status: Nine presentations or publications have been presented to international meetings or submitted for publication in peer reviewed journals. We expect that an additional six papers will result from the work.

Issues/Concerns: None.

\section{STATUS SUMMARY OF NERI TASKS}

The project is on schedule as indicated by the following table.

\begin{tabular}{|l|l|l|}
\hline Milestone/Task Description & Planned Completion Date & $\begin{array}{l}\text { Actual Completion } \\
\text { Date }\end{array}$ \\
\hline 1. Definition of Environment & September 30 2003 & September 30 2003 \\
\hline 2. PDM Development & September 30 2004 & September 30 2004 \\
\hline 3. Incorporate PDM into MPM & September 30 2004 & September 30 2004 \\
\hline 4. Radiochemistry Modeling & September 30 2004 & September 30 2004 \\
\hline 5. Modeling of Growth Stresses & September 14 2006 & September 14 2006 \\
\hline 6. Experimental Measurements & September 14 2006 & September 14 2006 \\
\hline 7. Model Integration & September 30 2005 & September 30 2005 \\
\hline 8. Benchmarking and Evaluation. & September 30 2005 & September 30 2005 \\
\hline 9. Technology Transfer & September 14 2006 & September 14 2006 \\
\hline
\end{tabular}

Overall Status: The project has been successfully initiated and progress is being made at an acceptable rate. No "show-stopping" problems have been encountered in any of the tasks and none appear on the horizon. A no cost extension of the project to September 14 2006 has been obtained to facilitate completion of the remaining tasks and to allow the students to complete their theses.

Overall Issues and Concerns: None.

\section{PAPERS AND PRESENTATIONS}

1. P. Y. Park. M. U. Macdonald, D. D. Macdonald, "Application of the PDM (Point Defect Model) to the Oxidation of Zircaloy Fuel Cladding in Water-Cooled Nuclear Reactors", ICONE 12, V1, Page 605-613. (2004)

2. P. Y. Park. M. U. Macdonald, D. D. Macdonald, "Application of the PDM (Point Defect Model) to the Oxidation of Zircaloy Fuel Cladding in Water-Cooled 
Nuclear Reactors", ICONE, 12th International Conference on Nuclear Engineering, "Nuclear Energy - Powering the Future", Hyatt Regency Crystal City, Arlington, Virginia (Washington, D.C.),USA, April 25-29. (2004).

3. Yingzi Chen and Digby D. Macdonald, "Analysis of Transient Passive Film Growth in High Temperature Aqueous Solution", 206th Electrochemistry Society Annual Meeting, Honolulu, Hawaii, October 3-8. (2004).

4. Yingzi Chen and Digby D. Macdonald, "Analysis of Transient Passive Film Growth in High Temperature Aqueous Solution", Proc. Symp. Pits and Pores, The Electrochemical Society, Pennington, NJ, in press (2004).

5. Digby D. Macdonald, "Stress Corrosion Cracking in Reactor Coolant Circuits an Electrochemist's Viewpoint", International Water Conference, San Francisco, October 17-21, 2004.

6. Digby D. Macdonald, "Stress Corrosion Cracking in Reactor Coolant Circuits an Electrochemist's Viewpoint", Power Plant Chemistry, Vol. 12, page 731-747. (2004).

7. Yingzi Chen, Mirna Urquidi-Macdonald, Digby Macdonald, "The Electrochemistry of Zirconium in Aqueous Solutions at Elevated Temperatures and Pressures" Journal of Nuclear Materials. 348, 133-147. (2006).

8. Yingzi Chen, Mirna Urquidi-Macdonald, Digby Macdonald, "Transient Oxide Film Growth on Zirconium in High Temperature Aqueous Solution", paper for the Proceedings of 12th International Conference on Environmental Degradation of Materials in Nuclear Systems-Water Reactors, Salt Lake City, Utah, August 14-18. (2005). accepted.

9. Yingzi Chen, Mirna Urquidi-Macdonald, Digby Macdonald, "Transient Oxide Film Growth on Zirconium in High Temperature Aqueous Solution", submitted to the Metallurgical and Materials Transactions B. (2005).

10. Jiahe Ai, Yingzi Chen, Mirna Urquidi-Macdonald, Digby D. Macdonald, "Electrochemical Impedance Spectroscopic Study of Passive Zirconium in High Temperature Deaerated Aqueous Solutions", ECS Transactions of 209th Meeting of The Electrochemical Society, Denver, Colorado, May 7-11, (2006).

11. Jiahe Ai, Yingzi Chen, Mirna Urquidi-Macdonald, Digby D. Macdonald, "Electrochemical Impedance Spectroscopic Study of Passive Zirconium: Part 1. High Temperature, Deaerated Aqueous Solutions", Journal of the Electrochemical Society, 154(1), (2007), C43.

12. Jiahe Ai, Yingzi Chen, Mirna Urquidi-Macdonald, Digby D. Macdonald, "Electrochemical Impedance Spectroscopic Study of Zirconium: Part 2. High Temperature, Hydrogenated Aqueous Solutions", submitted to Journal of the Electrochemical Society. 154(1), (2007), C52. 
13. D. D. Macdonald, Y. Chen, J. Ai, M. Urquidi-Macdonald, “An Electrochemical Impedance Spectroscopic Definition of the Passive State on Zirconium in Simulated LWR Coolant Environments", 8th International Symposium on Advances in Electrochem. Sci. and Tech. (ISAEST-8) NIO, Goa, Nov.28-30, (2006)

14. J. Ai, Y. Chen, M. Urquidi-Macdonald, D. D. Macdonald, "Electrochemical impedance study of zirconium in high temperature hydrogenated aqueous solutions". 210th Meeting of Electrochemical Society, Cancun, Mexico, Oct.29Nov.2, (2006)

15. Jiahe Ai, Yingzi Chen, Mirna Urquidi-Macdonald, Digby D. Macdonald, "Stability of Passive Films on Zirconium in High Temperature De-aerated Aqueous Solutions", being modified, and will be submitted to a journal.

16. Jiahe Ai, JeongSeb Han, Digby D. Macdonald, "Theoretical Model for Hydrogeninduced Cracking in Aqueous Environments", being modified, and will be submitted to a journal.

\section{V.STUDENT DISSERTATIONS}

1. Pilyeon Park, "The Application of the PDM (Point Defect Model) to the Oxidation of Zircaloy Fuel Cell Cladding in Water-Cooled Nuclear Reactors", MS Thesis, Department of Engineering Science and Mechanics, Pennsylvania State University, University Park, PA, September 2004.

2. Yingzi Chen, "The Oxidation and Hydriding of Zirconium and Zircaloys in High Temperature Aqueous Solutions", PhD Dissertation, Department of Materials Science and Engineering, Pennsylvania State University, University Park, PA, February 2006.

\section{COST}

A. Performance:

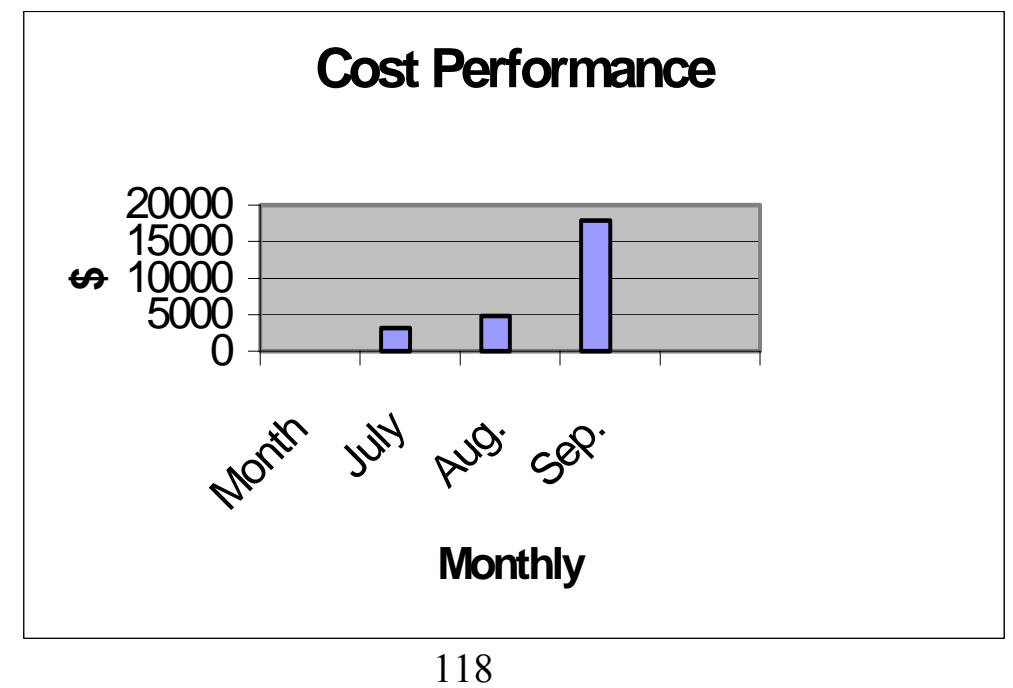

